\title{
A Multifaceted Approach to Improving Transgenic \\ Technology in Maize
}

A Dissertation
presented to
the Faculty of the Graduate School
at the University of Missouri
In Partial Fulfillment
of the Requirements for the Degree
Doctor of Philosophy
By
NATHANIEL GRAHAM
Dr. James Birchler, Dissertation Supervisor
July 2017


The undersigned, appointed by the dean of the Graduate School, have examined the dissertation entitled

\begin{abstract}
A Multifaceted Approach to Improving Transgenic Technology in Maize

Presented by Nathaniel Graham, a candidate for the degree of doctor of philosophy, and hereby certify that, in their opinion, it is worthy of acceptance.
\end{abstract}

Dr. James Birchler

Dr. Kathleen Newton

Dr. David Braun

Dr. Sherry Flint-Garcia 


\section{Acknowledgements}

I would like to acknowledge the strong support that has been given to me by the department of Biological Sciences at the University of Missouri, especially from my advisor Dr. James Birchler. Without his encouragement and guidance this work may not have existed. It has been an honor to have been mentored by someone with such scientific brilliance in the field while at the same time someone with the modesty he displays.

I would also like to acknowledge the support that has been granted to me by my loving friends and family. Without their encouragement, I would not have been able to get through the lows to make it to the highs. I would like to especially acknowledge the support that my parents have shown me over the years. While I know they don't quite understand the process of a doctorate, they have been completely supportive nonetheless. Lastly, I would like to thank the support that my loving wife Shelby has shown through this process. Being the spouse of a doctoral candidate is not easy, and can be extremely stressful. Shelby has shown non-ending support, despite the added stress to her own career and plans. I cannot express my gratitude enough. 


\section{Table of Contents}

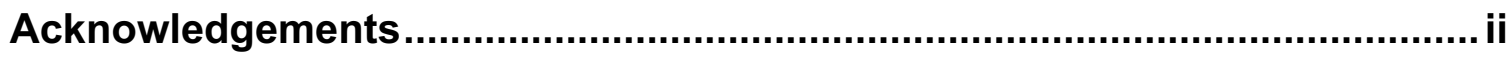

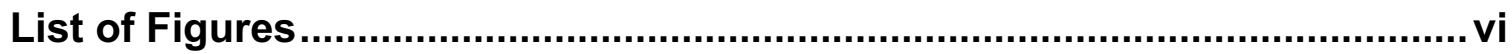

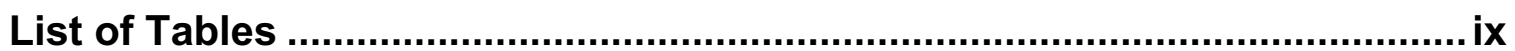

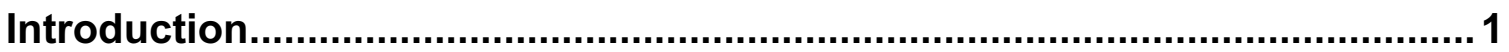

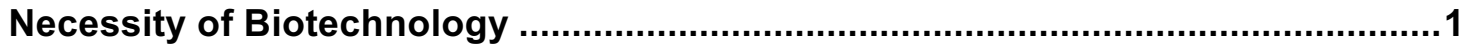

Why Is It Necessary to Improve Current Biotechnology Techniques in Maize? ...2

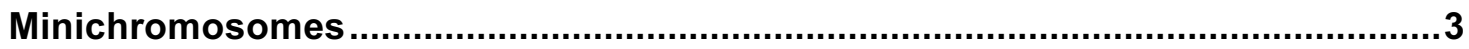

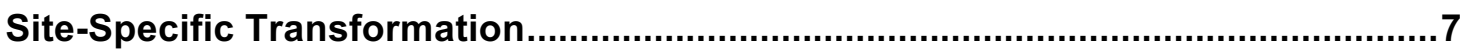

References

Chapter 1 An Improved Agrobacterium tumefaciens Transformation

Protocol for Zea mays Developed from a Meta-Analysis of Patented

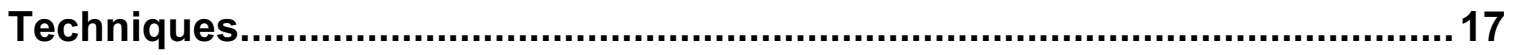

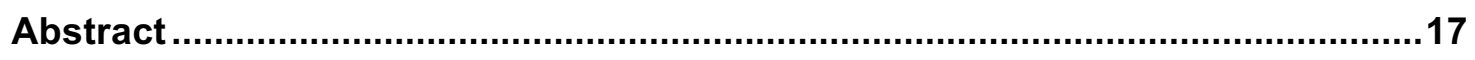

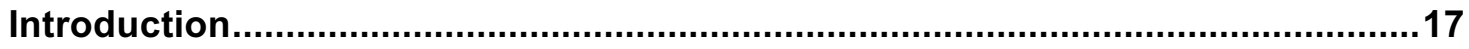

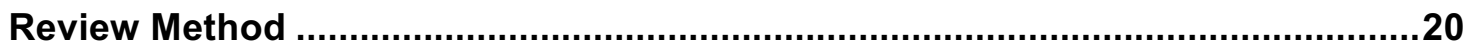

Patent Review Tables and Consensus Protocol .................................................21

Patent Review and Formation of Consensus Protocol.........................................36

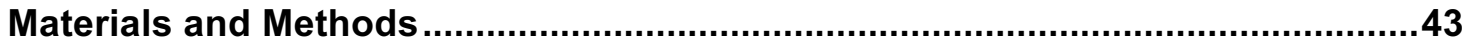

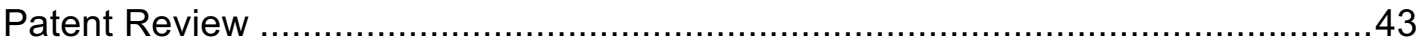

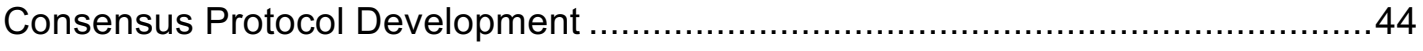

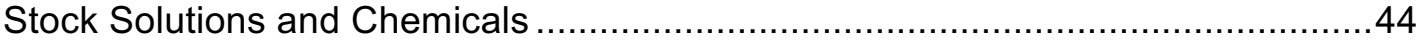

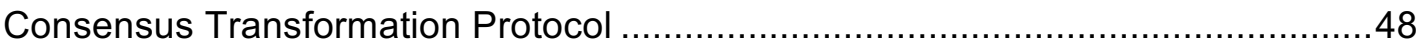




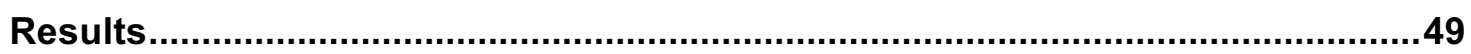

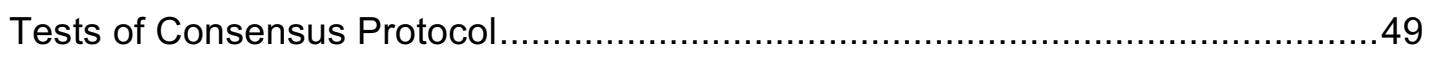

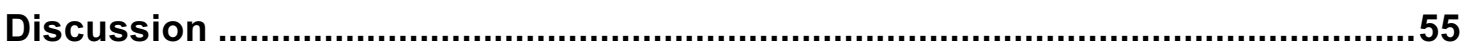

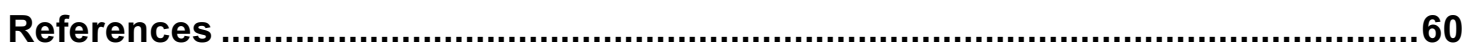

Chapter 2 Demonstration of $\Phi C 31$ Integrase and Excisionase Activity in Zea

mays

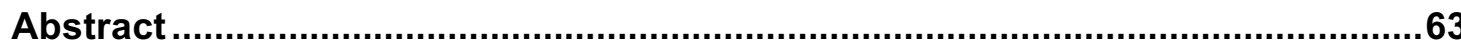

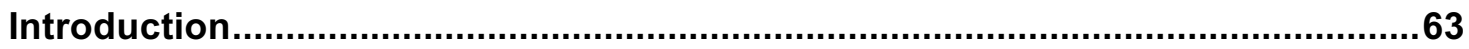

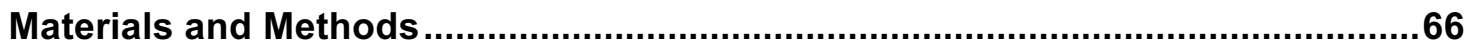

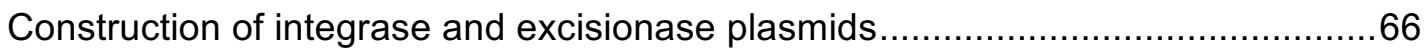

Maize Transformation of Integrase and Excisionase..........................................68

Construction of Integrase and Excisionase Reporter Constructs ...........................68

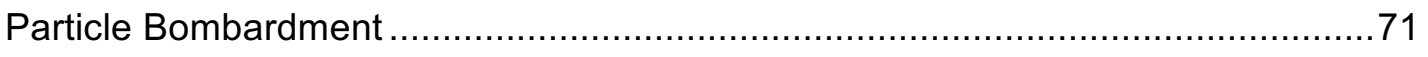

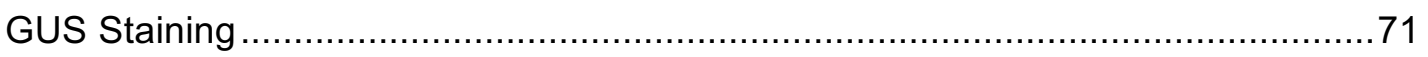

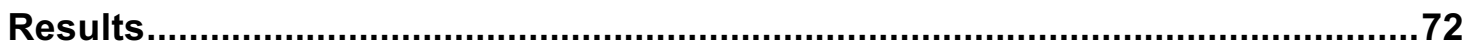

Transient Expression of Integrase and Excisionase ..........................................72

Activity of $\Phi C 31$ Integrase and Excisionase when Transformed into Maize ...........74

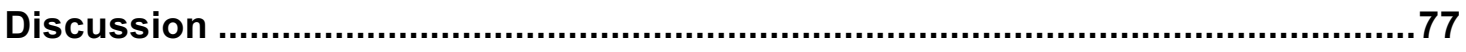

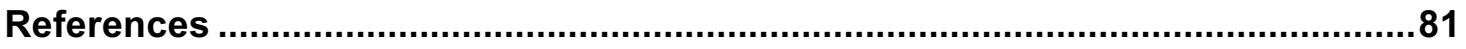

Chapter 3 Evidence for Site-Specific Transgene Integration Using ФC31

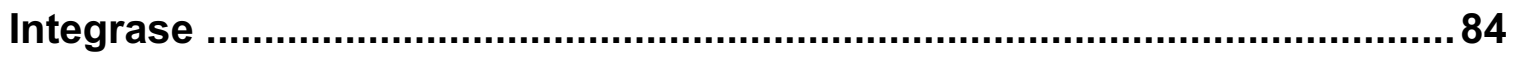

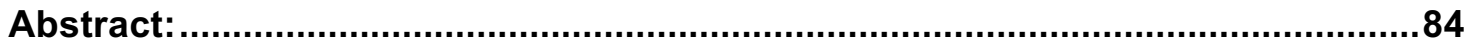

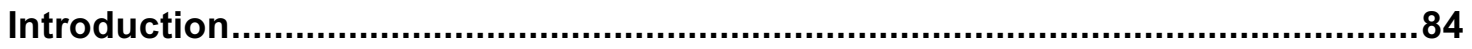

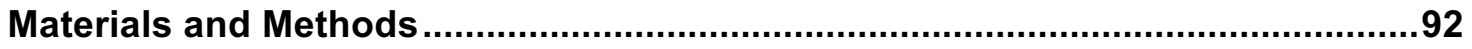




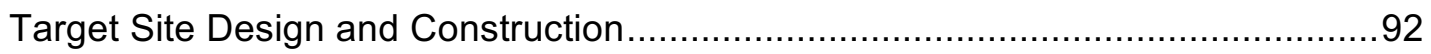

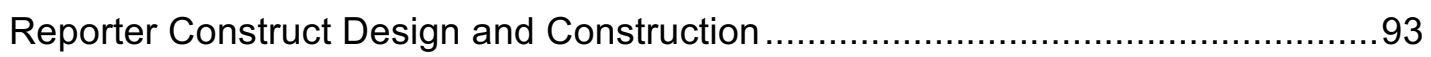

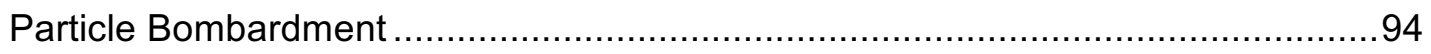

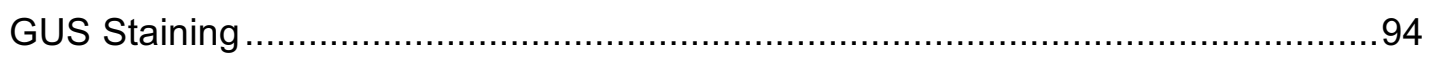

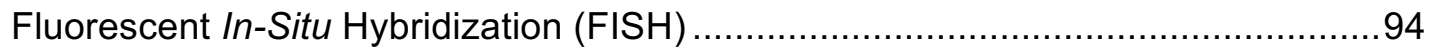

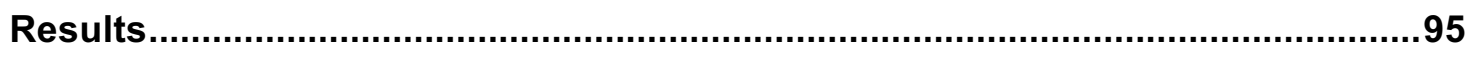

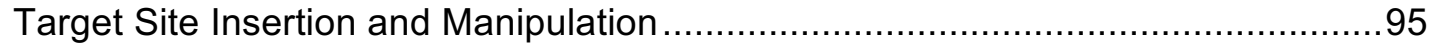

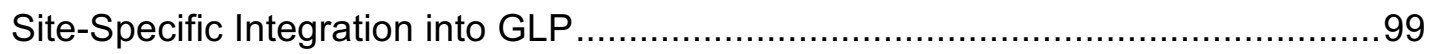

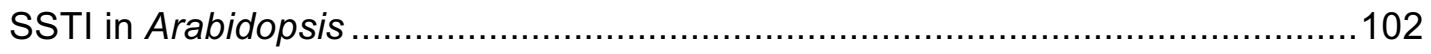

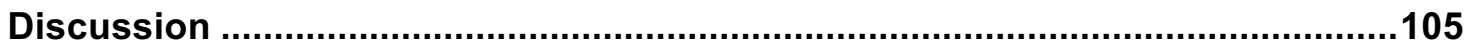

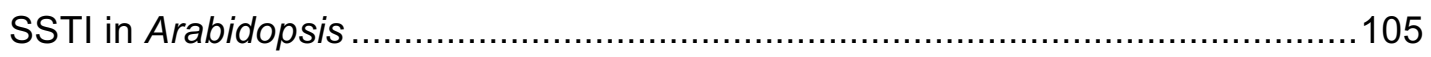

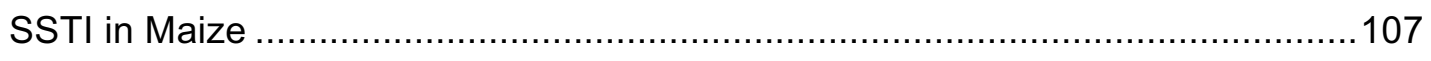

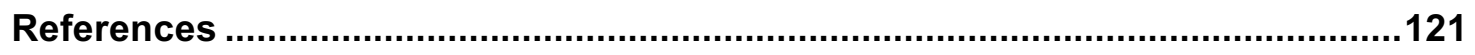

\section{Chapter 4 Production of Minichromosome Vectors via the Introduction of}

Telomere Sequences ............................................................................... 127

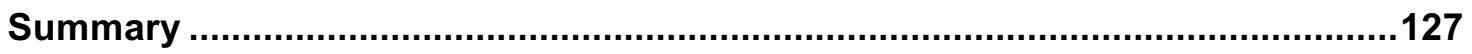

1. Introduction........................................................................................ 128

Protocol 1: Ligation of Telomere Sequences within Agrobacterium

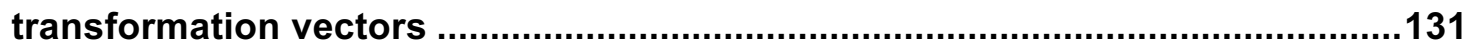

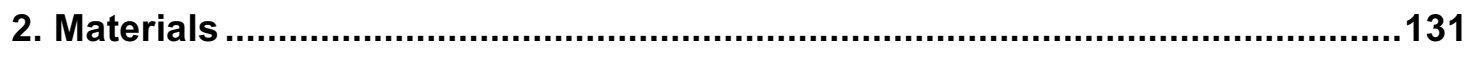

2.1 Materials for in-gel ligation into Agrobacterium competent vector ..................131

2.2 Equipment for in-gel ligation into Agrobacterium competent vector ............... 132

2.3 Recipes for in-gel ligation into Agrobacterium competent vector ..................133

2.4 Materials for preparation of telomere for particle bombardment...................133

2.5 Equipment for preparation of telomere for particle bombardment .................. 134 
3. Methods

3.1 Insertion of Telomere through In-Gel Ligation

3.1.1 Screening potential colonies for telomere size 140

3.2 Production of Telomere Fragments via Polymerase Chain Reaction 141

3.3 Transgene Delivery into Plants with Telomere Arrays. .143

4. Notes. 144

Acknowledgement 147

References 147

\section{Chapter 5 Producing a BiBAC Vector for the Production of}

Minichromosomes in Maize. 149

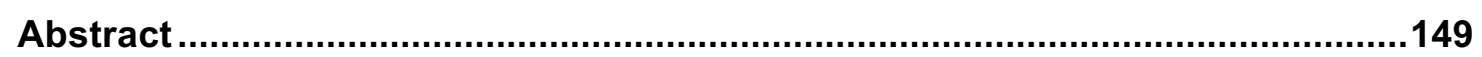

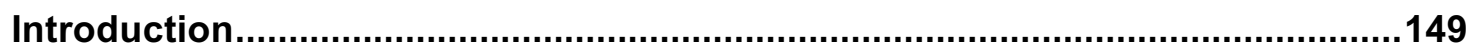

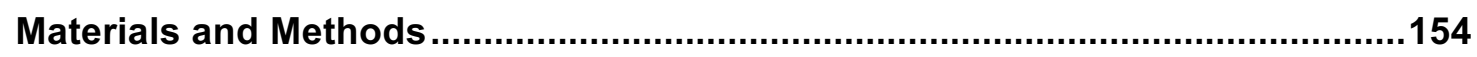

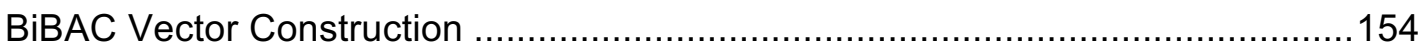

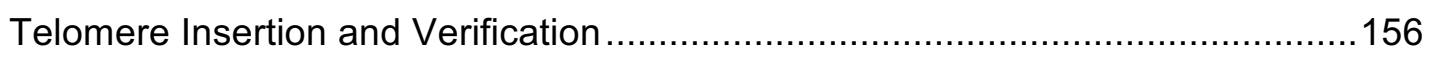

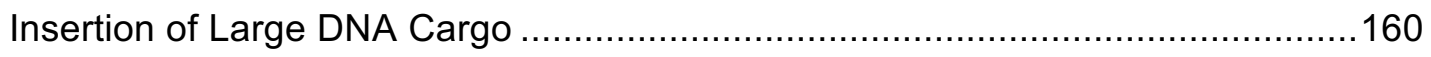

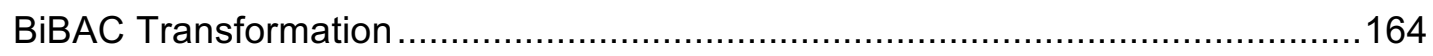

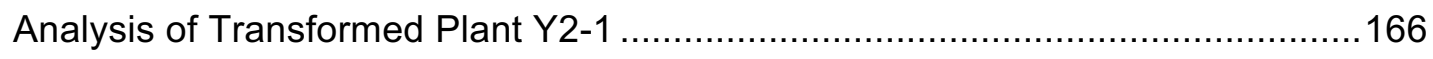

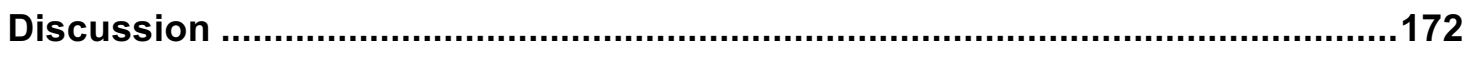

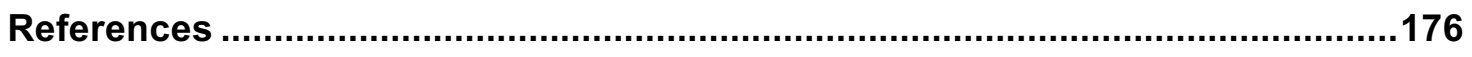

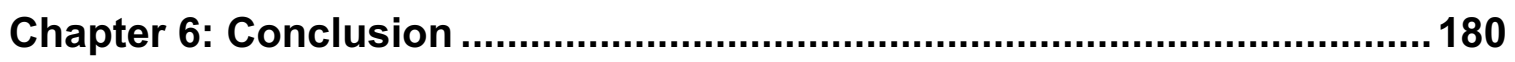

VITA

\section{List of Figures}


Figure 1 Callus Growth After 10 Days on Resting Media................................51

Figure 2 Callus Selection After 2 weeks of S2 Selection ...............................52

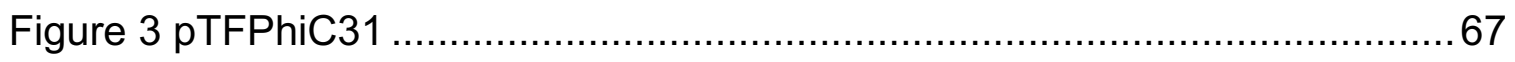

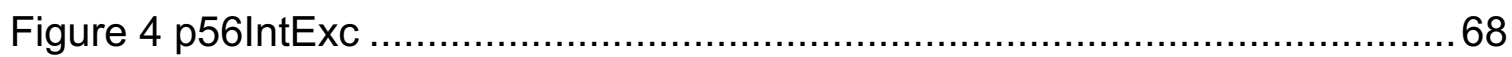

Figure 5 Reporter Strategy for Demonstrating Recombinase Activity ................69

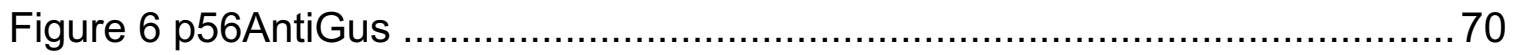

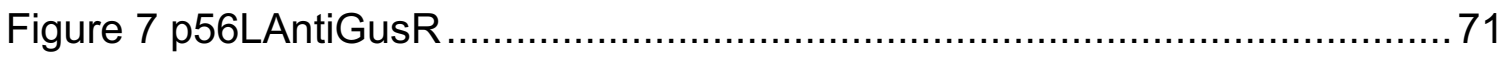

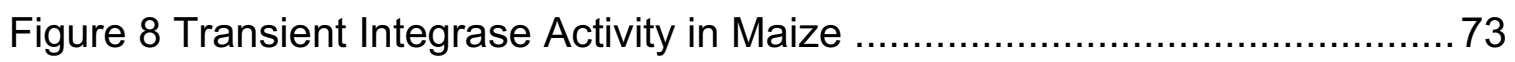

Figure 9 Transient Activity of Excisionase in Maize ..................................... 74

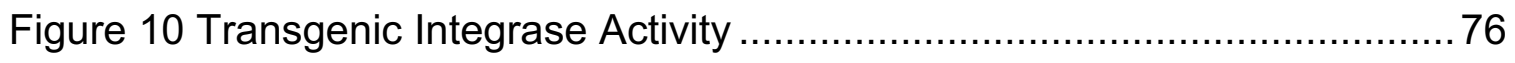

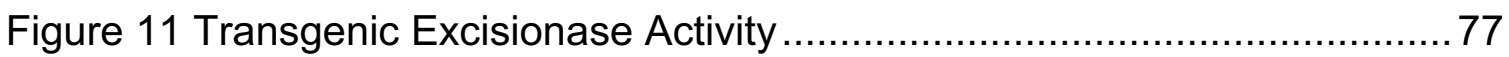

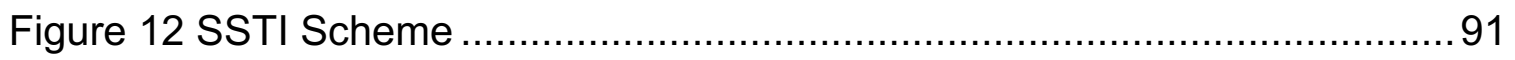

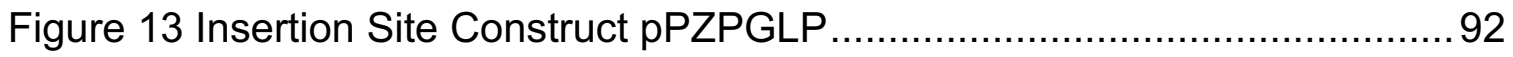

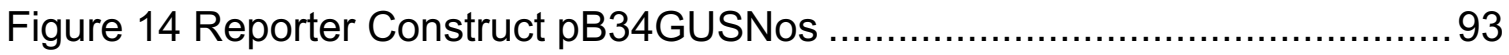

Figure 15 FISH Image of Homozygous GLP1 Plant....................................... 96

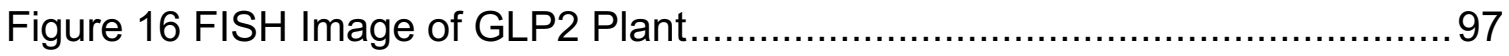

Figure 17 Representative Gel Image of GLP PCR ..................................... 98

Figure 18 Sanger Sequencing of Reduced GLP ..........................................99

Figure 19 Demonstration of SSTI in Maize with Reporter Plasmid .................. 100

Figure 20 Negative Control Embryo Showing GUS Signal ........................... 101

Figure 21 Hygromycin Integration Construct pPZPBHYGNos ....................... 102

Figure 22 Hygromycin Integration Construct with Lox Sites............................ 104

Figure 23 Example of Arabidopsis Dip Hygromycin Screen.......................... 105 
Figure 24 Insertion Construct for Glyphosate Selection .................................109

Figure 25 Recurrent SSTI Scheme for Maize …....................................... 115

Figure 26 Alternate Recurrent SSTI Strategy ............................................ 119

Figure 27 Example Digest of pWY82 .................................................... 136

Figure 28 Example Gel Used for Telomere Ligation. ....................................138

Figure 29 Telomere PCR Example Using 20 ul and 40 ul Reaction Volumes. .143

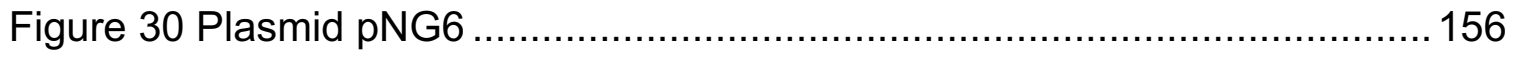

Figure 31 Digest pNG6 Colonies Containing Telomere .................................158

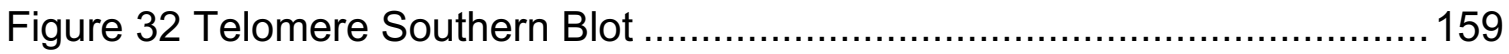

Figure 33 Pulsed Field Gel of Yeast BiBAC Colonies ................................... 160

Figure 34 Example IGV Alignment of BiBAC Sequencing ............................ 161

Figure 35 Digest of pNG6+T1.8 Yeast Colonies for Telomere Southern

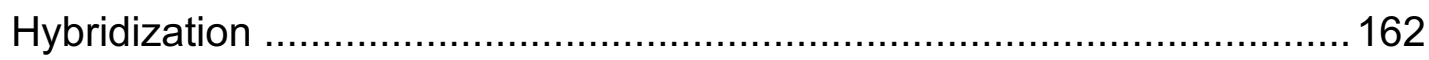

Figure 36 Southern Hybridization of pNG6+T1.8 Yeast Colonies ..................... 163

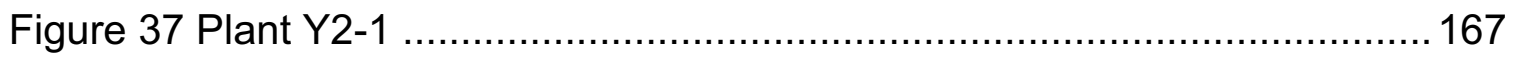

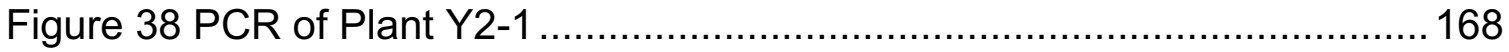

Figure 39 FISH Image of Plant Y2-1 ..................................................... 170

Figure 40 Single-Gene FISH of Plant Y2-1 ............................................ 171 


\section{List of Tables}

Table 1 Literature Sources for Protocol Review................................. 23

Table 2 Inoculation Media..................................................... 24

Table 2 Inoculation Media Continued........................................... 25

Table 3 CoCultivation Media................................................. 26

Table 3 CoCultivation Media Continued...................................... 27

Table 4 Resting Media..................................................... 28

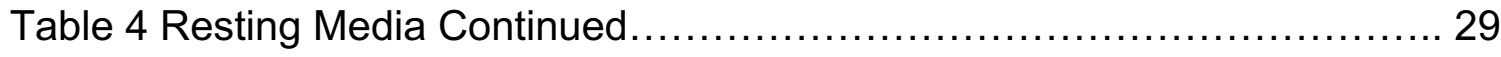

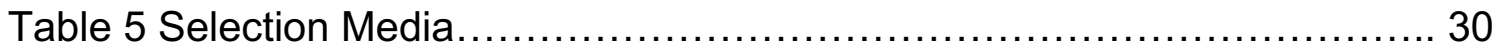

Table 5 Selection Media Continued.......................................... 31

Table 6 Shooting Media....................................................... 32

Table 6 Shooting Media Continued......................................... 33

Table 7 Rooting Media...................................................... 34

Table 7 Rooting Media Continued............................................. 35

Table 8 Chemicals Used in Transformation Protocol............................. 46

Table 9 Final Consensus Protocol........................................... 47

Table 10 Summary of Yeast BiBAC Data....................................... 164 


\section{Introduction}

\section{Necessity of Biotechnology}

From recent estimates, the world's population has reached 7 billion, and by 2050 the population is predicted to approach 10 billion (United Nations, Department of Economic and Social Affairs, 2015). This exponential growth is going to require an unprecedented increase in food production. To compensate for this growth, agricultural production will need to increase at a rate much higher than population growth, as many countries in the world will switch to a meatbased diet, which requires more agricultural input. The combination of increasing population, which will necessitate switching agricultural lands to urban construction to facilitate larger city centers, and a changing world diet, which will require larger land inputs, are at odds. The result is a requirement to produce $69 \%$ more calories by the year 2050 , on a much smaller land area. To address this issue, large amounts of resources have been devoted to breeding technology to improve plant vigor and output. The result is an increase in yield over 2.7 times the levels of 1948 in 2013 (Ball et al., 2017). However, despite these increases, the agricultural demands will be much greater in the coming years.

In the early 1990's the first products were introduced that were the result of biotechnology research efforts. These products, which combined plant biotechnology and hybrid breeding, allowed scientists to target specific trait improvements. Currently, over $90 \%$ of the acreage planted in the United States is 
transgenic (ISAAA, 2016), reflecting the acceptance of the technology by the agricultural industry. In order to maintain production requirements in the upcoming years, biotechnology will be a necessity.

\section{Why Is It Necessary to Improve Current Biotechnology}

\section{Techniques in Maize?}

Despite the acceptance of biotech products to the marketplace, and demand for future improvements, the technology itself has remained relatively unchanged. Most transformation based genetic modifications in plants are a result of Agrobacterium tumefaciens transformation and tissue culture. In Zea mays, which will be the focus of this work, this process has generally been the same since the early 1990 s. When including the time required to grow plants, the full procedure takes 10 months to 1.5 years to obtain T0 seed, with only $5-10 \%$ of the original embryos being transformed into full grown plants. As such, the technique itself is a strong limiting factor in the use of biotechnology in maize.

In addition to taking a large amount of time to complete the process, there are other issues with the transformation process in maize that need to be addressed to completely take advantage of the technology. The maize line generally used for transformation (Hi-II), is weak with low vigor. As a result, new

transformants are usually crossed onto more vigorous lines following the transformation process. However, due to linkage drag it is usually required to carry out repeated back crosses to completely remove the undesired $\mathrm{Hi}-\mathrm{Il}$ traits, and fully integrate the new transformed gene into the new line. 
Another issue with current transformation technology is the random nature of the integration process. While there have been studies suggesting that T-DNA integration preferentially inserts into transcriptionally active (Koncz et al., 1989) or regulatory regions (Chen et al., 2003), while other studies suggest that the system is indeed random (Kim and Gelvin, 2007). In either case, the process can yield transgene insertions in any chromosome, with a large range of positional effects possible. The end result being that multiple events of the same transgene can have different expression profiles. Additionally, there is a risk that the incoming transgene will insert inside of a gene, causing an insertional mutation.

\section{Minichromosomes}

Many of the problems with current biotechnology techniques in maize can be alleviated with the use of minichromosomes, small autonomous chromosomes that exist outside of the normal chromosome set. Minichromosomes contain all of the necessary components required to be maintained from generation to generation and can thus be utilized as vectors for plant transgenes. As minichromosomes are maintained outside of the normal genome, inserted transgenes are not susceptible to linkage drag when being transferred between lines. Additionally, there is no risk of insertional mutagenesis as there are no known genes present with which to interfere.

Minichromosomes are usually produced through the top-down method, utilizing an endogenous chromosome already present in the cell. One of the main reasons for using the top-down method is the epigenetic nature of the plant centromere. While centromere sequences are enough to confer activity in yeast, 
this is not the case in plants. This can be demonstrated by centromere inactivation on chromosomes containing two sets of centromeric DNA (Han et al., 2006), or similarly by activation of a new centromere where there was not previously centromeric DNA present (Nasuda et al., 2005). Additionally, attempts to form active centromeres by introducing centromere repeats into rice only resulted in stable integrations as opposed to newly active centromeres (Phan et al., 2007). The inability to form new centromeres simply by introducing centromere repeats thus necessitates that a minichromosome utilize an already active centromere in the cell.

Minichromosomes in plants are produced by telomere-mediated chromosomal truncation. In this process, a transgene is inserted containing a telomere sequence array. In the case of particle bombardment, the telomere sequences can be included as part of the transgene cassette, or cobombarded as a separate sequence (Gaeta et al., 2013). For Agrobacterium transformation, the telomere sequences are introduced within the right border of the T-DNA (Yu et al., 2006). For both techniques, upon insertion into the genome the transgene can either be fully integrated into the genome, or the end-joining machinery will recognize the telomere sequences and fail to repair the DNA break at this site. In this case, anything distal to the insertion site will be lost, forming a truncation event that generates a minichromosome. Telomere-mediated chromosomal truncation to produce minichromosomes was first demonstrated in plants in maize (Yu et al., 2006), and has since been shown to work in Arabidopsis 
(Nelson et al., 2011; Teo et al., 2011), barley (Kapusi et al., 2012), and rice (Xu et al., 2012).

In maize, the B chromosome is the endogenous chromosome typically targeted for minichromosome production. The B chromosome is a supernumerary, telocentric chromosome that is not known to pair with any of the A chromosomes and is maintained due to an accumulation mechanism (Jones and Houben, 2003). The genes responsible for the accumulation mechanism are the only known genes present on the chromosome. While the genes have not been identified, one site required for nondisjunction is known to be located on the distal tip of the long-arm (Ward, 1973). The accumulation mechanism works in trans against the $\mathrm{B}$ chromosome centromere, causing it to non-disjoin at the second pollen mitosis (Roman, 1947). The sperm containing the B chromosomes also exhibit preferential fertilization of the egg, rather than the polar nuclei, as part of the accumulation mechanism (Roman, 1948).

While the accumulation mechanism has allowed the B chromosome to be maintained despite not containing any necessary genes, it makes a normal B chromosome a poor target for transgene insertion. Manipulation of transgene dosage would be difficult on a B chromosome, as transmission of the transgene would not follow normal Mendelian ratios. However, using the B chromosome as a target for minichromosome production makes it an ideal vector. Chromosomal truncation of the long arm will inactivate the accumulation mechanism, leaving the transgene outside of the genome on a chromosome that behaves more predictably. However, reintroducing the trans-acting accumulation mechanism 
through a full-sized B chromosome will allow transgene dosage manipulation if desired (Masonbrink and Birchler, 2012). The stability of the minichromosome will ultimately depend on how large it is. Studies have shown that smaller minichromosomes will lag during meiosis, effecting transmission rates; however, larger minichromosomes will behave similarly to full-sized B chromosomes (Masonbrink et al., 2012).

Despite the utility of the minichromosome system, there are still aspects that need improvement. First, whether using Agrobacterium transformation or particle bombardment, the system relies on the random transformation process. As a result, many of the transformation events will insert the transgene at less than optimal locations. Additionally, truncation relies on the cell machinery recognizing the incoming telomere sequences. Thus, even if the transgene is inserted onto a B chromosome, it may not cause a truncation event. Additionally, the transgene must insert in the correct orientation so that it is not lost. If the telomere sequences are oriented towards the centromere of the truncated chromosome, the transgene will cause an acentric fragment and be lost. All of these factors, in addition to the low transformation efficiency in maize, lead to a low frequency of minichromosome recovery.

The minichromosome system is also hindered by the amount of genetic cargo that can be transferred upon transformation. Standard transformation vectors are limited to about $15-20 \mathrm{~kb}$ in size, which restricts the amount of genetic cargo that can be integrated. Additionally, in the case of Agrobacterium transformation, the inclusion of a secondary origin of replication and telomere 
sequences restricts the amount of cargo even further. To increase the amount of genetic cargo that a plasmid can carry, the $\mathrm{F}$ origin of replication can be included, creating a bacterial artificial chromosome (BAC) vector. When this origin is included in a Agrobacterium-competent binary vector, a binary bacterial artificial chromosome (BiBAC) vector is produced (Hamilton, 1997). These vectors have been shown to transfer extremely large genetic cargos (over $150 \mathrm{~kb}$ ) in plant systems with Agrobacterium transformation methods (Frary and Hamilton, 2001). Including telomere sequences in a BiBAC vector should allow the vector to produce a minichromosome while also introducing a large genetic cargo. However, initial experiments were unsuccessful (Vega et al., 2008a). While the vector was capable of transferring at least $30 \mathrm{~kb}$ of genetic cargo, there were no truncation events found. This may be due to the fact that the telomere repeat array was included at the left border of the plasmid. Other studies producing minichromosomes have placed the telomere array at the right border, suggesting that the right border is more open to recognition by cell machinery for chromosome capping. Additionally, plants were not screened until the T1 stage, so it is possible that A chromosomes were truncated, but selected against during meiosis due to a loss of genetic material.

\section{Site-Specific Transformation}

As previously discussed, reliance on a random transformation system contributes to low transformation efficiency and minichromosome generation in 
maize. One suggested remedy for this problem is using site-specific recombination systems. Site-specific recombination systems have been utilized in plants for decades for the removal of selection genes (Dale and Ow, 1991), with the most commonly used recombinase system being the Cre/lox system. Recombinases target and recombine specific DNA sequences, and depending on orientation can result in excision or inversion of any DNA between recognition sites. If a recognition site is located within the genome already, a recombinase can integrate plasmid DNA that contains a corresponding recognition site. As a result, recombination sites can be inserted into the genome and new DNA sequences targeted for insertion at that site, removing the randomness from the transformation scheme.

Site-specific integration has been in use in plants, albeit rarely, since the early 1990's (Albert et al., 1995). Until recently, this approach has been focused on the Cre/lox system. This system, while useful, is limited due to its reversibility. After recombination, lox sites are left fully intact, meaning that if used for integration the new DNA sequence will be flanked by lox sites, which can recombine again, excising the construct. In fact, the excision reaction is kinetically favored because of the proximity of the lox sites. This can be overcome by utilizing mutant lox sites that result in a non-functional recombination site after the reaction (Albert et al., 1995).

Recently, more recombinases have been tested in plants, allowing for greater flexibility with site-specific transformation (reviewed in Graham et al. 2015). By combining recombinases, a system can be developed that allows 
continued addition of transgenes to a single locus. One of the first proposed systems combined the Cre/lox, FLP/FRT, and R/RS recombinases together such that a transgene could be integrated, non-desired sequences removed, and then another transgene inserted into the newly integrated DNA (Srivastava and Ow, 2004). While this system does allow for subsequent additions, all the recombination reactions are reversible, leading to inefficiency.

When designing a system to be used in maize, it is imperative that the system be based around a unidirectional recombinase as the transformation process is already extremely inefficient. The system chosen for the work in this dissertation is based on the recombinase $\Phi C 31$ integrase, a large serine recombinase from the Streptomyces phage ФC31. ФC31 integrase recombines attP and attB sites in a unidirectional manner, resulting in att $L$ and attR sites (Groth et al., 2000). The reaction can only be reversed in the presence of an additional protein, recombination directionality factor (Khaleel et al., 2011), also known as excisionase. An attP or attB site introduced into the genome can act as an insertion site for further transgenes to be targeted. When combined with the Cre/lox system already known to be functional in maize (Gaeta et al., 2013), a system can be devised that allows for successive addition to a genomic locus, while also repeatedly utilizing bialaphos for selection.

Utilizing $\Phi C 31$ integrase for transformation has the potential to improve maize transformation efficiency. Provided the plant is already expressing $\Phi C 31$ integrase, any circular transgene has the potential of being recognized and inserted into the genome. Additionally, as the pre-inserted site has already been 
utilized for selection once, it is already known to be transcriptionally active. By utilizing particle bombardment, circular plasmids can be introduced to plants that contain an attachment site target and are expressing $\Phi C 31$ integrase. Previous studies have shown that the amount of transient expression from particle bombardment is quite high (Oard et al., 1990), with multiple signals detected on each embryo subjected to treatment. In contrast, only $5-10 \%$ of embryos subjected to transformation and selection are generally recovered with standard techniques. By utilizing $\Phi C 31$ integrase in the transformation process, there is a potential for a much higher transformation rate.

Finally, combining site-specific transformation with minichromosome technology will allow for an improved platform for biotechnology. Minichromosomes originally produced containing recombination sites can be easily targeted with future transgenes. Instead of complex crossing schemes, additional transgenes could be combined directly onto the minichromosome locus. A well characterized minichromosome containing an attachment site should allow for consistent expression of new transgenes, allowing researchers to reliably compare inserted transgenes. Additionally, as minichromosomes contain no known genes, combining multiple minichromosomes together to combine traits should not result in any endogenous gene dosage effects.

The following chapters represent the progress made towards improving transgenic maize technology. In chapter one, a meta-analysis is performed to examine the various improvements to media and transformation techniques that have been made in the industry sector. After reviewing patents filed by the major 
companies, a consensus protocol was formed that contained the most common improvements to the technique. In chapter two, the recombinase $\Phi C 31$ integrase and the associated protein $\Phi C 31$ excisionase are tested for functionality in maize embryos. Using the GUS reporter, the recombinases are shown to be functional both when integrated into the genome and when introduced for transient expression. Chapter three examines whether the recombinase $\Phi C 31$ integrase can be utilized for site-specific transgene integration. A target site is first placed into the genome that contains a removable selectable marker, which is then removed via the Cre recombinase system. After recombination, the $\Phi \mathrm{C} 31$ recombination site attP is left behind a ubiquitin promoter, which has already been demonstrated to be functional. This target site is then targeted with an integration construct containing GUS that lacks a promoter. When introduced with $\Phi C 31$ integrase, the GUS reporter was activated, suggesting that the reporter had been integrated into the target site. In chapter four, a protocol which has been published in "Methods in Molecular Biology" (Graham et al., 2016), techniques are demonstrated for the construction of a truncation vector for the production a minichromosome. The novel portions of the protocol pertain to the techniques for cloning and detecting telomere sequences. Due to the complexity of the telomere repeats, traditional cloning methods were found to be ineffective. To overcome these difficulties, telomere sequences are excised and ligated while still inside of an agarose gel. Additionally, as the presence of telomere repeats inhibit the activity of DNA polymerase, screening for positive insertions cannot be performed with PCR screening. To alleviate this issue, potential colonies are 
screened via colony PCR and Southern hybridization. Chapter five examines the use of binary bacterial artificial chromosome (BIBAC) vectors to produce minichromosomes. BIBAC vectors can allow hundreds of kilobases to be maintained in a single vector, which could allow for multiple transgenes to be inserted during minichromosome production. To test the feasibility of the system, a BIBAC vector was created containing telomere sequences and yeast DNA as a filler. This vector was used for maize transformation, but only a single regenerated plant was produced. While the vector was demonstrated as having the capacity to transfer large DNA cargos, more work is required to determine if it is capable of producing a minichromosome.

\section{References}

Albert, H., Dale, E., Lee, E., and Ow, D. (1995). Site-specific integration of DNA into wild-type and mutant lox sites placed in the plant genome. The Plant Journal : For Cell and Molecular Biology 7, 649-659.

Ball, E., Wang, S.L., and Nehring, R. (2017). Summary of Recent Findings.

Chen, S., Jin, W., Wang, M., Zhang, F., Zhou, J., Jia, Q., Wu, Y., Liu, F., and Wu, P. (2003). Distribution and characterization of over 1000 T-DNA tags in rice genome. Plant Journal 36, 105-113.

Dale, E., and Ow, D. (1991). Gene transfer with subsequent removal of the selection gene from the host genome. Proceedings of the National Academy of Sciences of the United States of America 88, 10558-10562.

Frary, A., and Hamilton, C.M. (2001). Efficiency and stability of high molecular 
weight DNA transformation: an analysis in tomato. Transgenic Research $10,121-132$.

Gaeta, R.T., Masonbrink, R.E., Zhao, C., Sanyal, A., Krishnaswamy, L., and Birchler, J.A. (2013). In vivo modification of a maize engineered minichromosome. Chromosoma 122, 221-232.

Graham, N., Swyers, N., Cody, J., McCaw, M., Zhao, C., and Birchler, J.A. (2016). Production of Engineered Minichromosome Vectors via the Introduction of Telomere Sequences. In Chromosome and Genomic Engineering in Plants: Methods and Protocols, M. Murata, ed. (New York, NY: Springer New York), pp. 1-13.

Graham, N.D., Cody, J.P., Swyers, N.C., McCaw, M.E., Zhao, C., and Birchler, J.A. (2015). Engineered Minichromosomes in Plants. In International Review of Cell and Molecular Biology, (Elsevier Ltd), pp. 63-119.

Groth, A.C., Olivares, E.C., Thyagarajan, B., and Calos, M.P. (2000). A phage integrase directs efficient site-specific integration in human cells. Proceedings of the National Academy of Sciences 97, 5995-6000. Hamilton, C.M. (1997). A binary-BAC system for plant transformation with highmolecular-weight DNA. Gene 200, 107-116.

Han, F., Lamb, J.C., and Birchler, J.A. (2006). High frequency of centromere inactivation resulting in stable dicentric chromosomes of maize. Proceedings of the National Academy of Sciences 103, 3238-3243. ISAAA (2016). Global Status of Commercialized Biotech/GM Crops: 2016 (Ithaca, NY). 
Jones, N., and Houben, A. (2003). B chromosomes in plants: escapees from the A chromosome genome? Trends in Plant Science 8, 417-423.

Kapusi, E., Ma, L., Teo, C.H., Hensel, G., Himmelbach, A., Schubert, I., Mette, M.F., Kumlehn, J., and Houben, A. (2012). Telomere-mediated truncation of barley chromosomes. Chromosoma 121, 181-190.

Khaleel, T., Younger, E., McEwan, A.R., Varghese, A.S., and Smith, M.C.M. (2011). A phage protein that binds PhiC31 integrase to switch its directionality. Molecular Microbiology 80, 1450-1463.

Kim, S.-I., and Gelvin, S.B. (2007). Genome-wide analysis of Agrobacterium TDNA integration sites in the Arabidopsis genome generated under nonselective conditions. The Plant Journal 51, 779-791.

Koncz, C., Martini, N., Mayerhofer, R., Koncz-Kalman, Z., Korber, H., Redei, G.P., and Schell, J. (1989). High-frequency T-DNA-mediated gene tagging in plants. Proceedings of the National Academy of Sciences 86, 84678471.

Masonbrink, R.E., and Birchler, J.A. (2012). Accumulation of Multiple Copies of Maize Minichromosomes. Cytogenetic and Genome Research 137, 5059.

Masonbrink, R.E., Gaeta, R.T., and Birchler, J.A. (2012). Multiple maize minichromosomes in meiosis. Chromosome Research 20, 395-402.

Nasuda, S., Hudakova, S., Schubert, I., Houben, A., and Endo, T.R. (2005). Stable barley chromosomes without centromeric repeats. Proceedings of the National Academy of Sciences 102, 9842-9847. 
Nelson, A.D., Lamb, J.C., Kobrossly, P.S., and Shippen, D.E. (2011). Parameters Affecting Telomere-Mediated Chromosomal Truncation in Arabidopsis. The Plant Cell 23, 2263-2272.

Oard, J.H., Paige, D.F., Simmonds, J.A., and Gradziel, T.M. (1990). Transient Gene Expression in Maize, Rice, and Wheat Cells Using an Airgun Apparatus. Plant Physiology 92, 334-339.

Phan, B.H., Jin, W., Topp, C.N., Zhong, C.X., Jiang, J., Dawe, R.K., and Parrott, W. a (2007). Transformation of rice with long DNA-segments consisting of random genomic DNA or centromere-specific DNA. Transgenic Research $16,341-351$.

Roman, H. (1947). Mitotic Nondisjunction in the Case of Interchanges Involving the B-Type Chromosome in Maize. Genetics 32, 391-409.

Roman, H. (1948). Directed Fertilization in Maize. Proceedings of the National Academy of Sciences 34, 36-42.

Srivastava, V., and Ow, D.W. (2004). Marker-free site-specific gene integration in plants. Trends in Biotechnology 22, 627-629.

Teo, C.H., Ma, L., Kapusi, E., Hensel, G., Kumlehn, J., Schubert, I., Houben, A., and Mette, M.F. (2011). Induction of telomere-mediated chromosomal truncation and stability of truncated chromosomes in Arabidopsis thaliana. The Plant Journal 68, 28-39.

United Nations, Department of Economic and Social Affairs, P.D. (2015). World Population Prospects 2015 - Data Booklet (ST/ESA/SER.A/377).

Vega, J.M., Yu, W., Han, F., Kato, A., Peters, E.M., Zhang, Z.J., and Birchler, J. 
a. (2008). Agrobacterium-mediated transformation of maize (Zea mays) with Cre-lox site specific recombination cassettes in BIBAC vectors. Plant Molecular Biology 66, 587-598.

Ward, E.J. (1973). Nondisjunction: localization of the controlling site in the maize B chromosome. Genetics 73, 387-391.

Xu, C., Cheng, Z., and Yu, W. (2012). Construction of rice mini-chromosomes by telomere-mediated chromosomal truncation. The Plant Journal 70, 10701079.

Yu, W., Lamb, J.C., Han, F., and Birchler, J. a (2006). Telomere-mediated chromosomal truncation in maize. Proceedings of the National Academy of Sciences 103, 17331-17336. 


\title{
Chapter 1 An Improved Agrobacterium tumefaciens \\ Transformation Protocol for Zea mays Developed from a Meta-Analysis of Patented Techniques
}

\begin{abstract}
The process of maize transformation remains largely cost prohibitive and time intensive. As a result, most transgenic production in academic labs is performed in transgenic centers specialized in the technique, which has remained largely unchanged since it's development. While not often published in academic journals, industry labs have focused on improvement of these techniques, as evident by the large number of patent applications on the subject. Here, a meta-analysis of a representative sample of these patents is performed and a consensus protocol formed that improves upon the current academic techniques.
\end{abstract}

\section{Introduction}

Over $92 \%$ of the 38 million hectares of maize fields planted in 2016 were transgenic (ISAAA, 2016). In the academic setting, production of transgenic maize lines remains an invaluable tool for research; however, few improvements have been made to the technique since it was first published in 1996 (Ishida et al., 1996). As a result, the technique remains costly and time intensive. Indeed, 
most researchers in the US rely on the few transgenic centers around the country specializing in the technique.

The two main transgenic facilities in the US are based at lowa State University and the University of Missouri, and rely on essentially the same protocol for transformation. The maize line Hi-Il is used as the source of embryos as it has been found to produce a high frequency of type II callus (Armstrong et al., 1991). The two variants ( $\mathrm{A}$ and $\mathrm{B}$ ) of maize line $\mathrm{Hi}-\mathrm{Il}$ are crossed and embryos harvested once they have reached $\sim 1.8-2 \mathrm{~mm}$ in length. Embryos are harvested, washed, and then briefly incubated in an Agrobacterium tumefaciens suspension. The infected embryos are then incubated on cocultivation media to allow the Agrobacterium to grow and transform embryos, before being switched to resting media containing antibiotics to remove the Agrobacterium cells. Embryos, which at this point have begun to differentiate into callus, are then moved onto growth medium containing a selection agent, typically bialaphos. The callus is then switched to new selection plates every few weeks until it is fast growing and embryoids can be seen under a microscope; the selection process typically takes a couple of months. Callus is then moved to shooting medium until well grown shoots have formed, and then moved to rooting medium. Once a plantlet with a well-formed root structure has grown, it is moved into soil in a growth chamber before being moved to the greenhouse. The entire process, from embryo collection until T0 seed can be selected, can take anywhere from 9 months to well over a year. Additionally, there is reasonable skill required throughout the process as well as a requirement for excellent sterile technique. 
As a result, the process of obtaining transgenic maize lines can be extremely cost prohibitive.

While most transformation in the academic sector is done through transformation facilities, this is often not the case in industry. Most agriculture companies producing transgenic products perform the transformation process inhouse, utilizing their own protocols. The larger companies, possessing massive research budgets, have a larger capacity to perform the experiments necessary to test the various components of the protocol. While these companies do not regularly publish these tests in academic journals, and indeed consider them confidential business information, the information can be found in patent applications. These applications are not peer-reviewed, and the claims within should not be taken as fact; however, a consensus amongst a large number of applications can be analyzed. Patent applications are filed by companies to protect research from being used by competitors, or to prevent competitors from patenting the techniques themselves and preventing a product from going to market. Additionally, companies often submit patent applications despite there being a low possibility of approval. The application can be referred to if a competitor attempts to patent the same technique as proof of prior art. The result is many applications being filed to prevent a rival company from patenting a technique used in the production of a product to be released. As millions of dollars are invested in each product that is taken to market, the desire to protect the research dollars invested is high. Despite the knowledge that companies are most likely investing a large amount of research dollars into the transformation 
process, and that the protocols used will be protected from competitors in patent applications, no reviews of transformation patent applications exist in the literature.

It is important to consider the best strategy to perform a patent review. An exhaustive review would not only be overly time intensive, due to the large number of applications filed, but also extremely difficult to perform due to the structure of patent applications. Most applications pertaining to transformation are not named as such, and information related to the technique is often contained within applications that would not originally be thought to be on the subject. Additionally, as patents do not pass through the peer-review process, any claims made should only be considered if made in multiple applications. Lastly, as patents are often filed in order to place company ideas within the literature, only claims made within the examples sections should be considered for review. This section presumably contains claims that were tested by the applicant.

\section{Review Method}

For the purposes of this review, patents were found using the Google Patents (patents.google.com) search tool. Relevant patents (table 1) were located and the transformation techniques recorded in tables 2-7. Documents were used that were patent filings or granted patents. In some cases, the filing was used in place of the granted patent as more information about the various techniques was given. Additionally, the few peer-reviewed protocols published from industry sources were also collected. For the most part, patents were 
considered that used the same type of process already used in academic transformation facilities, ie solid media on plates instead of liquid media in an automated facility. A representative sample of applications from the major companies were analyzed, with care made to locate applications from different time periods to see if changes were maintained over time. The various components were then compared across all the applications and a consensus protocol formed. To form the consensus protocol the various components were considered individually, with a stronger weight given to applications filed in a large number of countries as these applications reflect a larger investment from the company. This larger investment was thought to reflect a larger importance to the company as a whole and may indicate the technique included as actually being used to bring a product to market. The consensus protocol was then tested in the lab to examine whether there was an improvement over the standard protocol.

\section{Patent Review Tables and Consensus Protocol}

\begin{tabular}{|c|c|c|c|c|}
\hline Company & $\begin{array}{l}\text { Patent Number / } \\
\text { DOI }\end{array}$ & $\begin{array}{l}\text { Date Filed / } \\
\text { Published }\end{array}$ & $\begin{array}{l}\text { International } \\
\text { Filings }\end{array}$ & Reference \\
\hline Monsanto & $\begin{array}{l}2004 / 02 \\
44075\end{array}$ & $6 / 1 / 2004$ & 1 & $\begin{array}{l}\text { (Cai et } \\
\text { al., 2004) }\end{array}$ \\
\hline Monsanto & $\begin{array}{l}858103 \\
5\end{array}$ & $8 / 31 / 2007$ & 10 & $\begin{array}{l}\text { (Rout, } \\
\text { 2013) }\end{array}$ \\
\hline Monsanto & $\begin{array}{l}875960 \\
9\end{array}$ & $3 / 22 / 2010$ & 1 & $\begin{array}{l}\text { (Cai et } \\
\text { al., 2014) }\end{array}$ \\
\hline
\end{tabular}




\begin{tabular}{|c|c|c|c|c|}
\hline Monsanto & $\begin{array}{l}2013 / 02 \\
39253\end{array}$ & $3 / 8 / 2013$ & 10 & $\begin{array}{r}\text { (Rout } \\
\text { et al., 2013) }\end{array}$ \\
\hline Monsanto & $\begin{array}{l}896232 \\
6\end{array}$ & $7 / 18 / 2013$ & 10 & $\begin{array}{r}\text { (Akula } \\
\text { et al., 2015) }\end{array}$ \\
\hline Pioneer & $\begin{array}{l}814348 \\
4\end{array}$ & $1 / 21 / 2009$ & 4 & $\begin{array}{l}\text { (Mehlo } \\
\text { and Zhao, } \\
\text { 2012) }\end{array}$ \\
\hline Pioneer & $\begin{array}{l}840493 \\
0\end{array}$ & $1 / 26 / 2010$ & 1 & $\begin{array}{l}\text { (Wu et } \\
\text { al., 2013) }\end{array}$ \\
\hline Pioneer & $\begin{array}{l}2011 / 01 \\
67516\end{array}$ & $12 / 30 / 2010$ & 7 & $\begin{array}{l}\text { (Gordo } \\
\text { n-Kamm et al., } \\
2011)\end{array}$ \\
\hline Pioneer & $\begin{array}{l}2014 / 01 \\
73775\end{array}$ & $3 / 13 / 2013$ & 14 & $\begin{array}{r}\text { (Cho } \\
\text { et al., 2014) }\end{array}$ \\
\hline Pioneer & $\begin{array}{r}10.1105 \\
/ \text { tpc.16.00124 }\end{array}$ & $9 / 6 / 2016$ & $\mathrm{~N} / \mathrm{A}$ & $\begin{array}{r}\text { (Lowe } \\
\text { et al., 2016) }\end{array}$ \\
\hline $\begin{array}{c}\text { Japan } \\
\text { Tobacco Company }\end{array}$ & $\begin{array}{r}10.1038 \\
\text { /nbt0696-745 }\end{array}$ & $2 / 28 / 96$ & $\mathrm{~N} / \mathrm{A}$ & $\begin{array}{l}\text { (Ishida } \\
\text { et al., 1996) }\end{array}$ \\
\hline $\begin{array}{c}\text { Japan } \\
\text { Tobacco Company }\end{array}$ & $\begin{array}{l}\text { 2007/01 } \\
63007\end{array}$ & $8 / 12 / 2004$ & 8 & $\begin{array}{l}\text { (Ishida } \\
, 2007)\end{array}$ \\
\hline $\begin{array}{c}\text { Japan } \\
\text { Tobacco Company }\end{array}$ & $\begin{array}{l}2016 / 00 \\
83737\end{array}$ & $3 / 27 / 2014$ & 5 & $\begin{array}{l}\text { (Imaya } \\
\text { ma et al., } \\
2016)\end{array}$ \\
\hline Syngenta & $\begin{array}{l}2015 / 01 \\
13681\end{array}$ & $10 / 23 / 2013$ & 1 & $\begin{array}{l}\text { (Zhong } \\
\text {, 2015) }\end{array}$ \\
\hline $\begin{array}{l}\text { Iowa State } \\
\text { University Plant }\end{array}$ & $\begin{array}{r}10.1104 \\
\text { /pp.000653 }\end{array}$ & May, 2002 & $\mathrm{~N} / \mathrm{A}$ & $\begin{array}{r}\text { (Fram } \\
\text { e et al., 2002) }\end{array}$ \\
\hline
\end{tabular}




\begin{tabular}{|c|r|r|r|r|}
\hline $\begin{array}{c}\text { Transformation } \\
\text { Core }\end{array}$ & 10.1007 & 2014 & N/A & (Lee \\
\hline $\begin{array}{c}\text { University } \\
\text { of Missouri }\end{array}$ & 1978-1-62703- & & & and Zhang, \\
Transformation & $715-0 \_22$ & & & 2014) \\
Core & & & & \\
\hline
\end{tabular}

Table 1 Literature Sources for Protocol Review.

Representative patents and literature from various companies, institutions, and time periods. International filings indicates the number of countries a given patent family was filed in. 


\begin{tabular}{|c|c|c|c|c|c|c|c|c|c|}
\hline \multicolumn{10}{|c|}{ Inoculation Media } \\
\hline Source & lowa State & Japan Tobacco & Japan Tobacco & Japan Tobacco & Missouri & Monsanto & Monsanto & Monsanto & Monsanto \\
\hline Date & 2002 & $2 / 28 / 96$ & $8 / 12 / 04$ & $3 / 27 / 14$ & 2014 & $6 / 1 / 04$ & $8 / 31 / 07$ & $3 / 22 / 10$ & $3 / 8 / 13$ \\
\hline Countries Filed & NA & NA & 8 & 5 & NA & 1 & 10 & 1 & 10 \\
\hline Name & Infection & LS-AS & LS-Inf & LS-Inf & PHI-A & 1/2 MSPL & N/A & 1/2 MSPL & Not Listed \\
\hline $2,4 D$ & $1.5 \mathrm{mg} / \mathrm{L}$ & $1.5 \mathrm{mg} / \mathrm{L}$ & $1.5 \mathrm{mg} / \mathrm{L}$ & $1.5 \mathrm{mg} / \mathrm{L}$ & $1.5 \mathrm{mg} / \mathrm{L}$ & & & & \\
\hline Acetosyringone & $100 \mathrm{uM}$ & & $100 \mathrm{uM}$ & $100 \mathrm{uM}$ & $100 \mathrm{uM}$ & $200 \mathrm{uM}$ & & 200 uM & \\
\hline Casamino Acid & & $1 \mathrm{~g} / \mathrm{L}$ & $1 \mathrm{~g} / \mathrm{L}$ & $1 \mathrm{~g} / \mathrm{L}$ & & & & & \\
\hline \multicolumn{10}{|l|}{ Dicamba } \\
\hline FeEDTA & & & & $1 \mathrm{mM}$ & & & & & \\
\hline Glucose & $36 \mathrm{~g} / \mathrm{L}$ & $36 \mathrm{~g} / \mathrm{L}$ & $36 \mathrm{~g} / \mathrm{L}$ & $36.04 \mathrm{~g} / \mathrm{L}$ & $36 \mathrm{~g} / \mathrm{L}$ & $36 \mathrm{~g} / \mathrm{L}$ & & $36 \mathrm{~g} / \mathrm{L}$ & \\
\hline Glycine & & & & & $2 \mathrm{mg} / \mathrm{L}$ & $2 \mathrm{mg} / \mathrm{L}$ & & $2 \mathrm{mg} / \mathrm{L}$ & \\
\hline L-Proline & $.7 \mathrm{~g} / \mathrm{L}$ & & & & $.7 \mathrm{~g} / \mathrm{L}$ & $115 \mathrm{mg} / \mathrm{L}$ & & $115 \mathrm{mg} / \mathrm{L}$ & \\
\hline LS Salt & & Yes & Yes & Yes & & & & & \\
\hline MES Salts & & & & & $.5 \mathrm{~g} / \mathrm{L}$ & & & & \\
\hline \multicolumn{10}{|l|}{ MS Iron } \\
\hline MS Salts & & & & & & $68.5 \mathrm{~g} / \mathrm{L}$ & & $68.5 \mathrm{~g} / \mathrm{L}$ & \\
\hline Myo-Inositol & & $100 \mathrm{mg} / \mathrm{L}$ & $100 \mathrm{mg} / \mathrm{L}$ & $100 \mathrm{mg} / \mathrm{L}$ & & $100 \mathrm{mg} / \mathrm{L}$ & & $100 \mathrm{mg} / \mathrm{L}$ & \\
\hline N6 Salts & Yes & & & & $2 \mathrm{~g} / \mathrm{L}$ & & & & \\
\hline N6 Vitamin & Yes & & & & & & & & \\
\hline Nicotinic Acid & & $.5 \mathrm{mg} / \mathrm{L}$ & $.5 \mathrm{mg} / \mathrm{L}$ & $.5 \mathrm{mg} / \mathrm{L}$ & $.5 \mathrm{mg} / \mathrm{L}$ & $.5 \mathrm{mg} / \mathrm{L}$ & & $.5 \mathrm{mg} / \mathrm{L}$ & \\
\hline $\mathrm{pH}$ & & 5.2 & 5.2 & 5.2 & 5.2 & & & & \\
\hline Pyridoxine $\mathrm{HCL}$ & & $.5 \mathrm{mg} / \mathrm{L}$ & $.5 \mathrm{mg} / \mathrm{L}$ & $.5 \mathrm{mg} / \mathrm{L}$ & $.5 \mathrm{mg} / \mathrm{L}$ & $.5 \mathrm{mg} / \mathrm{L}$ & & $.5 \mathrm{mg} / \mathrm{L}$ & \\
\hline Sucrose & $68.4 \mathrm{~g} / \mathrm{L}$ & $68.5 \mathrm{~g} / \mathrm{L}$ & $68.5 \mathrm{~g} / \mathrm{L}$ & $68.46 \mathrm{~g} / \mathrm{L}$ & $68.5 \mathrm{~g} / \mathrm{L}$ & $68.6 \mathrm{~g} / \mathrm{L}$ & & $68.6 \mathrm{~g} / \mathrm{L}$ & \\
\hline Thiamine HCL & & $1 \mathrm{mg} / \mathrm{L}$ & $1 \mathrm{mg} / \mathrm{L}$ & $.1 \mathrm{mg} / \mathrm{L}$ & $1 \mathrm{mg} / \mathrm{L}$ & $.1 \mathrm{mg} / \mathrm{L}$ & & $.1 \mathrm{mg} / \mathrm{L}$ & \\
\hline Time & $5 \mathrm{Min}$ & $5 \mathrm{Min}$ & $5 \mathrm{Min}$ & $5 \mathrm{Min}$ & $5 \mathrm{Min}$ & 5-20 Min & & 5-20 Min & \\
\hline
\end{tabular}

Table 2 Inoculation Media

$\tilde{D}$ 


\begin{tabular}{|c|c|c|c|c|c|c|c|c|c|}
\hline \multicolumn{10}{|c|}{ Inoculation Media } \\
\hline Source & Monsanto & Pioneer & Pioneer & Pioneer & Pioneer & Pioneer & Pioneer & Syngenta & Consensus \\
\hline Date & $7 / 18 / 13$ & $1 / 21 / 09$ & $1 / 26 / 10$ & $1 / 22 / 10$ & $12 / 30 / 10$ & $3 / 13 / 13$ & $9 / 6 / 16$ & $4 / 23 / 15$ & \\
\hline Countries Filed & 10 & 4 & 1 & 1 & 7 & 14 & NA & 1 & \\
\hline Name & Not Listed & 700 & Phil & $561 Q$ & Not Given & Not listed & 700 & Inoculation & NG-I \\
\hline 2,4D & & & $1.5 \mathrm{mg} / \mathrm{L}$ & $1.5 \mathrm{mg} / \mathrm{L}$ & $1.5 \mathrm{mg} / \mathrm{L}$ & & $1.5 \mathrm{mg} / \mathrm{L}$ & & $1.5 \mathrm{mg} / \mathrm{L}$ \\
\hline Acetosyringone & & & $100 \mathrm{uM}$ & & & & & & $100 \mathrm{uM}$ \\
\hline Casamino Acid & & $1 \mathrm{~g} / \mathrm{L}$ & $1 \mathrm{~g} / \mathrm{L}$ & & & & & & $1 \mathrm{~g} / \mathrm{L}$ \\
\hline Dicamba & & & & & & & & $5 \mathrm{mg} / \mathrm{L}$ & \\
\hline \multicolumn{10}{|l|}{ FeEDTA } \\
\hline Glucose & & $36 \mathrm{~g} / \mathrm{L}$ & $36 \mathrm{~g} / \mathrm{L}$ & $36 \mathrm{~g} / \mathrm{L}$ & $36 \mathrm{~g} / \mathrm{L}$ & & $36 \mathrm{~g} / \mathrm{L}$ & $36 \mathrm{~g} / \mathrm{L}$ & $36 \mathrm{~g} / \mathrm{L}$ \\
\hline \multicolumn{10}{|l|}{ Glycine } \\
\hline L-Proline & & & & $.69 \mathrm{~g} / \mathrm{L}$ & $.69 \mathrm{~g} / \mathrm{L}$ & & & & \\
\hline LS Salt & & & & & & & & Yes & \\
\hline \multicolumn{10}{|l|}{ MES Salts } \\
\hline MS Iron & & & & & & & Yes & & \\
\hline MS Salts & & $4.3 \mathrm{~g} / \mathrm{L}$ & $4.3 \mathrm{~g} / \mathrm{L}$ & & & & Yes & & $4.3 \mathrm{~g} / \mathrm{L}$ \\
\hline Myo-Inositol & & $.1 \mathrm{~g} / \mathrm{L}$ & $.1 \mathrm{~g} / \mathrm{L}$ & & & & & & $100 \mathrm{mg} / \mathrm{L}$ \\
\hline N6 Salts & & & & $4 \mathrm{~g} / \mathrm{L}$ & $4 \mathrm{~g} / \mathrm{L}$ & & & & \\
\hline N6 Vitamin & & & & Yes & Yes & & & & \\
\hline Nicotinic Acid & & $.5 \mathrm{mg} / \mathrm{L}$ & $.5 \mathrm{mg} / \mathrm{L}$ & & & & & & $.5 \mathrm{mg} / \mathrm{L}$ \\
\hline pH & & 5.2 & 5.2 & & 5.2 & & 5.8 & & 5.2 \\
\hline Pyridoxine HCL & & $0.5 \mathrm{mg} / \mathrm{L}$ & $.5 \mathrm{mg} / \mathrm{L}$ & & & & & & $.5 \mathrm{mg} / \mathrm{L}$ \\
\hline Sucrose & & $68.5 \mathrm{~g} / \mathrm{L}$ & $68.5 \mathrm{~g} / \mathrm{L}$ & $68.5 \mathrm{~g} / \mathrm{L}$ & $68.5 \mathrm{~g} / \mathrm{L}$ & & $68.5 \mathrm{~g} / \mathrm{L}$ & $68.5 \mathrm{~g} / \mathrm{L}$ & $68.5 \mathrm{~g} / \mathrm{L}$ \\
\hline Thiamine $\mathrm{HCL}$ & & $10 \mathrm{mg} / \mathrm{L}$ & $1 \mathrm{mg} / \mathrm{L}$ & $.5 \mathrm{mg} / \mathrm{L}$ & $1 \mathrm{mg} / \mathrm{L}$ & & & & $1 \mathrm{mg} / \mathrm{L}$ \\
\hline Time & & $5 \mathrm{Min}$ & $5 \mathrm{Min}$ & & $5 \mathrm{Min}$ & & $5 \mathrm{Min}$ & & $5 \mathrm{Min}$ \\
\hline
\end{tabular}

Table 2 Inoculation Media Continued 


\begin{tabular}{|c|c|c|c|c|c|c|c|c|c|c|c|}
\hline \multicolumn{12}{|c|}{ CoCultivation Media } \\
\hline Source & lowa State & Japan Tobacco & Japan Tobacco & \begin{tabular}{|l|} 
Japan Tobacco \\
\end{tabular} & Missouri & Monsanto & Monsanto & \begin{tabular}{|l|} 
Monsanto \\
\end{tabular} & Monsanto & Monsanto & \begin{tabular}{|l|} 
Pioneer \\
\end{tabular} \\
\hline Date & 2002 & $2 / 28 / 96$ & $8 / 12 / 04$ & $3 / 27 / 14$ & 2014 & $6 / 1 / 04$ & $8 / 31 / 07$ & $3 / 22 / 10$ & $3 / 8 / 13$ & $7 / 18 / 13$ & $1 / 21 / 09$ \\
\hline Countries Filed & NA & NA & 8 & 5 & NA & 1 & 10 & 1 & 10 & 10 & 4 \\
\hline Name & \begin{tabular}{|l|} 
Co-Cultivation \\
\end{tabular} & LS-AS & LS-AS & LS-AS & Phi-B & \begin{tabular}{|l|} 
Co-Culture \\
\end{tabular} & \begin{tabular}{|l|}
$1947 / 1898$ \\
\end{tabular} & \begin{tabular}{|l|} 
Co-Culture \\
\end{tabular} & 1947 & 1514 & $710 \mathrm{~B}$ \\
\hline $2,4 \mathrm{D}$ & $1.5 \mathrm{mg} / \mathrm{L}$ & $1.5 \mathrm{mg} / \mathrm{L}$ & $1.5 \mathrm{mg} / \mathrm{L}$ & $1.5 \mathrm{mg} / \mathrm{L}$ & $1.5 \mathrm{mg} / \mathrm{L}$ & $3 \mathrm{mg} / \mathrm{L}$ & $.5 \mathrm{mg} / \mathrm{L}$ & $3 \mathrm{mg} / \mathrm{L}$ & $.5 \mathrm{mg} / \mathrm{L}$ & $.5 \mathrm{mg} / \mathrm{L}$ & $2 \mathrm{mg} / \mathrm{L}$ \\
\hline 6-benzylaminopurine & & & & & & & & & $.01 \mathrm{mg} / \mathrm{L}$ & & \\
\hline Acetosyringone & $100 \mathrm{uM}$ & $100 \mathrm{uM}$ & $100 \mathrm{uM}$ & $100 \mathrm{uM}$ & $100 \mathrm{uM}$ & & $200 \mathrm{uM}$ & & $200 \mathrm{uM}$ & & $100 \mathrm{mM}$ \\
\hline Agar & & $8 \mathrm{~g} / \mathrm{L}$ & Yes & Yes & $5 \mathrm{~g} / \mathrm{L}$ & Yes & & Yes & & & Yes \\
\hline Ascorbic Acid & & & & & & & & & & & $10 \mathrm{mg} / \mathrm{L}$ \\
\hline Carbenicillin & & & & & & & $50 \mathrm{mg} / \mathrm{L}$ & & $50 \mathrm{mg} / \mathrm{L}$ & $500 \mathrm{mg} / \mathrm{L}$ & \\
\hline Casamino Acids & & & & & & & $.5 \mathrm{~g} / \mathrm{L}$ & & $.5 \mathrm{~g} / \mathrm{L}$ & $.5 \mathrm{~g} / \mathrm{L}$ & \\
\hline Copper Sulfate & & & 5 uM & 5 uM & & & & & & & \\
\hline Cysteine & $300 \mathrm{mg} / \mathrm{L}$ & & & & $.4 \mathrm{~g} / \mathrm{L}$ & & & & & & \\
\hline \multicolumn{12}{|l|}{ Dicamba } \\
\hline DTT & & & & & $.154 \mathrm{~g} / \mathrm{L}$ & & & & & & \\
\hline FeEDA & & & & $1 \mathrm{mM}$ & & & & & & & \\
\hline Gelrite & $3 \mathrm{~g} / \mathrm{L}$ & & & & & & & & & & \\
\hline \multicolumn{12}{|l|}{ Gelzan } \\
\hline Glucose & & $10 \mathrm{~g} / \mathrm{L}$ & $10 \mathrm{~g} / \mathrm{L}$ & $10 \mathrm{~g} / \mathrm{L}$ & & $10 \mathrm{~g} / \mathrm{L}$ & & $10 \mathrm{~g} / \mathrm{L}$ & & & $10 \mathrm{~g} / \mathrm{L}$ \\
\hline Glycine & & & & & & $2 \mathrm{mg} / \mathrm{L}$ & & $2 \mathrm{mg} / \mathrm{L}$ & & & \\
\hline Glycine & & & & & $2 \mathrm{mg} / \mathrm{L}$ & $2 \mathrm{mg} / \mathrm{L}$ & & $2 \mathrm{mg} / \mathrm{L}$ & & & \\
\hline \multicolumn{12}{|l|}{ L-Cysteine } \\
\hline L-Proline & $.7 \mathrm{~g} / \mathrm{L}$ & $.7 \mathrm{~g} / \mathrm{L}$ & $.7 \mathrm{~g} / \mathrm{L}$ & $.7 \mathrm{~g} / \mathrm{L}$ & $.7 \mathrm{~g} / \mathrm{L}$ & $115 \mathrm{mg} / \mathrm{L}$ & $1.38 \mathrm{~g} / \mathrm{L}$ & \begin{tabular}{|l|}
$115 \mathrm{mg} / \mathrm{L}$ \\
\end{tabular} & $1.38 \mathrm{~g} / \mathrm{L}$ & $1.38 \mathrm{~g} / \mathrm{L}$ & $.7 \mathrm{~g} / \mathrm{L}$ \\
\hline Low EEO Agarose & & & & & & & $5.5 \mathrm{~g} / \mathrm{L}$ & & $5.5 \mathrm{~g} / \mathrm{L}$ & & \\
\hline LS Salt & & Yes & Yes & Yes & & & & & & & \\
\hline MES Salts & & $500 \mathrm{mg} / \mathrm{L}$ & $500 \mathrm{mg} / \mathrm{L}$ & $.5 \mathrm{~g} / \mathrm{L}$ & $.5 \mathrm{~g} / \mathrm{L}$ & & & & & & $.5 \mathrm{~g} / \mathrm{L}$ \\
\hline MS Salts & & & & & & $2.2 \mathrm{~g} / \mathrm{L}$ & $4.33 \mathrm{~g} / \mathrm{L}$ & $2.2 \mathrm{~g} / \mathrm{L}$ & $4.33 \mathrm{~g} / \mathrm{L}$ & $4.33 \mathrm{~g} / \mathrm{L}$ & $4.3 \mathrm{~g} / \mathrm{L}$ \\
\hline MS Vitamins & & & & & & & Yes & & Yes & Yes & \\
\hline Myo-Inositol & & $100 \mathrm{mg} / \mathrm{L}$ & $100 \mathrm{mg} / \mathrm{L}$ & $100 \mathrm{mg} / \mathrm{L}$ & & $100 \mathrm{mg} / \mathrm{L}$ & & $100 \mathrm{mg} / \mathrm{L}$ & & & $100 \mathrm{mg} / \mathrm{L}$ \\
\hline N6 Salt & Yes & & & & $2 \mathrm{~g} / \mathrm{L}$ & & & & & & \\
\hline N6 Vitamins & Yes & & & & & & & & & & \\
\hline Nicotinic Acid & & $.5 \mathrm{mg} / \mathrm{L}$ & $.5 \mathrm{mg} / \mathrm{L}$ & $.5 \mathrm{mg} / \mathrm{L}$ & $.5 \mathrm{mg} / \mathrm{L}$ & $.5 \mathrm{mg} / \mathrm{L}$ & & $.5 \mathrm{mg} / \mathrm{L}$ & & & $.5 \mathrm{mg} / \mathrm{L}$ \\
\hline $\mathrm{pH}$ & & 5.8 & 5.8 & 5.8 & 5.8 & 5.2 & 5.8 & & 5.8 & 5.8 & 5.8 \\
\hline Phytagel & & & & & & & & & & $3 \mathrm{~g} / \mathrm{L}$ & \\
\hline Pyridoxine HCL & & $.5 \mathrm{mg} / \mathrm{L}$ & $.5 \mathrm{mg} / \mathrm{L}$ & $.5 \mathrm{mg} / \mathrm{L}$ & $.5 \mathrm{mg} / \mathrm{L}$ & $.5 \mathrm{mg} / \mathrm{L}$ & & $.5 \mathrm{mg} / \mathrm{L}$ & & & $.5 \mathrm{mg} / \mathrm{L}$ \\
\hline Selection & & & & & & & & & & $0.1 \mathrm{mM}$ Glyphosate & \\
\hline SilverNitrate & $.85 \mathrm{mg} / \mathrm{L}$ & & & $5 \mathrm{uM}$ & $.85 \mathrm{mg} / \mathrm{L}$ & $1.7 \mathrm{mg} / \mathrm{L}$ & $3.4 \mathrm{mg} / \mathrm{L}$ & $1.7 \mathrm{mg} / \mathrm{L}$ & $3.4 \mathrm{mg} / \mathrm{L}$ & $3.4 \mathrm{mg} / \mathrm{L}$ & \\
\hline Sucrose & $30 \mathrm{~g} / \mathrm{L}$ & $20 \mathrm{~g} / \mathrm{L}$ & $20 \mathrm{~g} / \mathrm{L}$ & $20 \mathrm{~g} / \mathrm{L}$ & $30 \mathrm{~g} / \mathrm{L}$ & $20 \mathrm{~g} / \mathrm{L}$ & $30 \mathrm{~g} / \mathrm{L}$ & $20 \mathrm{~g} / \mathrm{L}$ & $30 \mathrm{~g} / \mathrm{L}$ & $30 \mathrm{~g} / \mathrm{L}$ & $20 \mathrm{~g} / \mathrm{L}$ \\
\hline \multicolumn{12}{|l|}{ Ticarcillin } \\
\hline Thiamine $\mathrm{HCL}$ & & $1 \mathrm{mg} / \mathrm{L}$ & $1 \mathrm{mg} / \mathrm{L}$ & $1 \mathrm{mg} / \mathrm{L}$ & $1 \mathrm{mg} / \mathrm{L}$ & $.6 \mathrm{mg} / \mathrm{L}$ & $.5 \mathrm{mg} / \mathrm{L}$ & $.6 \mathrm{mg} / \mathrm{L}$ & $.5 \mathrm{mg} / \mathrm{L}$ & $.5 \mathrm{mg} / \mathrm{L}$ & $10 \mathrm{mg} / \mathrm{L}$ \\
\hline \multicolumn{12}{|l|}{ Thymidine } \\
\hline Time & $3 d-20 c$ & $3 d-25 C$ & $3 d-25 C$ & $7 d-25 c$ & $3 d-20 c$ & $1 d-23 c$ & $1-3 d-23 c$ & $1 d-23 C$ & $14 d-23 C$ & $24 \mathrm{hr}-23 \mathrm{C}$ & $3 d-25 C$ \\
\hline & & & & & & & $7-14 d-30 c$ & & $1 \mathrm{~d}-30 \mathrm{c}$ & & \\
\hline
\end{tabular}

Table 3 CoCultivation Media 


\begin{tabular}{|c|c|c|c|c|c|c|c|}
\hline \multicolumn{8}{|c|}{ CoCultivation Media } \\
\hline Source & \begin{tabular}{|l|} 
Pioneer \\
\end{tabular} & \begin{tabular}{|l|} 
Pioneer \\
\end{tabular} & Pioneer & Pioneer & \begin{tabular}{|l|} 
Pioneer \\
\end{tabular} & Syngenta & Consensus \\
\hline Date & $1 / 26 / 10$ & $1 / 22 / 10$ & $12 / 30 / 10$ & $3 / 13 / 13$ & 9/6/16 & $4 / 23 / 15$ & \\
\hline Countries Filed & 1 & 1 & 7 & 14 & NA & 1 & \\
\hline Name & Phit & 562P & Not Listed & Phit & 7101 & AW5Dicamba100Ti & NG-CC \\
\hline $2,4 \mathrm{D}$ & $2 \mathrm{mg} / \mathrm{L}$ & $2 \mathrm{mg} / \mathrm{L}$ & $1.5 \mathrm{mg} / \mathrm{L}$ & $2 \mathrm{mg} / \mathrm{L}$ & $2 \mathrm{mg} / \mathrm{L}$ & & $1.5 \mathrm{mg} / \mathrm{L}$ \\
\hline 6-benzylaminopurine & & & & & & & $.01 \mathrm{mg} / \mathrm{L}$ \\
\hline Acetosyringone & $100 \mathrm{uM}$ & $100 \mathrm{uM}$ & $100 \mathrm{mM}$ & $100 \mathrm{uM}$ & $100 \mathrm{uM}$ & & $100 \mathrm{uM}$ \\
\hline Agar & $8 \mathrm{~g} / \mathrm{L}$ & & & $8 \mathrm{~g} / \mathrm{L}$ & & & $8 \mathrm{~g} / \mathrm{L}$ \\
\hline Ascorbic Acid & $10 \mathrm{mg} / \mathrm{L}$ & & & $10 \mathrm{mg} / \mathrm{L}$ & & & \\
\hline \multicolumn{8}{|l|}{ Carbenicillin } \\
\hline \multicolumn{8}{|l|}{ Casamino Acids } \\
\hline Copper Sulfate & $.1 \mathrm{uM}$ & & & $.1 \mathrm{um}$ & & & $.1 \mathrm{uM}$ \\
\hline \multicolumn{8}{|l|}{ Cysteine } \\
\hline Dicamba & & & & & & $5 \mathrm{mg} / \mathrm{ml}$ & \\
\hline \multicolumn{8}{|l|}{ DTT } \\
\hline \multicolumn{8}{|l|}{ FeEDA } \\
\hline Gelrite & & $3 \mathrm{~g} / \mathrm{L}$ & $3 \mathrm{~g} / \mathrm{L}$ & & & & \\
\hline Gelzan & & & & & & $2.4 \mathrm{~g} / \mathrm{L}$ & \\
\hline Glucose & $10 \mathrm{~g} / \mathrm{L}$ & & & $10 \mathrm{~g} / \mathrm{L}$ & $10 \mathrm{~g} / \mathrm{L}$ & & $10 \mathrm{~g} / \mathrm{L}$ \\
\hline \multicolumn{8}{|l|}{ Glycine } \\
\hline \multicolumn{8}{|l|}{ Glycine } \\
\hline \multicolumn{8}{|l|}{ L-Cysteine } \\
\hline L-Proline & $.7 \mathrm{~g} / \mathrm{L}$ & $.69 \mathrm{~g} / \mathrm{L}$ & $.69 \mathrm{~g} / \mathrm{L}$ & $700 \mathrm{mg} / \mathrm{L}$ & & $1.38 \mathrm{~g} / \mathrm{L}$ & $700 \mathrm{mg} / \mathrm{L}$ \\
\hline \multicolumn{8}{|l|}{ Low EEO Agarose } \\
\hline \multicolumn{8}{|l|}{ LS Salt } \\
\hline MES Salts & $.5 \mathrm{~g} / \mathrm{L}$ & & & $.5 \mathrm{~g} / \mathrm{L}$ & & & $.5 \mathrm{~g} / \mathrm{L}$ \\
\hline MS Salts & $4.3 \mathrm{mg} / \mathrm{L}$ & & & $4.3 \mathrm{mg} / \mathrm{L}$ & Yes & $4.3 \mathrm{~g} / \mathrm{L}$ & $4.3 \mathrm{~g} / \mathrm{L}$ \\
\hline MS Vitamins & & & & & & Yes & \\
\hline Myo-Inositol & $100 \mathrm{mg} / \mathrm{L}$ & & & $100 \mathrm{mg} / \mathrm{L}$ & & & $100 \mathrm{mg} / \mathrm{L}$ \\
\hline N6 Salt & & $4 \mathrm{~g} / \mathrm{L}$ & $4 \mathrm{~g} / \mathrm{L}$ & & & & \\
\hline N6 Vitamins & & Yes & Yes & & & & \\
\hline Nicotinic Acid & $.5 \mathrm{mg} / \mathrm{L}$ & & & $.5 \mathrm{mg} / \mathrm{L}$ & & & $.5 \mathrm{mg} / \mathrm{L}$ \\
\hline $\mathrm{pH}$ & 5.8 & 5.8 & & & 5.8 & & 5.8 \\
\hline \multicolumn{8}{|l|}{ Phytagel } \\
\hline Pyridoxine $\mathrm{HCL}$ & $.5 \mathrm{mg} / \mathrm{L}$ & & & $.5 \mathrm{mg} / \mathrm{L}$ & & & $.5 \mathrm{mg} / \mathrm{L}$ \\
\hline \multicolumn{8}{|l|}{ Selection } \\
\hline SilverNitrate & & $.85 \mathrm{mg} / \mathrm{L}$ & $.85 \mathrm{mg} / \mathrm{L}$ & & & & $1.7 \mathrm{mg} / \mathrm{L}$ \\
\hline Sucrose & $20 \mathrm{~g} / \mathrm{L}$ & $30 \mathrm{~g} / \mathrm{L}$ & $30 \mathrm{~g} / \mathrm{L}$ & $20 \mathrm{~g} / \mathrm{L}$ & $20 \mathrm{~g} / \mathrm{L}$ & $30 \mathrm{~g} / \mathrm{L}$ & $25 \mathrm{~g} / \mathrm{L}$ \\
\hline Ticarcillin & & & & & & $100 \mathrm{mg} / \mathrm{L}$ & \\
\hline Thiamine $\mathrm{HCL}$ & $1 \mathrm{mg} / \mathrm{L}$ & $.5 \mathrm{mg} / \mathrm{L}$ & $1 \mathrm{mg} / \mathrm{L}$ & $1 \mathrm{mg} / \mathrm{L}$ & & & $1 \mathrm{mg} / \mathrm{L}$ \\
\hline Thymidine & & & & & $50 \mathrm{mg} / \mathrm{L}$ & & \\
\hline Time & $3 \mathrm{~d}$ & & $3 d-20 c$ & $3 d-21 C$ & $3 d-21 c$ & & $3 d-21 c$ \\
\hline & & & $4 d-28 c$ & & & & \\
\hline
\end{tabular}

Table 3 CoCultivation Media Continued 


\begin{tabular}{|c|c|c|c|c|c|c|c|c|}
\hline \multicolumn{9}{|c|}{ Resting Media } \\
\hline Source & lowa State & Japan Tobacco & Japan Tobacco & Japan Tobacco & Missouri & Monsanto & Monsanto & Monsanto \\
\hline Date & 2002 & $2 / 28 / 96$ & $8 / 12 / 04$ & $3 / 27 / 14$ & 2014 & $6 / 1 / 04$ & $8 / 31 / 07$ & $3 / 22 / 10$ \\
\hline Countries Filed & NA & NA & 8 & 5 & NA & 1 & 10 & 1 \\
\hline Name & \begin{tabular}{|l|} 
Resting Medium \\
\end{tabular} & None & None & None & Phi-C & NONE & 1316 Proliferation & NONE \\
\hline $2,4 \mathrm{D}$ & $1.5 \mathrm{mg} / \mathrm{L}$ & & & & $1.5 \mathrm{mg} / \mathrm{L}$ & & $.5 \mathrm{mg} / \mathrm{L}$ & \\
\hline 6-benzylaminopurine & & & & & & & $.01 \mathrm{mg} / \mathrm{L}$ & \\
\hline Agar & $8 \mathrm{~g} / \mathrm{L}$ & & & & & & & \\
\hline \multicolumn{9}{|l|}{ Ascobic Acid } \\
\hline Carb & & & & & & & $500 \mathrm{mg} / \mathrm{L}$ & \\
\hline Casamino Acid & & & & & & & $.5 \mathrm{~g} / \mathrm{L}$ & \\
\hline \multicolumn{9}{|l|}{ Caseine Hydrolysate } \\
\hline Cefotaxime & $100 \mathrm{mg} / \mathrm{L}$ & & & & $250 \mathrm{mg} / \mathrm{L}$ & & & \\
\hline \multicolumn{9}{|l|}{ Copper Sulfate } \\
\hline \multicolumn{9}{|l|}{ Dicamba } \\
\hline Gelrite & & & & & $3 \mathrm{~g} / \mathrm{L}$ & & & \\
\hline \multicolumn{9}{|l|}{ Glucose } \\
\hline Glycine & & & & & $2 \mathrm{mg} / \mathrm{L}$ & & & \\
\hline L-Proline & $.7 \mathrm{~g} / \mathrm{L}$ & & & & $.7 \mathrm{~g} / \mathrm{L}$ & & $1.38 \mathrm{~g} / \mathrm{L}$ & \\
\hline \multicolumn{9}{|l|}{ Maltose } \\
\hline MES Salts & $.5 \mathrm{~g} / \mathrm{L}$ & & & & $.5 \mathrm{~g} / \mathrm{L}$ & & & \\
\hline MS Salts & & & & & & & $4.33 \mathrm{~g} / \mathrm{L}$ & \\
\hline MS Vitamin & & & & & & & Yes & \\
\hline \multicolumn{9}{|l|}{ Myo-Inositol } \\
\hline N6 Salts & Yes & & & & $4 \mathrm{~g} / \mathrm{L}$ & & & \\
\hline N6 Vitamins & Yes & & & & & & & \\
\hline Nicotinic Acid & & & & & $.5 \mathrm{mg} / \mathrm{L}$ & & & \\
\hline pH & & & & & & & 5.8 & \\
\hline Phytagel & & & & & & & $3 \mathrm{~g} / \mathrm{L}$ & \\
\hline Pyridoxine $\mathrm{HCL}$ & & & & & $.5 \mathrm{mg} / \mathrm{L}$ & & & \\
\hline Silver Nitrate & $.85 \mathrm{mg} / \mathrm{L}$ & & & & $.85 \mathrm{mg} / \mathrm{L}$ & & $3.4 \mathrm{mg} / \mathrm{L}$ & \\
\hline Sucrose & $30 \mathrm{~g} / \mathrm{L}$ & & & & $30 \mathrm{~g} / \mathrm{L}$ & & $30 \mathrm{~g} / \mathrm{L}$ & \\
\hline Thiamine $\mathrm{HCL}$ & & & & & $1 \mathrm{mg} / \mathrm{L}$ & & $.5 \mathrm{mg} / \mathrm{L}$ & \\
\hline Vancomycin & $100 \mathrm{mg} / \mathrm{L}$ & & & & & & & \\
\hline Time & & & & & $7 d-28 c$ & Skip & $10 \mathrm{~d}$ & Skip \\
\hline
\end{tabular}

Table 4 Resting Media 


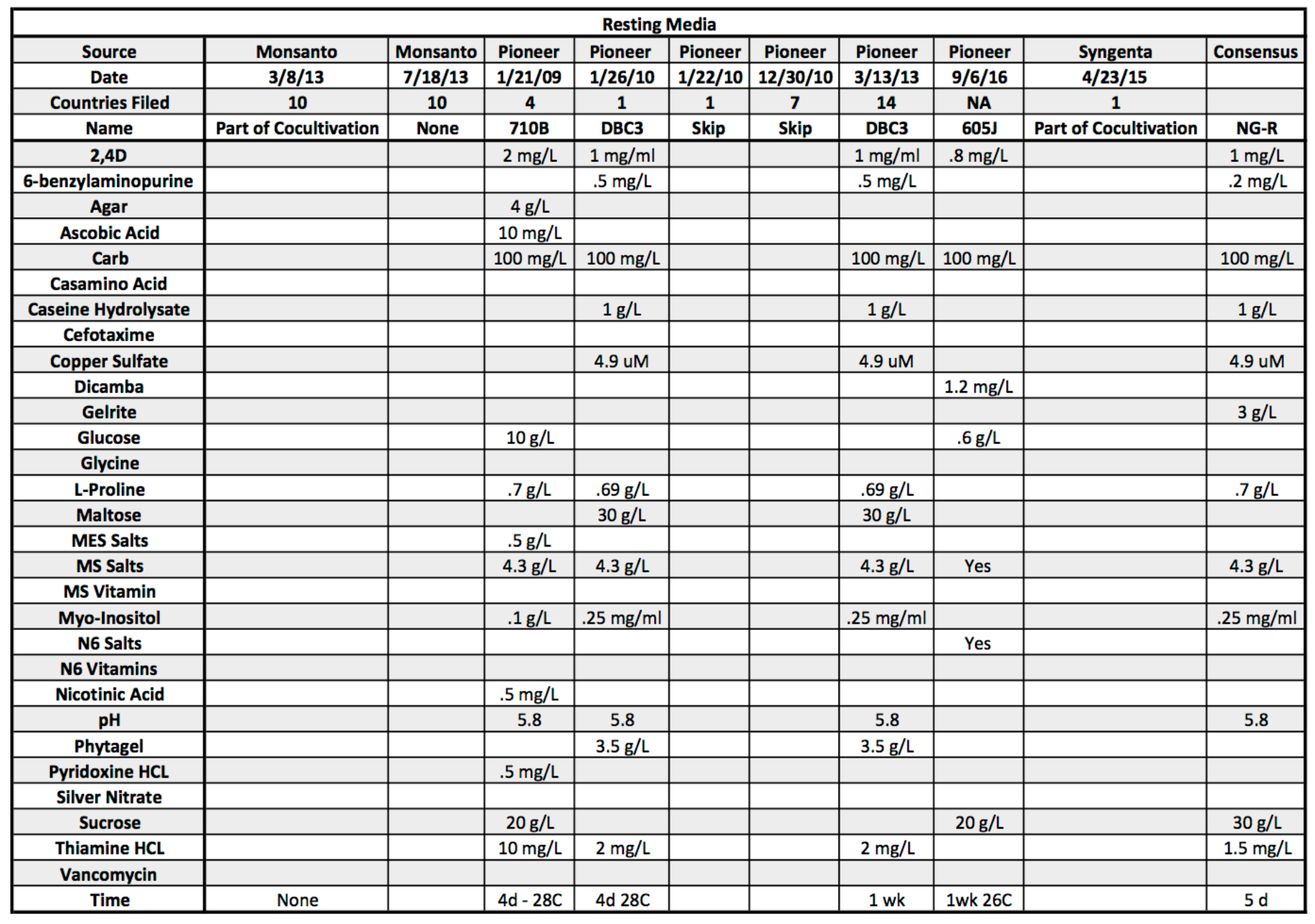

Table 4 Resting Media Continued 


\begin{tabular}{|c|c|c|c|c|c|c|c|c|c|c|}
\hline \multicolumn{11}{|c|}{ Selection Media } \\
\hline Source & lowa State & Japan Tobacco & Japan Tobacco & Japan Tobacco & Missouri & Monsanto & Monsanto & Monsanto & \multicolumn{2}{|c|}{ Monsanto } \\
\hline Date & 2002 & $2 / 28 / 96$ & $8 / 12 / 04$ & $3 / 27 / 14$ & 2014 & $6 / 1 / 04$ & $8 / 31 / 07$ & $3 / 22 / 10$ & \multirow{2}{*}{\multicolumn{2}{|c|}{$\frac{3 / 8 / 13}{10}$}} \\
\hline Countries Filed & NA & NA & 8 & 5 & NA & 1 & 10 & 1 & & \\
\hline Name & Selection Medium & LSD1.5 & LSD & LSD1.5A/B & PhiD & Induction MS & Preregeneration 1844 & Induction MS & 2202 & 2067 \\
\hline $2,4 \mathrm{D}$ & $1.5 \mathrm{mg} / \mathrm{L}$ & $1.5 \mathrm{mg} / \mathrm{L}$ & $1.5 \mathrm{mg} / \mathrm{L}$ & $1.5 \mathrm{mg} / \mathrm{L}$ & $1.5 \mathrm{mg} / \mathrm{L}$ & $.5 \mathrm{mg} / \mathrm{L}$ & $.2 \mathrm{mg} / \mathrm{L}$ & $.5 \mathrm{mg} / \mathrm{L}$ & $.2 \mathrm{mg} / \mathrm{L}$ & \\
\hline 6-benzylaminopurine & & & & & & & & & $.01 \mathrm{mg} / \mathrm{L}$ & \\
\hline Absiscic Acid & & & & & & & $.26 \mathrm{mg} / \mathrm{L}$ & & & \\
\hline Agar & $8 \mathrm{~g} / \mathrm{L}$ & $8 \mathrm{~g} / \mathrm{L}$ & $8 \mathrm{~g} / \mathrm{L}$ & $8 \mathrm{~g} / \mathrm{L}$ & & & & & & \\
\hline Ascobic Acid & & & & & & & & & & \\
\hline CA Pantothenate & & & & & & $.25 \mathrm{mg} / \mathrm{L}$ & & $.25 \mathrm{mg} / \mathrm{L}$ & & \\
\hline Carb & & & & $250 \mathrm{mg} / \mathrm{L}$ & & $500 \mathrm{mg} / \mathrm{L}$ & $500 \mathrm{mg} / \mathrm{L}$ & $500 \mathrm{mg} / \mathrm{L}$ & $500 \mathrm{mg} / \mathrm{L}$ & $500 \mathrm{mg} / \mathrm{L}$ \\
\hline Casamino Acids & & & & & & $50 \mathrm{mg} / \mathrm{L}$ & & $50 \mathrm{mg} / \mathrm{L}$ & $.5 \mathrm{~g} / \mathrm{L}$ & \\
\hline \begin{tabular}{|l} 
Caseine Hydrolysate \\
\end{tabular} & & & & & & & & & & \\
\hline Cefotaxime & $100 \mathrm{mg} / \mathrm{L}$ & $250 \mathrm{mg} / \mathrm{L}$ & & $100 \mathrm{mg} / \mathrm{L}$ & $250 \mathrm{mg} / \mathrm{L}$ & & & & & \\
\hline Copper Sulfate & & & & & & & & & & \\
\hline Dicamba & & & & & & & & & & \\
\hline FeEDTA & & & & $1 \mathrm{mM}$ & & & & & & \\
\hline Gelrite & & & & & $3 \mathrm{~g} / \mathrm{L}$ & & & & & \\
\hline Glucose & & & & & & & & & & \\
\hline Glycine & & & & & $2 \mathrm{mg} / \mathrm{L}$ & & & & & \\
\hline L-Proline & $.7 \mathrm{~g} / \mathrm{L}$ & $.7 \mathrm{~g} / \mathrm{L}$ & $.7 \mathrm{~g} / \mathrm{L}$ & $.7 \mathrm{~g} / \mathrm{L}$ & $.7 \mathrm{~g} / \mathrm{L}$ & $1.36 \mathrm{~g} / \mathrm{L}$ & & $1.36 \mathrm{~g} / \mathrm{L}$ & & \\
\hline LS Salt & & Yes & Yes & Yes & & & & & & \\
\hline Maltose & & & & & & & & & & \\
\hline MES Salts & $.5 \mathrm{~g} / \mathrm{L}$ & $.5 \mathrm{~g} / \mathrm{L}$ & $.5 \mathrm{~g} / \mathrm{L}$ & $.5 \mathrm{~g} / \mathrm{L}$ & $.5 \mathrm{~g} / \mathrm{L}$ & & & & & \\
\hline MS Salts & & & & & & $4.4 \mathrm{~g} / \mathrm{L}$ & $4.33 \mathrm{~g} / \mathrm{L}$ & $4.4 \mathrm{~g} / \mathrm{L}$ & $4.33 \mathrm{~g} / \mathrm{L}$ & $4.33 \mathrm{~g} / \mathrm{L}$ \\
\hline MS Vitamins & & & & & & & Yes & & Yes & Yes \\
\hline Myo-inositol & & $100 \mathrm{mg} / \mathrm{L}$ & $100 \mathrm{mg} / \mathrm{L}$ & $100 \mathrm{mg} / \mathrm{L}$ & & & & & & \\
\hline N6 Salt & Yes & & & & $4 \mathrm{~g} / \mathrm{L}$ & & & & & \\
\hline N6 Vitamin & Yes & & & & Yes & & & & & \\
\hline Nicotinic Acid & & $.5 \mathrm{mg} / \mathrm{L}$ & $.5 \mathrm{mg} / \mathrm{L}$ & $.5 \mathrm{mg} / \mathrm{L}$ & $.5 \mathrm{mg} / \mathrm{L}$ & $1.3 \mathrm{mg} / \mathrm{L}$ & & $1.3 \mathrm{mg} / \mathrm{L}$ & & \\
\hline $\mathrm{pH}$ & & 5.8 & & 5.8 & & & 5.8 & & 5.8 & 5.8 \\
\hline Phytagar & & & & & & & $6 \mathrm{~g} / \mathrm{L}$ & & & \\
\hline Phytagel & & & & & & & & & & \\
\hline Pyridoxine $\mathrm{HCl}$ & & $.5 \mathrm{mg} / \mathrm{L}$ & $.5 \mathrm{mg} / \mathrm{L}$ & $.5 \mathrm{mg} / \mathrm{L}$ & $.5 \mathrm{mg} / \mathrm{L}$ & $.25 \mathrm{mg} / \mathrm{L}$ & & $.25 \mathrm{mg} / \mathrm{L}$ & & \\
\hline Selection & $\begin{array}{c}1.5 \mathrm{mg} / \mathrm{L}(2 \mathrm{wks}) \text { then } 3 \\
\mathrm{mg} / \mathrm{L} \text { bialaphos }\end{array}$ & $\begin{array}{c}5 \mathrm{mg} / \mathrm{L}(2 \mathrm{wk}) \text { then } 10 \\
\mathrm{mg} / \mathrm{L} \text { bialaphos }\end{array}$ & & $\begin{array}{c}5 \mathrm{mg} / \mathrm{L} \text { then } 10 \mathrm{mg} / \mathrm{L} \\
\text { bialaphos }\end{array}$ & $\begin{array}{c}1.5 \mathrm{mg} / \mathrm{L}(2 \mathrm{wk}) \text { then } 3 \mathrm{mg} / \mathrm{L} \\
\text { bialaphos }\end{array}$ & & & & & \\
\hline Silver Nitrate & $.85 \mathrm{mg} / \mathrm{L}$ & & & $10 \mathrm{uM}$ & $.85 \mathrm{mg} / \mathrm{L}$ & $1.7 \mathrm{mg} / \mathrm{L}$ & & $1.7 \mathrm{mg} / \mathrm{L}$ & $3.4 \mathrm{mg} / \mathrm{L}$ & \\
\hline Sucrose & $30 \mathrm{~g} / \mathrm{L}$ & $20 \mathrm{~g} / \mathrm{L}$ & $20 \mathrm{~g} / \mathrm{L}$ & $20 \mathrm{~g} / \mathrm{L}$ & $30 \mathrm{~g} / \mathrm{L}$ & $30 \mathrm{~g} / \mathrm{L}$ & $40 \mathrm{~g} / \mathrm{L}$ & $30 \mathrm{~g} / \mathrm{L}$ & $50 \mathrm{~g} / \mathrm{L}$ & $60 \mathrm{~g} / \mathrm{L}$ \\
\hline Thiamine & & $1 \mathrm{mg} / \mathrm{L}$ & $1 \mathrm{mg} / \mathrm{L}$ & $1 \mathrm{mg} / \mathrm{L}$ & $1 \mathrm{mg} / \mathrm{L}$ & $.25 \mathrm{mg} / \mathrm{L}$ & & $.25 \mathrm{mg} / \mathrm{L}$ & $.5 \mathrm{mg} / \mathrm{L}$ & \\
\hline Ticarcillin & & & & & & & & & & \\
\hline Timentin & & & & & & & & & & \\
\hline Vancomycin & $100 \mathrm{mg} / \mathrm{L}$ & & & & & & & & & \\
\hline Time & $2 \mathrm{wk} 1.5 \mathrm{mg} / \mathrm{L}$ bialaphos & $2 \mathrm{wk} 5 \mathrm{mg} / \mathrm{L}$ & $4 \mathrm{wk}$ & $10 \mathrm{~d} 1.5 \mathrm{~A}$ & 2 wks $1.5 \mathrm{mg} / \mathrm{L}$ & $2 w k-27 C$ & $10 \mathrm{~d}$ & $2 w k-27 c$ & $1 \mathrm{wk}-30 \mathrm{c}$ & $2 \mathrm{wk}-30 \mathrm{C}$ \\
\hline & $4 \mathrm{wk} 3 \mathrm{mg} / \mathrm{L}$ & 6 wk $10 \mathrm{mg} / \mathrm{L}$ & & $21 \mathrm{~d} 1.5 \mathrm{~B}$ & $\begin{array}{c}\text { Until Embryoids (at least } 2 \\
\text { months) } 3 \mathrm{mg} / \mathrm{L}\end{array}$ & & & & & $\begin{array}{c}4 \text { wk - 27-30C } \\
\text { Light/Dark }\end{array}$ \\
\hline & & & & $21 \mathrm{~d} 1.5 B$ & & & & & & \\
\hline
\end{tabular}

Table 5 Selection Media 


\begin{tabular}{|c|c|c|c|c|c|c|c|c|c|c|c|}
\hline \multicolumn{12}{|c|}{ Selection Media } \\
\hline Source & Monsanto & Pioneer & Pioneer & Pioneer & Pioneer & Pioneer & Pioneer & \multirow{2}{*}{\multicolumn{3}{|c|}{ Syngenta }} & Consensus \\
\hline Date & $7 / 18 / 13$ & $1 / 21 / 09$ & $1 / 26 / 10$ & $1 / 22 / 10$ & $12 / 30 / 10$ & $3 / 13 / 13$ & $9 / 6 / 16$ & $4 / 23 / 15$ & & & \\
\hline Countries Filed & 10 & 4 & 1 & 1 & 7 & 14 & NA & \multicolumn{3}{|c|}{1} & \\
\hline Name & 1278 & $720 \mathrm{~J} / \mathrm{K}$ & DBC3 & 5630 & & DBC3 & $605 \mathrm{~K}$ & S1 & $\mathbf{5 2}$ & 53 & NG-S \\
\hline $2,4 \mathrm{D}$ & $.5 \mathrm{mg} / \mathrm{L}$ & $1.5 \mathrm{mg} / \mathrm{L}$ & $1 \mathrm{mg} / \mathrm{L}$ & $1.5 \mathrm{mg} / \mathrm{L}$ & $1.5 \mathrm{mg} / \mathrm{L}$ & $1 \mathrm{mg} / \mathrm{L}$ & $0.8 \mathrm{mg} / \mathrm{L}$ & & & & $1.5 \mathrm{mg} / \mathrm{L}$ \\
\hline 6-benzylaminopurine & $.01 \mathrm{mg} / \mathrm{L}$ & & $.5 \mathrm{mg} / \mathrm{L}$ & & & $.5 \mathrm{mg} / \mathrm{L}$ & & & & & $.5 \mathrm{mg} / \mathrm{L}$ \\
\hline \multicolumn{12}{|l|}{ Absiscic Acid } \\
\hline Agar & & $4 \mathrm{~g} / \mathrm{L}$ & & $8 \mathrm{~g} / \mathrm{L}$ & $6 \mathrm{~g} / \mathrm{L}$ & & & & & & \\
\hline Ascobic Acid & & $10 \mathrm{mg} / \mathrm{L}$ & & & & & & & & & \\
\hline \multicolumn{12}{|l|}{ CA Pantothenate } \\
\hline Carb & $500 \mathrm{mg} / \mathrm{L}$ & $50 \mathrm{mg} / \mathrm{ml}$ & $100 \mathrm{mg} / \mathrm{L}$ & $100 \mathrm{mg} / \mathrm{L}$ & $100 \mathrm{mg} / \mathrm{L}$ & $100 \mathrm{mg} / \mathrm{L}$ & & & & & $100 \mathrm{mg} / \mathrm{L}$ \\
\hline Casamino Acids & $.5 \mathrm{~g} / \mathrm{L}$ & & & & & & & & & & \\
\hline Caseine Hydrolysate & & & $1 \mathrm{~g} / \mathrm{L}$ & & & $1 \mathrm{~g} / \mathrm{L}$ & & & & & $1 \mathrm{~g} / \mathrm{L}$ \\
\hline Cefotaxime & & & & & & & $100 \mathrm{mg} / \mathrm{L}$ & & & & \\
\hline Copper Sulfate & & & $4.9 \mathrm{um}$ & & & $4.9 \mathrm{uM}$ & & & & & $4.9 \mathrm{uM}$ \\
\hline Dicamba & & & & & & & $1.2 \mathrm{mg} / \mathrm{L}$ & $5 \mathrm{mg} / \mathrm{L}$ & $5 \mathrm{mg} / \mathrm{L}$ & $5 \mathrm{mg} / \mathrm{L}$ & \\
\hline \multicolumn{12}{|l|}{ FeEDTA } \\
\hline Gelrite & & & & & & & & $2.4 \mathrm{~g} / \mathrm{L}$ & $2.4 \mathrm{~g} / \mathrm{L}$ & & $3 \mathrm{~g} / \mathrm{L}$ \\
\hline Glucose & & & & & & & $0.6 \mathrm{~g} / \mathrm{L}$ & & & $2.4 \mathrm{~g} / \mathrm{L}$ & \\
\hline \multicolumn{12}{|l|}{ Glycine } \\
\hline L-Proline & $1.38 \mathrm{~g} / \mathrm{L}$ & $.7 \mathrm{~g} / \mathrm{L}$ & $.69 \mathrm{~g} / \mathrm{L}$ & $.69 \mathrm{~g} / \mathrm{L}$ & $.69 \mathrm{~g} / \mathrm{L}$ & $.69 \mathrm{~g} / \mathrm{L}$ & & $1.38 \mathrm{~g} / \mathrm{L}$ & $1.38 \mathrm{~g} / \mathrm{L}$ & & $.7 \mathrm{~g} / \mathrm{L}$ \\
\hline \multicolumn{12}{|l|}{ LS Salt } \\
\hline Maltose & & & $30 \mathrm{~g} / \mathrm{L}$ & & & $30 \mathrm{~g} / \mathrm{L}$ & & & & & \\
\hline MES Salts & & $.5 \mathrm{~g} / \mathrm{L}$ & & $.5 \mathrm{~g} / \mathrm{L}$ & $.5 \mathrm{~g} / \mathrm{L}$ & & & & & & \\
\hline MS Salts & $4.33 \mathrm{~g} / \mathrm{L}$ & $4.3 \mathrm{~g} / \mathrm{L}$ & $4.3 \mathrm{~g} / \mathrm{L}$ & & & $4.3 \mathrm{~g} / \mathrm{L}$ & Yes & $4.3 \mathrm{~g} / \mathrm{L}$ & $4.3 \mathrm{~g} / \mathrm{L}$ & $4.29 \mathrm{~g} / \mathrm{L}$ & $4.3 \mathrm{~g} / \mathrm{L}$ \\
\hline MS Vitamins & Yes & & & & & & Yes & Yes & Yes & Yes & \\
\hline Myo-inositol & & $.1 \mathrm{~g} / \mathrm{L}$ & $.25 \mathrm{~g} / \mathrm{L}$ & & & $.25 \mathrm{~g} / \mathrm{L}$ & & & & & $.25 \mathrm{~g} / \mathrm{L}$ \\
\hline N6 Salt & & & & $4 \mathrm{~g} / \mathrm{L}$ & $4 \mathrm{~g} / \mathrm{L}$ & & Yes & & & & \\
\hline N6 Vitamin & & & & Yes & Yes & & & & & & \\
\hline Nicotinic Acid & & $.5 \mathrm{mg} / \mathrm{L}$ & & & & & & & & & \\
\hline $\mathrm{pH}$ & 5.8 & 5.8 & 5.8 & 5.8 & 5.8 & 5.8 & 5.8 & & & & 5.8 \\
\hline \multicolumn{12}{|l|}{ Phytagar } \\
\hline Phytagel & $3 \mathrm{~g} / \mathrm{L}$ & & $3.5 \mathrm{~g} / \mathrm{L}$ & & & $3.5 \mathrm{~g} / \mathrm{L}$ & & & & & \\
\hline Pyridoxine $\mathrm{HCl}$ & & $.5 \mathrm{mg} / \mathrm{L}$ & & & & & & & & & \\
\hline Selection & $0.1 \mathrm{mM}$ Glyphosate & $\begin{array}{c}50 \mathrm{mg} / \mathrm{L}(2 \mathrm{zk}) \text { then } 100 \mathrm{mg} / \mathrm{L} \\
\text { glufosinate }\end{array}$ & $3 \mathrm{mg} / \mathrm{L}$ bialaphos & $3 \mathrm{mg} / \mathrm{L}$ bialaphos & $3 \mathrm{mg} / \mathrm{L}$ bialaphos & $\begin{array}{l}.25 \mathrm{mM} \text { to } .5 \mathrm{mM} \\
\text { Glyphosate }\end{array}$ & & $7.5 \mathrm{mg} / \mathrm{L}$ & $7.5 \mathrm{mg} / \mathrm{L}$ & $5 \mathrm{mg} / \mathrm{L}$ & $\begin{array}{c}1.5 \mathrm{mg} / \mathrm{L}(2 \mathrm{wk}) \text { then } 3 \mathrm{mg} / \mathrm{L} \\
\text { bialaphos }\end{array}$ \\
\hline Silver Nitrate & $3.4 \mathrm{mg} / \mathrm{L}$ & & & $.85 \mathrm{mg} / \mathrm{L}$ & $.85 \mathrm{mg} / \mathrm{L}$ & & & & & & $1.7 \mathrm{mg} / \mathrm{L}$ \\
\hline Sucrose & $30 \mathrm{~g} / \mathrm{L}$ & $20 \mathrm{~g} / \mathrm{L}$ & & $30 \mathrm{~g} / \mathrm{L}$ & $30 \mathrm{~g} / \mathrm{L}$ & & $20 \mathrm{~g} / \mathrm{L}$ & $5 \mathrm{~g} / \mathrm{L}$ & $10 \mathrm{~g} / \mathrm{L}$ & $20 \mathrm{~g} / \mathrm{L}$ & $30 \mathrm{~g} / \mathrm{L}$ \\
\hline Thiamine & $.5 \mathrm{mg} / \mathrm{L}$ & $10 \mathrm{mg} / \mathrm{L}$ & $20 \mathrm{mg} / \mathrm{L}$ & $.5 \mathrm{mg} / \mathrm{L}$ & $1 \mathrm{mg} / \mathrm{L}$ & $20 \mathrm{mg} / \mathrm{L}$ & & & & & $1.5 \mathrm{mg} / \mathrm{L}$ \\
\hline Ticarcillin & & & & & & & & $200 \mathrm{mg} / \mathrm{L}$ & $200 \mathrm{mg} / \mathrm{L}$ & $200 \mathrm{mg} / \mathrm{L}$ & \\
\hline \multirow{2}{*}{\multicolumn{12}{|c|}{ Vancomycin }} \\
\hline & & & & & & & & & & & \\
\hline \multirow[t]{3}{*}{ Time } & 2-3 wk & $720 \mathrm{~J} 2 \mathrm{wk}-28 \mathrm{C}$ & 2-3 wk Fresh & & 3 wk Fresh & $\begin{array}{l}\text { 3-4 wk Reduced } \\
\text { Glyphosate }\end{array}$ & 2-3 wk Fresh & & & & 2 wk Reduced Bialaphos \\
\hline & & $720 \mathrm{k} 2$ wk Fresh & $6-8$ Total & & & 3-4wk Full Glyphosate & 2-2.5 Month & & & & $\begin{array}{l}\text { At Least } 4 \text { wk Full Selection } \\
\text { Until Fast Growing }\end{array}$ \\
\hline & & Identified by $1 \mathrm{~cm}$ callus & Identified by growth & & & \begin{tabular}{|l|} 
Identified by Growth \\
\end{tabular} & & & & & Change every 2 wk \\
\hline
\end{tabular}

Table 5 Selection Media Continued 


\begin{tabular}{|c|c|c|c|c|c|c|c|c|c|c|}
\hline \multicolumn{11}{|c|}{ Shooting Media } \\
\hline Source & lowa & Japan Tobacco & Japan Tobacco & Japan Tobacco & Missouri & Monsanto & \begin{tabular}{|l|} 
Monsanto \\
\end{tabular} & Monsanto & Monsanto & Monsanto \\
\hline Date & 2002 & $2 / 28 / 96$ & $8 / 12 / 04$ & $3 / 27 / 14$ & 2014 & $6 / 1 / 04$ & \begin{tabular}{|l|}
$8 / 31 / 07$ \\
\end{tabular} & $8 / 31 / 07$ & $3 / 22 / 10$ & $3 / 8 / 13$ \\
\hline Countries Filed & NA & NA & 8 & 5 & NA & 1 & 10 & 10 & 1 & 10 \\
\hline Name & Regeration I & LSZ & & LSZ & PhiE & MSW50 (5 days) & $\mathrm{MS} / 6 \mathrm{BA}$ & 1344 Regeneration & $\mathrm{MS} / 6 \mathrm{BA}$ & Part of Selection \\
\hline \multicolumn{11}{|l|}{$2,4 \mathrm{D}$} \\
\hline 6-benzylaminopurine & & & & & & & \begin{tabular}{|l|}
$3.5 \mathrm{mg} / \mathrm{L}$ \\
\end{tabular} & $3.5 \mathrm{mg} / \mathrm{L}$ & $3.5 \mathrm{mg} / \mathrm{L}$ & \\
\hline \multicolumn{11}{|l|}{ ABA } \\
\hline \multicolumn{11}{|l|}{ ABD } \\
\hline Agar & & $8 \mathrm{~g} / \mathrm{L}$ & $8 \mathrm{~g} / \mathrm{L}$ & $8 \mathrm{~g} / \mathrm{L}$ & & & & & & \\
\hline \multicolumn{11}{|l|}{ Asparagine } \\
\hline Carb & & & & $250 \mathrm{mg} / \mathrm{L}$ & & & & $250 \mathrm{mg} / \mathrm{L}$ & $250 \mathrm{mg} / \mathrm{L}$ & \\
\hline Casamino acids & & & & & & $500 \mathrm{mg} / \mathrm{L}$ & $50 \mathrm{mg} / \mathrm{L}$ & $.5 \mathrm{~g} / \mathrm{L}$ & $50 \mathrm{mg} / \mathrm{L}$ & \\
\hline Cefotaxime & $250 \mathrm{mg} / \mathrm{L}$ & $250 \mathrm{mg} / \mathrm{L}$ & & $100 \mathrm{mg} / \mathrm{L}$ & $250 \mathrm{mg} / \mathrm{L}$ & & & & & \\
\hline Copper Sulfate & & & & $10 \mathrm{um}$ & & & & & & \\
\hline FeEDTA & & & & $1 \mathrm{mM}$ & & & & & & \\
\hline Gelrite & $3 \mathrm{~g} / \mathrm{L}$ & & & & $3 \mathrm{~g} / \mathrm{L}$ & & & & & \\
\hline \multicolumn{11}{|l|}{ Gelzan } \\
\hline \multicolumn{11}{|l|}{ Glucose } \\
\hline Glycine & & & & & $2 \mathrm{mg} / \mathrm{L}$ & $2 \mathrm{mg} / \mathrm{L}$ & & & & \\
\hline \multicolumn{11}{|l|}{ IAA } \\
\hline \multicolumn{11}{|l|}{ Kinetin } \\
\hline L-Proline & & $.7 \mathrm{~g} / \mathrm{L}$ & $700 \mathrm{mg} / \mathrm{L}$ & & & $1.38 \mathrm{~g} / \mathrm{L}$ & $1.36 \mathrm{~g} / \mathrm{L}$ & $1.38 \mathrm{~g} / \mathrm{L}$ & $1.36 \mathrm{~g} / \mathrm{L}$ & \\
\hline LS Salt & & Yes & & Yes & & & & & & \\
\hline \multicolumn{11}{|l|}{ Maltose } \\
\hline MES Salts & & $.5 \mathrm{~g} / \mathrm{L}$ & $500 \mathrm{mg} / \mathrm{L}$ & $500 \mathrm{mg} / \mathrm{L}$ & & & & & & \\
\hline MS FROMM Vitamins & & & & & & & & Yes & & \\
\hline MS Salt & Yes & & & & $4.3 \mathrm{~g} / \mathrm{L}$ & $4.4 \mathrm{~g} / \mathrm{L}$ & $4.4 \mathrm{~g} / \mathrm{L}$ & $4.33 \mathrm{~g} / \mathrm{L}$ & $4.4 \mathrm{~g} / \mathrm{L}$ & \\
\hline MS Vitamin & Yes & & & & Yes & & & Yes & & \\
\hline \multicolumn{11}{|l|}{ MS FROMM Vitamins } \\
\hline Myo-inositol & $.1 \mathrm{~g} / \mathrm{L}$ & $.1 \mathrm{~g} / \mathrm{L}$ & $.1 \mathrm{~g} / \mathrm{L}$ & $.1 \mathrm{~g} / \mathrm{L}$ & $.1 \mathrm{~g} / \mathrm{L}$ & $100 \mathrm{mg} / \mathrm{L}$ & & & & \\
\hline Nicotinic Acid & & $.5 \mathrm{mg} / \mathrm{L}$ & $.5 \mathrm{mg} / \mathrm{L}$ & $.5 \mathrm{mg} / \mathrm{L}$ & $.5 \mathrm{mg} / \mathrm{L}$ & $.5 \mathrm{mg} / \mathrm{L}$ & $1.3 \mathrm{mg} / \mathrm{L}$ & & $1.3 \mathrm{mg} / \mathrm{L}$ & \\
\hline pH & & 5.8 & 5.8 & 5.8 & 5.6 & & & 5.8 & & \\
\hline Phytagel & & & & & & & & $3 \mathrm{~g} / \mathrm{L}$ & & \\
\hline Pyridoxine & & $.5 \mathrm{mg} / \mathrm{L}$ & $.5 \mathrm{mg} / \mathrm{L}$ & $.5 \mathrm{mg} / \mathrm{L}$ & $.5 \mathrm{mg} / \mathrm{L}$ & $.5 \mathrm{mg} / \mathrm{L}$ & $.25 \mathrm{mg} / \mathrm{L}$ & & $.25 \mathrm{mg} / \mathrm{L}$ & \\
\hline Selection & $3 \mathrm{mg} / \mathrm{L}$ Bialaphos & $5 \mathrm{mg} / \mathrm{L}$ Bialaphos & & $5 \mathrm{mg} / \mathrm{L}$ Bialaphos & $3 \mathrm{mg} / \mathrm{L}$ Bialaphos & & & & & \\
\hline Sucrose & $60 \mathrm{~g} / \mathrm{L}$ & $20 \mathrm{~g} / \mathrm{L}$ & $20 \mathrm{~g} / \mathrm{L}$ & $20 \mathrm{~g} / \mathrm{L}$ & $60 \mathrm{~g} / \mathrm{L}$ & $30 \mathrm{~g} / \mathrm{L}$ & $30 \mathrm{~g} / \mathrm{L}$ & $30 \mathrm{~g} / \mathrm{L}$ & $30 \mathrm{~g} / \mathrm{L}$ & \\
\hline Thiamine HCL & & $1 \mathrm{mg} / \mathrm{L}$ & $1 \mathrm{mg} / \mathrm{L}$ & $1 \mathrm{mg} / \mathrm{L}$ & $.1 \mathrm{mg} / \mathrm{L}$ & & $.25 \mathrm{mg} / \mathrm{L}$ & & $.25 \mathrm{mg} / \mathrm{L}$ & \\
\hline \multicolumn{11}{|l|}{ Thidiazuron } \\
\hline \multicolumn{11}{|l|}{ Ticarcillin } \\
\hline Zeatin & & $5 \mathrm{mg} / \mathrm{L}$ & $5 \mathrm{mg} / \mathrm{L}$ & $5 \mathrm{mg} / \mathrm{L}$ & & & & & & \\
\hline \multirow[t]{2}{*}{ Time } & $3 \mathrm{wk}$ & & $3 \mathrm{wk}$ & $14 \mathrm{~d}$ in light & $2-3 \mathrm{wk}$ & $5 d$ & $3 \mathrm{wk}$ & $3 d$ & 5-7d 27C Light/Dark & Skip \\
\hline & & & & & & & & & 2 wk Trays & \\
\hline
\end{tabular}

Table 6 Shooting Media 


\begin{tabular}{|c|c|c|c|c|c|c|c|c|c|c|}
\hline \multicolumn{11}{|c|}{ Shooting Media } \\
\hline Source & \multicolumn{2}{|c|}{ Monsanto } & Pioneer & Pioneer & Pioneer & Pioneer & Pioneer & Pioneer & Syngenta & Consensus \\
\hline Date & \multicolumn{2}{|c|}{$7 / 18 / 13$} & $1 / 21 / 09$ & $1 / 26 / 10$ & $1 / 22 / 10$ & $12 / 30 / 10$ & $3 / 13 / 13$ & $9 / 6 / 16$ & $4 / 23 / 15$ & \\
\hline Countries Filed & \multicolumn{2}{|c|}{10} & 4 & 1 & 1 & 7 & 14 & NA & 1 & \\
\hline Name & 1073 & 1071 & 289J & PhiRF & 289B & Regeneration & PhiRF & Shoot Formation & Regeneration & NG-SH \\
\hline \multicolumn{11}{|l|}{$2,4 \mathrm{D}$} \\
\hline 6-benzylaminopurine & $3.5 \mathrm{mg} / \mathrm{L}$ & & & & & & & $1 \mathrm{mg} / \mathrm{L}$ & & \\
\hline ABA & & & & $26.4 \mathrm{ug} / \mathrm{L}$ & $.1 \mathrm{uM}$ & $.1 \mathrm{uM}$ & $26.4 \mathrm{ug} / \mathrm{L}$ & & & $26.4 \mathrm{ug} / \mathrm{L}$ \\
\hline ABD & & & $.1 \mathrm{uM}$ & & & & & & & \\
\hline Agar & & & $4 \mathrm{~g} / \mathrm{L}$ & $8 \mathrm{~g} / \mathrm{L}$ & & & $8 \mathrm{~g} / \mathrm{L}$ & & & \\
\hline Asparagine & & $.15 \mathrm{~g} / \mathrm{L}$ & & & & & & & & \\
\hline Carb & $250 \mathrm{mg} / \mathrm{L}$ & $250 \mathrm{mg} / \mathrm{L}$ & $100 \mathrm{mg} / \mathrm{L}$ & $100 \mathrm{mg} / \mathrm{L}$ & $100 \mathrm{mg} / \mathrm{L}$ & $100 \mathrm{mg} / \mathrm{L}$ & & $100 \mathrm{mg} / \mathrm{L}$ & & $100 \mathrm{mg} / \mathrm{L}$ \\
\hline Casamino acids & & $.5 \mathrm{~g} / \mathrm{L}$ & & & & & & & & \\
\hline Cefotaxime & & & & & & & $100 \mathrm{mg} / \mathrm{L}$ & & & \\
\hline Copper Sulfate & & & & $.4 \mathrm{uM}$ & & & $.4 \mathrm{uM}$ & & & $.4 \mathrm{uM}$ \\
\hline \multicolumn{11}{|l|}{ FeEDTA } \\
\hline Gelrite & & & & & $3 \mathrm{~g} / \mathrm{L}$ & $3 \mathrm{~g} / \mathrm{L}$ & & & & $3 \mathrm{~g} / \mathrm{L}$ \\
\hline Gelzan & & & & & & & & & $3 \mathrm{~g} / \mathrm{L}$ & \\
\hline Glucose & & $10 \mathrm{~g} / \mathrm{L}$ & & & & & & & & \\
\hline Glycine & & & & $2 \mathrm{mg} / \mathrm{L}$ & $2 \mathrm{mg} / \mathrm{L}$ & & $2 \mathrm{mg} / \mathrm{L}$ & & & $2 \mathrm{mg} / \mathrm{L}$ \\
\hline IAA & & & $1 \mathrm{mg} / \mathrm{L}$ & $1 \mathrm{mg} / \mathrm{L}$ & $1 \mathrm{mg} / \mathrm{L}$ & $1 \mathrm{mg} / \mathrm{L}$ & $1 \mathrm{mg} / \mathrm{L}$ & & $.25 \mathrm{mg} / \mathrm{L}$ & $1 \mathrm{mg} / \mathrm{L}$ \\
\hline Kinetin & & & & & & & & & $1 \mathrm{mg} / \mathrm{L}$ & \\
\hline L-Proline & & & $.7 \mathrm{~g} / \mathrm{L}$ & & & & & & & \\
\hline \multicolumn{11}{|l|}{ LS Salt } \\
\hline Maltose & & $20 \mathrm{~g} / \mathrm{L}$ & & & & & & & & \\
\hline \multicolumn{11}{|l|}{ MES Salts } \\
\hline \multicolumn{11}{|l|}{ MS FROMM Vitamins } \\
\hline MS Salt & $4.33 \mathrm{~g} / \mathrm{L}$ & $4.33 \mathrm{~g} / \mathrm{L}$ & $4.3 \mathrm{~g} / \mathrm{L}$ & $4.3 \mathrm{~g} / \mathrm{L}$ & $4.3 \mathrm{~g} / \mathrm{L}$ & $4.3 \mathrm{~g} / \mathrm{L}$ & $4.3 \mathrm{~g} / \mathrm{L}$ & Yes & $4.3 \mathrm{~g} / \mathrm{L}$ & $4.3 \mathrm{~g} / \mathrm{L}$ \\
\hline MS Vitamin & & & Yes & & & Yes & & & & \\
\hline MS FROMM Vitamins & Yes & Yes & & & & & & & & \\
\hline Myo-inositol & & $.1 \mathrm{~g} / \mathrm{L}$ & $1 \mathrm{~g} / \mathrm{L}$ & $.1 \mathrm{~g} / \mathrm{L}$ & $1 \mathrm{~g} / \mathrm{L}$ & $100 \mathrm{mg} / \mathrm{L}$ & $.1 \mathrm{~g} / \mathrm{L}$ & & & $.1 \mathrm{~g} / \mathrm{L}$ \\
\hline Nicotinic Acid & & & & $.5 \mathrm{mg} / \mathrm{L}$ & $.5 \mathrm{mg} / \mathrm{L}$ & & $.5 \mathrm{mg} / \mathrm{L}$ & & & $.5 \mathrm{mg} / \mathrm{L}$ \\
\hline $\mathrm{pH}$ & 5.8 & 5.8 & 5.6 & 5.6 & 5.6 & 5.6 & 5.6 & 5.8 & & 5.6 \\
\hline Phytagel & $3 \mathrm{~g} / \mathrm{L}$ & $3 \mathrm{~g} / \mathrm{L}$ & & & & & & & & \\
\hline Pyridoxine & & & & $.5 \mathrm{mg} / \mathrm{L}$ & $.5 \mathrm{mg} / \mathrm{L}$ & & $.5 \mathrm{mg} / \mathrm{L}$ & & & $.5 \mathrm{mg} / \mathrm{L}$ \\
\hline Selection & $.1 \mathrm{mM}$ Glyphosate & $.1 \mathrm{mM}$ Glyphosate & $5 \mathrm{mg} / \mathrm{Lg}$ glufosinate & $3 \mathrm{mg} / \mathrm{L}$ Bialaphos & $1.5 \mathrm{mg} / \mathrm{L} \mathrm{Bialaphos}$ & $1.5 \mathrm{mg} / \mathrm{L}$ Bialaphos & Glyphosate & & & $1.5 \mathrm{mg} / \mathrm{L}$ Bialaphos \\
\hline Sucrose & $30 \mathrm{~g} / \mathrm{L}$ & & $60 \mathrm{~g} / \mathrm{L}$ & $60 \mathrm{~g} / \mathrm{L}$ & $60 \mathrm{~g} / \mathrm{L}$ & $60 \mathrm{~g} / \mathrm{L}$ & $60 \mathrm{~g} / \mathrm{L}$ & $60 \mathrm{~g} / \mathrm{L}$ & $20 \mathrm{~g} / \mathrm{L}$ & $60 \mathrm{~g} / \mathrm{L}$ \\
\hline Thiamine HCL & & & & $.1 \mathrm{mg} / \mathrm{L}$ & $.1 \mathrm{mg} / \mathrm{L}$ & & $.1 \mathrm{mg} / \mathrm{L}$ & & & $.1 \mathrm{mg} / \mathrm{L}$ \\
\hline Thidiazuron & & & $.1 \mathrm{mg} / \mathrm{L}$ & $.1 \mathrm{mg} / \mathrm{L}$ & & & $.1 \mathrm{mg} / \mathrm{L}$ & $0.1 \mathrm{mg} / \mathrm{L}$ & & $.1 \mathrm{mg} / \mathrm{L}$ \\
\hline Ticarcillin & & & & & & & & & $200 \mathrm{mg} / \mathrm{L}$ & \\
\hline Zeatin & & & $.5 \mathrm{mg} / \mathrm{L}$ & $.5 \mathrm{mg} / \mathrm{L}$ & $.5 \mathrm{mg} / \mathrm{L}$ & $.5 \mathrm{mg} / \mathrm{L}$ & $.5 \mathrm{mg} / \mathrm{L}$ & $0.5 \mathrm{mg} / \mathrm{L}$ & & $.5 \mathrm{mg} / \mathrm{L}$ \\
\hline Time & 5-7 Days $27 \mathrm{C}$ & 2 wks & & \begin{tabular}{|l|}
$10-18$ days $28 \mathrm{C}$ \\
\end{tabular} & & $2 w k-28 C$ & $2-3 \mathrm{wk}$ & $2-3 \mathrm{wk}$ & & Until Shoots \\
\hline
\end{tabular}

Table 6 Shooting Media Continued 


\begin{tabular}{|c|c|c|c|c|c|c|c|c|c|c|}
\hline \multicolumn{11}{|c|}{ Rooting Media } \\
\hline Source & lowa State & Japan Tobacco & Japan Tobacco & Japan Tobacco & Missouri & Monsanto & Monsanto & Monsanto & Monsanto & Monsanto \\
\hline Year Published & 2002 & 2/28/96 & $8 / 12 / 04$ & $3 / 27 / 14$ & 2004 & $6 / 1 / 04$ & $8 / 31 / 07$ & $3 / 22 / 10$ & $3 / 8 / 13$ & $7 / 18 / 13$ \\
\hline Countries Filed & NA & NA & 8 & 5 & NA & 1 & 10 & 1 & 10 & 10 \\
\hline Name & Regeneration II & $1 / 2$ LSF & N/A & LSF & PhiF & MSOD & 1607 Growth & MSOD & Part of Selection & 1084 \\
\hline \multicolumn{11}{|l|}{$2,4 \mathrm{D}$} \\
\hline 6-benzylaminopurine & & & & & & & $.02 \mathrm{mM}$ & & & \\
\hline Agar & & $8 \mathrm{~g} / \mathrm{L}$ & & & & & & & & \\
\hline Asparagine & & & & & & $150 \mathrm{mg} / \mathrm{L}$ & & $150 \mathrm{mg} / \mathrm{L}$ & & \\
\hline \multicolumn{11}{|l|}{ Basal Salts } \\
\hline \multicolumn{11}{|l|}{ Benomyl } \\
\hline Ca Pantothenate & & & & & & $.25 \mathrm{mg} / \mathrm{L}$ & & $.25 \mathrm{mg} / \mathrm{L}$ & & \\
\hline Carb & & & & & & & $100 \mathrm{mg} / \mathrm{L}$ & & & \\
\hline Cefotaxime & & $250 \mathrm{mg} / \mathrm{L}$ & & & & & & & & \\
\hline FeEDTA & & & & $1 \mathrm{mM}$ & & & & & & \\
\hline Gelrite & $3 \mathrm{~g} / \mathrm{L}$ & & & & $3 \mathrm{~g} / \mathrm{L}$ & & & & & \\
\hline \multicolumn{11}{|l|}{ Gelzan } \\
\hline Glucose & & & & & & $10 \mathrm{~g} / \mathrm{L}$ & & $10 \mathrm{~g} / \mathrm{L}$ & & \\
\hline Glycine & & & & & $2 \mathrm{mg} / \mathrm{L}$ & & & & & \\
\hline \multicolumn{11}{|l|}{ IAA } \\
\hline IBA & & & & $.2 \mathrm{mg} / \mathrm{L}$ & & & & & & $.75 \mathrm{mg} / \mathrm{L}$ \\
\hline LS Salt & & Yes & & Yes & & & & & & \\
\hline Maltose & & & & & & $20 \mathrm{~g} / \mathrm{L}$ & & $20 \mathrm{~g} / \mathrm{L}$ & & \\
\hline MES Salts & & $.5 \mathrm{~g} / \mathrm{L}$ & & $.5 \mathrm{~g} / \mathrm{L}$ & & & & & & \\
\hline MS Salt & Yes & & & & $2.9 \mathrm{~g} / \mathrm{L}$ & $4.4 \mathrm{~g} / \mathrm{L}$ & $4.3 \mathrm{~g} / \mathrm{L}$ & $4.4 \mathrm{~g} / \mathrm{L}$ & & $2.165 \mathrm{~g} / \mathrm{L}$ \\
\hline MS Vitamin & Yes & & & & & & Yes & & & \\
\hline Myo-Inositol & $100 \mathrm{mg} / \mathrm{L}$ & $100 \mathrm{mg} / \mathrm{L}$ & & $.1 \mathrm{~g} / \mathrm{L}$ & . $1 \mathrm{~g} / \mathrm{L}$ & $100 \mathrm{mg} / \mathrm{L}$ & & $100 \mathrm{mg} / \mathrm{L}$ & & \\
\hline NAA & & & & & & & & & & $.5 \mathrm{mg} / \mathrm{L}$ \\
\hline Nicotinic Acid & & $.5 \mathrm{mg} / \mathrm{L}$ & & $.5 \mathrm{mg} / \mathrm{L}$ & $.5 \mathrm{mg} / \mathrm{L}$ & $1.3 \mathrm{mg} / \mathrm{L}$ & & $1.3 \mathrm{mg} / \mathrm{L}$ & & \\
\hline pH & & 5.8 & & & 5.6 & & 5.8 & & & 5.8 \\
\hline Phytagar & & & & & & & $6 \mathrm{~g} / \mathrm{L}$ & & & \\
\hline Phytagel & & & & & & & & & & $3 \mathrm{~g} / \mathrm{L}$ \\
\hline Pyridoxine & & $.5 \mathrm{mg} / \mathrm{L}$ & & $.5 \mathrm{mg} / \mathrm{L}$ & $.5 \mathrm{mg} / \mathrm{L}$ & $.25 \mathrm{mg} / \mathrm{L}$ & & $.25 \mathrm{mg} / \mathrm{L}$ & & \\
\hline Selection & & & & & & & & & & $.1 \mathrm{mM}$ Glyphosate \\
\hline Sucrose & $30 \mathrm{~g} / \mathrm{L}$ & $20 \mathrm{~g} / \mathrm{L}$ & & $15 \mathrm{~g} / \mathrm{L}$ & $30 \mathrm{~g} / \mathrm{L}$ & & $60 \mathrm{~g} / \mathrm{L}$ & & & $20 \mathrm{~g} / \mathrm{L}$ \\
\hline \multicolumn{11}{|l|}{ Ticarcillin } \\
\hline Thiamine HCL & & $1 \mathrm{mg} / \mathrm{L}$ & & $1 \mathrm{mg} / \mathrm{L}$ & $.1 \mathrm{mg} / \mathrm{L}$ & $.25 \mathrm{mg} / \mathrm{L}$ & & $.25 \mathrm{mg} / \mathrm{L}$ & & \\
\hline \multicolumn{11}{|l|}{ Vitamin Mix } \\
\hline Time & Until Plants & $2 \mathrm{wk}$ & & Until Plants in test tubes & Until Plants & $3 \mathrm{wk}$ & & $10 \mathrm{~d}$ & Skip & \\
\hline
\end{tabular}

Table 7 Rooting Media 


\begin{tabular}{|c|c|c|c|c|c|c|c|c|c|}
\hline \multicolumn{10}{|c|}{ Rooting Media } \\
\hline Source & Pioneer & Pioneer & Pioneer & Pioneer & Pioneer & Pioneer & Pioneer & Syngenta & Consensus \\
\hline Year Published & $1 / 21 / 09$ & $1 / 26 / 10$ & $1 / 22 / 10$ & $12 / 30 / 10$ & $3 / 13 / 13$ & $9 / 6 / 16$ & $9 / 6 / 16$ & $4 / 23 / 16$ & \\
\hline Countries Filed & 4 & 1 & 1 & 7 & 14 & NA & NA & 1 & \\
\hline Name & With Shooting & PhiRR & $271 C$ & $?$ & MSB? & Root Development & Seed Germination & Rooting & NG-RG \\
\hline \multicolumn{10}{|l|}{$2,4 D$} \\
\hline \multicolumn{10}{|c|}{ 6-benzylaminopurine } \\
\hline Agar & & $6 \mathrm{~g} / \mathrm{L}$ & & & & & & & \\
\hline \multicolumn{10}{|l|}{ Asparagine } \\
\hline Basal Salts & & & & & & & & $3.2 \mathrm{~g} / \mathrm{L}$ & \\
\hline Benomyl & & & & & & & $100 \mathrm{mg} / \mathrm{L}$ & & \\
\hline \multicolumn{10}{|l|}{ Ca Pantothenate } \\
\hline \multicolumn{10}{|l|}{ Carb } \\
\hline \multicolumn{10}{|l|}{ Cefotaxime } \\
\hline \multicolumn{10}{|l|}{ FeEDTA } \\
\hline Gelrite & & & $1.5 \mathrm{~g} / \mathrm{L}$ & $1.5 \mathrm{~g} / \mathrm{L}$ & & & & & $3 \mathrm{~g} / \mathrm{L}$ \\
\hline Gelzan & & & & & & & & $2.4 \mathrm{~g} / \mathrm{L}$ & \\
\hline \multicolumn{10}{|l|}{ Glucose } \\
\hline Glycine & & & $2 \mathrm{mg} / \mathrm{L}$ & & & & & & $2 \mathrm{mg} / \mathrm{L}$ \\
\hline \multicolumn{10}{|l|}{ IAA } \\
\hline \multicolumn{10}{|l|}{ IBA } \\
\hline \multicolumn{10}{|l|}{ LS Salt } \\
\hline \multicolumn{10}{|l|}{ Maltose } \\
\hline \multicolumn{10}{|l|}{ MES Salts } \\
\hline MS Salt & & $4.3 \mathrm{~g} / \mathrm{L}$ & $4.3 \mathrm{~g} / \mathrm{L}$ & $4.3 \mathrm{~g} / \mathrm{L}$ & & Yes & Yes & & $4.3 \mathrm{~g} / \mathrm{L}$ \\
\hline MS Vitamin & & Yes & & Yes & & & & & \\
\hline Myo-Inositol & & & $.1 \mathrm{~g} / \mathrm{L}$ & $100 \mathrm{mg} / \mathrm{L}$ & & & & & $.1 \mathrm{~g} / \mathrm{L}$ \\
\hline NAA & & & & & & & & $0.5 \mathrm{mg} / \mathrm{L}$ & \\
\hline Nicotinic Acid & & & $.5 \mathrm{mg} / \mathrm{L}$ & & & & & & $.5 \mathrm{mg} / \mathrm{L}$ \\
\hline $\mathrm{pH}$ & & 5.6 & 5.6 & 5.6 & & 5.8 & 5.8 & & 5.6 \\
\hline \multicolumn{10}{|l|}{ Phytagar } \\
\hline \multicolumn{10}{|l|}{ Phytagel } \\
\hline Pyridoxine & & & $.5 \mathrm{mg} / \mathrm{L}$ & & & & & & $.5 \mathrm{mg} / \mathrm{L}$ \\
\hline Selection & & & $3 \mathrm{mg} / \mathrm{L}$ Bialaphos & & & & & & \\
\hline Sucrose & & $40 \mathrm{~g} / \mathrm{L}$ & $40 \mathrm{~g} / \mathrm{L}$ & $40 \mathrm{~g} / \mathrm{L}$ & & $40 \mathrm{~g} / \mathrm{L}$ & $40 \mathrm{~g} / \mathrm{L}$ & $30 \mathrm{~g} / \mathrm{L}$ & $35 \mathrm{~g} / \mathrm{L}$ \\
\hline Ticarcillin & & & & & & & & $200 \mathrm{mg} / \mathrm{L}$ & \\
\hline Thiamine HCL & & & $.1 \mathrm{mg} / \mathrm{L}$ & & & & & & $.1 \mathrm{mg} / \mathrm{L}$ \\
\hline Vitamin Mix & & & & & & & & Yes & \\
\hline Time & & 7-10 d 28C Light & & $1 \mathrm{wk}-28 \mathrm{C}$ Light & 7-10 d 28C Light & & & & \\
\hline
\end{tabular}

Table 7 Rooting Media Continued 


\section{Patent Review and Formation of Consensus Protocol}

To form a consensus protocol, it was necessary to examine each step in the transformation process individually. Each step was examined assuming that the patented process was likely not developed for $\mathrm{Hi}-\mathrm{II}$, and that minor details may not have been given. Additionally, changes that were extremely outside of the currently used process were not considered because sufficient detail was not usually given. In many cases, inclusion of an ingredient was noted, but the precise amount not given. This was often the case for vitamin mixtures, where MS or N6 vitamins were listed, but the exact amounts of each were not stated. This is notable as when vitamin mixtures were listed; they often varied between protocols, despite being given the same nomenclature. While individual components were altered throughout the process, there were two main alterations of note. First, despite academic protocols being based around N6 salts and vitamins until the shooting step when MS salts and vitamins are used, reviewing the patent literature suggests that it is best to use MS media throughout. Secondly, the patents that were filed internationally, and thus probably of the most importance, included some sort of growth regulator to accelerate callus growth.

The inoculation step (table 2) was fairly consistent across the protocols, though full details were often omitted. The process of preparing Agrobacterium cultures was noted to be a quite long and complex process in the Monsanto 
patents. Cultures are noted as being grown supplemented with acetosyringone over 24 hours before the initial inoculation, as compared to a few hours for standard protocols. The major differences in media composition between the currently used academic protocols, and those in table 2 was the switch from an N6 salt base to MS salts. This change was consistent across all steps in the process. Additionally, the inclusion of casamino acids and myo-inositol was fairly consistent across the protocols.

The first large changes in media composition were seen in the cocultivation step (table 3). The widely filed patents of both Monsanto and Pioneer began including either 6-benzylaminopurine (BAP) or ascorbic acid, respectively. While there are various reasons given in the patent explanations, the most consistent explanation is that the embryos are signaled to start shoot development from the very beginning of the transformation process. This is contrary to the current academic strategy where shoot formation is passive during the selection process. In that strategy, callus is not typically moved to shoot formation media until embryoids can be observed either under the microscope or by eye, a process that does not typically occur until callus has been under full selection for many weeks. By including signaling chemicals early in the process, waiting for embryoid formation is no longer necessary later in the process. The second change that occurs from this step forward is the inclusion of copper sulfate in the media. The addition of copper sulfate was mentioned by both Japan Tobacco Company and Pioneer, and was thought to improve callus formation. 
The length of time embryos were exposed to Agrobacterium during cocultivation was not consistent. While most protocols, including those used in academic labs, rely on a 3-4 day exposure time, Monsanto patents suggest an exposure of up to 2 weeks. However, unlike traditional cocultivation media, the media used in these patents also includes a low level of carbenicillin, presumably to prevent overgrowth. As mentioned before, however, the preparation of Agrobacterium for these protocols is different as well. Because the consensus protocol maintained the more traditional Agrobacterium preparation strategy, a standard 3 day exposure was also chosen.

The resting media step (table 4), which is usually 5-7 days in traditional protocols, was often skipped in patented protocols. The resting step is designed to allow embryos to fully differentiate into callus, and to ensure that transformed cells are fully producing resistance protein. It is of note that the internationally filed patents did not skip this step, suggesting that there could be a loss of efficiency when performed on a large scale. It should be reiterated that many patents were designed for faster-growing lines developed in industry breeding programs rather than $\mathrm{Hi}-\mathrm{II}$. A healthier and faster-growing embryo would begin differentiation into callus at a faster rate, and thus may not require a resting step before selection. In practice, it is generally found that once callus is moved to selection media it is slower growing, and the differentiation process may appear to stop completely until resistant cells emerge. However, if callus is allowed to become too large before being moved to selection media, it is often difficult to locate resistant cells. Callus cells not in contact with the media will not be 
exposed to selection, and thus will persist longer and can become false-positives later in the process. As a result, the resting step is generally carried out until friable callus is observed, when the callus is divided onto a selection plate with as many cells exposed to selection as possible. Skipping the resting process, while possibly saving a week during the transformation process, could result in either no-friable callus, or no resistant cells. As no direct explanation of appearance of the callus before being moved to selection could be found in patented literature, a short resting period was maintained in the consensus protocol. The strategy of including growth hormones was continued in the resting step. Interestingly, this strategy was employed by highly-patented protocols from both Monsanto and Pioneer.

The resting stage also serves to eliminate Agrobacterium from the developing callus. This is important as prolonged exposure of embryos or callus to Agrobacterium will often lead to weak or dead embryos. The choice of antibiotic has, in standard protocols, been cefotaxime for this purpose. While effective, it is often used at $250 \mathrm{mg} / \mathrm{L}$ to effectively eliminate Agrobacterium growth. Interestingly, carbenicillin is used in many patented protocols (table 4). Many, Monsanto protocols utilize a high concentration $(500 \mathrm{mg} / \mathrm{L})$ during the resting stage, and then switch to a lower dose for the rest of the procedure. The choice of antibiotic, and corresponding concentration, will most likely vary depending on Agrobacterium strain and growth conditions. Additionally, it is possible that a lower concentration of antibiotic can influence callus growth 
conditions. For this reason, use of carbenicillin was tested as part of the consensus protocol.

The largest variance in methodology and media composition involved the selection step of the process (table 5). As most of the time during the transformation process is spent on this step, this was also thought to have the most potential for improvement. In the standard protocols of lowa State and the University of Missouri, using bialaphos selection, callus is first transferred to a weak selection $(1.5 \mathrm{mg} / \mathrm{L})$ for two weeks before being transferred to full selection (3 mg/L) until embryoids form. The weaker selection step is thought to prevent weaker events from being lost before there can be a sufficient buildup of resistance protein. Interestingly, in many cases, patented protocols maintained this strategy of changing resistance solutions. This strategy was maintained in the consensus protocol.

Many of the previously discussed additives continue to be employed in the selection step. Both Monsanto and Pioneer continue to include BAP; however Pioneer includes it at a much higher concentration. Additionally, copper continued to be included in the media in Pioneer patents, and was thus maintained in the consensus protocol. Myo-inositol, which has been included throughout the process, continues to be consistently added by the various companies.

The largest change in the selection step, and indeed the entire protocol, is the propagation of the callus. In the standard protocols, callus is continuously maintained on selection media until it is friable, well growing, and small 
embryoids can be observed. Usually, this means that callus is transferred to new selection plates every two weeks, with care taken to flatten the callus pieces to ensure full contact with the selection media. Once well-growing callus has been maintained into large sectors it is continued until it gives the appearance of differentiation, in the form of embryoids. The callus is then switched to shooting media to continue the transformation process. In the patented protocols, selection is carried out for much less time. For the most part, the strategy seems to be to force shoot development as fast as possible to accelerate the process. Some protocols even expose selection media to light/dark cycle conditions. It should be noted that most protocols are selective with exact details on when callus is moved to shooting media, and only give rough timelines for the process. However, there is enough information given amongst the various patents to decipher the basic strategies. In general, patented protocols no-longer wait for embryoid development, but transfer to shooting medium once well-growing callus is observed, or callus has obtained a certain size. Some patents mentioned switching to shooting media after a set time period as well. In either case, the strategy for selection has favored an accelerated pace.

While accelerating the transformation process is the ultimate goal, it is worth mentioning that a shorter selection step will most likely result in a larger amount of false-positive plants making it through the process. Continued exposure to selection naturally reduces the amount of callus that does not contain the resistance gene surviving. At the same time, however, the shooting medium also contains selection, so transferred callus will continue to be 
exposed. While false-positives ultimately result in more plants that need to be screened following regeneration, a simple PCR screen can quickly determine if a regenerated plant is transgenic. A shorter selection time also means that a given callus will usually be fairly small, and it may not be possible to recover multiple plants from a single event. Despite the possible negative consequences of a shortened selection scheme, it was ultimately determined that the amount of time and resources saved would be worth the trade-off. Due to the varied strategies employed to determine when to switch to shooting media, however, experimentation was required to determine the exact amount of time to be used with $\mathrm{Hi}-\mathrm{Il}$ and the consensus protocol.

The shooting stage (table 6) was when traditional protocols switched to MS media; however, there are still many differences between these protocols and those patented. The most obvious change was the inclusion of various growth hormones to trigger shoot formation. While previous stages have included BAP thus far, protocols from Pioneer suggest that utilizing a mix of abscisic acid, indole-3-acetic acid, thidiazuron, and zeatin can more rapidly cause shoot formation. Japan Tobacco and Syngenta also used hormones in their media formulations, though to a lesser extent. Monsanto, instead of switching to different hormones, simply increased the concentration of BAP in their shooting media. As with selection media, the inclusion of hormones is designed to accelerate the transformation process, and indeed can lead to the same issues. However, as callus has already been through the selection stage it is advantageous to quickly form plantlets for screening. Additionally, many 
protocols list carrying out the shooting phase under light/dark cycles as it may rapidly induce shoot growth. This option was not considered simply because of lack of sterile facilities with the space required to place dozens of plates under those light conditions. While timing is included in table 6, the consensus protocol, in keeping with the patented protocols, relies on moving callus to rooting media once shoots are observed on the plates instead of a set time period.

The final stage, rooting (table 7), was extremely similar across all protocols, including the standard ones used in academic labs. Not noted on table 7 was the switch from Phytatrays (Sigma-Aldrich P1552) traditionally used for regeneration, to capped culture tubes. While the result is much the same for each method, culture tubes allow for each plant to be maintained separately, allowing for differences in growth time. As with shooting media, there is no set time period for rooting media. Plantlets are moved into soil in a growth chamber once they have reached the top of the culture tube, and roots have mostly filled the bottom.

\section{Materials and Methods}

\section{Patent Review}

Patent review was carried out through the search engine Google Patents (patents.google.com). Search terms such as "maize transformation" were used and relevant patents entered into table 1. Care was taken to find patents from Monsanto Inc., Pioneer Hi-Bred, and Syngenta. As Monsanto and Pioneer published the greatest amount on the subject, effort was made to find patents 
from various time points for comparison. Once relevant patents were obtained, those that published solutions and techniques were used to create tables 2 through 7. To determine importance of the patents to the filing company, patent numbers were entered into espacenet.com to find the patent family. The number of countries in which a patent was filed was then recorded into the tables as well.

\section{Consensus Protocol Development}

Once the tables were complete, a comparison was made between different protocols. Components that were utilized in a majority of the protocols were found, with emphasis placed on components utilized in protocols filed multiple times. These components were entered into the last column of the tables to form the solutions for the final consensus protocol (table 8). The same technique was used to find a consensus for length of time callus is rested on each solution.

Stock Solutions and Chemicals

\begin{tabular}{|l|l|l|}
\hline Chemical & Source & Product Number \\
\hline $\begin{array}{l}\text { Murashige and Skoog Basal Salt Mixture } \\
\text { (MS Salts) }\end{array}$ & Sigma-Aldrich & M5519 \\
\hline Sucrose & Sigma-Aldrich & S7903 \\
\hline Glucose & Sigma-Aldrich & G7021 \\
\hline L-Proline & Sigma-Aldrich & P0380 \\
\hline
\end{tabular}




\begin{tabular}{|l|l|l|}
\hline MES Salts & Fisher Scientific & ICN19483580 \\
\hline Casamino Acids & Fisher Scientific & BP1424 \\
\hline Casein Hydrolysate & Sigma-Aldrich & 22090 \\
\hline 2,4 - dichlorophenoxyacetic acid (2,4-D) & Sigma-Aldrich & D7299 \\
\hline Copper Sulfate Pentahydrate & Fisher Scientific & Bp346 \\
\hline Gelrite & Sigma-Aldrich & G1910 \\
\hline Agar & Sigma-Aldrich & A5306 \\
\hline Glycine & Sigma-Aldrich & G7126 \\
\hline Silver Nitrate & Sigma-Aldrich & S7276 \\
\hline Bialaphos & Gold & B0178 \\
\hline Bhidiazuron & Biotechnology & \\
\hline Acetosyringone & Sigma-Aldrich & D134406 \\
\hline 6-Benzylaminopurine & Sigma-Aldrich & D134406 \\
\hline Zeatin & Phyto & Z860 \\
\hline Indole-3-Acetic Acid & Technologies & \\
\hline & Phyto & I364 \\
\hline Abscisic Acid & Sigma-Aldrich & A1049 \\
\hline
\end{tabular}




\begin{tabular}{|l|l|l|}
\hline Cefotaxime & $\begin{array}{l}\text { Gold } \\
\text { Biotechnology }\end{array}$ & C-104-1 \\
\hline Carbenicillin & $\begin{array}{l}\text { Gold } \\
\text { Biotechnology }\end{array}$ & C-103-5 \\
\hline
\end{tabular}

Table 8 Chemicals Used in Transformation Protocol.

2,4-D: Dissolve $100 \mathrm{mg}$ of 2,4-D in $2 \mathrm{~mL} 1 \mathrm{~N} \mathrm{NaOH}$ and adjust to $100 \mathrm{~mL}$ with $\mathrm{ddH} 20$. Store at $4^{\circ} \mathrm{C}$. No sterilization necessary.

Acetosyringone (AS): Dissolve $0.196 \mathrm{~g}$ of AS in $5 \mathrm{~mL}$ methanol. Add $5 \mathrm{~mL}$ ddH20 to make final volume of $10 \mathrm{~mL}$. Filter sterilize and stoer at $-20^{\circ} \mathrm{C}$ in $1 \mathrm{~mL}$ aliquots.

Bialaphos (3mg/ml): Dissolve $120 \mathrm{mg}$ of bialaphos in $40 \mathrm{~mL} \mathrm{ddH20.} \mathrm{Filter}$ sterilize and store at $4^{\circ} \mathrm{C}$. (Prepare after 2 months) (30 mg in $10 \mathrm{~mL}$ ) Copper Sulfate (10 mM): Dissolve $24.97 \mathrm{mg}$ of copper sulfate pentahydrate in 10 $\mathrm{mL}$ of $\mathrm{ddH} 20$. Store at $4^{\circ} \mathrm{C}$.

Glycine (2 mg/L): $100 \mathrm{mg}$ glycine in $50 \mathrm{~mL}$ ddH20. Filter sterilize and store at $4^{\circ} \mathrm{C}$.

Silver nitrate $(8.5 \mathrm{mg} / \mathrm{ml})$ : Dissolve $340 \mathrm{mg}$ of silver nitrate in $40 \mathrm{~mL}$ ddH20. Filter sterilize and store at $4^{\circ} \mathrm{C}$.

Abscisic Acid $(0.1 \mathrm{mg} / \mathrm{ml})$ : Dissolve $1 \mathrm{mg}$ of $A B A$ in $10 \mathrm{~mL}$ of distilled water. Filter sterilize with .22 um filter and aliquot $500 \mathrm{uL}$ into $1.7 \mathrm{~mL}$ tubes. Store at $20 \mathrm{C}$. 
Vitamin Mix A (1000x): Dissolve 5 g Myo-inositol, 25 mg Nicotinic Acid, 50 mg

Thiamine $\mathrm{HCL}$, and $25 \mathrm{mg}$ Pyridoxine in $50 \mathrm{~mL}$ distilled water. Filter

sterilize with .22 um filter and store at $4^{\circ} \mathrm{C}$.

Vitamin Mix B (1000x): Dissolve 12.5 mg Myo-Inositol and 75 mg Thiamine HCL

in $50 \mathrm{~mL}$ distilled water. Filter sterilize with $.22 \mathrm{um}$ filter and store at $4^{\circ} \mathrm{C}$.

Vitamin Mix C (1000x): Dissolve 5 g Myo-Inositol, 25 mg Nicotinic Acid, 5 mg

Thiamine $\mathrm{HCL}$ and $25 \mathrm{mg}$ Pyridoxine $\mathrm{HCL}$ in $50 \mathrm{~mL}$ distilled water. Filter

sterilize with .22 um filter and store at $4^{\circ} \mathrm{C}$.

\begin{tabular}{|c|c|c|c|c|c|c|c|}
\hline \multicolumn{8}{|c|}{ Consensus Protocol - All Solutions for $1 \mathrm{~L}$} \\
\hline & NG-I & NG-CC & NG-R & NG-S1 & NG-S2 & NG-SH & NG-RG \\
\hline MS Salts & $4.3 \mathrm{~g}$ & $4.3 \mathrm{~g}$ & $4.3 \mathrm{~g}$ & $4.3 \mathrm{~g}$ & $4.3 \mathrm{~g}$ & $4.3 \mathrm{~g}$ & $4.3 \mathrm{~g}$ \\
\hline Sucrose & $68.5 \mathrm{~g}$ & $25 \mathrm{~g}$ & $30 \mathrm{~g}$ & $30 \mathrm{~g}$ & $30 \mathrm{~g}$ & $60 \mathrm{~g}$ & $35 \mathrm{~g}$ \\
\hline Glucose & $36 \mathrm{~g}$ & $10 \mathrm{~g}$ & 0 & 0 & 0 & 0 & 0 \\
\hline L-Proline & 0 & $.7 \mathrm{~g}$ & $.7 \mathrm{~g}$ & $.7 \mathrm{~g}$ & $.7 \mathrm{~g}$ & 0 & 0 \\
\hline MES Salts & 0 & $.5 \mathrm{~g}$ & 0 & 0 & 0 & 0 & 0 \\
\hline Casamino Acid & $1 \mathrm{~g}$ & 0 & 0 & 0 & 0 & 0 & 0 \\
\hline Caseine hydrolysate & 0 & 0 & $1 \mathrm{~g}$ & $1 \mathrm{~g}$ & $1 \mathrm{~g}$ & 0 & 0 \\
\hline 2,4-D $(1 \mathrm{mg} / \mathrm{ml})$ & $1.5 \mathrm{~mL}$ & $1.5 \mathrm{~mL}$ & $1 \mathrm{~mL}$ & $1.5 \mathrm{~mL}$ & $1.5 \mathrm{~mL}$ & 0 & 0 \\
\hline Copper Sulfate $(10 \mathrm{mM})$ & & $10 \mathrm{uL}$ & $490 \mathrm{uL}$ & $490 \mathrm{uL}$ & $490 \mathrm{uL}$ & $400 \mathrm{uL}$ & 0 \\
\hline pH & 5.2 & 5.8 & 5.8 & 5.8 & 5.8 & 5.6 & 5.6 \\
\hline Gelrite & 0 & 0 & $3 \mathrm{~g}$ & $3 \mathrm{~g}$ & $3 \mathrm{~g}$ & $3 \mathrm{~g}$ & $3 \mathrm{~g}$ \\
\hline Agar & 0 & $8 \mathrm{~g}$ & 0 & 0 & 0 & 0 & 0 \\
\hline Sterilization & Filter & Autoclave & Autoclave & Autoclave & Autoclave & Autoclave & \begin{tabular}{|l|} 
Autoclave \\
\end{tabular} \\
\hline Vitamins A (1000x) & $1 \mathrm{~mL}$ & $1 \mathrm{~mL}$ & 0 & 0 & 0 & 0 & 0 \\
\hline Vitamins B (1000x) & 0 & 0 & $1 \mathrm{~mL}$ & $1 \mathrm{~mL}$ & $1 \mathrm{~mL}$ & 0 & 0 \\
\hline Vitamins C (1000x) & 0 & 0 & 0 & 0 & 0 & $1 \mathrm{~mL}$ & $1 \mathrm{~mL}$ \\
\hline Glycine $(2 \mathrm{mg} / \mathrm{mL})$ & 0 & 0 & 0 & 0 & 0 & $2 \mathrm{~mL}$ & $2 \mathrm{~mL}$ \\
\hline Silver Nitrate $(8.5 \mathrm{mg} / \mathrm{mL})$ & 0 & $.2 \mathrm{~mL}$ & 0 & $.2 \mathrm{~mL}$ & $.2 \mathrm{~mL}$ & 0 & 0 \\
\hline Bialaphos $(3 \mathrm{mg} / \mathrm{mL})$ & 0 & 0 & 0 & $.5 \mathrm{~mL}$ & $1 \mathrm{~mL}$ & $.5 \mathrm{~mL}$ & 0 \\
\hline Acetosyringone $(100 \mathrm{mM})$ & $1 \mathrm{~mL}$ & $1 \mathrm{~mL}$ & 0 & 0 & 0 & 0 & 0 \\
\hline BAP & 0 & $10 \mathrm{uL}$ & $200 \mathrm{uL}$ & $500 \mathrm{uL}$ & $500 \mathrm{uL}$ & 0 & 0 \\
\hline Zeatin $(1 \mathrm{mg} / \mathrm{mL})$ & 0 & 0 & 0 & 0 & 0 & $500 \mathrm{uL}$ & 0 \\
\hline IAA $(1 \mathrm{mg} / \mathrm{mL})$ & 0 & 0 & 0 & 0 & 0 & $1 \mathrm{~mL}$ & 0 \\
\hline Thidiazuron $(1 \mathrm{mg} / \mathrm{mL})$ & 0 & 0 & 0 & 0 & 0 & $10 \mathrm{uL}$ & 0 \\
\hline ABA $(.1 \mathrm{mg} / \mathrm{mL})$ & 0 & 0 & 0 & 0 & 0 & $264 \mathrm{uL}$ & 0 \\
\hline Carb & 0 & 0 & 0 & 0 & $100 \mathrm{mg}$ (filter) & $100 \mathrm{mg}$ (filter) & 0 \\
\hline Cefotaxime & & & $250 \mathrm{mg}$ (filter) & $250 \mathrm{mg}$ (filter) & & & \\
\hline Time & $5 \mathrm{Min}$ & 3 Days & 3 Days & 2 Weeks & Until Rapid Growth & Until Shoots & \begin{tabular}{|l} 
Until Roots \\
\end{tabular} \\
\hline & & & & & Fresh Media Every 2 Weeks & Fresh Media Every 3 Weeks & \\
\hline
\end{tabular}

Table 9 Final Consensus Protocol

Media composition and incubation times for consensus protocol. All components added poststerilization should be sterilized through a $0.22 \mu \mathrm{M}$ filter and only once the solution has cooled to $50^{\circ} \mathrm{C}$. 


\section{Consensus Transformation Protocol}

Ears from Hi-II A x B (or reciprocal) lines were harvested when the embryo reached $\sim 1.8 \mathrm{~mm}$ in length, about 9-12 days after pollination. About 3 hours before embryo harvesting was to be completed, $5 \mathrm{~mL}$ of NG-I media was infected from a two day old streak of Agrobacterium until the media measured 0.3 to 0.4 (OD 550) on a spectrophotometer. The media was placed on a horizontal shaker at room temperature and shaken for 3 hours at $120 \mathrm{rpm}$. In a sterile flow hood, ears were submerged in 50\% bleach with 2-3 drops of Tween-20 for 30 minutes, then washed 3 times in autoclaved DI water. A fresh sterile scalpel was used to remove the top $1 / 3$ of the kernels from an ear and the embryos excised with a scoopula. Embryos were placed into a $1.7 \mathrm{~mL}$ tube containing NG-I media without Agrobacterium until embryo collection was complete. Embryos were split amongst $1.7 \mathrm{~mL}$ tubes so that no more than 100 embryos were in a tube. Once embryos from all ears were harvested, embryos were washed three times with fresh NG-I media. Once washing was completed, and the infection media with Agrobacterium had shaken for at least 3 hours, $1 \mathrm{~mL}$ of Agrobacterium solution was added to each tube of embryos and the solution inverted to mix. Embryos were infected for 5 minutes, with the tubes inverted every minute to mix. After infection, the tubes were poured onto individual plates of NG-CC media and excess infection media was removed with a pipette. Embryos were then evenly distributed around the plates and flipped scutellum side down. Plates were then sealed and placed in a $20^{\circ} \mathrm{C}$ incubator for 3 days. 
Following co-cultivation, embryos were transferred to NG-R media for another 3 days at $28^{\circ} \mathrm{C}$. Embryos were then transferred to NG-S1 to begin selection. Embryos were broken apart using forceps as they were transferred to the new plates, and callus allowed to develop for 2 weeks. Following the first two weeks of selection, callus was transferred to NG-S2. Only callus that was obviously growing was transferred to new plates. Callus was allowed to grow on NG-S2 media until rapid callus development was observed, about 4-8 weeks, with transfer to fresh media occurring every 2 weeks.

Following selection, friable well-growing callus could be seen that was at least $1 \mathrm{~cm}$ in diameter. All callus pieces were then transferred to NG-SH media to develop shoots. Callus was maintained on NG-SH media until shoots could be observed emerging from the callus, 2-6 weeks. Shoots were then transferred to NG-RG media in culture tubes and placed in a growth chamber under a 12-hour light/dark cycle until plantlets developed full roots, 2-3 weeks. Plantlets were then moved to soil in a growth chamber until the third leaf stage, before being moved to the greenhouse.

\section{Results}

\section{Tests of Consensus Protocol}

Before the consensus protocol was used for large-scale transformation, it was first tested in small experiments. One of the reasons for the cautious approach was that most patents explicitly stated they were designed around proprietary maize lines. As academic transformation is performed on line Hi-II, 
which will be slower growing and less prolific, it was anticipated that timings would need to be adjusted. Additionally, as most patents were not explicit in exact callus manipulation and appearance, it was expected that the inclusion of growth regulators in transformation would lead to differences in appearance than standard techniques. As the trials would be with a low number of embryos, it was not expected that a transformation event would occur; however, the process could be optimized with any false-positive callus that was carried through.

The first transformation trial differed from the final consensus protocol in multiple ways. First, the antibiotic treatments consisted of $100 \mathrm{mg} / \mathrm{L}$ carbenicillin throughout the protocol. Second, the resting media step was carried out for 10 days, as this was the standard time being used in the lab for the standard protocol. Lastly, selection was carried out for 4 weeks total, with 2 weeks spent on S1 and S2 each.

The first problem observed involved Agrobacterium growth after cocultivation. Bacterial growth could be observed throughout the process, up until plantlets were transferred to soil. It was often discovered that despite callus appearing seemingly free of bacterial growth, once callus began to die in selection, Agrobacterium growth would reappear. As a result, it was noted in the next experiment to switch to cefotaxime in the resting step.

The experiment also revealed that the resting step should be drastically reduced. As can be observed in figure 1, the inclusion of BAP in the resting media induced shoot growth, as well as callus formation. While this suggested that the hormones were successful in triggering shoot formation, embryos were 
also more similar to type I callus than type II and difficult to divide on plates. When finally moved to selection plates, shoots were removed with sterilized scissors, and callus broken apart with forceps.

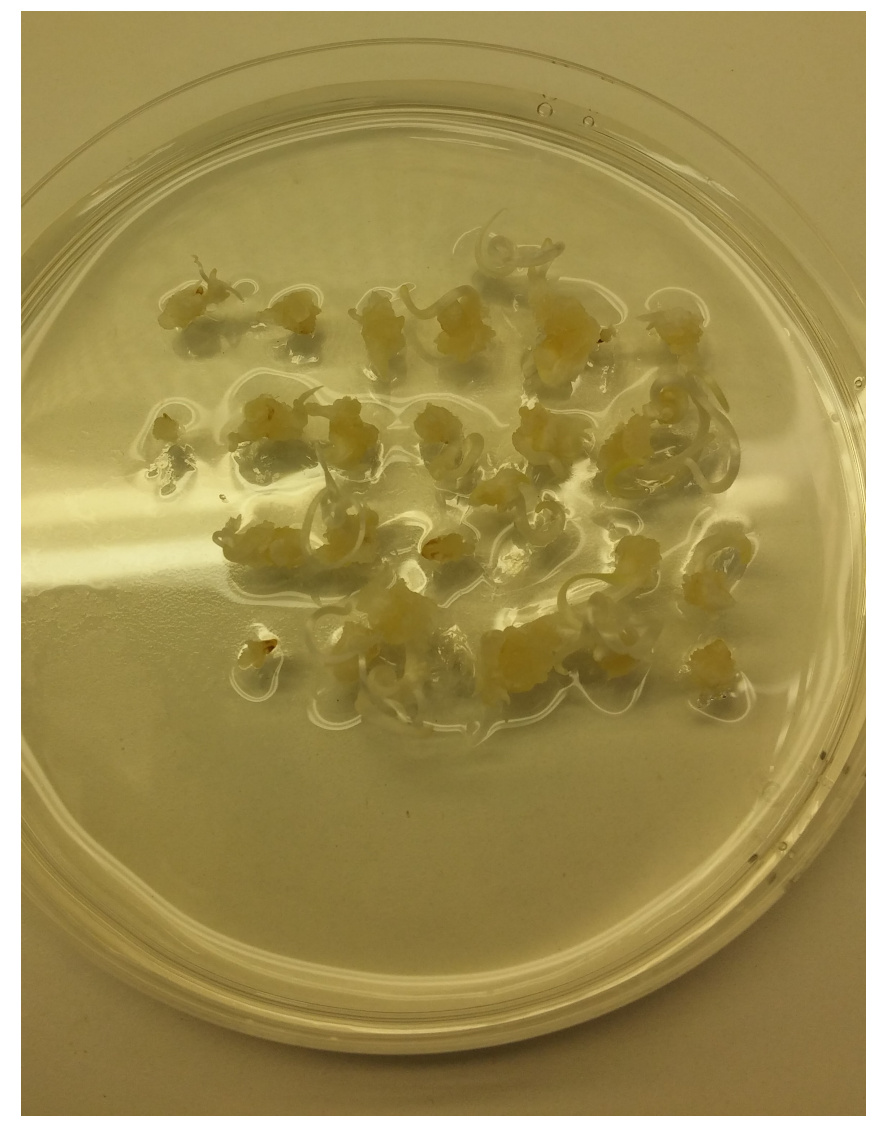

Figure 1 Callus Growth After 10 Days on Resting Media

In addition to the improved shoot growth with the media, the new media formulations also resulted in more media uptake during growth. An example of this can be seen in figure 1 , where callus can be seen to almost 'sink' into the media. This is contrary to the traditional methods, where visual media uptake is not usually observed until shooting. This is of note as traditional shooting media is also when media formulations are switched to MS media base, suggesting that 
MS media allows for increased callus growth. The consequence of the improved growth is there is also additional uptake of selection agent during the selection stage. Indeed, callus was often observed rapidly turning dark brown or black during selection, as can be seen in figure 2 . This is interesting because in traditional methods callus will often slowly turn brown during selection, sometimes taking weeks to fully die.

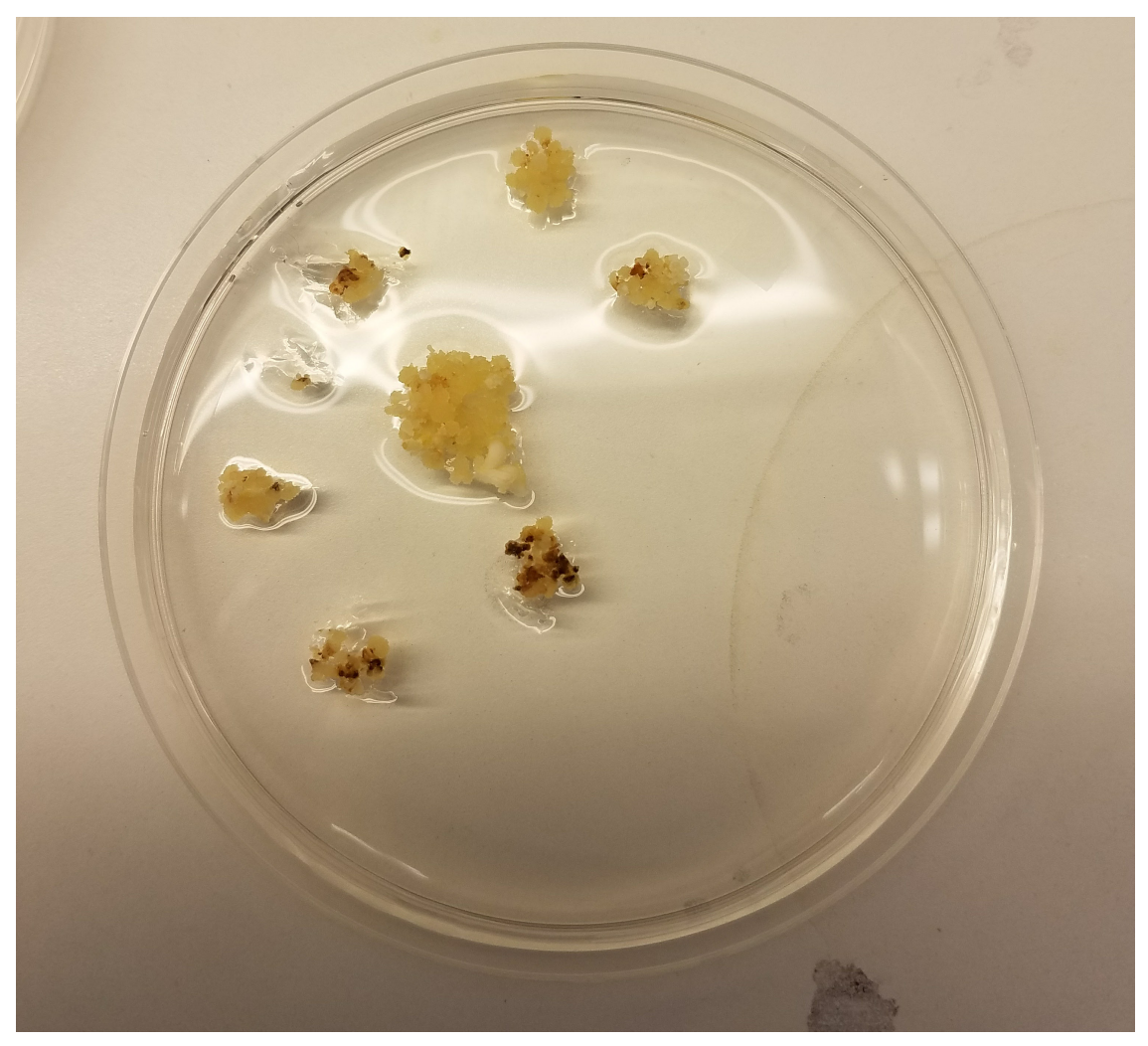

Figure 2 Callus Selection After 2 weeks of S2 Selection

The original test of the consensus protocol did not result in any transformed plants; however, three false-positive plants were allowed to go through the full regeneration process. Regeneration, from embryo dissection until 
plantlets were planted in soil, took approximately 3 months. Additionally, regenerated plants were healthy with no apparent abnormalities.

The second transformation trial included cefotaxime in the regeneration step, and a 5 day resting period. While the Agrobacterium growth was reduced in this trial, it was still occasionally spotted in decaying callus tissue. The rest period was still too long. Again, callus was most similar to type I, and difficult to break apart. Additionally, shoots were again found before selection had occurred. Once the shoots are removed and callus pieces broken apart, callus does transition to more type II like. However, this may reduce transgenic tissue growth. As the callus is not originally friable, when a sector of callus is killed, a large region will die potentially removing transgenic sectors.

To experiment with whether BAP should only be added once in the selection step, a third transformation trial was carried out with only a 3 day rest period. Additionally, in this trial a small $(\sim 70)$ number of B73 embryos were included to determine if the protocol was capable of also regenerating previously unregenerable lines. While the shortened rest period did improve the callus formation, the presence of type I like callus was still undesirable. Additionally, as before, there was often emergence of Agrobacterium in the selection stage. The protocol was altered to include cefotaxime in both the resting and first selection stage. The B73 embryos, while noticeably different from the Hi-II embryos, were capable of regeneration and a healthy plantlet was recovered from the process in about 2.5 months. 
There are two options to improve the amount of type II callus production. First, the BAP hormone can be delayed until the selection stage. This option should allow traditional callus formation, which would allow better selection. As this media should develop more traditionally, a longer resting period can be included to fully induce type II callus before exposure to growth regulators. The other option is to continue with the BAP containing media, but to divide the type I appearing callus earlier in the process and more often. Additionally, it may then be advisable to maintain the selection process longer, with callus only being moved to shooting once readily apparent type II callus has formed. This option may be the strategy that Pioneer has adapted. As can be seen in Table 6, most of the Pioneer protocols call for switching to shooting media once sufficient growth has been observed. Often in the patents this is defined as well-growing callus at least $1 \mathrm{~cm}$ in diameter. As can be seen in Table 6, both options appear to have been considered in industry patents with the inclusion of BAP occurring in the recent and most-filed patents.

To examine whether better callus could be obtained before selection without BAP, a fourth transformation trial was conducted. In this experiment, cefotaxime was included in both resting and the first selection steps. Additionally, BAP was not included in the media until selection, and callus was maintained on resting media until more traditional looking callus was observed. In these trials, no Agrobacterium overgrowth was observed, and there was no noticeable reduction in callus health with the addition of cefotaxime. Additionally, the later addition of BAP induced a more typical type II callus response from $\mathrm{Hi}-\mathrm{Il}$ embryos 
before entering selection. While the selection stage was delayed, the increase in callus formation may be worth the increase in overall time. When the delayed BAP addition was utilized with B73 embryos, however, no callus was formed, and embryos were not able to survive until selection. This suggests that for recalcitrant lines, the addition of growth hormone may be required for regeneration from the beginning of the process.

In all trials, the efficacy of the shooting media was readily apparent. Compared to traditional media, which does not include growth regulators and will often take 4-6 weeks to begin shoot formation, the consensus shooting media NG-SH caused shoots to begin appearing as early as one week after transfer. For most readily growing callus, shoots were observed around 2-3 weeks after transfer. Additionally, it was found that by switching to new shooting media every 3 weeks, instead of every 2 as in selection, shoot formation was improved. Additionally, moving poorly growing callus from traditional media for other experiments to NG-SH induced shoot formation that was not occurring beforehand. It should be noted, however, that unlike traditional shooting media that continues callus proliferation on a plate, callus on NG-SH media does not continue to spread outward. The result is that fewer plants can usually be obtained from a single event.

\section{Discussion}

The transformation process in maize is complicated, with a large number of variables present at each step. In order to truly investigate the ideal procedure, one would need to investigate each component and method with a large number 
of biological replicates. As the process, even when accelerated, takes many months, this is too expensive and time consuming for the standard academic lab. Indeed, the number of variables involved is probably too large even for industry labs to truly investigate each step exhaustively. Interestingly, a review of patents reveals that the industry sector is attempting these experiments at a small scale. While the patents usually mention small sample sizes in most experiments, many alterations were maintained in subsequent patents years later.

The most consistent change across all of the observed patents was the switch from an N6 based medium for inoculation through selection, to an MS based medium. Interestingly MS salts were used by all companies except Japan Tobacco Company, which has used LS medium since at least 1996. In practice, it is difficult to know what improvement the switch from N6 to MS salts has, as one would need to only switch this variable to be able to observe any effect, and in this study multiple factors were altered in each media. However, as callus growth was certainly not worse than before, and there is such a strong consistency across industry, it is difficult to make a case not to make the switch.

The change that is most noticeable in the protocol is the inclusion of growth regulators. In previous protocols, callus is maintained on different sucrose concentrations to trigger alternate developmental stages. While this technique works, it is extremely time-consuming. The inclusion of growth regulators to accelerate the process has the potential to greatly reduce the time and cost of the process. Indeed, if the patent claims are to be believed, it may be possible to recover transgenic plants in as little as 4-8 weeks (Rout et al., 2013). Of course, 
many industry patents include other improvements such as automation and improved commercial lines that make it difficult to achieve such results in an academic setting. So, while it may be difficult to achieve a regenerated plant in 4 weeks in an academic lab, it is apparent that the inclusion of growth regulators has the potential to greatly improve the process.

In practice, the application of growth regulators to transformation media results in an obvious morphological change in growth. As details are often sparse in patents, it is difficult to determine whether these are normal results when hormones are included early in the transformation process. However, as BAP is included as early as resting media by both Monsanto and Pioneer (table 3), it is apparent that the major producers of transgenic maize are using the hormone strategy for commercial production. The limitation in details in patents also makes it difficult to know if there are changes to the technique to deal with the new callus morphology. As a result, it may be best to attempt a hybrid strategy, where traditional techniques can be applied to the new formulation of media. This strategy, where more traditional friable callus is first grown before the application of hormones, may lead to a slower process, but is more consistent with the techniques already utilized in academic transformation labs.

Another aspect that should be discussed is the use of alternate modes of selection throughout the industry sector. In academia, the selective agent has typically been bialaphos. While the bialaphos selection strategy has been successful for decades, it is slow acting, and can be difficult to distinguish inhibited tissues. In the case of industry protocols, many utilize the selective 
agent glyphosate. While glyphosate has not been used in the current study, it is worth considering the effect that such a change may have. For instance, the use of growth regulators in the selection media resulted in false-positives when used with $\mathrm{Hi}-\mathrm{Il}$ embryos and bialaphos selection. As bialaphos often requires multiple rounds of selection before true positive sectors can be found, it may be that the use of bialaphos is leading to the many false-positive plants recovered. Extending the amount of time that callus is exposed to the selection agent prior to the addition of growth hormones may also increase the chances of positive selection, but also will increase the transformation time as well.

The line of maize being used should also be considered when making comparisons to industry protocols. While Hi-II has traditionally been utilized in academic settings, it is clear from examining industry sector patents that commercial lines are often utilized in private industry. The ability to use commercial hybrid lines would have a large effect on embryo growth. In the experiments with $\mathrm{B} 73$, which has a greater vigor than $\mathrm{Hi}-\mathrm{II}$, but would be less vigorous than a commercial hybrid, embryos developed much more rapidly. Additionally, Hi-Il plants are consistently difficult to maintain, and obtaining high quality embryos can be difficult. The ability to utilize a commercial hybrid would make producing high numbers of embryos much easier. The line $\mathrm{Hi}-\mathrm{Il}$ is chosen, however, due to its ability to form high amounts of type II callus. Other lines, including B73, are generally considered to be recalcitrant to transformation, which is why they are not used. Many protocols suggest that by including a growth hormone, such as BAP, that lines that were previously considered to be 
recalcitrant could now be transformed. The work here, suggests that this may be the case as B73 could be regenerated with the consensus protocol, though only when growth hormone was supplemented at the beginning of the process.

It should also be mentioned at this stage that there is often little mention of transformation efficiency in industry patents. As a result, it is difficult to gauge how much an improvement in transformation time comes at the expense of transformation efficiency. There cannot be too drastic of a reduction, however, as an extremely low transformation efficiency would result in a high cost for embryo production. Transformation efficiency may also be omitted due to a lack of standard for the measurement. Many list the frequency as the ratio of transformed events to extracted embryos; however, this number is rarely consistent. For instance, transformation efficiency may be increased simply by only transforming embryos within a certain size restriction, despite embryos outside of this limit being extracted from an ear. While it is true that embryos outside of this limit are less likely to result in a transformed plant, there is little benefit other than improving the final efficiency number, from excluding already extracted embryos from the process. Additionally, embryos that fail to develop into callus, or callus that is lost due to contamination during the process could be excluded from the final numbers to inflate the efficiency number as well. As there is little standard for this measurement, and a high amount of variability in how it is recorded, the actual transformation efficiency number may be of little value.

Maize transformation has long been a difficult and costly process. The work shown here suggests that by manipulating the growth media it may be 
possible to improve the process. By combining the improved media compositions with glyphosate selection, the process may be even further accelerated.

\section{References}

Akula, A., Duncan, D., Lowe, B., Mann, M., Peterson, W., Rout, J., Songstad, D., Wilks, J., and Zhang, W. (2015). Methods for Producing Transgenic Plants. 8,962,326 B2.

Armstrong, C.L., Green, C.E., and Phillips, R.L. (1991). Development and availability of germplasm with high Type II culture formation response. Maize Genetics Cooperation Newsletter 92-93.

Cai, T., Subbarao, S., Wan, Y., Yingling, R., Mann, M., Otal, A., and Songstad, D. (2004). Methods for Corn Transformation. US 2004/024407.

Cai, T., Subbarao, S., Wan, Y., Yingling, R., Mann, M., Otal, A., and Songstad, D. (2014). Methods for Corn Transformation. 8,759,609 B2.

Cho, M.-J., Ellis, S., Gordon-Kamm, W., and Zhoa, Z.-Y. (2014). Methods and Compositions for Producing and Selecting Transgenic Plants. US 2014/0173775 A1.

Frame, B.R., Shou, H., Chikwamba, R., Zhang, Z., Xiang, C., Fonger, T.M., Pegg, S.E., Li, B., Nettleton, D., and Wang, K. (2002). Agrobacterium tumefaciens-Mediated Transformation of Maize Embryos Using a Standard Binary Vector System. Plant Physiology 129, 13-22.

Gordon-Kamm, W., Klein, T., Lowe, K., McBride, K., Scelonge, C., Wang, B.-B., Wang, N., and Wu, X. (2011). Methods and Compositions for the Introduction and Regulated Expression of Genes in Plants. US 
2011/0167516A1.

Imayama, T., Hiei, Y., and Ishida, Y. (2016). Agrobacterium Bacterium to be Used in Plant Transformation Method. US 2016/0083737 A1.

ISAAA (2016). Global Status of Commercialized Biotech/GM Crops: 2016 (Ithaca, NY).

Ishida, Y. (2007). Method for Improving Plant Transformation Efficiency by Adding Copper lon. US 2007/0163007.

Ishida, Y., Saito, H., Ohta, S., Hiei, Y., Komari, T., and Kumashiro, T. (1996). High efficiency transformation of maize (Zea mays L.) mediated by Agrobacterium tumefaciens. Nature Biotechnology 14, 745-750.

Lee, H., and Zhang, Z.J. (2014). Agrobacterium-Mediated Transformation of Maize (Zea mays) Immature Embryos. In Methods in Molecular Biology, pp. $273-280$.

Lowe, K., Wu, E., Wang, N., Hoerster, G., Hastings, C., Cho, M.-J., Scelonge, C., Lenderts, B., Chamberlin, M., Cushatt, J., et al. (2016). Morphogenic Regulators Baby boom and Wuschel Improve Monocot Transformation. The Plant Cell 28, 1998-2015.

Mehlo, L., and Zhao, Z.-Y. (2012). Method for Regulating AgrobacteriumMediated Transformation. 8,143,484 B2.

Rout, J. (2013). Plant Transformation Without Selection. 8,581,035 B2.

Rout, J., Lowe, B., Purcell, J., Spelletich, A., Spencer, M., and Way, M. (2013). Methods for Rapidly Transforming Monocots. US 2013/0239253 A1. Wu, X., Cho, M.-J., and Zhao, Z.-Y. (2013). Methods for Improving Monocol 
Transformation. 8,404,930.

Zhong, H. (2015). Composition and Method for Enhancing Plant Transformation. US 2015/0113681. 


\title{
Chapter 2 Demonstration of $\Phi C 31$ Integrase and
}

\author{
Excisionase Activity in Zea mays
}

\begin{abstract}
Recombinase systems, which have been utilized in transgenic research for decades, allow researchers to manipulate genetic sequences precisely. Cre recombinase, the most widely used recombinase system in plants, will catalyze the kinetically favorable excision reaction of transgenes between appropriately oriented loxP sites. As a result, transgene insertion into loxP sites is reversible. To remedy this problem, the recombinase system $\Phi C 31$ has been tested in maize (Zea mays) as an alternative to the Cre/lox system. ФC31 recombination is unidirectional and the directionality can be controlled through the presence or absence of the excisionase protein. In this work, ФC31 integrase and excisionase are shown to be functional both transiently and integrated into the genome of maize. By utilizing a GUS reporter construct, integrase is shown to have a high rate of activity in maize cells. This system should facilitate targeted transformation into recipient sites in the maize genome or on minichromosomes.

\section{Introduction}

Plant biotechnology has become an important tool for agricultural research. However, despite the first transgenic plants being produced over two 
decades ago, there are still numerous issues with the technology. Many of these issues, such as varying transgene expression and the introduction of multiple copies of a transgene, can be solved by utilizing a site-directed integration scheme. In this strategy, a recombinase system is utilized that can introduce a transgene to a pre-determined genomic location. The most common recombinase system utilized in plants is the bacteriophage P1 Cre/lox system. Cre recombinase recognizes the $34 \mathrm{bp}$ lox recombination sites and, depending on the orientation of sites, has been shown to result in insertion, deletion, or inversion of sequences in plant cells (Dale and Ow, 1991; Medberry et al., 1995; Vergunst et al., 1998). A disadvantage of the Cre/lox system, however, is the sequence of lox sites and hence the ability to recombine is maintained after each reaction. As a result, site-directed insertion of transgenes through recombination at lox sites will result in a transgene flanked by lox sites. These sites are then able to recombine and excise the newly introduced transgene, and are indeed kinetically favored to do so, because of the proximity of cis sites compared to trans interactions needed for insertion. To overcome this problem, mutant lox recombination sites have been used for site-specific integration in plants (Albert et al., 1995). By utilizing mutant lox sites, the result of recombination is a single transgene flanked by a non-functional lox site and a wild-type version on the excised DNA. While these are useful for a single transgene insertion, inclusion of an additional transgene at the same genetic locus would require insertion into the inefficient wild-type lox site. 
The ФC31 recombination system from Streptomyces phage ФC31 (Thorpe and Smith, 1998) would alleviate the issues currently found with the Cre/lox system. The recombination system consists of two proteins, integrase and excisionase. $\Phi C 31$ integrase recognizes attB and attP sites and catalyzes recombination between them (Smith et al., 2004). However, because the attB and attP sites are not identical in sequence, the resulting sites after recombination, att $L$ and att $R$, are not recognized by $\Phi C 31$ integrase for recombination, thus resulting in a unidirectional reaction. In order for successful recombination of attL and attR sites to occur, an additional protein, the recombination directionality factor (ФC31 excisionase) (Khaleel et al., 2011), must be included that binds to $\Phi С 31$ and causes the reverse recombination reaction to occur. Additionally, as the minimal sizes of the attB and attP sites are only 34 bp and 39 bp respectively (Groth et al., 2000), they are small enough for easy manipulation during transgene design. Additionally, alternative attP and attB sites can be produced by altering the 2 bp overlap sequences of the sites, allowing for multiple attP and attB sites to be utilized in one target location (Colloms et al., 2013). The ability to target multiple attB or attP sites in one reaction would allow for several transgenes to be introduced in a specific orientation.

$\Phi C 31$ integrase has been shown to be functional in a variety of plant systems (Gils et al., 2008; Lutz et al., 2004; Rubtsova et al., 2008). ФC31 excisionase has not been tested in plant tissues; however, it was shown to be 
functional in mammalian cells (Farruggio et al., 2012) demonstrating the reversal of ФC31 integration in eukaryotic cells. By combining both $\Phi C 31$ integrase and excisionase, a full system can be developed for transgene manipulation.

In this study, $Ф C 31$ integrase and excisionase are introduced into maize both through full transformation and with transient expression. To determine activity, reporter plasmids are introduced through particle bombardment, which will only express GUS when in the presence of a functional recombinase. Both integrase and excisionase are shown to be functional in maize embryos in transgenic lines and transiently.

\section{Materials and Methods}

\section{Construction of integrase and excisionase plasmids}

To produce an integrase plasmid for maize transformation, plasmid pICH13130 was obtained from the Gils lab (Rubtsova et al., 2008) which contains the Streptomyces $\Phi \mathrm{C} 31$ integrase protein driven by the maize ubiquitin I promoter (Christensen et al., 1992). A 300 bp intron from Petunia hybrida has been inserted into the integrase sequence, and a nuclear localization signal fused to the 3' end. The integrase sequence, along with promoter and terminator, was cloned into the binary transformation vector pTF101.1 to form plasmid pTFPhiC31 (Figure 3). 


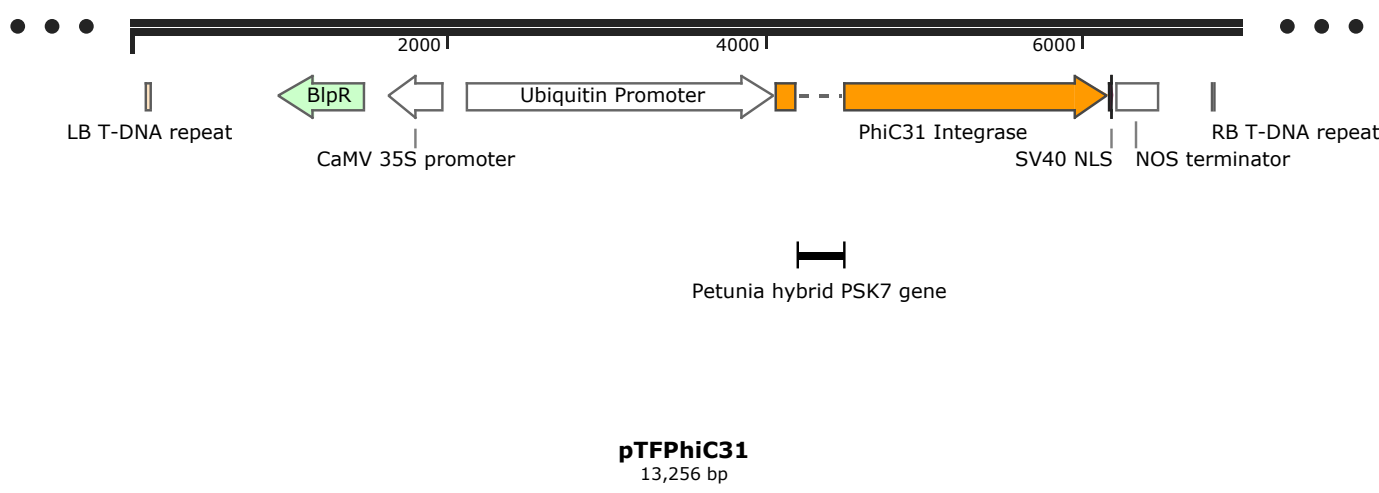

Figure 3 pTFPhiC31

Transfer DNA of plasmid pTFPhiC31. pTFPhiC31 consists of a bialophos selection gene for selection driven by the 35S promoter, and the $\Phi \mathrm{C} 31$ integrase sequence driven by the ubiquitin promoter.

Excisionase was obtained from the Calos lab in plasmid pCS-kRI (Farruggio et al., 2012). As both integrase and excisionase must be present for excision, the plasmid contains $Ф C 31$ excisionase fused to $\Phi C 31$ integrase via a P2A peptide. The integrase/excisionase sequence was cloned into a plasmid containing the maize ubiquitin I promoter and NOS terminator to form plasmid p56IntExc. The promoter, integrase/excisionase sequence, and terminator was then cloned into the binary transformation vector pZY101 to produce pZY56IntExc (Figure 4). 

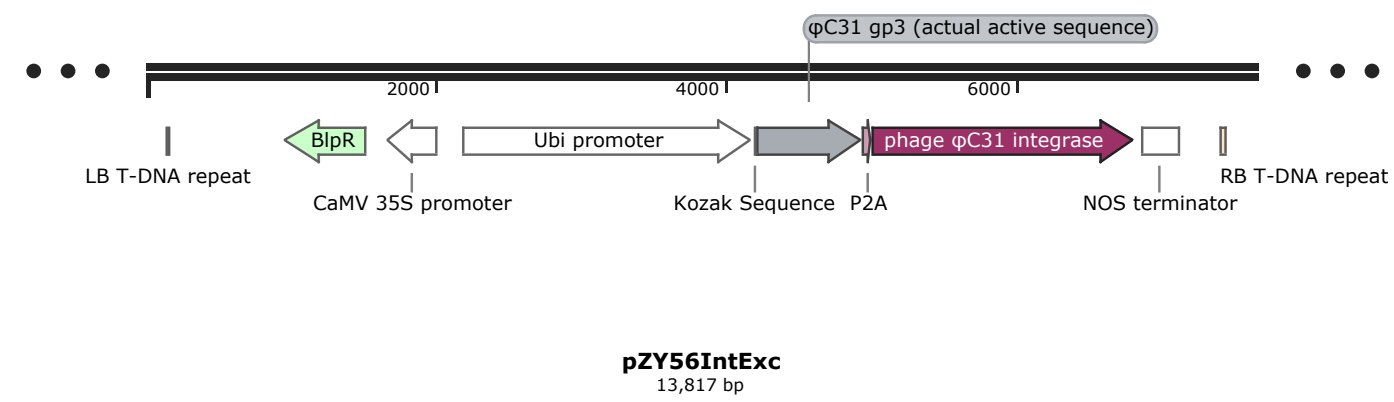

Figure 4 p56IntExc

Transfer DNA of plasmid pZY56IntExc. Plasmid pZY56IntExc contains a bialaphos selection gene driven by the $35 \mathrm{~S}$ promoter, as well as the $\Phi \mathrm{C} 31$ integrase and excisionase sequences driven by a ubiquitin promoter, and linked by a P2A linker sequence.

\section{Maize Transformation of Integrase and Excisionase}

Agrobacterium tumefaciens strain EHA101 was used for maize transformation. Maize Hi-ll embryos were extracted 10-12 days after pollination when embryos were 1.5-2 $\mathrm{mm}$ in length. Selection and regeneration were carried out as described in Vega et al., (2008b). After regeneration, plants were screened via PCR and positive events sequenced via Sanger sequencing of PCR products.

\section{Construction of Integrase and Excisionase Reporter Constructs}

To test the function of $\Phi C 31$ integrase and excisionase, reporter constructs were designed that would only express in the presence of an active recombinase. Reporter constructs were designed to contain a GUS reporter in the reverse orientation behind the maize ubiquitin I promoter. The GUS sequence was flanked by attachment sites that were in opposite orientation so 
that, in the presence of the recombinase, the intervening sequence would be inverted (figure 5).

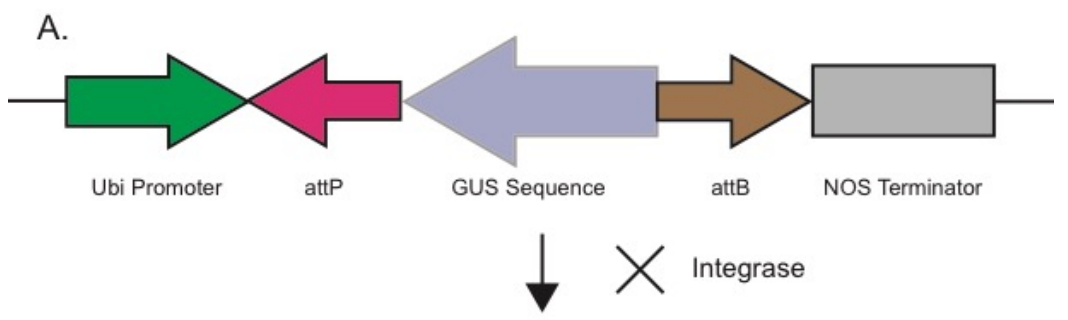

B.
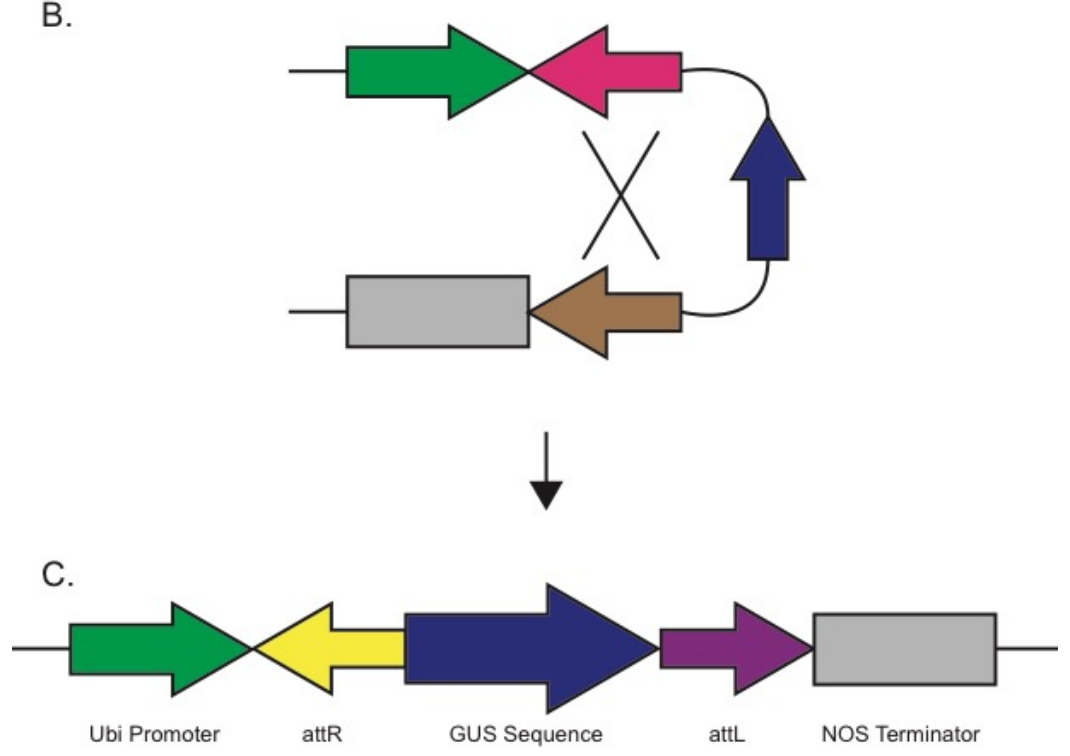

Figure 5 Reporter Strategy for Demonstrating Recombinase Activity

Strategy for examining recombinase activity in maize. (A) A plasmid is synthesized consisting of a ubiquitin promoter, attachment sites, GUS sequence, and NOS terminator. The attachment sites are oriented in opposite orientation from each other, and the GUS reporter is in the antisense direction. (B) Upon introduction of the recombinase, the attachment sites will be oriented in the same orientation and recombination will occur. (C) After recombination, the GUS sequence will be in the sense orientation and thus capable of producing GUS protein. While integrase activity is shown, excisionase activity can be examined with the same strategy. 
To produce a test plasmid for integrase activity, primers were designed containing attachment sites that amplified GUS from plasmid pENTR Gus (Invitrogen). The PCR product was cloned into plasmid pWY56C to produce p56AntiGus (Figure 6).

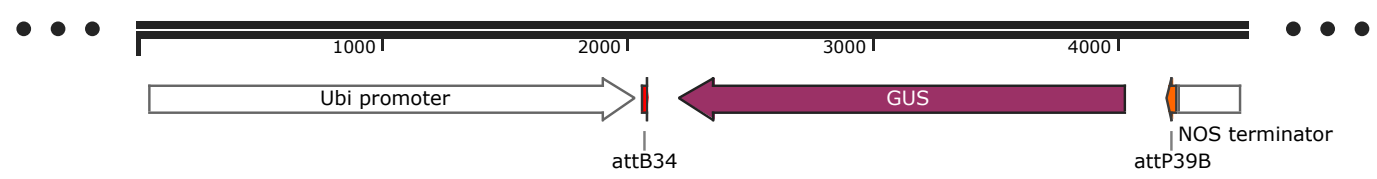

p56antigus
$6938 \mathrm{bp}$

Figure 6 p56AntiGus

Test plasmid for integrase activity. A GUS reporter in the reverse orientation is flanked by opposing attP and attB sites and driven by the maize ubiquitin I promoter.

To test excisionase activity, a plasmid was designed around the same strategy as p56AntiGus but included attL and attR sites in place of attP and attB sites. The attachment site GUS cassette was synthesized (GeneWiz) and subcloned into plasmid pWY56C to produce plasmid p56LAntiGusR (Figure 7). 


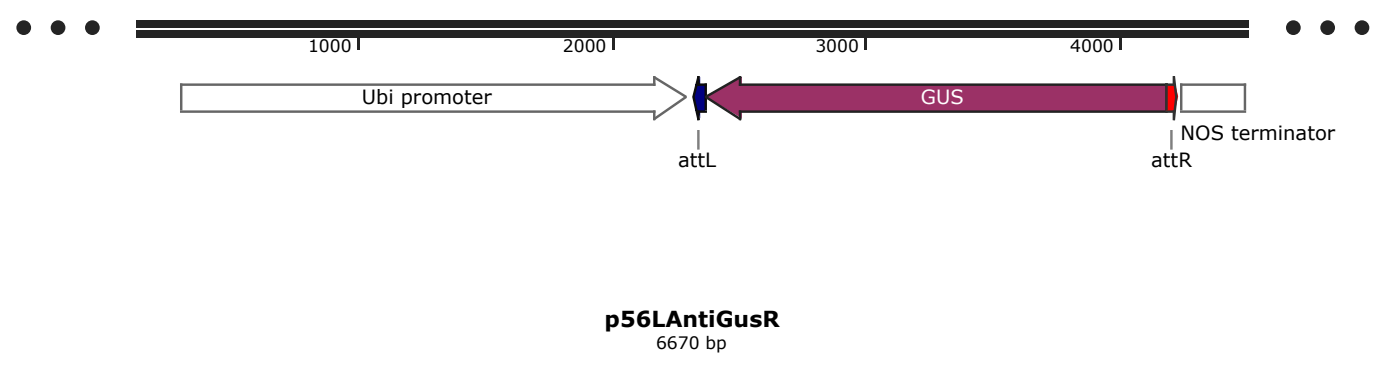

Figure 7 p56LAntiGusR

Test plasmid for excisionase activity. A GUS reporter in the reverse orientation is flanked by opposing attL and attR sites and driven by the maize ubiquitin I promoter.

\section{Particle Bombardment}

Particle bombardment was performed on embryos extracted 10-12 days after pollination measuring 1.5-2 $\mathrm{mm}$ which were allowed to rest on $\mathrm{N} 6$ media for 3-5 days (Songstad et al., 1996). About 4 hours prior to bombardment, embryos were placed on osmotic medium to prepare for bombardment. About $1 \mu \mathrm{g}$ of DNA of each construct was loaded onto $0.6 \mu \mathrm{M}$ gold particles (Bio-Rad) irrespective of whether cobombardment was performed. Bombardment was performed in a Biolistic PDS-1000/He Particle Delivery System (Bio-Rad) using 650 psi rupture disks. Embryos were placed onto fresh N6 media about 1 hour following bombardment.

\section{GUS Staining}

GUS staining was performed 3-5 days after bombardment. Embryos were stained using the Beta-Glucuronidase Reporter Gene Staining Kit (SigmaAldrich) without a fixation step. Embryos were stained up to 3 days before imaging with a Leica M205F A stereoscope. 


\section{Results}

\section{Transient Expression of Integrase and Excisionase}

To examine whether $\Phi C 31$ integrase and excisionase were functional in maize tissues, they were first tested transiently with particle bombardment. Recombinase expression plasmids, as well as reporter plasmids, were cobombarded into embryos to test for activity. As a positive control for bombardment, plasmid pACT1F, which contains GUS under the constitutive actin promoter, was introduced to a small subset of embryos. Additionally, to ensure that recombination sites were indeed specific, reporter constructs were also cobombarded with the opposite recombinase. 


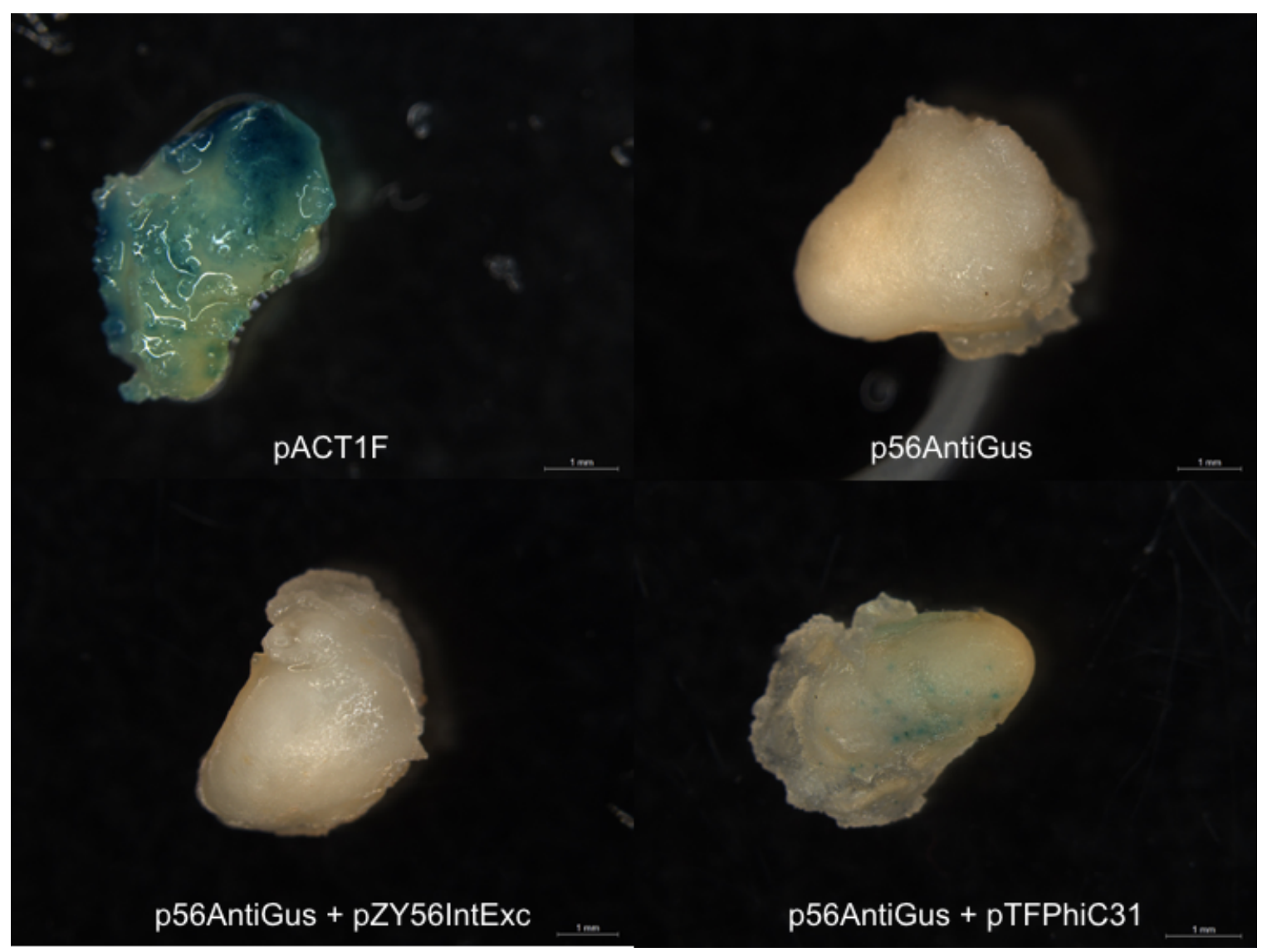

Figure 8 Transient Integrase Activity in Maize

Transient activity of $\Phi C 31$ integrase activity was investigated through bombardment experiments.

(Top Left) Success of bombardment was verified by bombarding an actin-GUS reporter construct. (Top Right) Introducing GUS in the antisense direction does not lead to GUS signal. (Bottom Left) No GUS signal is observed when excisionase is cobombarded with an inverted GUS construct containing attP and attB sites. (Bottom Right) GUS activity is observed when $\Phi C 31$ integrase is cobombarded with an inverted GUS construct. 


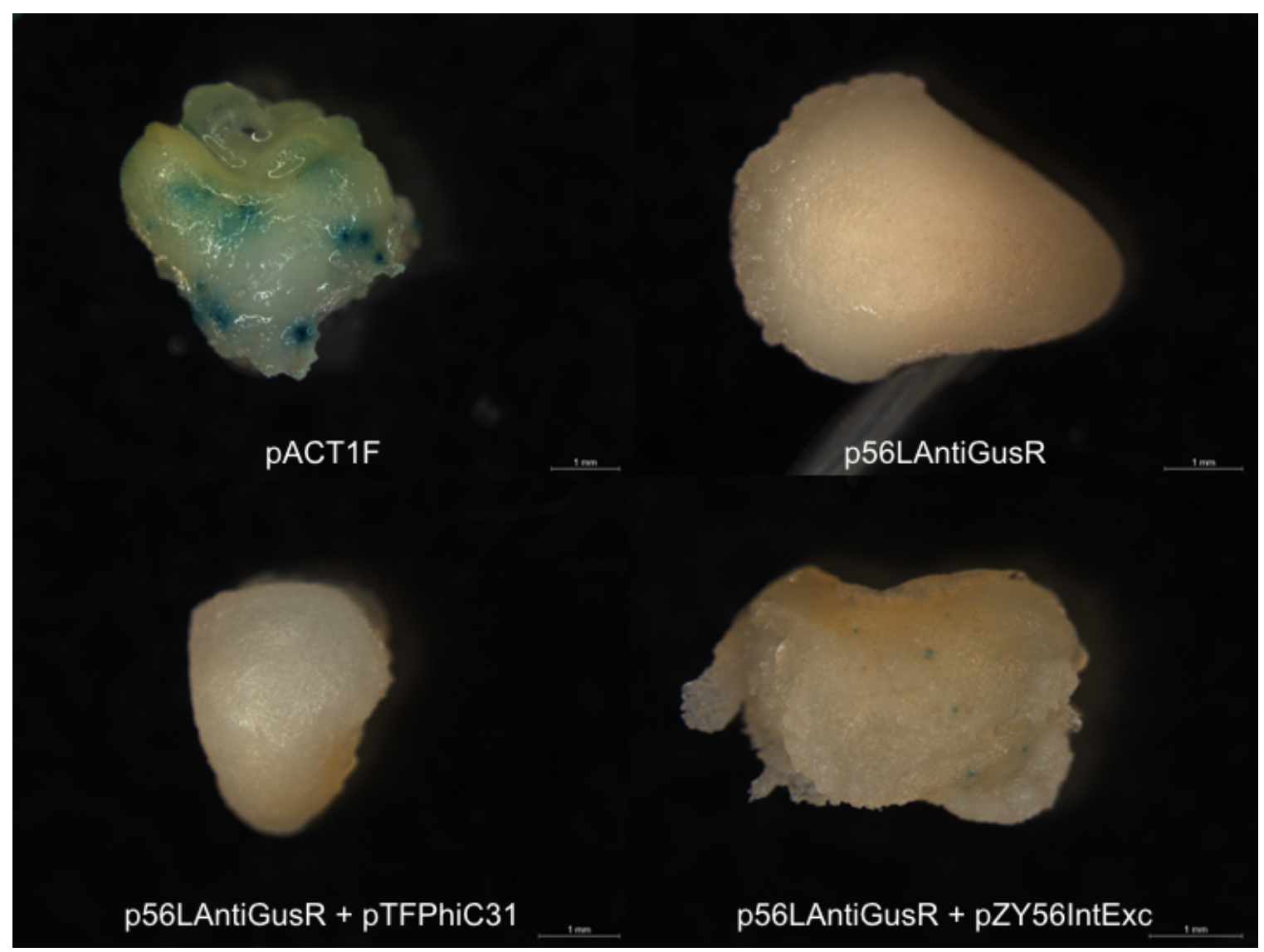

Figure 9 Transient Activity of Excisionase in Maize

Bombardment experiments to investigate $\Phi C 31$ excisionase activity when introduced transiently into maize embryos. (Top Left) Success of bombardment is demonstrated by introducing an actinGUS reporter construct to a subset of embryos. (Top Right) Lack of GUS activity when an antisense GUS reporter is introduced which contains attL and attR sites. (Bottom Left) Lack of GUS activity when excisionase-specific attachment sites are introduced alongside of integrase. (Bottom Right) Demonstration of activity of $\Phi C 31$ excisionase when introduced transiently. GUS expression can be seen resulting from inversion of the GUS sequence.

\section{Activity of $\Phi C 31$ Integrase and Excisionase when Transformed into Maize}

Integrase and excisionase were also transformed into maize line $\mathrm{Hi}-\mathrm{Il}$ to examine activity. Transformation of each construct proved difficult, with recovery 
of events from pTFPhiC31 particularly so. In multiple cases resistant plants were recovered following regeneration that did not contain an intact transgene. This difficulty could be a result of the large size of the transformation construct, or may be evidence of instability within bacterial cells.

One event of both integrase and excisionase were recovered and found to be intact, INT7 and EXC1, respectively. These lines did not appear to contain any abnormalities, and behaved normally in greenhouse conditions. The flowering of INT7 did not nick correctly, and could not be selfed; however, this was normal in the greenhouse for $\mathrm{Hi}-\mathrm{Il}$ at the time. For this reason, embryos resulting from a cross from transgenic T0 plants onto standard $\mathrm{Hi}-\mathrm{Il}$ were used to examine transgene activity. 


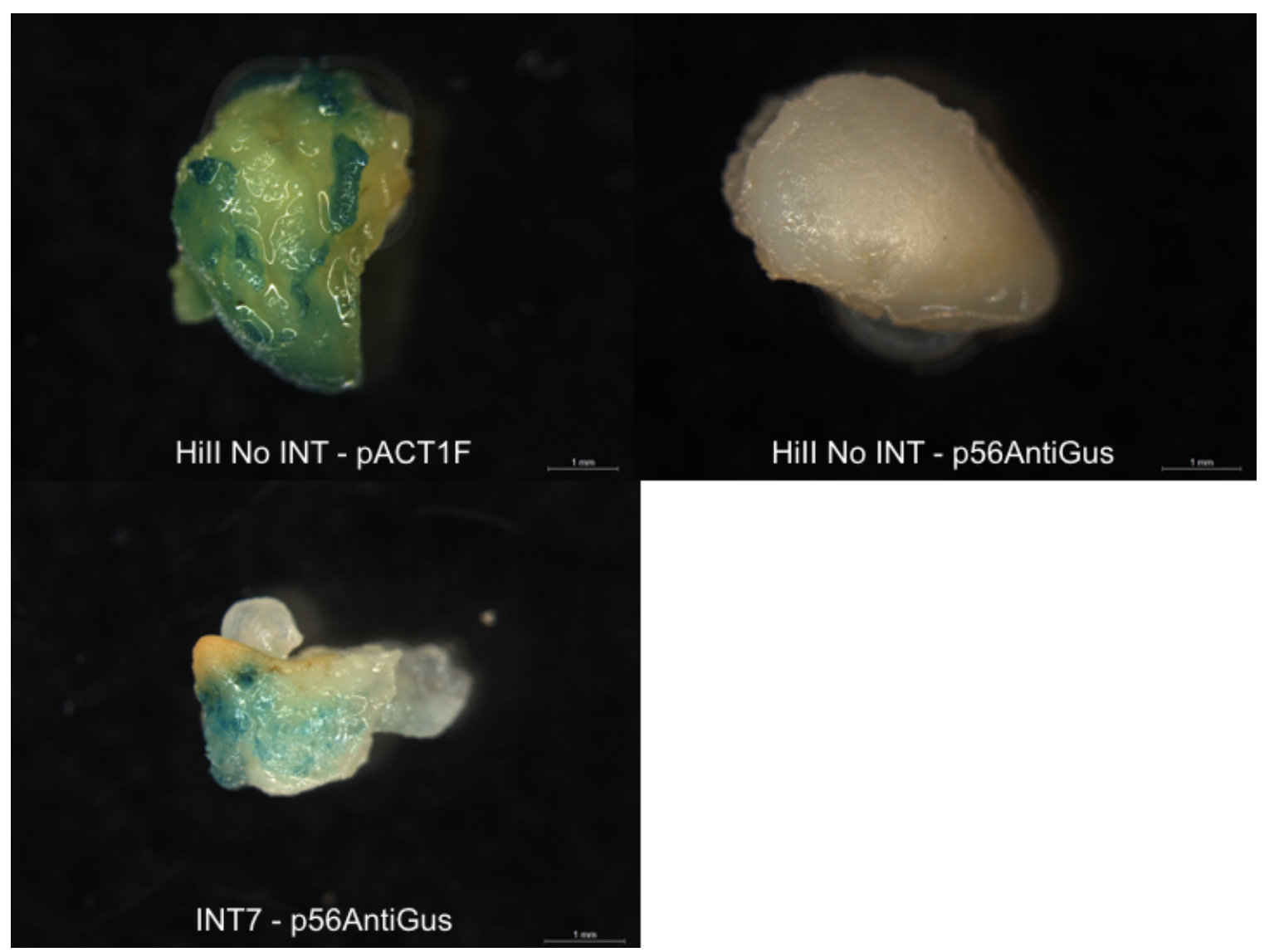

Figure 10 Transgenic Integrase Activity

Bombardment experiments on a transgenic line containing integrase. (Top Left) Positive control with actin-GUS reporter to check bombardment success. (Top Right) Reporter construct bombarded into standard Hi-Il embryos. (Bottom) Reporter construct bombarded into transgenic line INT7 containing ФC31 integrase. 


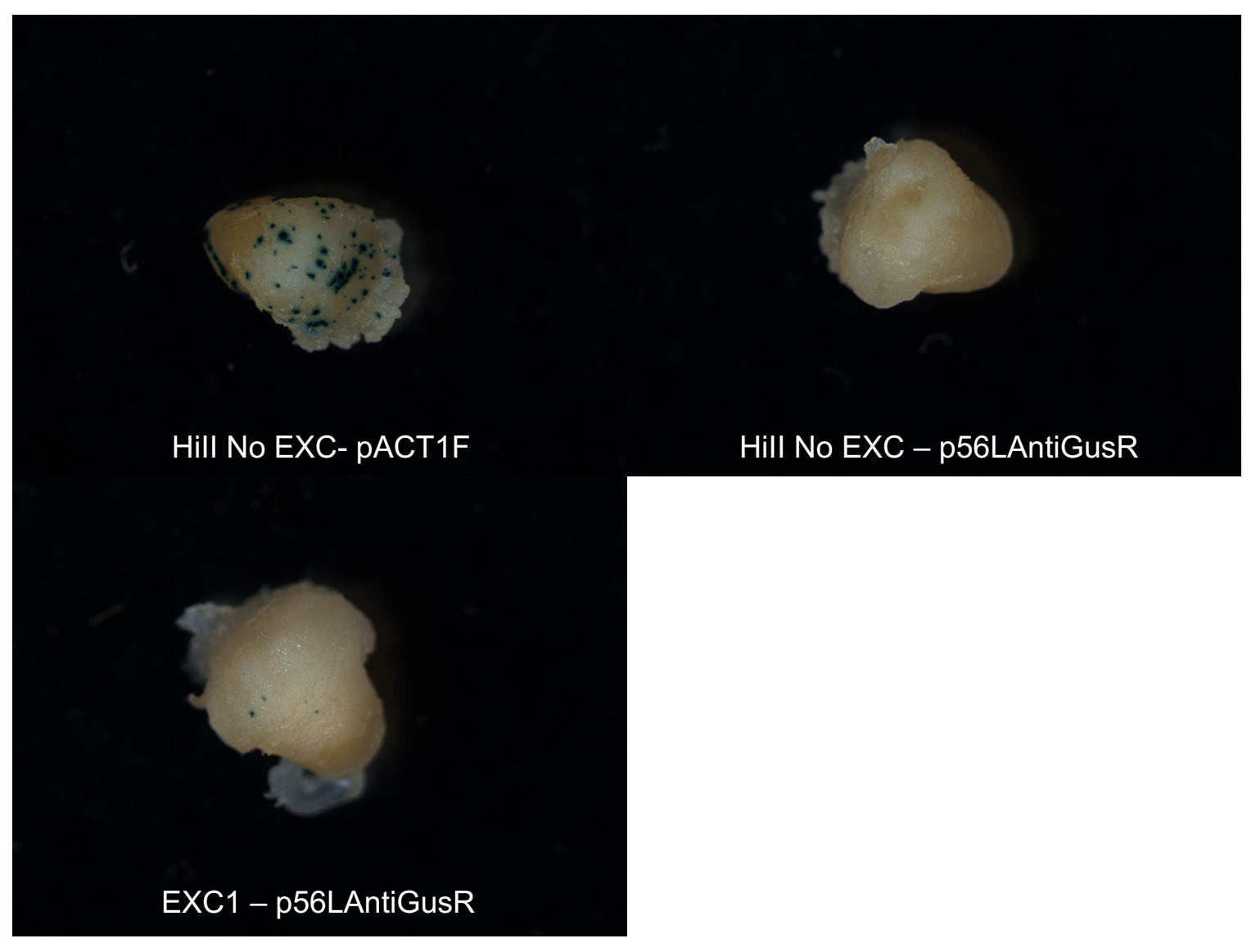

Figure 11 Transgenic Excisionase Activity

Bombardment experiments on transgenic excisionase lines. (Top Left) Positive control with actinGUS reporter construct. (Top Right) Negative control of reporter construct introduced to standard Hi-II embryo. (Bottom) Reporter construct introduced to excisionase transgenic line.

\section{Discussion}

Recombinases have been utilized in plants for decades; however, despite their high use, most research still uses reversible systems. While reversible systems, such as the Cre/lox recombinase system, are efficient for transgene removal, their use for site-specific integration is limited. While this limitation can be overcome through the use of mutant recombination sites, introduction of subsequent transgenes is even more difficult. 
Despite the drawbacks of the currently used recombinase systems, the use of unidirectional systems in plants has been limited. To remedy this problem, the unidirectional recombinase system based on the Streptomyces phage ФC31 been examined in maize. The $\Phi C 31$ integrase and excisionase system improves upon the currently used recombination systems as the recombination sites are altered during recombination. When attB and attP sites are recombined, the sites generated are half attB and half attP. These new sites, attL and attR, are not capable of reverting to their original sequence in the presence of $\Phi C 31$ integrase alone. However, when the recombination directionality factor excisionase is also introduced, which binds to and causes a conformational change in the integrase molecule (Pokhilko et al., 2016), the reverse reaction is allowed to proceed. By utilizing this system for transgene manipulation, site-specific transgene insertion may be possible. Additionally, transgene excision could be easily accomplished by simple crossing to an excisionase containing line.

In this study, both ФС 31 integrase and excisionase were shown to be functional in maize cells. Importantly, the system was functional both when introduced transiently though particle bombardment and when inserted previously into the genome. This is useful for transgenic production, as it suggests that it may be possible to insert transgenes into lines that contain an attachment site, but not the recombinase. As a result, it may be possible to codeliver a transgene and recombinase sequence, but only recover the inserted transgene. Consequently, a step could be saved in transgenic line production as outcrossing to remove the recombinase sequence may not be required. 
While integrase was functional both transiently and when introduced into the genome, there was a noticeable increase in GUS signal when introduced into the integrase transgenic line. This was consistent among all examined embryos, with the integrase containing line showing GUS signal at an amount consistent with or exceeding the actin control. This result is expected as each cell would be expressing the recombinase protein and capable of recombining the incoming GUS transgene. When cobombarded, not only is there a possibility of only one transgene entering the cell, but also due to the large size of the integrase sequence, there is a higher chance of it fracturing during bombardment. If the ФC31 recombinase system is to be utilized for a transformation strategy, this suggests that the most efficient strategy will be to utilize an integrase expressing line. The level of expression shown by the INT7 line, figure10, suggests that the efficiency of the recombinase is quite high.

The amount of GUS signal from excisionase experiments suggested that it was much less efficient than integrase in maize cells. In both transient and transgenic methods, the GUS signal was weak, and in the case of cobombardment was visible in few locations. There are a few explanations for why this might be the case. First, excisionase relies on two proteins being expressed and localized together, and this complex also coming into contact with the introduced reporter construct. Secondly, the two sequences are linked with a P2A peptide, which allows for two peptides to be expressed under a single promoter (Kim et al., 2011). These peptides have not been tested for efficiency in maize, and could be the cause of reduced activity. Lastly, the large size of the 
construct may result in an increased chance of it becoming fractured during the bombardment process, reducing the efficiency.

As the embryos used for this study were heterozygous, it is possible that each recombinase could be more efficient when in a homozygous line due to transgene dosage effects. In the case of excisionase, the presence of more copies of each protein may lead to an increase in complex formation and thus increasing efficiency. For both recombinases, any increase in protein amount has the potential to increase efficiency by increasing the chances the protein and substrate are in the same location. If $\Phi C 31$ integrase is to be used for site-specific integration, it is worth considering the relative size of the recombination sites to the whole genome. In the case of the maize genome, which is over 2.3 gigabases (Schnable et al., 2009), an inserted attP site at $39 \mathrm{bp}$ would make up only $0.000002 \%$ of the genome. For site-specific integration to occur the recombinase will need to locate this recombinase site, as well as a recombination site on an incoming transgene. While the rarity of the recombination site is a concern, expression of the protein in each cell in a homozygous line may improve the chances of success. Whether this is necessary for efficient transformation will need to be tested empirically.

In conclusion, ФC31 integrase and excisionase are functional in maize cells. Due to the importance of maize as an agricultural crop species, and the difficulties in its transformation, this work can lay the framework to a more efficient transformation strategy in the future. 


\section{References}

Albert, H., Dale, E., Lee, E., and Ow, D. (1995). Site-specific integration of DNA into wild-type and mutant lox sites placed in the plant genome. The Plant Journal : For Cell and Molecular Biology 7, 649-659.

Christensen, A.H., Sharrock, R.A., and Quail, P.H. (1992). Maize polyubiquitin genes: structure, thermal perturbation of expression and transcript splicing, and promoter activity following transfer to protoplasts by electroporation. Plant Molecular Biology 18, 675-689.

Colloms, S.D., Merrick, C. a, Olorunniji, F.J., Stark, W.M., Smith, M.C.M., Osbourn, A., Keasling, J.D., and Rosser, S.J. (2013). Rapid metabolic pathway assembly and modification using serine integrase site-specific recombination. Nucleic Acids Research 1-10.

Dale, E., and Ow, D. (1991). Gene transfer with subsequent removal of the selection gene from the host genome. Proceedings of the National Academy of Sciences of the United States of America 88, 10558-10562.

Farruggio, A.P., Chavez, C.L., Mikell, C.L., and Calos, M.P. (2012). Efficient reversal of phiC31 integrase recombination in mammalian cells. Biotechnology Journal 7, 1332-1336.

Gils, M., Marillonnet, S., Werner, S., Grützner, R., Giritch, A., Engler, C., Schachschneider, R., Klimyuk, V., and Gleba, Y. (2008). A novel hybrid seed system for plants. Plant Biotechnology Journal 6, 226-235.

Groth, A.C., Olivares, E.C., Thyagarajan, B., and Calos, M.P. (2000). A phage 
integrase directs efficient site-specific integration in human cells.

Proceedings of the National Academy of Sciences 97, 5995-6000.

Khaleel, T., Younger, E., McEwan, A.R., Varghese, A.S., and Smith, M.C.M.

(2011). A phage protein that binds PhiC31 integrase to switch its directionality. Molecular Microbiology 80, 1450-1463.

Kim, J.H., Lee, S.R., Li, L.H., Park, H.J., Park, J.H., Lee, K.Y., Kim, M.K., Shin, B.A., and Choi, S.Y. (2011). High cleavage efficiency of a 2A peptide derived from porcine teschovirus-1 in human cell lines, zebrafish and mice. PLoS ONE 6, 1-8.

Lutz, K. a., Corneille, S., Azhagiri, A.K., Svab, Z., and Maliga, P. (2004). A novel approach to plastid transformation utilizes the phiC31 phage integrase. The Plant Journal 37, 906-913.

Medberry, S.L., Dale, E., Qin, M., and Ow, D.W. (1995). Intra-chromosomal rearrangements generated by Cre-lox site-specific recombination. Nucleic Acids Research 23, 485-490.

Pokhilko, A., Zhao, J., Ebenh?h, O., Smith, M. ?M., Stark, W.M., and Colloms, S.D. (2016). The mechanism of PhiC31 integrase directionality: experimental analysis and computational modelling. Nucleic Acids Research 44, gkw616.

Rubtsova, M., Kempe, K., Gils, A., Ismagul, A., Weyen, J., and Gils, M. (2008). Expression of active Streptomyces phage phiC31 integrase in transgenic wheat plants. Plant Cell Reports 27, 1821-1831.

Schnable, P.S., Ware, D., Fulton, R.S., Stein, J.C., Wei, F., Pasternak, S., Liang, 
C., Zhang, J., Fulton, L., Graves, T.A., et al. (2009). The B73 Maize

Genome: Complexity, Diversity, and Dynamics. Science 326, 1112-1115.

Smith, M.C.A., Till, R., Brady, K., Soultanas, P., Thorpe, H., and Smith, M.C.M. (2004). Synapsis and DNA cleavage in phiC31 integrase-mediated sitespecific recombination. Nucleic Acids Research 32, 2607-2617.

Songstad, D.D., Armstrong, C.L., Petersen, W.L., Hairston, B., and Hinchee, M. a W. (1996). Production of Transgenic Maize Plants and Progeny by Bombardment of Hi-II Immature Embryos. In Vitro Cellular \& Developmental Biology - Plant 32, 179-183.

Thorpe, H.M., and Smith, M.C. (1998). In vitro site-specific integration of bacteriophage DNA catalyzed by a recombinase of the resolvase/invertase family. Proceedings of the National Academy of Sciences of the United States of America 95, 5505-5510.

Vega, J.M., Yu, W., Kennon, A.R., Chen, X., and Zhang, Z.J. (2008). Improvement of Agrobacterium-mediated transformation in Hi-II maize (Zea mays) using standard binary vectors. Plant Cell Reports 27, 297305.

Vergunst, A.C., Jansen, L.E.T., and Hooykaas, P.J.J. (1998). Site-specific integration of Agrobacterium T-DNA in Arabidopsis thaliana mediated by Cre recombinase. Nucleic Acids Research 26, 2729-2734. 


\title{
Chapter 3 Evidence for Site-Specific Transgene Integration
}

\section{Using ФC31 Integrase}

\begin{abstract}
:
Transgene integration occurs through the use of either particle bombardment or Agrobacterium tumefaciens transformation. In each case, the insertion site of the introduced transgene relies on a random integration process. As a result, transgenes are often linked to undesirable traits in the transformation line and introducing multiple transgenes results in a dispersed distribution throughout the genome. Maintaining these multiple transgenes in the future requires complex breeding strategies and intense screening. Many of these complications can be overcome with site-specific transgene integration, which allows for a precise insertion site to be chosen for integration. In this study, the recombinase $\Phi C 31$ integrase is utilized for site-specific transgene insertion. A target site is first introduced into the genome and a reporter construct is targeted to that site, turning on GUS expression. In Arabidopsis thaliana there was no evidence of site-specific integration through floral dip. However, in maize the results indicate that site-specific integration may be possible.
\end{abstract}

\section{Introduction}

Transgenic technology in maize has, since its inception, relied on either particle bombardment or Agrobacterium tumefaciens transformation. Particle bombardment introduces transgenes by shooting them at high velocity into cells 
on gold particle micro carriers. Particles are able to penetrate the cell wall and membranes, allowing them to enter the nucleus and fractionate DNA molecules as well (Christou, 1991). The transgene integration mechanism is for the most part random. Provided that no vital genes were mutated or lost during the process, the incoming transgene can be located anywhere throughout the genome. Agrobacterium tumefaciens transformation relies on horizontal gene transfer for transgene integration. Transgenes are first cloned into the Agrobacterium Ti plasmid before the bacterium is allowed to infect maize embryos. While there are studies that have suggested that transgene integration with Agrobacterium is nonrandom (Brunaud et al., 2002; Koncz et al., 1989; Sallaud et al., 2004), most of these studies have relied on an active selection gene for transgene recovery. As a result, transgenes inserted into regions with limited transcription will be selected against during regeneration. Indeed, when insertion sites were examined without a selection regime, the Agrobacterium insertion strategy was also found to be random (Kim and Gelvin, 2007).

Random transgene insertion can cause many problems for downstream applications. The maize line Hi-II, the predominantly used line for transformation, is of little agronomic importance, requiring transgenes to be moved into more important lines after regeneration. Due to random integration, transgenes may be linked to unfavorable alleles that lead to difficulties in introgression. Additionally, if a polygenic trait is to be introduced, or additional traits added to an already existing transgenic line, multiple insertion locations will now exist. As a result, segregation of the traits will occur during meiosis, leading to a low probability of 
the traits being maintained in one plant. As the number of transgenes introduced increases, this complexity of the breeding strategy required to maintain them increases as well.

In Arabidopsis thaliana, where the floral dip procedure can be utilized (Clough and Bent, 1998), the transformation process is relatively easy to carry out. However, as the same Agrobacterium integration mechanism is utilized, transgenes are still scattered randomly through the genome. As a result, the same issues exist in Arabidopsis as in maize, and any organism that utilizes a random transgene integration strategy. In such an approach, it is difficult to maintain multiple transgenes; native genes can be disrupted; and transgenes can have varying expression patterns.

The problems with random integration can be alleviated by using a sitespecific transgene insertion (SSTI) strategy. In SSTI, a transgene can be targeted to a pre-determined location in the genome. As a result, transgenes can be placed into genomic locations that do not interfere with native genes and allow for good expression. If designed correctly, subsequent transgenes can be targeted to the same locus creating a linkage group. By having a suite of transgenes linked, subsequent crossing schemes to agronomically important lines would require much less effort than current strategies. If SSTI is combined with minichromosome technology (reviewed in Graham et al., 2015), transgenes could be targeted outside of the main plant genome, alleviating any problems of linkage completely. 
The most widely used recombination system for SSTI in plants is the Cre/lox system from the phage $\mathrm{P} 1$. Cre recombinase recognizes $34 \mathrm{bp}$ lox sites for recombination and, depending on the orientation of the sites, can result in insertions, deletions, or inversions in plant cells (Dale and Ow, 1991; Medberry et al., 1995; Vergunst et al., 1998). After recombination, however, lox sites are left intact, allowing for further recombination between these sites. When used for SSTI, this means that the newly inserted transgene will be flanked by lox sites and can be excised by the same recombinase protein. This issue can be overcome by utilizing mutant lox sites for recombination, which leave a mutant and wild-type lox site flanking a transgene after insertion (Albert et al., 1995). Unfortunately, with this strategy subsequent transgenes would require integration with wild-type lox sites.

Another issue to be overcome with the Cre/lox system is modifying a transgene once integrated into the genome. Subsequent removal of marker genes is not possible with Cre recombinase alone, so other recombinase systems must be employed. Previous studies have utilized the FLP/frt system or $\mathrm{R} / R S$ for removal of marker genes in plants (Srivastava and Ow, 2004). In this strategy, a transgene is introduced with one recombinase that contains recombination sites for another. Following transformation, the alternate recombinase is introduced for marker excision. While this strategy is successful in marker excision, subsequent transgene integration is still difficult as recombination sites are still present after recombination. 
Many of the problems with current SSTI strategies can be alleviated by utilizing unidirectional recombinases. Unidirectional recombinases, such as ФC31 and BxB1 (Groth et al., 2000; Keravala et al., 2006), are capable of the same excision, integration, and inversion reactions as Cre/lox, but recombine non-identical recombination sites. The result of these recombinations are sites that are different in sequence from the original sites, and are thus not recognized by the recombinase following recombination. Consequently, the reaction is unidirectional in nature, which would allow for a more efficient SSTI system. By combining unidirectional and bidirectional recombinases, a system can be devised that allows for site-specific insertion, marker excision, and subsequent transgene insertion for transgene stacking (Hou et al., 2014; Ow, 2011).

It should also be noted that by utilizing a SSTI system, it may be possible to improve transformation efficiency. In traditional transformation systems, transgene integration relies on native machinery integrating the transgene into regions of high transcription. Additionally, the strategy relies on the transformed cells producing enough resistance protein to make it through selection and regeneration. As previously mentioned, however, insertions into areas of low transcription or necessary genes will prevent transformed cells from developing into full transgenic plants. In practice, transformed cells may also be lost if located in poorly developing callus sectors as callus development is not uniform. Transformed cells can also be lost if the surrounding sector is all killed by a selection agent, preventing transformed cells from obtaining nutrients from the growth media. By utilizing a SSTI strategy, it may be possible to increase the 
occurrence of transformed cells during transformation. First, by choosing where a transgene is inserted in the genome, each transformation insertion will result in resistance protein expression, increasing the number of recoverable events. Additionally, if the insertion target and recombination protein is expressed in every cell, each occurrence of the transgene in the nucleus should result in integration. When using particle bombardment, this could greatly increase transformation efficiency. When transient expression is examined following particle bombardment (Oard et al., 1990), multiple signals can be observed. Compared to the typical $5-10 \%$ transformation efficiency across every embryo, it is clear that particle bombardment consistently delivers DNA into cells; however, it is rarely integrated into the genome. Expression of a recombinase protein concurrent with a target site for integration should allow for a higher number of integrations from particle bombardment.

Both Cre recombinase (Zhang et al., 2003) and ФC31 integrase activity (See Chapter 2) have been demonstrated in maize, and these were chosen as the basis for SSTI in this project (Figure 12). ФC31 integrase recognizes attP and attB sites for recombination in a unidirectional fashion (see chapter 2). To utilize this system for integration, a recombination site must first be introduced into the maize genome. As a result, the target site must be inserted into maize using the traditional random integration method. Insertion sites can then be screened following transformation and analyzed for expression. The target site line is then crossed to a Cre containing line to remove the resistance gene. The target site following Cre excision will then consist of an integrase recombination site behind 
a promoter. By designing the system in this manner, a new resistance protein can be introduced into the integrase target site that contains no promoter. As a result, only successful recombination will result in gene expression. Additionally, as the same genomic location and promoter were previously used for transformation of the target site, it can be assumed that any recombinations into the target will result in sufficient resistant protein for transformation.

In this study, SSTI is demonstrated using a GUS reporter. An insertion site target is inserted into the maize genome, reduced, and targeted by a promoterless GUS construct. Examination of transformed embryos that express the GUS protein show a proof-of-concept that SSTI is possible in maize. 
A.
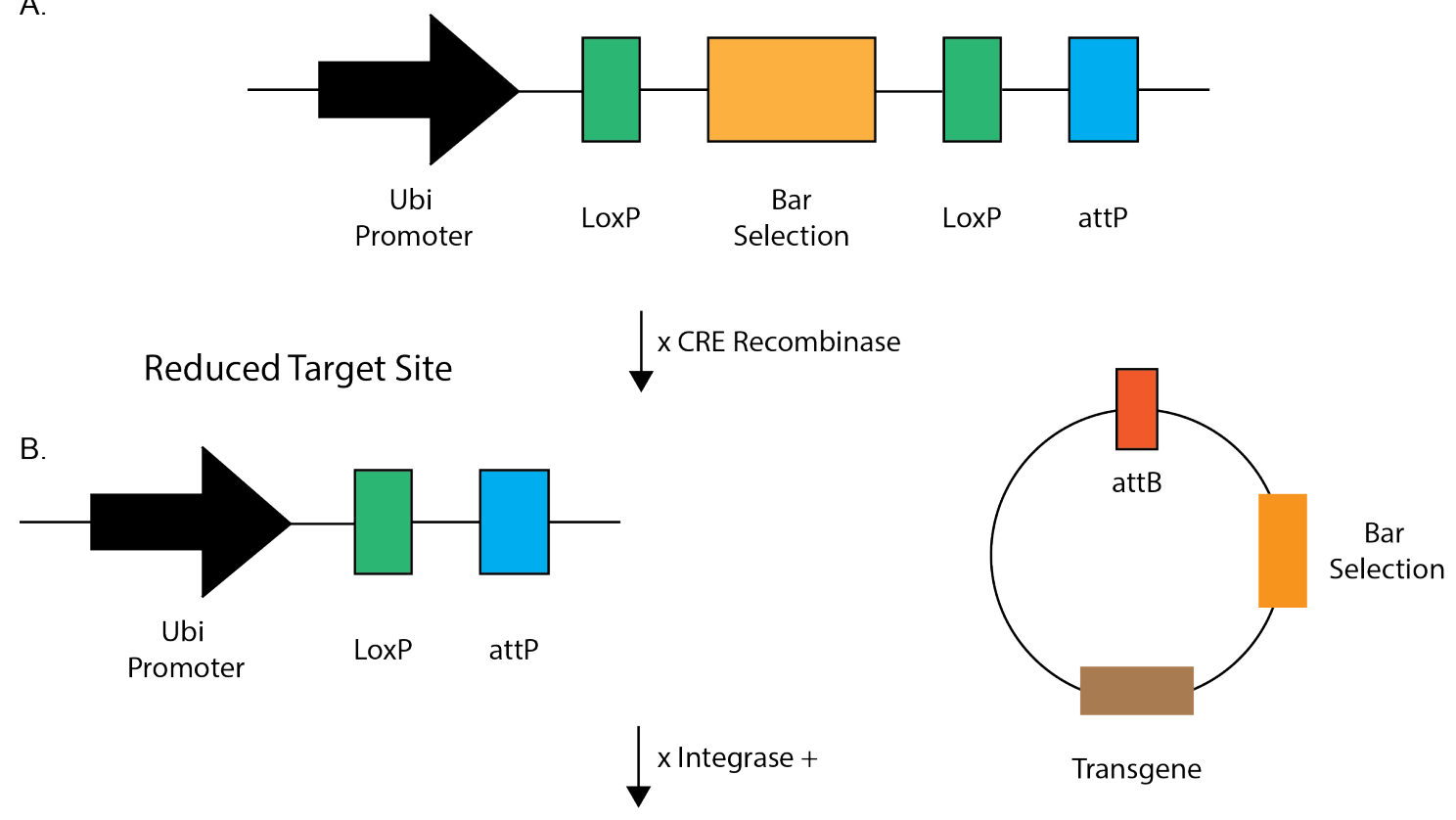

C.

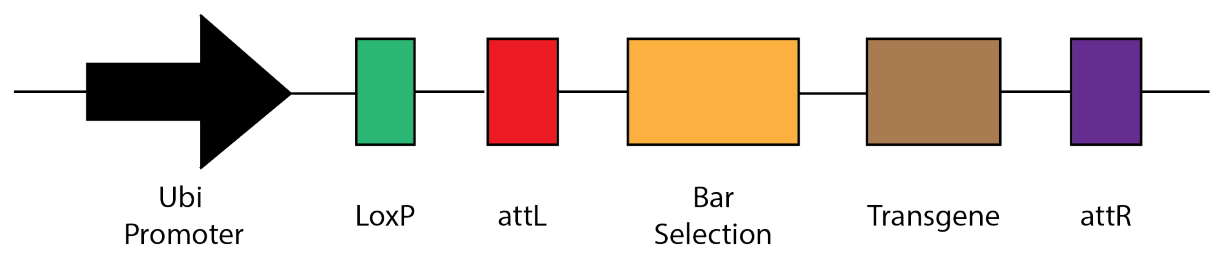

Inserted Transgene with Selection

Figure 12 SSTI Scheme

(A) A target site construct is designed that consists of a ubiquitin promoter driving the selectable marker bialaphos (Bar) that is flanked by loxP sites and followed by the $\Phi \mathrm{C} 31$ integrase recombination site attP. (B) After integration of the target site construct, it is crossed by Cre recombinase to remove the selectable marker. Following marker excision, the attP recombination site is directly behind the ubiquitin promoter, which can be used in subsequent insertion. (C) The reduced target site is used for transgene insertion. The target site construct can either be crossed to an integrase containing line, or integrase can be cobombarded with the incoming transgene. The incoming transgene consists of the recombination site attB followed by a promoterless selection gene followed by the new gene of interest. Upon recombination, the selection gene will be inserted behind the ubiquitin promoter and transcribed, allowing for selection during transformation. 


\section{Materials and Methods}

\section{Target Site Design and Construction}

A construct was designed to serve as a target for SSTI. The construct, hereafter referred to as PPZPGLP (Figure 13), consists of the maize polyubiquitin promoter (Christensen and Quail, 1996) followed by a bialaphos (bar) resistance gene (Thompson et al., 1987). The bar gene is flanked by loxP sites in direct orientation, which will cause excision in the presence of Cre recombinase. The selection cassette is followed by an attP site for future transgene targeting. The minimal 39 bp recombination site (Groth et al., 2000) was chosen, as the small size was more accommodating for cloning manipulations.

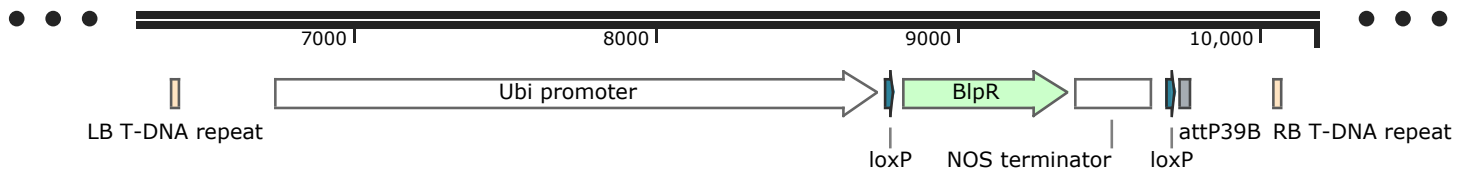

\section{PPZPGLP
10,187 bp}

Figure 13 Insertion Site Construct pPZPGLP

The insertion site construct pPZPGLP to be used for transgene targeting in maize. The maize polyubiquitin1 promoter drives expression of a removable bialaphos resistance gene. The bialaphos resistance gene is flanked by loxP sites in direct orientation. The resistance cassette is followed by an attP site that will be driven by the ubiquitin promoter once the bialaphos resistance gene cassette has been removed by Cre recombinase.

pPZPGLP was constructed by digesting plasmid pPZP201:004_GZUS, which consists of the cassette Ubi-IoxP-Bar-Nos-loxP- 
Nos-SPMTIR in an Agrobacterium competent transformation vector, with EcoRV and Pacl restriction enzymes to remove the loxP-Nos-SPMTIR sequence. Into this digested plasmid a synthesized loxP-attP cassette was inserted to create plasmid pPZPGLP. Transformation of pPZPGLP was performed using Agrobacterium tumefaciens line EHA105 following a standard Agrobacterium transformation protocol (Vega et al., 2008b).

\section{Reporter Construct Design and Construction}

A reporter construct was designed to serve as a proof-of-concept to test the SSTI strategy in maize. The construct pB34GUSNos (Figure 14), consists of a promoterless GUS reporter gene following the minimal attB site. The GUS reporter will only be active if inserted behind a functional promoter. The attB - GUS - NOS cassette of pB34GUSNos was synthesized and cloned (GeneWiz) into pUC57 to create the reporter construct.

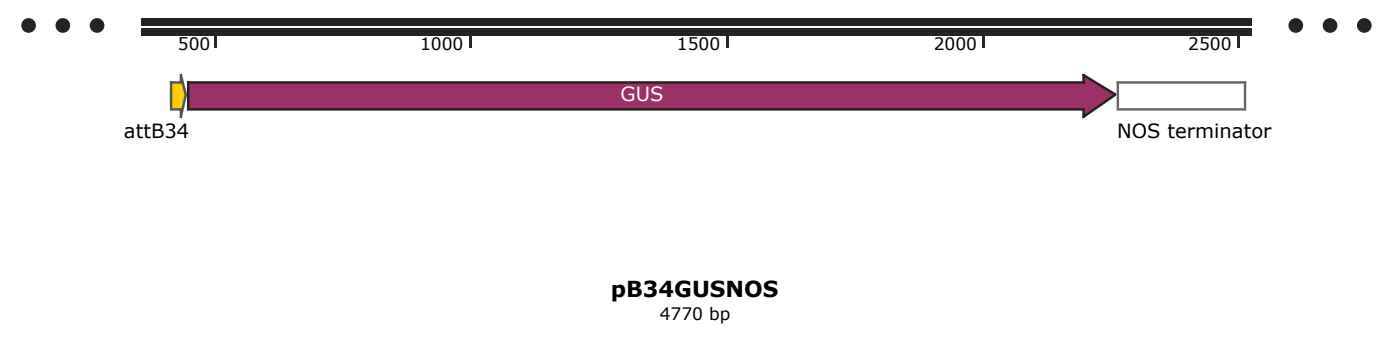

Figure 14 Reporter Construct pB34GUSNos

Reporter construct to test SSTI in maize. The construct consists of the recombination site attB followed by GUS protein sequence and terminator. 


\section{Particle Bombardment}

Test constructs were introduced to maize embryos that were extracted 1012 days after pollination. Embryos, which measured $1.5-2 \mathrm{~mm}$ in length were first placed on N6 media for 3-5 days (Songstad et al., 1996) to initiate callus formation and moved to osmotic media 4 hours prior to bombardment. About 1 $\mu \mathrm{g}$ of DNA was loaded onto $0.6 \mu \mathrm{M}$ gold particles (Bio-Rad) for bombardment. In the case of cobombardment $1 \mu \mathrm{g}$ of each construct was loaded, irrespective of construct size. Bombardment was carried out in a Biolistic PDS-1000/He Particle Delivery System (Bio-Rad) with 650 psi rupture disks. One hour after bombardment embryos were moved to N6 media.

\section{GUS Staining}

Embryos were allowed to rest for 3-5 days before GUS staining. The BetaGlucuronidase Reporter Gene Staining Kit (Sigma-Aldrich) was used for staining, omitting the fixation step. After 24-72 hours of staining, a Leica M205F stereoscope was used to image results.

\section{Fluorescent In-Situ Hybridization (FISH)}

Chromosome spreads were produced and hybridized following the standard Birchler lab protocol (McCaw et al., 2016a, 2016b). Centromere (CentC) and TAG probes were ordered as fluorescently labeled oligonucleotides. Single-gene probes were produced with PCR amplified DNA fragments that were later nick-translated. Images were taken with an Olympus BX61 fluorescent 
microscope.

\section{Results}

\section{Target Site Insertion and Manipulation}

The construct pPZPGLP was introduced through Agrobacterium tumefaciens transformation into maize line Hi-II. Two events (GLP1 and GLP2) were recovered after transformation, which were PCR amplified and sequenced to ensure construct integrity. Root tips were obtained from developing plants and hybridized with a probe specific to the pPZPGLP locus (figure 15 and 16). FISH results show that event GLP1 inserted a target site on the long-arm of chromosome 9, and GLP2 inserted two target sites, one on the long-arm of chromosome 9 and one on the short arm of chromosome 5 . 


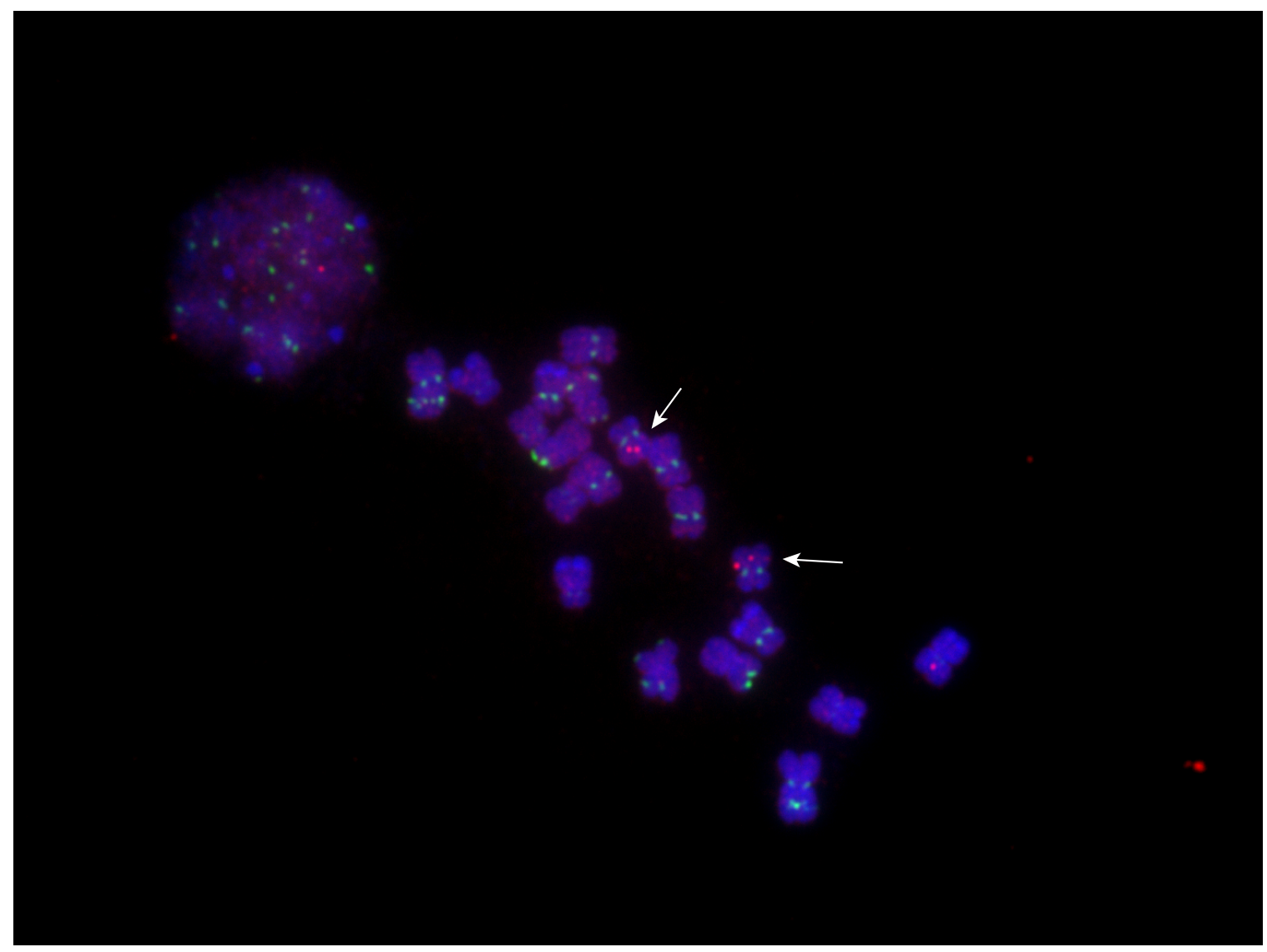

Figure 15 FISH Image of Homozygous GLP1 Plant

FISH image of homozygous GLP1 plant. Centromere and TAG signals can be observed in green and single-gene probe to PPZPGLP labeled in red. Insertion site can be observed on the long arm of chromosome 9. 


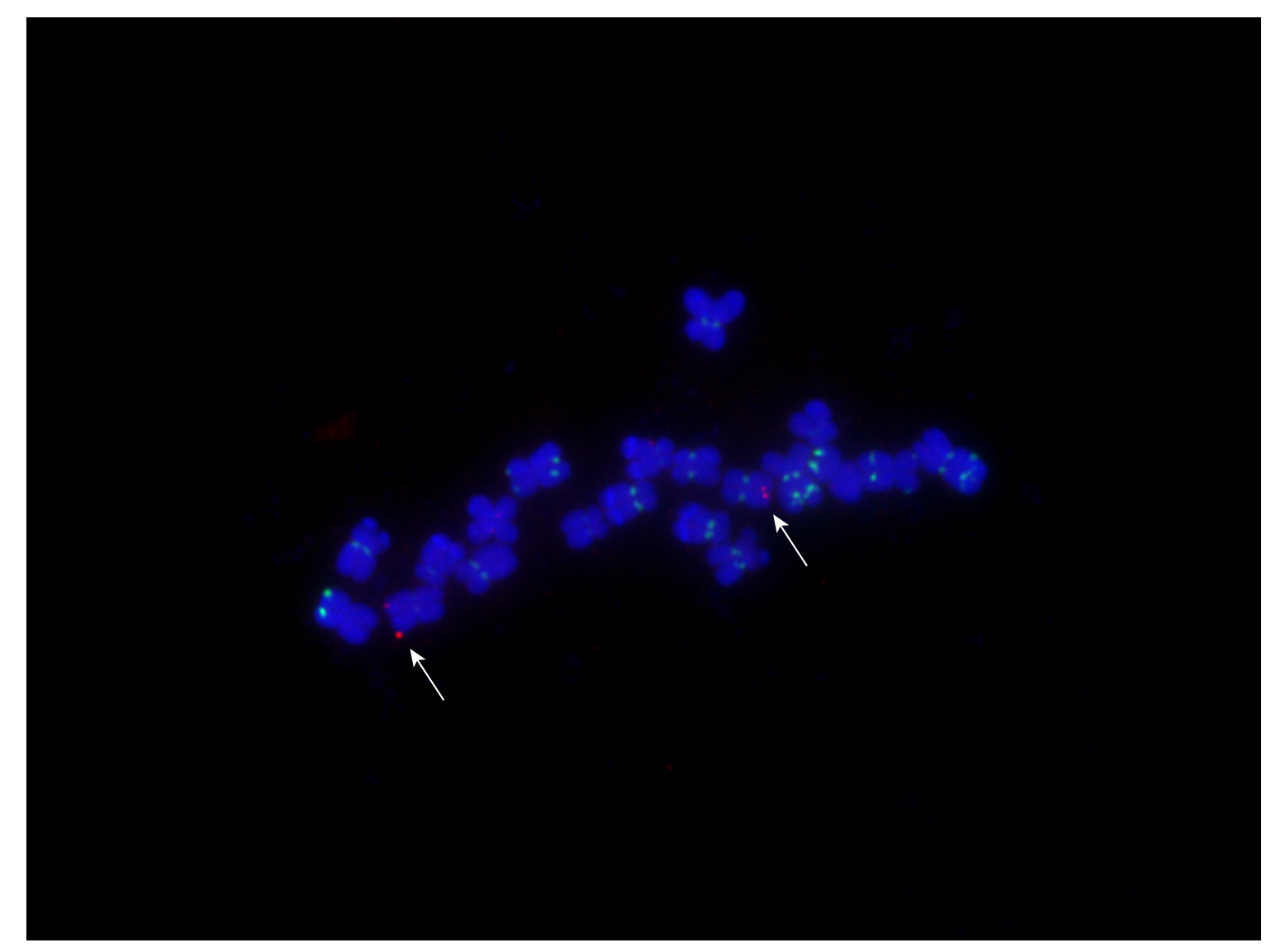

Figure 16 FISH Image of GLP2 Plant

FISH image of GLP2 plant with centromere and TAG repeats labeled in green. The single-gene probe of pPZPGLP can be seen in red localizing to two insertion sites, one on the long arm of chromosome 9 and one on the short arm of chromosome 5.

GLP events were crossed to a Cre recombinase containing line in the greenhouse following transformation and regeneration to remove bar selection. The resultant seed from these plants was then planted and screened via PCR. Using primers that amplified across the bialaphos resistance gene, recombinations could be easily screened (figure 17). The result of the screen showed that any time that the GLP locus was present with Cre recombinase the 
bialaphos sequence was removed. This suggests that excision with Cre recombinase is highly efficient at the genomic locus of the target sites. To further confirm the recombination reaction had proceeded as expected, and the attP site

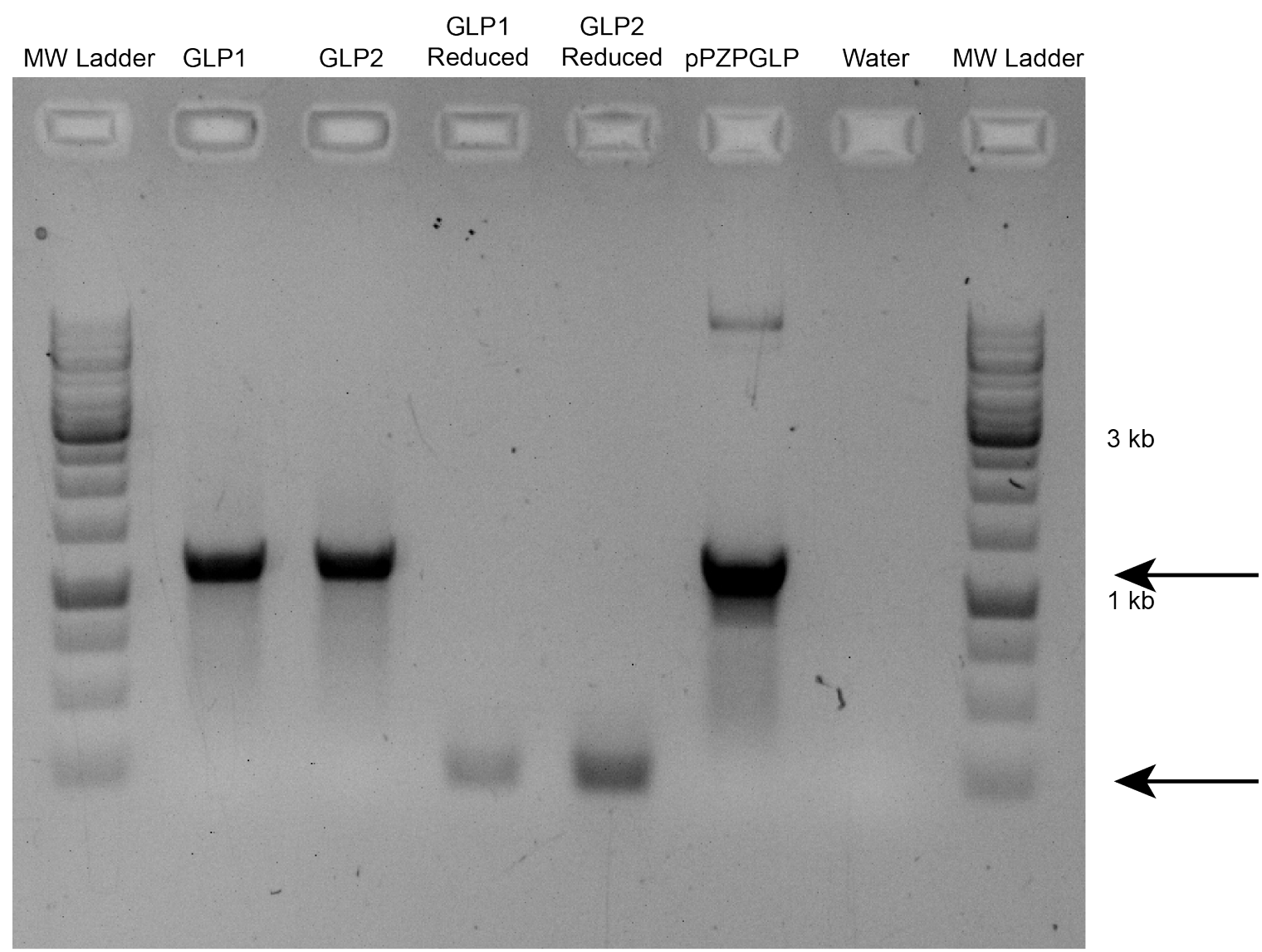

Figure 17 Representative Gel Image of GLP PCR

Demonstration of reduction of target site construct after cross to Cre recombinase. Primers, which amplify from the end of the ubiquitin promoter to after the attP site, are used for screening of recombination events. Upper bands represent the full GLP target site (1170 bp), lower bands show the reduction in size after marker excision (236 bp). Band size is compared to the GeneRuler $1 \mathrm{~kb}$ ladder.

was still intact, PCR product was Sanger Sequenced (Figure 18). As the 
recombination had proceeded as expected for both GLP1 and GLP2, these lines were deemed acceptable for SSTI.

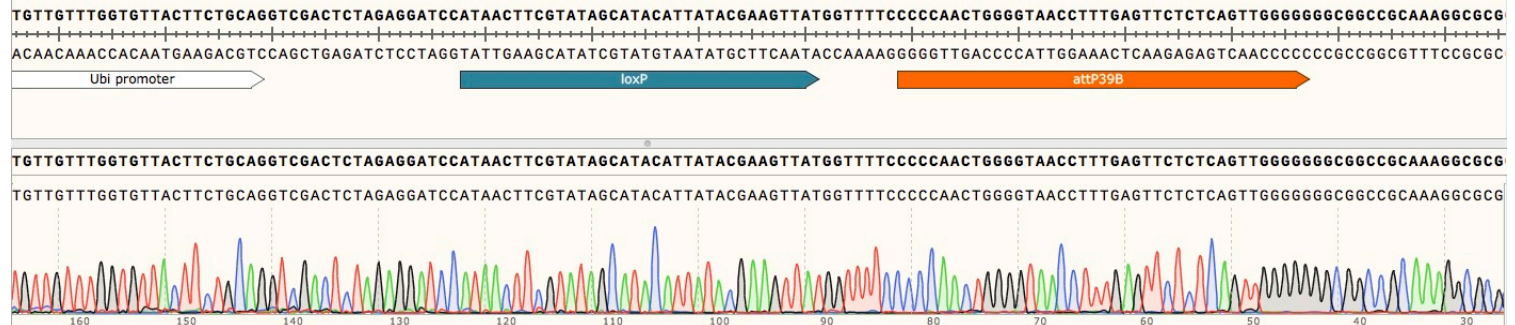

Figure 18 Sanger Sequencing of Reduced GLP

Alignment of sanger sequencing to expected reduced GLP sequence demonstrating that excision proceeded as expected.

\section{Site-Specific Integration into GLP}

Previous work on ФС31 integrase activity (chapter 2) suggested higher activity when the recombinase was already integrated into the genome. However, from a practical standpoint, being able to perform SSTI without the need for an additional integrated transgene would be beneficial. To determine if SSTI was possible with $\Phi С 31$ integrase inserted into the genome and when introduced through cobombardment, reduced GLP plants were selfed and crossed to INT7 (chapter 2). Ears from resultant plants were used for transformation with the reporter construct. As a positive control for the bombardment procedure, nontransgenic Hi-II embryos were bombarded with pACT1F, a plasmid containing a GUS reporter under the constitutive actin promoter. To ensure that the reporter plasmid would not confer GUS protein on its own, and required ФC31 integrase for activity, the reporter pB34GUSNos was introduced to GLP embryos not 
containing $\Phi C 31$ integrase as a negative control. To test for SSTI activity via cobombardment, GLP embryos without $\Phi C 31$ integrase were bombarded with both pB34GUSNos and pTFPhiC31 (ФC31 integrase under the maize ubiquitin promoter). Embryos that resulted from the cross of GLP and INT7 plants were bombarded with the reporter construct only. The results of the bombardment experiments can be observed in figures 19 and 20.

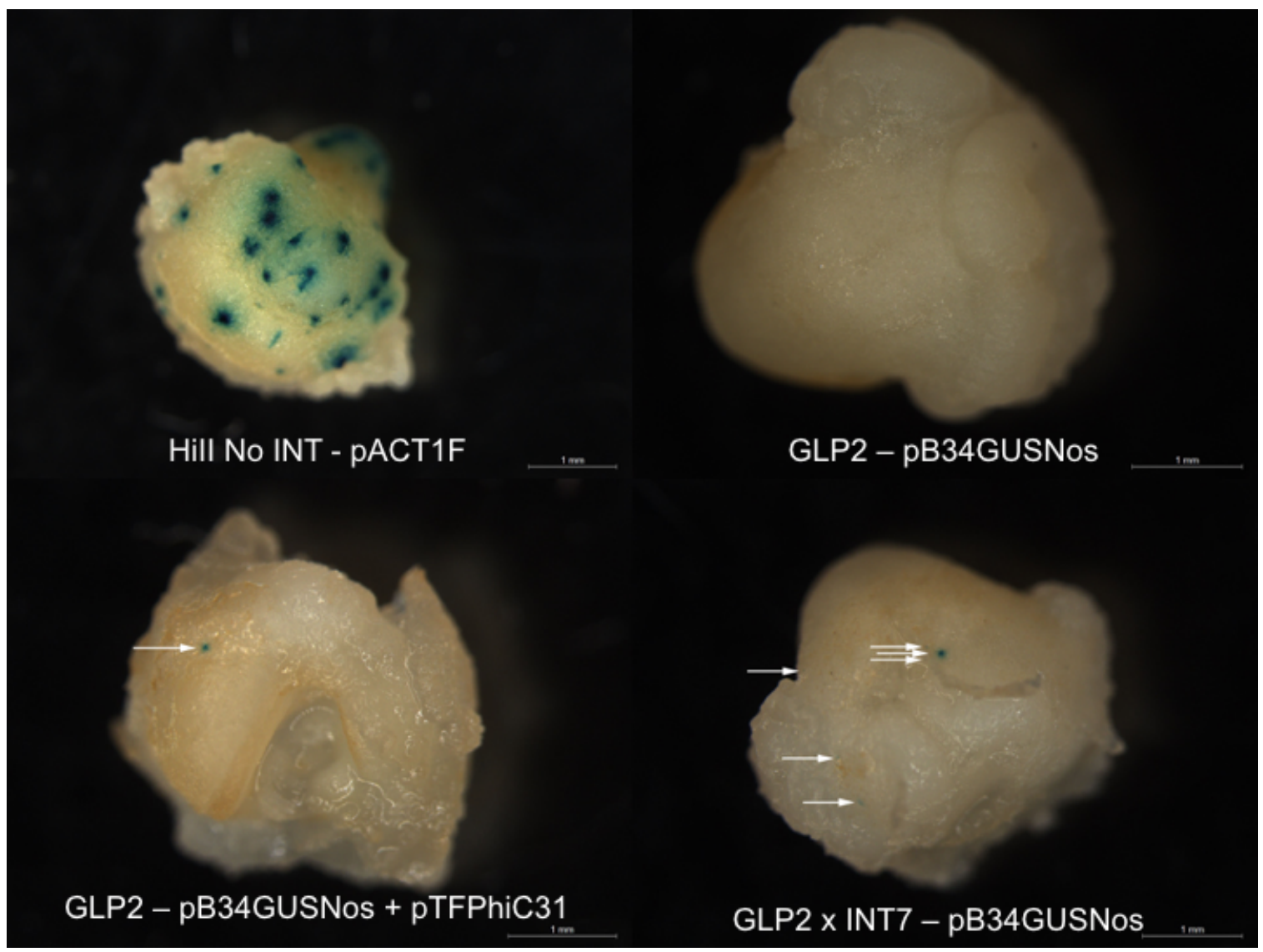

Figure 19 Demonstration of SSTI in Maize with Reporter Plasmid

Bombardment of reporter construct to demonstrate site-specific integration in maize. Embryos shown demonstrate average results across all embryos screened. (Top Left) Positive control of plasmid pACT1F which contains a GUS reporter under an actin promoter. (Top Right) Negative control of reporter construct introduced to GLP2 line showing no GUS expression without 
integrase present. (Bottom Left) Cobombardment of reporter construct with integrase. GUS expression, arrow, demonstrating integration of reporter construct into target site with cobombardment. (Bottom Right) Bombardment of reporter construct into embryo containing both target site and integrase. GUS expression, arrows, shows integration of the reporter construct into the target site. A comparison between the bottom left and right panels shows a higher efficiency of integration with an already introduced integrase protein.

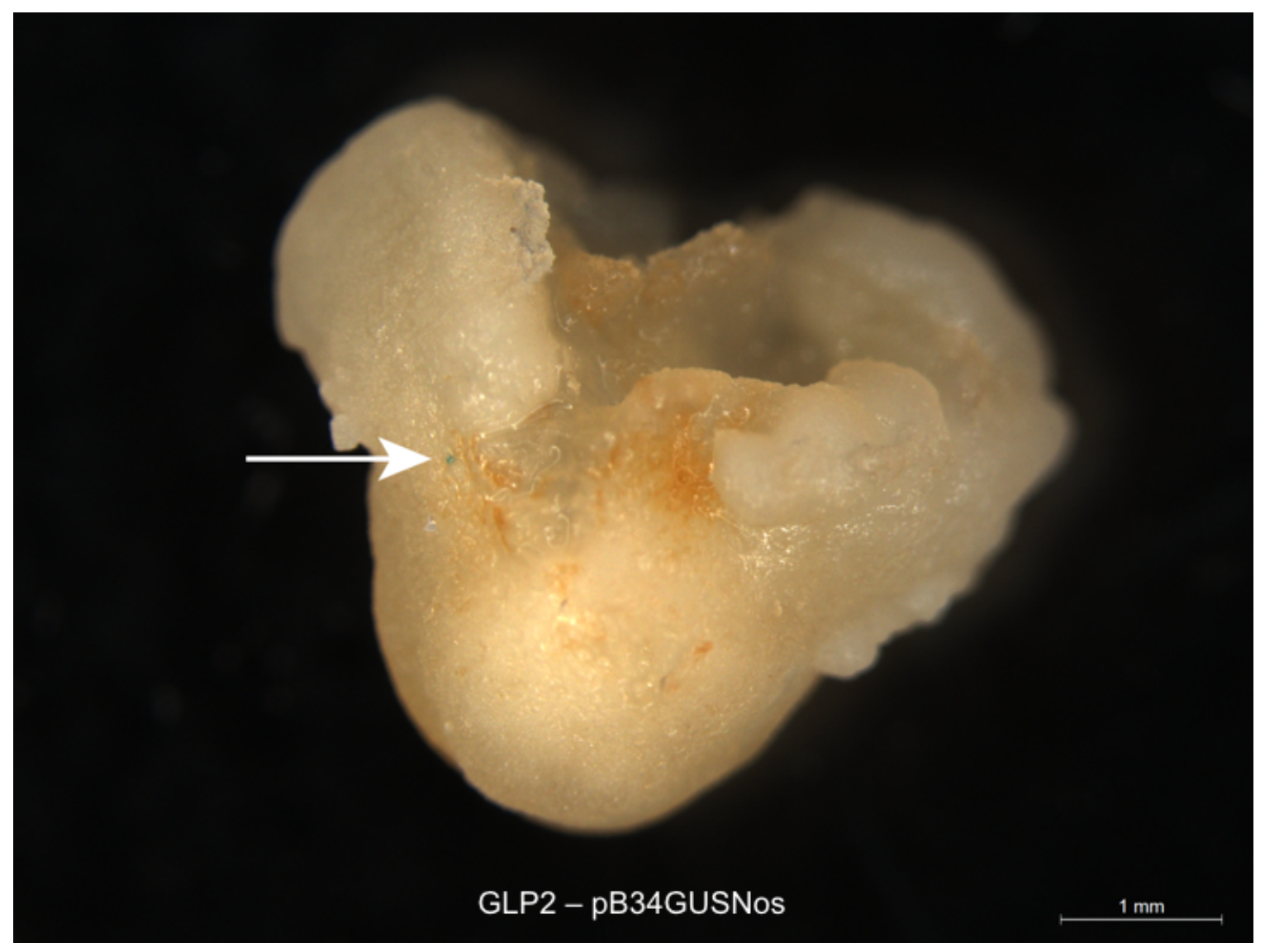

Figure 20 Negative Control Embryo Showing GUS Signal

GUS expression on a negative control embryo. Across the approximately 200 embryos screened, only a single embryo demonstrated any evidence of GUS signal. GUS expression (arrow) can be observed as a single blue dot. 
The embryos shown are only a representative sample of those bombarded. As the embryos bombarded were crosses of heterozygous plants, each embryo was not the same genotype from the same harvested ear. As a result, it is difficult to make observations about transformation efficiency with this trial. To make a true observation about efficiency a uniform genotype of embryos must be compared.

\section{SSTI in Arabidopsis}

The same SSTI strategy was also attempted in Arabidopsis thaliana. Transgenic lines were produced that contained a target site, Cre recombinase, and $\Phi C 31$ integrase. These lines were screened to ensure that the transgenes were intact, and crosses made to reduce the target site construct. Following target site reduction, the reduced site was crossed to a $\Phi C 31$ integrase containing line. Previous studies have shown that during integration Agrobacterium plasmids are circularized (Singer et al., 2012), a requirement for SSTI with $\Phi C 31$ integrase. To take advantage of this property of T-DNA integration, plants genotyped to contain both the reduced target site and ФC31 integrase were dipped with a hygromycin integration construct (figure 21).
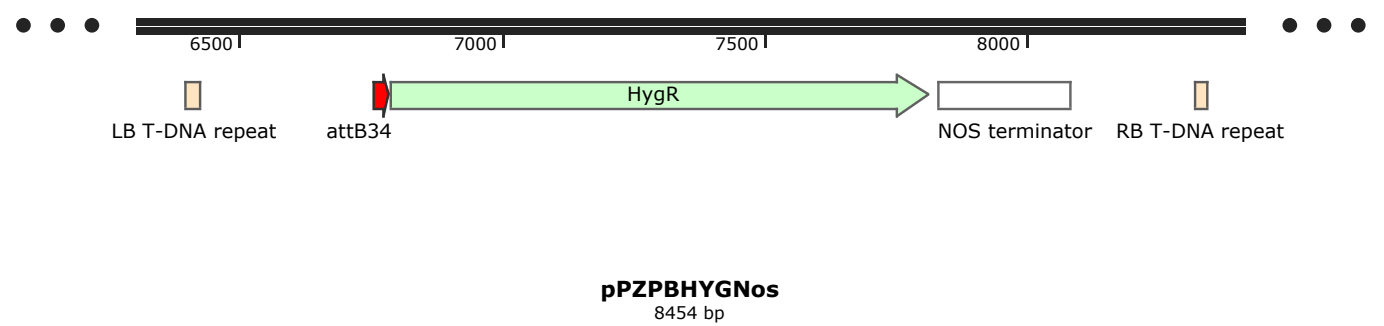

Figure 21 Hygromycin Integration Construct pPZPBHYGNos 
Integration construct to demonstrate SSTI with Arabidopsis through floral dip. Integration into the target site will result in expression of hygromycin resistance protein.

To test for integration, dipped seed was plated on growth medium containing hygromycin. After 2 weeks of growth plates were examined for resistant plants which would be capable of growing in the presence of the selection agent. Wild-type Col plants were not capable of growing in the presence of hygromycin, demonstrating that the selection agent was active in the growth media. Plants dipped at the same time as the experimental group with a 35S-Hygromycin construct could be found growing in the presence of hygromycin, suggesting that the dip procedure was successful. However, no plants from the experimental group could be found that were resistant to hygromycin selection. This was the case for multiple dipped plants with different integration target sites and different $\Phi \mathrm{C} 31$ integrase events.

While studies suggest that T-DNA molecules form circles during integration (Singer et al., 2012), it is unclear at what point in the process this occurs, and was not investigated during the dipping process. As a result, it was thought that relying on circularization during T-DNA integration may have lead to a lack of recombination events. To remedy this problem, reduced target site plants with $\Phi C 31$ integrase were crossed to plants containing Cre recombinase. Plants containing all three transgenes were then dipped with an integration construct that would be circularized in the presence of Cre recombinase (figure 
22). Mutant lox sites were used to flank the hygromycin resistance gene, resulting in a unidirectional circularization reaction.

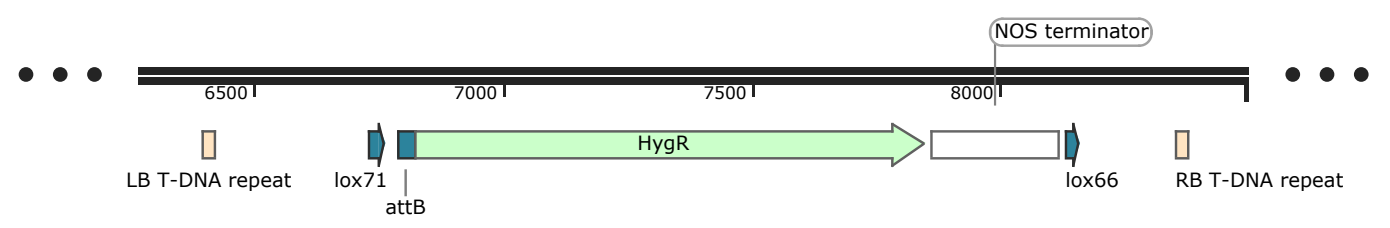

pPZPBHYGNosLox7166

$8490 \mathrm{bp}$

Figure 22 Hygromycin Integration Construct with Lox Sites

Integration construct for testing SSTI in Arabidopsis that will be circularized in the presence of Cre recombinase. Once in the nucleus, Cre recombinase will recognize and recombine the mutant lox71/66 sites, causing circularization. As mutant sites are used, circularization cannot be reversed by Cre recombinase. After circularization, $\Phi C 31$ will recognize the attB recombination site, inserting the construct into the target site allowing transcription of the resistance protein.

To examine if the dip with the new strategy was successful, about 9000 seeds from two different integrase lines were plated on hygromycin plates (figure 23). After two weeks, resistant plants could be observed on the positive control plates, but no transformed plants could be observed on the negative control or experimental plates. Counts of regenerated seeds were made to determine that the positive control experiment had a transformation efficiency of about $4 \%$. As about 9000 seeds were screened from each integrase line, around 360 transgenic plants could be expected for each if the efficiency was similar to the positive control. This result suggests that the mechanism utilized for transformation of Arabidiopsis may not be conducive to SSTI with site-specific recombinases. 


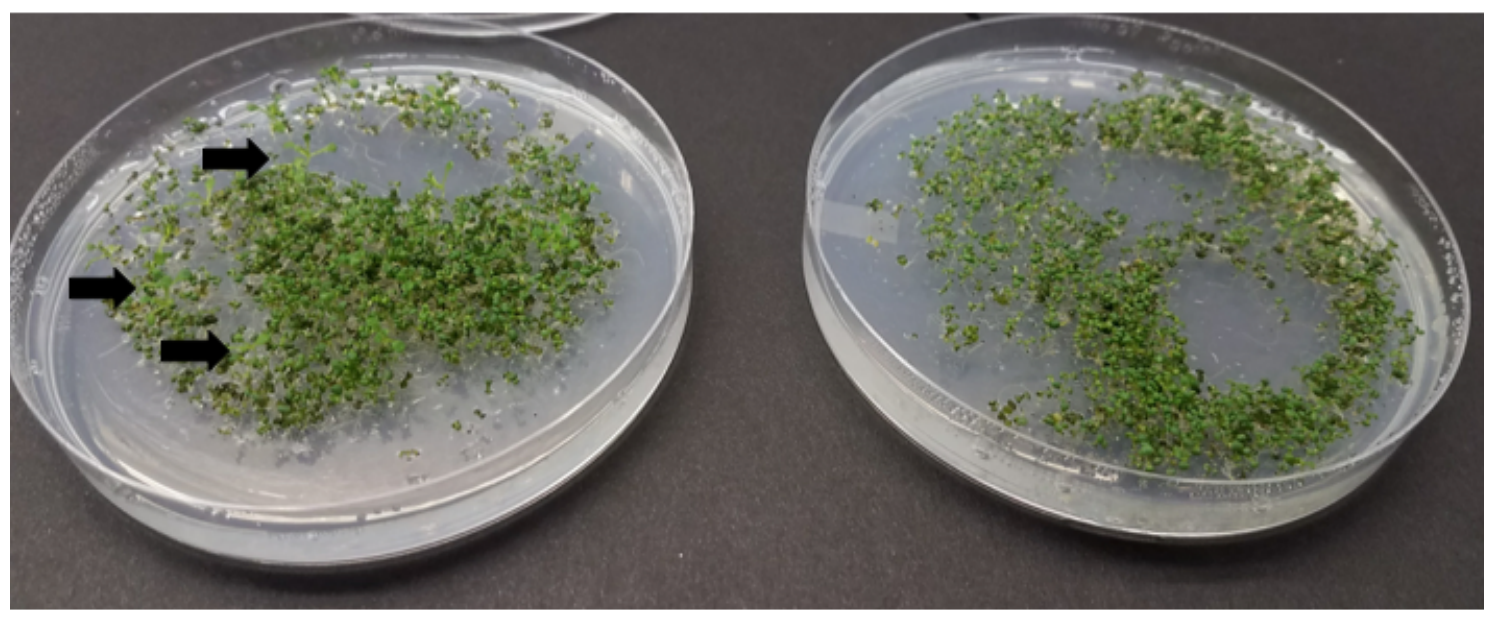

Figure 23 Example of Arabidopsis Dip Hygromycin Screen

Example of hygromycin screen results. Two plates are shown representing typical results. (Left) Positive control plate resulting from 35S Hygromycin dip. Positive plants, arrows, can be observed as growing taller than surrounding negatives. (Right) Experimental plate resulting from a plant containing an attachment site, Cre recombinase, and ФС 31 integrase, being dipped with pPZPBHygNosLox7166. No positive plants can be observed.

\section{Discussion}

\section{SSTI in Arabidopsis}

While the transformation process in Arabidopsis is not anywhere near as laborious as that in maize, it still relies on random integration into the genome, which can lead to many of the same detriments. By utilizing the same SSTI strategy in Arabidopsis researchers would be able to ensure that inserted transgenes are always located in the same genomic location. Comparative studies, for instance, would be able to ensure the same transcription level of different transgenes, taking away a level of complexity. 
The first attempts at SSTI utilized naturally occurring circular T-DNA complexes for integration. While previous work showed that circular DNA may be formed during Agrobacterium transformation and integration, this was not demonstrated in Arabidopsis and not with floral dips. As a result, it is not known if or how prevalent T-DNA circles are in the Arabidopsis floral dip procedure. Additionally, as the haploid ovule is the target of floral dip (Desfeux et al., 2000), it is not known if the SSTI mechanism would be active during this developmental stage. After experiments with a hygromycin construct were examined, no transgenic plants were found in the experimental seed screened. As the positive control plates showed transformed plants, the dip procedure was successful, suggesting that the SSTI procedure was not effective.

To investigate if the lack of T-DNA circles was the cause of the lack of transformants, plants containing Cre recombinase, ФС 31 integrase, and a target site were dipped with an integration construct that would be irreversibly circularized in the presence of Cre recombinase. Despite the forced circularization of the transgene, there were still no positive transformants present in the resulting progeny, even when 18000 plants were screened. It should be noted that the plants that were dipped could not be determined to be homozygous for each of the three constructs they contained. Even if each construct was heterozygous, however, around 40 plants of the expected 360 transformants should contain all three constructs if each is segregating independently. This result suggests that SSTI may not be a valid strategy when utilized with the floral dip procedure. The reason for this result is unknown, but 
may have to do with the unique target for transformation with floral dip in Arabidopsis.

\section{SSTI in Maize}

Transformation in maize is, in the current state, a complicated and time consuming procedure. Additionally, once the transformation procedure has been completed intense screening is required to ensure that transgenes are not interfering with endogenous genes and have adequate transcription. The result of random integration means that transgenic lines can have different transgene expression profiles, even if the same construct was introduced.

Through site-specific transgene insertion, many of the issues with maize transformation can be alleviated. The strategy, which targets incoming transgenes to a predetermined genomic location, allows expression to be determined before insertion and would not require transgenic lines be screened for insertion site locations. By predetermining insertion sites, current issues such as transgene mutagenesis can by bypassed.

In this study, the SSTI strategy utilizing $\Phi C 31$ integrase was demonstrated in maize embryos. Using the GUS reporter, a transgene was inserted into a previously inserted target site and expressed. The transgene reporter, which did not contain a promoter, was observed when introduced to embryos that already contained ФC31 integrase, and when cobombarded with ФC31 integrase sequence. The ability to introduce transgenes containing a target site, but not the ФC31 integrase protein, could allow for transgene integration through transient 
integrase expression. This would allow for transgenes to be recovered without requiring that $\Phi C 31$ integrase also be recovered.

The experiments utilizing GUS are not able to be confirmed molecularly as true integration events. It is not possible to use GUS as a selective agent for regeneration, and so transgenic plants cannot be recovered. However, by comparing the experimental embryos to those of the control, it is most likely that SSTI has occurred. The reporter construct, which consists of an attB site followed by the promoter-less GUS sequence, will only produce signal if inserted behind an active promoter. This is possible through random chance of insertion behind a promoter anywhere in the genome. Of the over 100 negative control embryos that were bombarded, one did show a small GUS signal (figure 20). This could be the result of insertion of the reporter construct behind an endogenous active promoter. Signal could also be the result of contamination of the bombardment system with positive control DNA or gold particles. In either case, signal could be detected on multiple embryos when integrase was present, and with transgenic integrase multiple signals on a single embryo. This suggests that presence of integrase was the cause of an increase of signal, indicating that SSTI has indeed occurred.

While the GUS experiments allowed a relatively quick demonstration that SSTI may be possible in maize, a transgene must be introduced that contains a selection gene for regeneration to confirm SSTI molecularly. With the current transgenic lines, this is problematic as the ФC31 integrase line used, INT7, contains a permanent bialaphos selection gene. As a result, bialaphos selection, 
the only selection agent routinely used in academic labs for maize regeneration, cannot be used for SSTI with the current line. To use the current SSTI strategy with the INT7 line, a different method of selection must be used. In a recent review of industry patents (chapter 1), it was found that many industry labs use glyphosate selection during maize transformation. Utilizing glyphosate for selection would allow for the current INT7 integrase line to be used with the newly introduced transgene. The plasmid pB34Glyphosate (figure 23) was designed and synthesized to be introduced into GLP target sites for glyphosate selection.

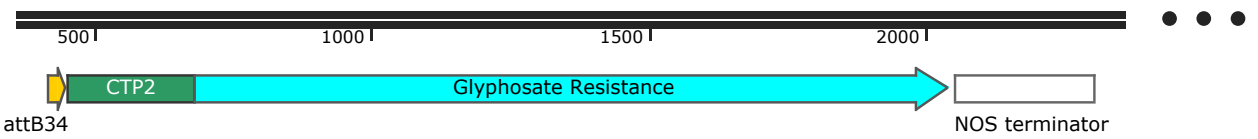

pB34Glyphosate
4512 bp

Figure 24 Insertion Construct for Glyphosate Selection

Insertion construct designed for SSTI with glyphosate selection. The construct consists of an attB site followed by a glyphosate resistance gene targeted to the chloroplast.

While it is not possible to get a true estimate of SSTI efficiency with the GUS experiments, some observations can be made. First, it should be noted that the Actin-Gus positive control cannot be used for a comparison of transformation efficiency. There is no way of determining how much of the GUS signal in the positive control is a result of integration and how much is a result of transient expression. The SSTI reporter construct, however, can only be observed in 
genomic insertion has occurred. By comparing the different insertion strategies, however, it can be observed that the efficiency was much higher when integrase was already expressing in the embryo. This is not surprising because an embryo already expressing the integrase protein will have it expressed in every cell. Consequently, each time the incoming transgene enters the nucleus it has a chance of being recognized and integrated. In the case of cobombardment, both the recombination protein and transgene must enter the nucleus together, come into contact, and then also come into contact with the target site. The increase in complexity will result in a lower efficiency.

Another observation can be made from the GUS experiments by comparing GUS signals on a single embryo. In the case of cobombardment, there were never any cases observed that resulted in multiple insertions on the same embryo. In the case of the already expressing integrase experiments, however, multiple insertions occurred on most embryos examined. This is another observation that suggests that SSTI is more efficient when integrase is already expressed. In the case of normal transformation strategies multiple insertions would not be desirable; however in SSTI, this is not the case. This is due to the fact that in normal transformation multiple insertions on a single embryo could result in a chimeric plant if the resulting callus is not sufficiently separated. In SSTI, however, each insertion would be into the same genomic location, meaning that combining insertions during regeneration would result in a single event. In a practical sense, the ability to combine transformation events 
during regeneration would lead to an easier regeneration scheme as less care would need to be taken while maintaining callus lines.

When comparing efficiency of SSTI to overall transformation efficiency, there are additional points to consider. The embryos used for these experiments were not allowed to develop into full plants, so a direct comparison cannot be made. Additionally, there is no way of knowing how often traditional transformation methods result in integrations on a single embryo. Furthermore, a developing embryo does not grow uniformly. Every cell of an embryo is not capable of developing into callus, and often large sectors will die during development. As such, it is possible that many of the observed insertion sites may not have the ability of developing into full plants. However, this is less likely the case when using embryos already containing the integrase sequence, as there are many insertions on each embryo. Even if a few insertions were lost during development on the embryo shown in figure 19, there are enough additional insertions that it is likely that one would make it through the procedure. It is of note that in the case of normal transformation, efficiency is measured by comparing the total number of transformed lines to the number of embryos originally treated. This means that for a typical transformation only $5-10 \%$ of the original embryos have insertions that are recoverable. While it is difficult to make a direct comparison with the GUS experiments, it is of note that many embryos contain multiple insertions. The treated embryo in figure 19 for instance has at least 6 insertions visible through microscopy. The embryos used for this study are heterozygous for both target site and integrase, so it is possible that when 
homozygous for both transgenes the number of SSTI events could increase further. This is of note as it increases the chances of each embryo developing callus sectors that contain new transgenes. If every embryo in a sample contains both a target and integrase protein, it is possible that new transgenes could be introduced to each, drastically increasing transformation efficiency.

Transformation efficiency would be further improved through SSTI as each insertion event would be known to be located in an area of expression that is sufficient for selection. This is because the same target locus was used previously for selection when the target site was originally inserted. As such, each inserted transgene should have expression high enough for recovery. There are additional factors that may lead to a lack of transgene recovery however. First, it is important to consider that developing callus is three dimensional, and as a result, often relies on tissue below it to obtain nutrients. In the case of selection, this means that callus may be killed that contains resistance protein sufficient for resistance as a result of callus below it dying. While callus is routinely divided and spread on growth media, it is difficult to prevent sectors from dying during the procedure. The ability to introduce multiple insertions in one developing callus line would increase the chances of an event making it through the process.

The site-specific integration strategy utilized here should allow for efficient integration of transgenes; however, it would only allow for a single transgene to be introduced. This would require multiple transgenes to be crossed together and maintained through crossing schemes. In an ideal situation, transgenes could be 
linked to prevent segregation during crossing. Additionally, while attempts are being made to utilize alternative selection genes, current academic strategies utilize bialaphos exclusively. As a result, for recurrent integration it may be best to design a system that can use bialaphos for each transformation event. To achieve this, the incoming transgene could be designed to incorporate an additional recombination site that could be targeted in future transformation events while also introducing a removable bialaphos resistance gene (figure 24).

In this strategy, the chosen selection gene could be reused each time a new transgene is introduced. Following regeneration, the transgenic line is crossed to a Cre recombinase containing line to remove the selection gene. After this cross is completed a new recombination site can be targeted that will be located directly behind the originally introduced promoter. The site can be targeted for SSTI by another integration construct that reintroduces selection and also carries additional recombination sites. Similar to the first strategy the regenerated plant can be crossed to a Cre recombinase containing line to remove selection again. After this reduction the original integration construct can be re-used with a new GOI inserted. This strategy can then be continued for as many transgenes are desired at a given locus. 
A.

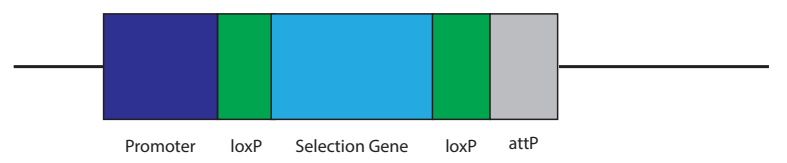

$+\quad$ cre

B.

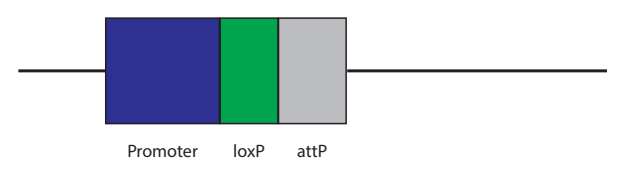

$+$

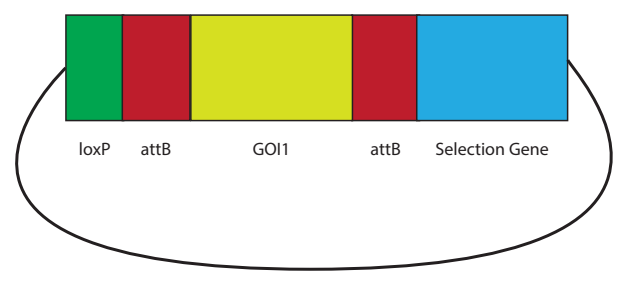

+ Integrase

C.
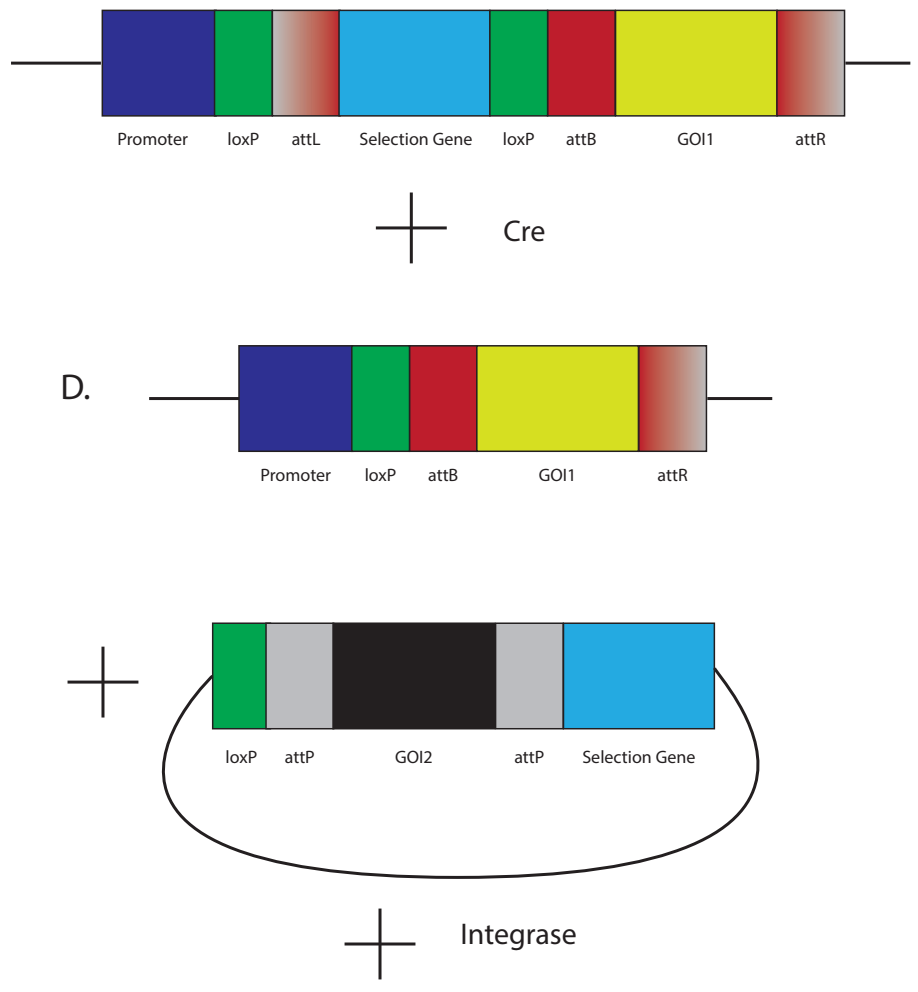

E.

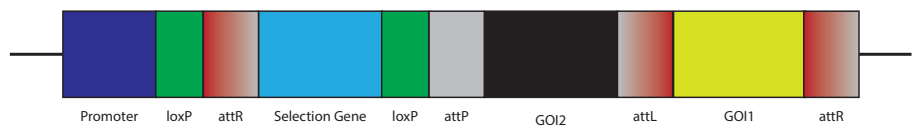


Figure 25 Recurrent SSTI Scheme for Maize

Strategy for recurrent integration into a single genomic locus in maize using ФC31 integrase. (A) A target site is introduced that contains a promoter driving a removable selection gene followed by an attP site. (B) The target site line is crossed to a line containing Cre recombinase to remove selection. (C) An integration construct is introduced either into a line that already contains integrase, or cobombarded with the integrase sequence. The integration construct contains a loxP site, a gene of interest (GOI) that is flanked by two attB sites, and a selection gene. Upon entering the nucleus $\Phi C 31$ integrase can recognize either of the attB sites being introduced. However, only recombination with the second site will integrate the selection gene behind the target site promoter and be selected for. (D) After integration the selection gene will be driven by the target site promoter and be flanked by loxP sites for removal by Cre recombinase. (E) A second integration construct containing a second GOI can then be introduced to the newly reduced target site with attP sites. As in the first integration construct, only recombination with the second attP site will introduce an active selection gene. Following integration the selection gene is again flanked by loxP sites which would allow for subsequent removal by Cre recombinase. Following this reduction, the first integration construct containing a third GOI could be introduced. This scheme could continue ad infinitum.

While this strategy for recurrent SSTI would be successful in inserting multiple transgenes into a single locus, it may not be the most efficient method for doing so. First, the system relies on recombination between the correct recombination site of the incoming construct. As the strategy requires an additional recombination site be inserted for future insertions, the incoming transgene will contain two recombination sites. The selection gene will only be active if the correct site is located; however, there is no way to control which site is recognized. As a result, there is a $50 \%$ reduction in efficiency due to 
unselectable recombination. Additionally, the entire strategy requires outcrossing to Cre recombinase to remove selection, then removing Cre recombinase before the next round of transformation can be attempted. The T0 transgenic line can be crossed immediately to Cre recombinase after regeneration; however, the system will still require a round of outcrossing to remove the Cre protein from the system before the next round of transformation, a process that will take a minimum of 3 months. Additionally, as only a single recombination site is utilized, the entire plasmid backbone is integrated along with the GOI. While the maintenance sequences will be removed when the selection gene is removed, it is still not desirable for transgenic plant production to have them included.

The recurrent SSTI strategy can be improved by first utilizing alternate attachment site sequences. Previous work has shown that the recombination reaction for $\Phi C 31$ integrase requires exact specificity of a 2 bp overlap sequence and when altered, the recombination sites will only recombine with similarly mutated sites (Colloms et al., 2013). By utilizing mutated sites, only a single integration site will be recognized on the incoming transgene construct, eliminating the inefficiency of the previous strategy. Additionally, as previous work has shown that other selection strategies can be utilized in maize (chapter 2, Howe et al., 2002), a system can be designed that does not require the reduction of a target site before a subsequent round of transformation. By combining these two modifications, a more efficient recurrent SSTI strategy could be employed (figure 26). 
In this strategy, two recombination events are required for each integration, causing a cassette exchange of desired sequences. The result is only the desired sequences being integrated into the target site as opposed to the entire plasmid. By utilizing mutated overlap sequences for recombination sites, there is no risk of the wrong sites being recombined during transformation. This also allows for multiple attachment sites to be used throughout the genome while also maintaining a high efficiency. The system relies on two main integration constructs, each with a different selection gene. By switching back and forth between different types of resistance, there is no-longer a requirement of crossing to a different recombination protein to remove the original selection gene. The system does not have an inherent strategy to only remove the selection gene; however, this could be accomplished by also flanking the selection gene with loxP sites. 

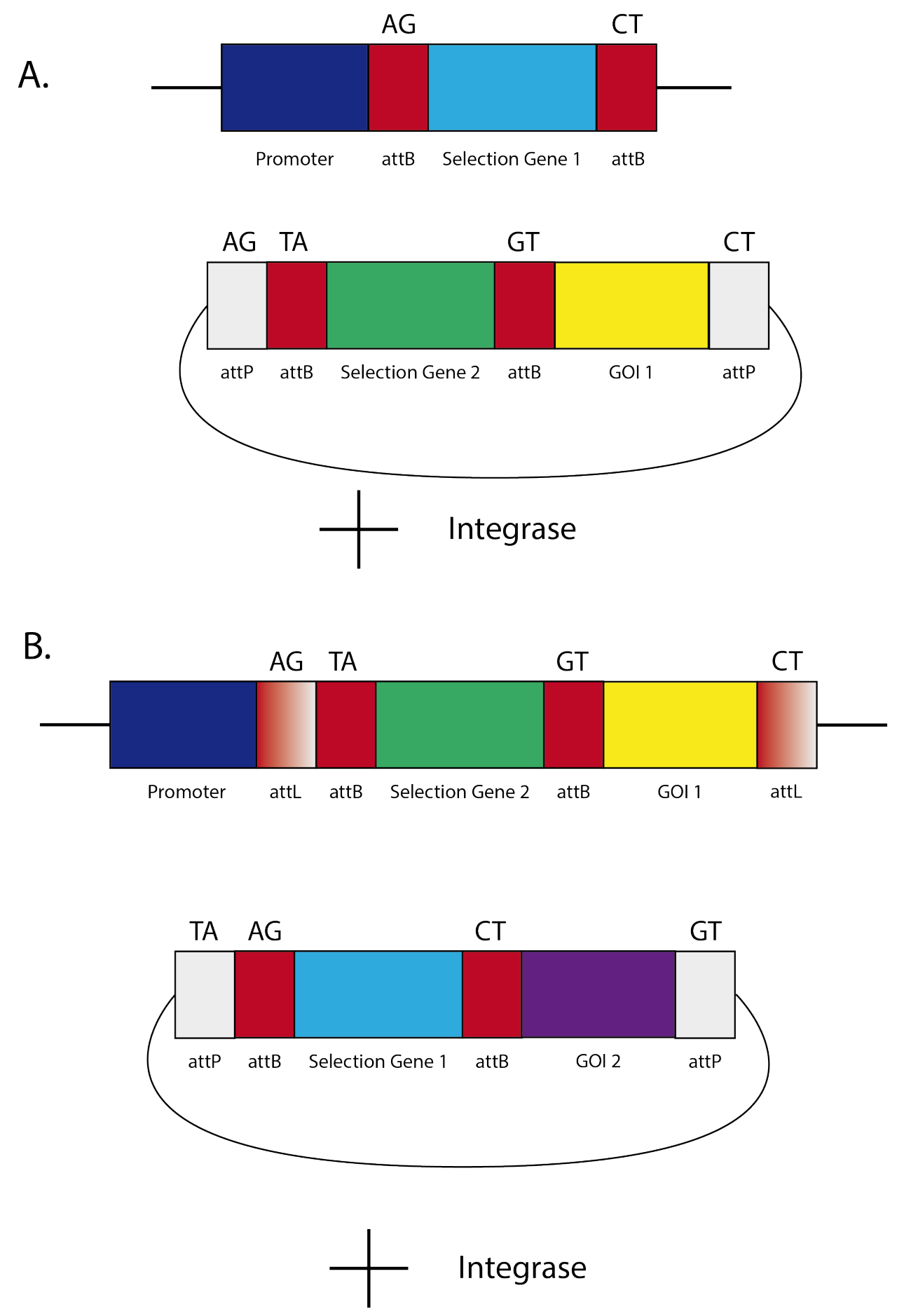

C.

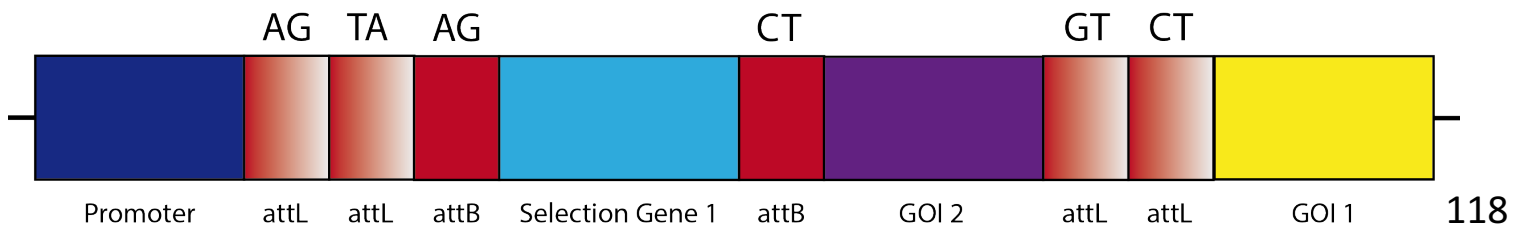


Figure 26 Alternate Recurrent SSTI Strategy

Alternate strategy for repeated transgene addition through SSTI. (A) A target is first placed into the genome that contains a promoter driving a selection gene. The selection gene is flanked by two attB sites, each with different 2 bp overlap sequences, indicated by labels above attachment sites. (B) An integration construct is introduced into either an integrase containing line or by combombarding integrase sequence. This construct contains attP sites with the same $2 \mathrm{bp}$ overlap sequences as the already introduced attB sites flanking the insert cassette. The insert cassette consists of a different selection gene flanked by new attB sites with different 2 bp overlap sequences, and followed by a GOI. Upon recombination, the matching attB and attP sites will recombine into attL sites, inserting only the intervening cassette without the plasmid backbone. This new event can be recovered by selecting for the new resistance gene. (C) Another integration event can be carried out immediately by utilizing a new integration construct. This integration construct is similar to the first, it contains attP sites that match the target site, a new selection gene, attB sites for future integrations, and another GOI. The next attB sites introduced can have the same overlap sequences as the first, allowing for the first integration construct to be utilized again. Following recombination, a new selection gene will now be present in the target site for regeneration as well as new attB sites for future targeting.

No matter what strategy is used for SSTI in maize, a target site must first be introduced into the genome. This is accomplished by random integration by either particle bombardment or Agrobacterium tumefaciens standard techniques. To make the system robust for many different applications it would be advantageous to create a library of insertion sites throughout the genome. This would allow researchers to choose from a variety of insertion locations with differing expression patterns. Due to the small size of the original target site, it 
may be possible to introduce the target sites through an endonuclease. The use of Cas9 for SSTI has been shown to work in maize (Svitashev et al., 2015), though was inefficient. There has also been limited success in other organisms by utilizing a geminivirus that creates many copies of a chosen DNA fragment (Baltes et al., 2014). The amount of sequence that can be maintained in a geminivirus is limited; however, the small size of recombination sites may allow for their replication on a geminivirus molecule. By combining a target site with guide-RNA molecules it may be possible to place target sites virtually anywhere in the genome. By choosing where the target site is placed, a locus can be chosen that is not linked to undesirable traits and would not interfere with native gene sequences.

Another application for SSTI in maize is the modification of minichromosomes. Minichromosomes, which are small chromosomes that exists outside of the normal chromosome set in an organism, allow researchers to place transgenes into an organism without the risk of interfering with native genes. Additionally, as they are separate from the normal chromosome set there is no risk of linkage so they can be transferred to different lines easily. Minichromosomes are produced through telomere truncation; a transgene is cointroduced with telomere sequences, which are recognized by native machinery during transformation and assembled into a new chromosome end (Yu et al., 2006). As the process will result in the loss of chromosomal material distal to the insertion site, in maize the process is usually carried out on lines that contain the supernumerary B chromosome, which would not have a phenotypic effect with 
the loss of genetic material. The process can be carried out with either particle bombardment or Agrobacterium transformation techniques, but ultimately still relies on random integration. As a result, minichromosome production is inefficient as it relies on an insertion on the B chromosome and then recognition of the telomere sequences by the native machinery. Additionally, as there is currently no way to target minichromosomes once they have been produced, additional transgenes must be inserted onto new minichromosomes. Either type of recurrent SSTI strategy (figures 25 or 26 ) could be adapted to work with minichromosome technology by inserting telomere sequences after the target site. The target site is then introduced to a line containing B chromosomes and screened for a minichromosome after regeneration. A minichromosome that contains a target site can then be utilized for future transgene insertions outside of the maize genome.

Transformation in maize is currently a long and expensive process. This is made worse by the fact that transgenes may be unusable afterwards due to endogenous gene interference or linkage to unwanted alleles. Through sitespecific transgene insertion, many of these problems can be alleviated. The work shown here lays the groundwork for the SSTI in maize, and should be applicable to other systems as well.

\section{References}

Albert, H., Dale, E., Lee, E., and Ow, D. (1995). Site-specific integration of DNA into wild-type and mutant lox sites placed in the plant genome. The Plant 
Journal : For Cell and Molecular Biology 7, 649-659.

Baltes, N.J., Gil-Humanes, J., Cermak, T., Atkins, P. a, and Voytas, D.F. (2014). DNA replicons for plant genome engineering. The Plant Cell 26, 151-163. Brunaud, V., Balzergue, S., Dubreucq, B., Aubourg, S., Samson, F., Chauvin, S., Bechtold, N., Cruaud, C., DeRose, R., Pelletier, G., et al. (2002). T-DNA intergration into the Arabidopsis genome depends on sequences of preinsertion sites. EMBO Reports 3, 1152-1157.

Christensen, a H., and Quail, P.H. (1996). Ubiquitin promoter-based vectors for high-level expression of selectable and/or screenable marker genes in monocotyledonous plants. Transgenic Research 5, 213-218.

Christou, P. (1991). Genetic transformation of crop plants using microprojectile bombardment. The Plant Journal 2, 275-281.

Clough, S.J., and Bent, A.F. (1998). Floral dip: A simplified method for Agrobacterium-mediated transformation of Arabidopsis thaliana. Plant Journal $16,735-743$.

Colloms, S.D., Merrick, C. a, Olorunniji, F.J., Stark, W.M., Smith, M.C.M., Osbourn, A., Keasling, J.D., and Rosser, S.J. (2013). Rapid metabolic pathway assembly and modification using serine integrase site-specific recombination. Nucleic Acids Research 1-10.

Dale, E., and Ow, D. (1991). Gene transfer with subsequent removal of the selection gene from the host genome. Proceedings of the National Academy of Sciences of the United States of America 88, 10558-10562.

Desfeux, C., Clough, S.J., and Bent, A.F. (2000). Female reproductive tissues 
are the primary target of Agrobacterium-mediated transformation by the Arabidopsis floral-dip method. Plant Physiology 123, 895-904.

Graham, N.D., Cody, J.P., Swyers, N.C., McCaw, M.E., Zhao, C., and Birchler, J.A. (2015). Engineered Minichromosomes in Plants. In International Review of Cell and Molecular Biology, (Elsevier Ltd), pp. 63-119.

Groth, A.C., Olivares, E.C., Thyagarajan, B., and Calos, M.P. (2000). A phage integrase directs efficient site-specific integration in human cells. Proceedings of the National Academy of Sciences 97, 5995-6000.

Hou, L., Yau, Y.Y., Wei, J., Han, Z., Dong, Z., and Ow, D.W. (2014). An OpenSource System for in Planta Gene Stacking by Bxb1 and Cre Recombinases. Molecular Plant 7, 1756-1765.

Howe, A.R., Gasser, C.S., Brown, S.M., Padgette, S.R., Hart, J., Parker, G.B., Fromm, M.E., and Armstrong, C.L. (2002). Glyphosate as a selective agent for the production of fertile transgenic maize (Zea mays L.) plants. Molecular Breeding 10, 153-164.

Keravala, A., Groth, A.C., Jarrahian, S., Thyagarajan, B., Hoyt, J.J., Kirby, P.J., and Calos, M.P. (2006). A diversity of serine phage integrases mediate site-specific recombination in mammalian cells. Molecular Genetics and Genomics 276, 135-146.

Kim, S.-I., and Gelvin, S.B. (2007). Genome-wide analysis of Agrobacterium TDNA integration sites in the Arabidopsis genome generated under nonselective conditions. The Plant Journal 51, 779-791.

Koncz, C., Martini, N., Mayerhofer, R., Koncz-Kalman, Z., Korber, H., Redei, 
G.P., and Schell, J. (1989). High-frequency T-DNA-mediated gene tagging in plants. Proceedings of the National Academy of Sciences 86, 84678471.

McCaw, M., Swyers, N., Graham, N., Cody, J., Zhao, C., and Birchler, J. (2016a). Preparation of Chromosomes from Zea mays. Current Protocols in Plant Biology 1, 501-509.

McCaw, M., Graham, N., Cody, J., Swyers, N., Zhao, C., and Birchler, J. (2016b). Fluorescence In Situ Hybridization to Maize (Zea mays) Chromosomes. Current Protocols in Plant Biology 1, 530-545.

Medberry, S.L., Dale, E., Qin, M., and Ow, D.W. (1995). Intra-chromosomal rearrangements generated by Cre-lox site-specific recombination. Nucleic Acids Research 23, 485-490.

Oard, J.H., Paige, D.F., Simmonds, J.A., and Gradziel, T.M. (1990). Transient Gene Expression in Maize, Rice, and Wheat Cells Using an Airgun Apparatus. Plant Physiology 92, 334-339.

Ow, D.W. (2011). Recombinase-mediated Gene Stacking as a Transformation Operating System. Journal of Integrative Plant Biology 53, 512-519.

Sallaud, C., Gay, C., Larmande, P., Bès, M., Piffanelli, P., Piégu, B., Droc, G., Regad, F., Bourgeois, E., Meynard, D., et al. (2004). High throughput TDNA insertion mutagenesis in rice: A first step towards in silico reverse genetics. Plant Journal 39, 450-464.

Singer, K., Shiboleth, Y.M., Li, J., and Tzfira, T. (2012). Formation of Complex Extrachromosomal T-DNA Structures in Agrobacterium tumefaciens- 
Infected Plants. Plant Physiology 160, 511-522.

Songstad, D.D., Armstrong, C.L., Petersen, W.L., Hairston, B., and Hinchee, M. a W. (1996). Production of Transgenic Maize Plants and Progeny by Bombardment of Hi-II Immature Embryos. In Vitro Cellular \& Developmental Biology - Plant 32, 179-183.

Srivastava, V., and Ow, D.W. (2004). Marker-free site-specific gene integration in plants. Trends in Biotechnology 22, 627-629.

Svitashev, S., Young, J.K., Schwartz, C., Gao, H., Falco, S.C., and Cigan, A.M. (2015). Targeted Mutagenesis, Precise Gene Editing, and Site-Specific Gene Insertion in Maize Using Cas9 and Guide RNA. Plant Physiology $169,931-945$.

Thompson, C., Movva, N., Tizard, R., Crameri, R., Davies, J., Lauwereys, M., and Botterman, J. (1987). Characterization of the herbicide-resistance gene bar from Streptomyces hygroscopicus. The EMBO Journal 6, 25192523.

Vega, J.M., Yu, W., Kennon, A.R., Chen, X., and Zhang, Z.J. (2008). Improvement of Agrobacterium-mediated transformation in $\mathrm{Hi}$-II maize (Zea mays) using standard binary vectors. Plant Cell Reports 27, 297305.

Vergunst, A.C., Jansen, L.E.T., and Hooykaas, P.J.J. (1998). Site-specific integration of Agrobacterium T-DNA in Arabidopsis thaliana mediated by Cre recombinase. Nucleic Acids Research 26, 2729-2734.

Yu, W., Lamb, J.C., Han, F., and Birchler, J. a (2006). Telomere-mediated 
chromosomal truncation in maize. Proceedings of the National Academy of Sciences 103, 17331-17336.

Zhang, W., Subbarao, S., Addae, P., Shen, A., Armstrong, C., Peschke, V., and Gilbertson, L. (2003). Cre/lox-mediated marker gene excision in transgenic maize (Zea mays L.) plants. Theoretical and Applied Genetics 107, 1157-1168. 


\section{Chapter 4 Production of Minichromosome Vectors via the Introduction of Telomere Sequences}

As published in:

Graham, N., Swyers, N., Cody, J., McCaw M., Zhao, C., and Birchler J. (2016). Production of Engineered Minichromosome Vectors via the Introduction of Telomere Sequences. Chromosome and Genomic Engineering in Plants: Methods and Protocols, Methods in Molecular Biology. 1469. 1-13.

Nathaniel Graham, Nathan Swyers, Jon Cody, Morgan McCaw, Changzeng Zhao, and James A. Birchler

\section{Summary}

Artificial minichromosomes are non-integrating vectors capable of stably maintaining transgenes outside of the main chromosome set. The production of minichromosomes relies on telomere-mediated chromosomal truncation, which involves introducing transgenes and telomere sequences concurrently to the cell to truncate an endogenous chromosomal target. Two methods can be utilized, either the telomere sequences can be incorporated into a binary vector for transformation with Agrobacterium tumefaciens, or the telomere sequences can be co-introduced with transgenes during particle bombardment. In this protocol, the methods required to isolate and introduce telomere sequences are 
presented. Following the methods presented, standard transformation procedures can be followed to produce minichromosome containing plants.

Key Words: Minichromosome, chromosomal truncation, artificial chromosome, telomere, in-gel ligation, plant transformation, Agrobacterium tumefaciens transformation, biolistic bombardment

\section{Introduction}

Artificial minichromosomes are any non-integrating vectors with large DNA carrying capacities that can be stably maintained in sequential generations (Gaeta et al., 2012). Production of minichromosomes is a result of telomere truncation, where telomere sequences are introduced into a chromosome during transformation resulting in the loss of genetic material distal to the insertion point. Telomere mediated truncation is accomplished through transformation of a plant with a plasmid containing a telomere repeat after the selectable marker and other desired genetic cargo, or by particle bombardment with a plasmid and separate telomere repeat. It is presumed that the non-homologous end joining pathway for double strand break repair attaches the plasmid to a double-stranded break in an endogenous chromosome. The presence of the telomere sequence then recruits telomerase, which adds telomere repeats to the end of the plasmid, creating a functional end of the chromosome. B chromosomes in maize and other plants are good targets for creating minichromosomes because they contain no genes essential to the survival of the plant. If an A chromosome is truncated, the event will generally result in the loss of genes, which are essential to the survival of the 
plant, making recovery of truncation events less likely. Truncating a B chromosome will have little to no effect on the survival of the plant. The use of a B chromosome has the added benefit of having no linkage between the transgene and endogenous genes in the transformed line. Transgenes on an A chromosome may be linked to alleles from the transformable line, which are undesirable in a high yielding commercial line. A suite of transgenes can be carried on a single minichromosome and introgressed into new lines as a single unit, reducing the complexity of breeding programs to stack multiple different transgenes in a single plant. The complexity and duration of introgressing multiple transgenes into a line may possibly be further reduced by transferring minichromosomes through haploid induction and doubling the ploidy of a resultant haploid containing a minichromosome would create a completely homozygous line with minichromosomes (Gaeta et al., 2012).

Minichromosomes exist in association with the normal chromosome set and are subject to modification via site-specific recombination technology. Modifications add or remove gene fragments in a targeted manner, enabling continuous concatenation of sequences while recycling a single selection marker. As minichromosome technology develops, this strategy could allow researchers to stack multiple genes or whole biosynthetic pathways on a single location within the genome. This would circumvent limitations associated with current popular genetic engineering methods, such as disruption of endogenous gene function, transgene silencing, linkage drag, and inefficient recovery of multiple transgenes (Yu et al., 2007a). 
This system relies on the development of a minichromosome platform, which is used as a target for subsequent modification events. In plants, platforms are produced through telomere-mediated truncation of pre-existing genetic material via Agrobacterium tumefaciens transformation or particle bombardment. Each method requires the utilization of a telomere array; however, the mode of delivery and materials used in these processes are slightly different. Agrobacterium transformation requires an advanced cloning strategy to position a telomere fragment near the right border of a T-DNA vector, while particle bombardment simply requires an isolation of the telomere sequence. Preparation of telomere for both cloning or bombardment can be carried out in two different ways, through gel extraction or telomere repeat concatenation via PCR.

Due to the difficulty in manipulating the repetitive sequences of the telomere, it must be moved to into a transformation vector via in-gel ligation. After positive clones have been identified they must be screened and sequenced to ensure that the insert is intact and in the correct orientation. Following sequencing of positive colonies, those in the correct orientation should be screened for insert size. The minimum size required for telomere truncation has not yet been determined, but it is thought that the greatest chance of success will come from the use of the largest telomere sequence possible. Interestingly, telomere often forms a secondary structure within agarose gels making it difficult to get an accurate size estimate. Performing a Southern hybridization (Southern, 1975) on these agarose gels will show evidence of the full size of the telomere repeat that is present in a clone. 
For particle bombardment, telomere DNA conglomerates can be produced using polymerase chain reaction). Differing lengths of telomere DNA are created by the annealing of specific primers to each other resulting in the creation of telomere repeats of varying sizes. The resulting fragments of telomere can be visualized by gel electrophoresis and particular sizes of telomere can be obtained by DNA gel extraction from an agarose gel. The obtained telomere DNA can then be used in particle bombardment with a construct of interest to create a minichromosome. The protocol described in the following section has been adapted from a protocol that labels telomere to make fluorescent probes (ljdo et al., 1991).

\section{Protocol 1: Ligation of Telomere Sequences within}

\section{Agrobacterium transformation vectors}

\section{Materials}

\subsection{Materials for in-gel ligation into Agrobacterium competent vector}

1. Agrobacterium tumefaciens competent transformation vector (see Note 1)

2. Plasmid pWY82 (see Note 2)

3. Oligonucleotide (TTTAGGG) ${ }_{10}$, can be synthesized in either the 5' or 3' direction

4. Luria broth

5. Spectinomycin 
6. 2xYT Medium

7. QIAprep Spin Miniprep Kit (Qiagen)

8. Restriction Enzymes compatible with pWY82 and target plasmid

9. Agarose

10. DNA Gel Loading Dye (6X)

11. GeneRuler 1 kb DNA Ladder (Life Technologies)

12. Ethidium Bromide

13. Low Melting Point Agarose

14. Antarctic phosphatase

15. T4 DNA Ligase and Ligase Buffer

16. ElectroMax Stbl4 Cells (Life Technologies)

17.S.O.C Media (Super Optimal Broth with Catabolic Repressor)

18. Agar

19. Petri Dishes

2.2 Equipment for in-gel ligation into Agrobacterium competent vector

1. $30^{\circ} \mathrm{C}$ Incubator

2. $30^{\circ} \mathrm{C}$ Shaker

3. $250 \mathrm{~mL}$ baffled culture flasks

4. Vacuum Concentrator

5. Nanodrop Spectrophotometer

6. Gel electrophoresis system 
7. Ultraviolet Transilluminator

\subsection{Recipes for in-gel ligation into Agrobacterium competent vector}

1. Luria Broth: For $500 \mathrm{~mL}$ dissolve $12.5 \mathrm{~g}$ of LB media in $400 \mathrm{ml}$ water. Bring to $500 \mathrm{~mL}$ and autoclave for 20 minutes.

2. Luria Broth Plates: For $500 \mathrm{~mL}$ dissolve $12.5 \mathrm{~g}$ of $\mathrm{LB}$ media and $6 \mathrm{~g}$ of agar is $400 \mathrm{~mL}$ water. Bring to $500 \mathrm{~mL}$ and autoclave for 20 minutes. Place in $50^{\circ} \mathrm{C}$ waterbath until completely cooled then add appropriate antibiotics. Gently mix and pour thin layer into petri dishes. Store at $4^{\circ} \mathrm{C}$ for up to 1 month.

3. $2 x Y T$ Broth: For $500 \mathrm{~mL}$ of culture dissolve $15.5 \mathrm{~g}$ of $2 x \mathrm{YT}$ in $400 \mathrm{~mL}$ water. Bring final volume to $500 \mathrm{~mL}$ and autoclave for 20 minutes.

4. TAE: To prepare $1 \mathrm{~L}$ of $50 \mathrm{X}$ TAE add $242 \mathrm{~g}$ trizma base, $14.6 \mathrm{~g}$ EDTA, and $57.1 \mathrm{~mL}$ of acetic acid to $500 \mathrm{~mL}$ of water and dissolve. Bring total volume to $1 \mathrm{~L}$ with water.

\subsection{Materials for preparation of telomere for particle bombardment}

1. Telomere Primers (see Note 3):

a. Forward Primer- 5' (TTTAGGG) 10 3'

b. Reverse Primer- 5' (CCCTAAA) 10 3'

2. LongAmp® Taq DNA Polymerase (New England BioLabs)

3. DNA Gel Loading Dye (6X)

4. GeneRuler $1 \mathrm{~kb}$ DNA Ladder (Life Technologies)

5. Ethidium Bromide 
6. Wizard® SV Gel and PCR Clean-Up System (Promega)

7. Nuclease-Free Water

\subsection{Equipment for preparation of telomere for particle bombardment}

1. Gel electrophoresis system

2. Ultraviolet Transilluminator

\section{Methods}

\subsection{Insertion of Telomere through In-Gel Ligation}

1. Streak pWY82 onto LB plates containing $100 \mathrm{mg} / \mathrm{ml}$ spectinomycin.

2. Place plates into $30^{\circ} \mathrm{C}$ incubator for 48 hours (see Note 4).

3. Begin a starter culture by picking a single colony into $3 \mathrm{~mL}$ of $2 x Y T$ liquid media containing $100 \mathrm{mg} / \mathrm{ml}$ spectinomycin and shaking for 48 hours at $30^{\circ} \mathrm{C}$.

4. Add $500 \mu \mathrm{l}$ of starter culture to $125 \mathrm{~mL}$ of $2 \mathrm{xYT}$ in a baffled culture flask and shake at $250 \mathrm{rpm}$ at $30^{\circ} \mathrm{C}$ until the culture reaches an OD600 2 ( 48 hours).

5. Extract culture $4 \mathrm{~mL}$ at a time with the QIAprep Spin Miniprep kit (Qiagen) eluting with $50 \mu \mathrm{l}$ of $50^{\circ} \mathrm{C}$ nuclease free water.

6. Combine each miniprep into one $1.7 \mathrm{~mL}$ tube and reduce the volume in a vacuum concentrator until the concentration is $\sim 1 \mu \mathrm{g} / \mu \mathrm{l}$ when measured with a Nanodrop spectrophotometer. 
7. Individually test each restriction enzyme to be used by digesting $1 \mu \mathrm{g}$ of plasmid following manufacturers instructions.

8. Add $6 x$ loading dye to each digest after digestion is complete.

9. Load each digest into a $1 \%(w / v)$ TAE agarose gel flanked by GeneRuler $1 \mathrm{~kb}$ DNA ladder.

10. Run gel until loading dye approaches bottom of gel.

11. Stain gel with $0.5 \mu \mathrm{g} / \mathrm{mL}$ ethidium bromide for 30 minutes.

12. Visualize gel under UV light to check integrity of plasmid and efficiency of restriction enzymes (see Note 5). 


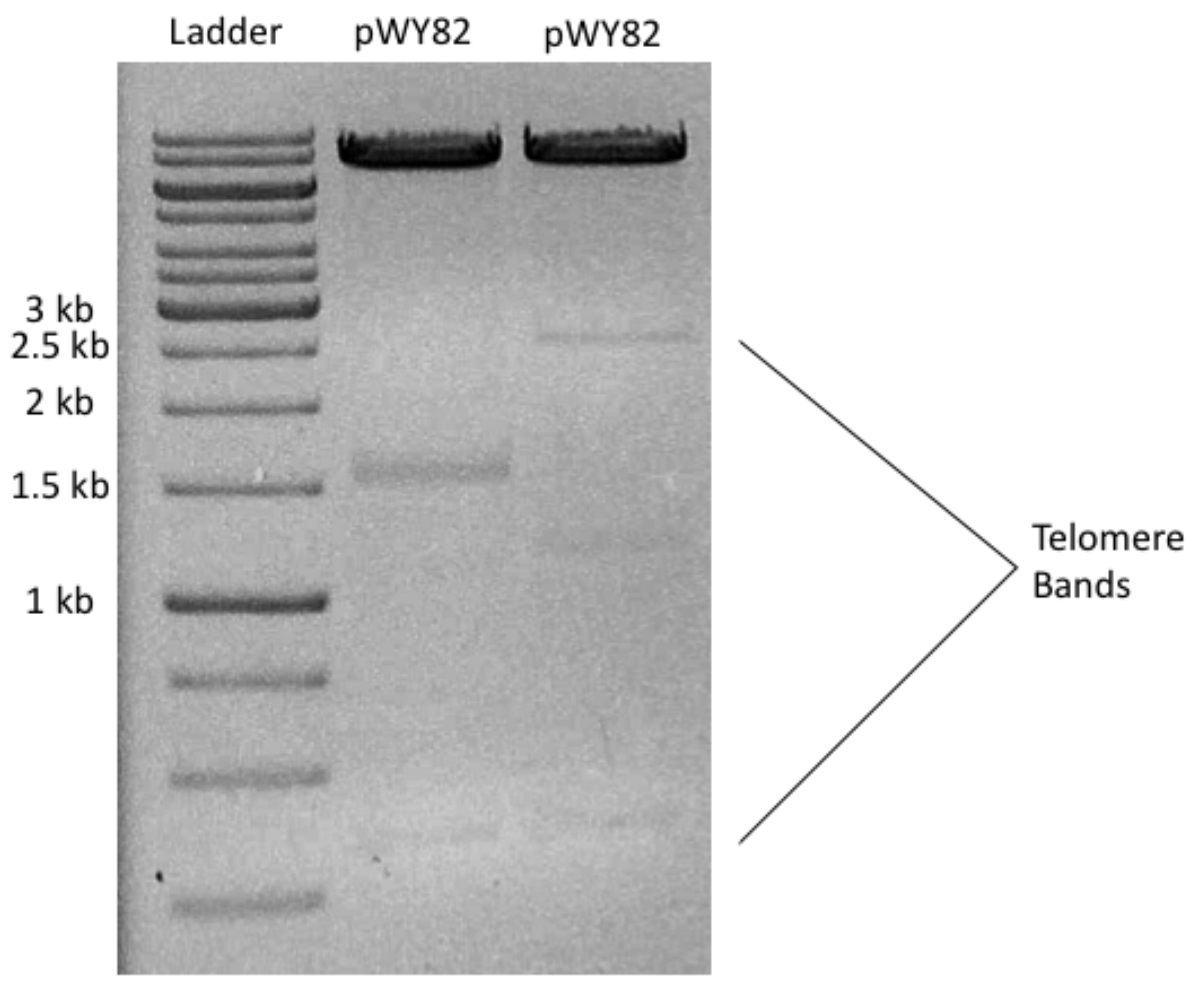

Figure 27 Example Digest of pWY82.

Plasmid pWY82 in both lanes was cut with EcoRv and HindIII though different sized bands can be seen throughout the gel.

13. Digest $10 \mu \mathrm{g}$ of pWY82 and $5 \mu \mathrm{g}$ of the target plasmid with 10 units of each restriction enzyme according to the manufacturer's instructions in a $50 \mu$ total volume.

14. Prepare a $1 \%(w / v)$ TAE low melting point agarose gel and allow to solidify for 30 minutes in a $4^{\circ} \mathrm{C}$ cold room (see Note 6).

15. Pre-chill the $1 \mathrm{X}$ TAE for electrophoresis by filling the electrophoresis chamber and allowing to chill in $4^{\circ} \mathrm{C}$ cold room. 
16. After restriction digest has completed, treat the target plasmid with 5 units of Antarctic phosphatase for 15 minutes at $37^{\circ} \mathrm{C}$ (see Note 7).

17. Add $15 \mu \mathrm{l}$ of $6 \mathrm{x}$ loading dye to each restriction digest and gently mix.

18. Carefully lower low melting point agarose gel into the electrophoresis chamber (see Note 8).

19. Load the full restriction digest into gel.

20. Load $6 \mu \mathrm{l}$ of GeneRule $1 \mathrm{~kb}$ DNA ladder into the flanking wells of the gel.

21. Run the gel at $100 \mathrm{~V}$ in the cold room until the lower band of the loading dye is at the bottom of the gel.

22. Carefully move the gel to a glass dish and stain with $0.5 \mu \mathrm{g} / \mathrm{ml}$ ethidium bromide for 30 minutes.

23. Visualize under UV light and estimate the DNA concentration by comparing the intensity of the ladder to sample bands according to manufacturers instructions.

24. Excise the uppermost telomere band with a fresh scalpel and place into a $1.7 \mathrm{~mL}$ tube (Figure 28).

25. Excise the target plasmid backbone with a fresh scalpel and place into a $1.7 \mathrm{~mL}$ tube (Figure 28). 


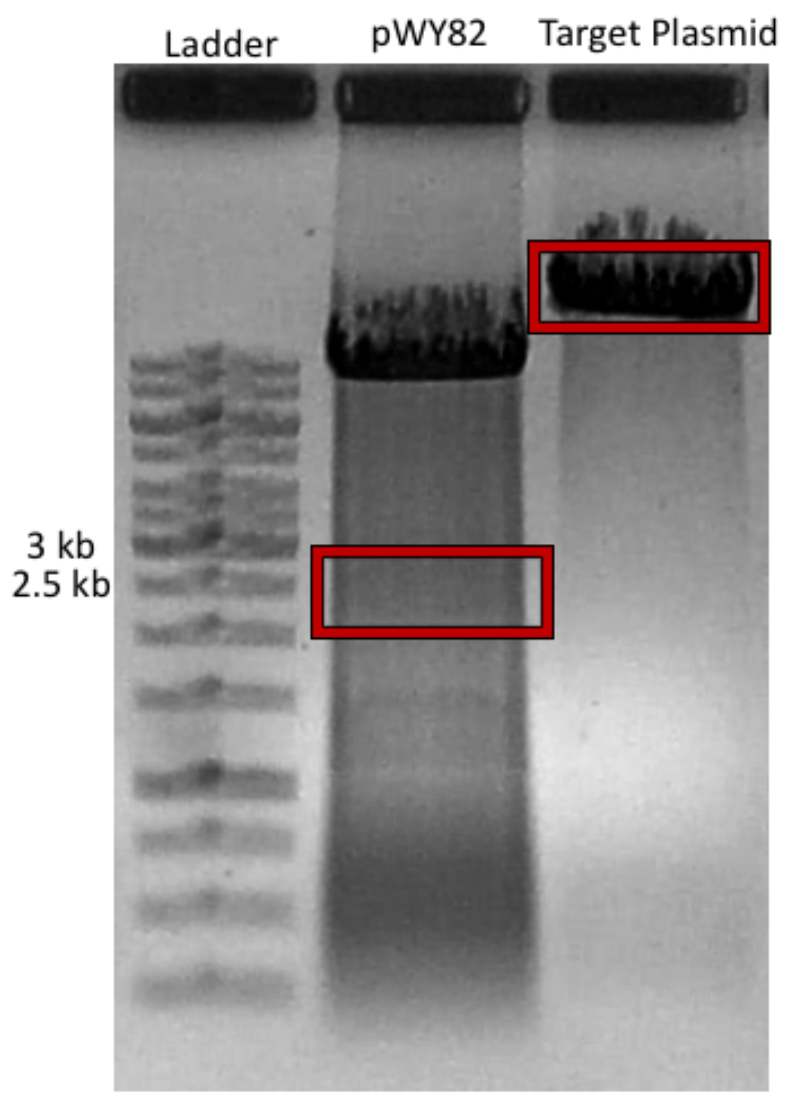

Figure 28 Example Gel Used for Telomere Ligation.

The boxed sections were removed and used for ligation.

26. In order to remove salts from the agarose slice add $1 \mathrm{~mL}$ of nuclease free water to each tube and place at $4^{\circ} \mathrm{C}$ overnight.

27. Completely remove water from each tube and place in a $70^{\circ} \mathrm{C}$ water bath.

28. Flick tubes every minute until gel has completely melted.

29. Once agarose has melted ( $\sim 5$ minutes), move to a $37^{\circ} \mathrm{C}$ water bath.

30. Allow gel fragments to cool to $37^{\circ} \mathrm{C}$, about 5 minutes.

31. Prepare ligation mixture in a $1.7 \mathrm{~mL}$ tube: 


\begin{tabular}{|l|l|}
\hline Ligase Buffer & $5 \mu \mathrm{l}$ \\
\hline Target Plasmid Gel Fragment & $100 \mathrm{ng}$ \\
\hline Ligase & $1 \mu \mathrm{l}(400$ units $)$ \\
\hline Telomere Gel Fragment & To $50 \mu \mathrm{l}$ \\
\hline
\end{tabular}

32. Once each component has been added, quickly mix by pipetting before ligation re-solidifies.

33. Incubate the solidified ligation overnight at room temperature.

34. Remove salts by incubating ligation in $1 \mathrm{~mL}$ of nuclease free water for 15 minutes.

35. Flick tube until gel is floating in water.

36. While ligation is dialyzing, begin to thaw Stbl4 cells on ice.

37. Replace water with $50 \mu \mathrm{l}$ of fresh nuclease free water and place in a $70^{\circ} \mathrm{C}$ water bath.

38. Flick tube every minute until completely melted.

39. Add $2 \mu \mathrm{l}$ of ligation to $40 \mu \mathrm{l}$ of Stbl4 cells and electroporate according to manufacturers instructions.

40. Resuspend cells in $900 \mu \mathrm{l}$ of S.O.C media and shake in $15 \mathrm{~mL}$ culture tubes at $30^{\circ} \mathrm{C}$ for 1.5 hours at $250 \mathrm{rpm}$.

41. Plate $100 \mu \mathrm{l}$ of transformation onto LB plates with appropriate antibiotics.

42. Incubate plates at $30^{\circ} \mathrm{C}$ (see Note 9).

43. Screen colonies via colony PCR or colony hybridization (see Note 10). 
44. Confirm telomere orientation using standard sequencing methods (see Note 11).

\subsubsection{Screening potential colonies for telomere size}

1. Prepare a starter culture by inoculating $3 \mathrm{~mL}$ of $2 X Y T$ liquid media cultures with the appropriate antibiotics of each colony to be tested, pWY82 for a positive control, and empty target vector as a negative control (see Note 12).

2. Shake at $250 \mathrm{rpm}$ for 8 hours at $30^{\circ} \mathrm{C}$.

3. Inoculate fresh $5 \mathrm{~mL} 2 \mathrm{XYT}$ liquid cultures with appropriate antibiotics and $100 \mu \mathrm{l}$ of starter cultures.

4. Shake cultures at $250 \mathrm{rpm}$ for $12-18$ hours at $30^{\circ} \mathrm{C}$.

5. Extract $4 \mathrm{~mL}$ of each culture with the QIAprep Spin Miniprep Kit (Qiagen) and elute with $40 \mu \mathrm{l}$ of $50^{\circ} \mathrm{C}$ nuclease free water.

6. Estimate concentration of each extraction using a nanodrop spectrophotometer.

7. Digest $1 \mu \mathrm{g}$ of each plasmid extraction with restriction enzymes that flank the telomere insert as closely as possible in a $50 \mu$ reaction volume.

8. Pour a $1 \%(w / v) 1 \times$ TAE agarose gel and insert a comb large enough to contain a $60 \mu$ volume and allow to solidify at room temperature.

9. Add $10 \mu \mathrm{l}$ of $6 \mathrm{x}$ loading dye to each restriction digest once completed. 10. Mix thoroughly by pipetting. 
11. Load each digest into agarose gel and run at $100 \mathrm{~V}$ in $1 \mathrm{x}$ TAE.

12. Stain gel in $0.5 \mu \mathrm{g} / \mathrm{mL}$ ethidium bromide solution for 30 minutes.

13. Visualize with UV light to confirm successful digest.

14. Transfer to a nitrocellulose membrane by Southern transfer (Green and Sambrook, 2012).

15. Follow Southern hybridization protocol (Green and Sambrook, 2012) using the radiolabeled oligonucleotide (TTTAGGG) 10

16. Compare the signal to the size standard to estimate the insert size.

\subsection{Production of Telomere Fragments via Polymerase Chain Reaction}

1. PCR Reaction Assembly (see Note 13):

The following PCR reaction has been assembled using LongAmp Taq DNA polymerase from New England Biolabs (volumes listed are per reaction volumes) (see Note 14):

\begin{tabular}{|r|l|l|}
\hline Nuclease Free Water: & 12 microliters & 24 \\
5x LongAmp Taq Buffer: & 4 microliters & microliters \\
Forward Telomere & 0.5 microliters & 8 microliters \\
$\underline{\text { Primer: }}$ & 0.5 microliters & 1 microliter \\
$\underline{\text { Reverse Telomere }}$ & 2 microliters & 1 microliter \\
$\underline{\text { Primer: }}$ & 1 microliter & 4 microliters \\
$\underline{\text { 10mM DNTPs: }}$ & & 2 microliters \\
\hline
\end{tabular}


2. Use the following thermocycler protocol to perform PCR with the assembled reaction mixtures.

\begin{tabular}{|c|c|}
\hline \multicolumn{2}{|c|}{$\frac{\text { Thermocycler Protocol }}{\text { for Telomere }}$} \\
\hline $\begin{array}{l}98^{\circ} \mathrm{C} 10 \mathrm{sec} \\
55^{\circ} \mathrm{C} 20 \mathrm{sec} \\
72{ }^{\circ} \mathrm{C} \quad 5 \mathrm{sec}\end{array}$ & Repeat 5x \\
\hline $\begin{array}{l}98^{\circ} \mathrm{C} 20 \mathrm{sec} \\
55^{\circ} \mathrm{C} 20 \mathrm{sec} \\
72{ }^{\circ} \mathrm{C} \quad 5 \mathrm{sec}\end{array}$ & Repeat 5x \\
\hline $\begin{array}{l}98^{\circ} \mathrm{C} 20 \mathrm{sec} \\
55^{\circ} \mathrm{C} 20 \mathrm{sec} \\
72{ }^{\circ} \mathrm{C} 10 \mathrm{sec}\end{array}$ & Repeat 5x \\
\hline $\begin{array}{l}98^{\circ} \mathrm{C} 20 \mathrm{sec} \\
62{ }^{\circ} \mathrm{C} 20 \mathrm{sec} \\
72{ }^{\circ} \mathrm{C} 30 \mathrm{sec}\end{array}$ & Repeat 5x \\
\hline $\begin{array}{l}72{ }^{\circ} \mathrm{C} 5 \\
\min .\end{array}$ & \\
\hline $4^{\circ} \mathrm{C} \infty$ & \\
\hline
\end{tabular}

3. After completion of thermocycler protocol, gel electrophoresis should be performed. Load the entire volume of each sample into the gel, as DNA gel extraction will be performed following visualization by gel electrophoresis. Example protocol for gel electrophoresis can be found in 
Sambrook and Russell 2001. Telomere DNA will appear as "smears", such as those visible in Figure 28.

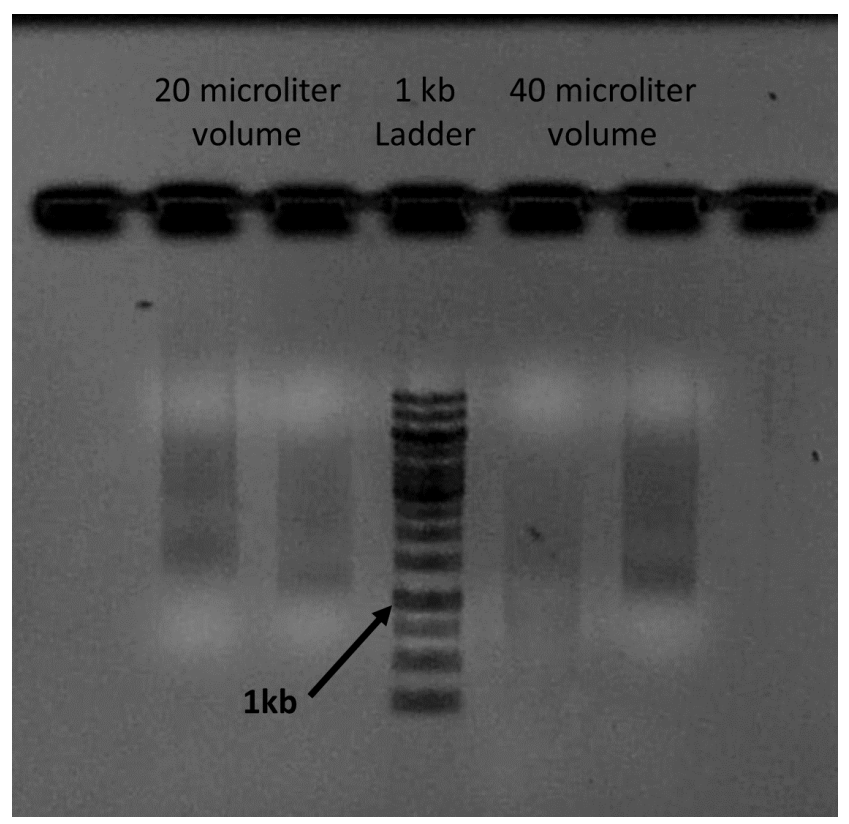

Figure 29 Telomere PCR Example Using 20 ul and 40 ul Reaction Volumes.

The "smears" in the sample lanes are indicative of telomere DNA conglomerates formed of various sizes.

4. DNA gel extraction is performed using a kit such as Wizard® SV Gel and PCR Clean-Up System. Excise the gel band corresponding to the size of telomere DNA desired. Follow manufacturer's instructions for extraction of DNA from the gel piece (see Note 15).

5. The resulting DNA from the gel extraction can be used in a cobombardment with a transgene.

\subsection{Transgene Delivery into Plants with Telomere Arrays}


1. Insert transgene and telomere sequence into target organism following standard transformation protocols (see Note 16).

2. Screen transgenic plants for minichromosomes using fluorescence in situ hybridization (see Note 17).

\section{Notes}

1. This vector should have cloning sites that are compatible with those bordering the telomere sequence in plasmid pWY82.

2. This plasmid contains $\sim 2.6 \mathrm{~kb}$ of telomere sequence and can be obtained through AddGene (addgene.org Plasmid\# 65721).

3. A working stock of these primers should be prepared at a 1.25 micromolar/microliter concentration.

4. pWY82 is maintained in STBL2 cells to stabilize the repeated sequences. These cells are slow growing and often will not be visible on plates or in culture for 24 hours.

5. Telomere sequences are unstable, and often will form secondary structures when run in an agarose gel. In most cases, the telomere will appear as multiple bands, or a smear, in addition to the $2.6 \mathrm{~kb}$ full telomere band (Figure 1).

6. A comb must be used that is large enough to fit $65 \mu$ l.

7. If restriction enzymes and Antarctic phosphatase are both purchased from the same manufacturer it is not usually necessary to clean up the restriction digest between reactions. 
8. Caution: low melting point gels must be handled extremely carefully as they are fragile.

9. As cells must be grown at $30^{\circ} \mathrm{C}$ it often takes 48 hours for colonies to appear.

10. Though the technique requires radiation safety training, Southern Hybridization (Sambrook et al., 1989) is the recommended procedure for screening colonies for telomere. In our experience, hundreds of colonies can be screened at once, and using a labeled oligonucleotide probe of (TTTAGGG) ${ }_{10}$ in either the $5^{\prime}$ or $3^{\prime}$ direction is extremely sensitive.

11. Screening via colony PCR has been used in our lab; however, it is not as effective as Southern Hybridization. While most colony PCR procedures will suggest choosing primers that will cause amplification across the inserted DNA, this is not possible with telomere as the complex repeats will disrupt amplification. As a result, the PCR will fail and give false negative results. Consequently, there are two options for colony PCR to detect telomere repeat insertion. First, reliable primers can be used flanking the insertion site and a blank band can be considered a positive insertion. While this method has been successful, it relies on the polymerase failing during amplification. The second option relies on a primer in the target plasmid, and another on the sequence adjacent to the telomere repeats that will also be inserted into the target plasmid during ligation. This method has also been successful; however, because the 
primers originate in different plasmids, the user is not able to have a positive control.

12. Due to the complexity of the telomere repeats, sequencing will often fail after a few hundred base pairs. While this procedure can not determine the complete length of inserted telomere, it is helpful to ensure that the ligation was completed in the correct orientation.

13. Prepare in the morning so that full cultures can be started in the evening and allowed to grow overnight.

14. A proofreading Taq polymerase should be used, for example, LongAmp Taq DNA Polymerase from New England Biolabs.

15. Make several reactions so that plenty of DNA can be obtained from DNA gel extraction. If using a different proofreading Taq, follow manufacturer's instructions for volume of buffer, Taq, and DNTP's if required.

16. Performing DNA gel extraction will greatly reduce the amount of DNA in each sample. For this reason, it is recommended that multiple thermocycler reactions are prepared to insure enough DNA is obtained. Nuclease-free water may be used for elution of DNA from the kit's column to ensure no interference with ligation reactions.

17. Agrobacterium transformation and particle bombardment are the two methods that have been successfully used to induce telomere-mediated truncation in maize (Gaeta et al., 2013; Yu et al., 2006). Particle bombardment can accomplish telomere-mediated truncation by bombarding in a transgene with attached telomere or separately by 
cobombardment of the transgene with free telomere arrays (Gaeta et al., 2013). The standard protocols for both particle bombardment and Agrobacterium transformation are unchanged by inclusion of telomere arrays and should be followed for the organism of interest.

18. Visualization of the genome using fluorescence in situ hybridization (FISH) is useful for finding the general location of chromosomal insertions or truncations (Gaeta et al., 2013; Yu et al., 2006). If the inserted transgene is small, a protocol has been established for finding small targets in the maize genome using FISH (Lamb et al., 2007), and should be applicable to other organisms. Minichromosomes can be distinguished from standard transgene inserts as the transgene will be located on the tip of a chromosome arm, and the chromosome is usually distinctly shorter when compared to its homologue.

\section{Acknowledgement}

This work was supported by NSF grant IOS-1339198 from the Plant Genome Program.

\section{References}

Gaeta, R., Masonbrink, R., Krishnaswamy, L., Zhao, C., and Birchler, J. (2012). Synthetic Chromosome Platforms in Plants. Annual Reviews of Plant Biology 63, 307-330. 
Gaeta, R.T., Masonbrink, R.E., Zhao, C., Sanyal, A., Krishnaswamy, L., and Birchler, J.A. (2013). In vivo modification of a maize engineered minichromosome. Chromosoma 122, 221-232.

Green, M.R., and Sambrook, J. (2012). Molecular Cloning: A Laboratory Manual (New York: Cold Spring Harbor Laboratory Press).

Ijdo, J.W., Wells, R. a, Baldini, a, and Reeders, S.T. (1991). Improved telomere detection using a telomere repeat probe (TTAGGG)n generated by PCR. Nucleic Acids Research 19, 4780.

Lamb, J.C., Danilova, T., Bauer, M.J., Meyer, J.M., Holland, J.J., Jensen, M.D., and Birchler, J. a (2007). Single-gene detection and karyotyping using small-target fluorescence in situ hybridization on maize somatic chromosomes. Genetics 175, 1047-1058.

Sambrook, J., Fritsch, E.F., and Maniatis, T. (1989). Molecular Cloning: A Laboratory Manual (Cold Spring Harbor Laboratory Press).

Southern, E.M. (1975). Detection of specific sequences among DNA fragments separated by gel electrophoresis. Journal of Molecular Biology 98, 503517.

Yu, W., Lamb, J.C., Han, F., and Birchler, J. a (2006). Telomere-mediated chromosomal truncation in maize. Proceedings of the National Academy of Sciences 103, 17331-17336.

Yu, W., Han, F., and Birchler, J. a (2007). Engineered minichromosomes in plants. Current Opinion in Biotechnology 18, 425-431. 


\title{
Chapter 5 Producing a BiBAC Vector for the Production of
}

\section{Minichromosomes in Maize}

\begin{abstract}
Minichromosomes are small chromosomes that are maintained independently of the endogenous chromosome set and contain an introduced transgene. In maize, minichromosomes are produced by truncating the supernumerary B chromosome, which removes the B chromosome accumulation mechanism and inserts a transgene. Minichromosomes to date have relied on standard plasmids, which only allow for a small amount of genetic cargo to be transferred each time a minichromosome is produced. In this work, a binary bacterial artificial chromosome (BiBAC) vector is constructed for the purposes of producing minichromosomes. The vector contains a multiple cloning site with rare-base cutters, a removable bialaphos selection gene, an attachment site for future addition with $\Phi C 31$ integrase, and telomere repeats at the right border. The vector is demonstrated to be able to contain at least $128 \mathrm{~kb}$ of insert DNA, and is capable of transferring a large genetic cargo to maize cells for regeneration.
\end{abstract}

\section{Introduction}

Minichromosomes are autonomous chromosomes that exist independently of the normal chromosome set. To be maintained, this means that they must contain a centromere, telomere, origin or replication, and a selectable marker for 
successful recovery and maintenance through plant transformation. In yeast, where the first artificial chromosome was assembled, these different sequences can be assembled together to form a functional minichromosome (Murray and Szostak, 1983). However, in plants this is not as simple as the centromere is not maintained by a simple sequence repeat. This can be observed by the numerous inactive centromeres that have been found that contain the centromere sequences usually attributed to kinetochore formation but do not result in kinetochore assembly (Gao et al., 2011; Han et al., 2006, 2009). Additionally, the introduction of centromeric repeats to rice did not result in kinetochore assembly (Phan et al., 2007), adding to the evidence that centromere formation is epigenetically regulated. The epigenetic nature of plant centromeres means that to produce a minichromosome one must take advantage of an already functional centromere. Utilizing such a chromosome also has the added advantage of the origin of replication and telomere sequences already being present as well. As a result, fewer sequences must be introduced to produce a new minichromosome.

To produce a minichromosome from an existing chromosome, telomeremediated chromosomal truncation is used. In telomere-mediated truncation, telomere sequences are introduced with a transgene and selection marker. As part of transgene integration, a double-stranded break must be produced in the target genome to allow for the new sequences to be introduced. Normally, this break is reannealed by the cell machinery, integrating the newly introduced transgene. In telomere-mediated truncation, however, the newly introduced telomere sequences are recognized as a new chromosomal terminus and the cell 
machinery extends the telomere sequences into an active telomere. As a result, sequences distal to this insertion point will be lost in the process. As a result, the random nature of transgene integration means that any chromosome can be susceptible to truncation during the transformation process. While the loss of genes will be selected against during regeneration, the process will cause a reduction in transformation efficiency, a process which is already inefficient. Additionally, as there is no control over orientation of an integration event, it is possible for a truncation to occur with the transgene itself being on the lost fragment.

As telomere truncation will cause a loss of genetic material, endogenous chromosomes are not the best target for minichromosome production. In maize, the supernumerary $\mathrm{B}$ chromosome is the most widely utilized target. The $\mathrm{B}$ chromosome is an additional chromosome to the normal chromosomal set in maize that does not pair or recombine with the normal A chromosomes (Jones and Houben, 2003). The B chromosome is maintained by an accumulation mechanism that causes non-disjunction at the second pollen mitosis and preferential fertilization of the egg by sperm containing B chromosomes. The genes responsible for this accumulation mechanism are trans-acting and located at the distal tip of the B chromosome long arm. There are no vital genes known to exist on the B chromosome, that makes it an excellent target for minichromosome production. Additionally, as telomere-mediated truncation will result in the loss of genetic material, truncation of the B chromosome long arm will most likely remove the accumulation mechanism genes. The resulting 
minichromosome would allow for stable transmission of the introduced transgene, but transgene copy number could also be manipulated by introducing intact B chromosomes containing the trans-acting accumulation mechanism.

Minichromosomes were first developed in maize in 2006 (Yu et al., 2006) by introducing a construct containing $2.6 \mathrm{~kb}$ of telomere sequence at the right border of an Agrobacterium compatible vector. Telomere-mediated truncation has also since been demonstrated through the use of particle bombardment as well (Yu et al., 2007b). Whether minichromosomes are produced through Agrobacterium tumefaciens or particle bombardment, they are still limited by the amount of DNA originally introduced. While modifications to minichromosomes have been demonstrated (Gaeta et al., 2013; Yu et al., 2007b), they have thus far not been able to introduce new genetic material to an already produced minichromosome. As a result, researchers must produce new minichromosomes for each additional transgene desired, and those minichromosomes maintained together through crossing schemes. This is problematic as the transformation process in maize is a low efficiency process, which is only reduced by telomere truncation.

To alleviate this problem, a study was performed to use a binary bacterial artificial chromosome (BiBAC) to produce a minichromosome (Vega et al., 2008a). BiBAC vectors contain the $\mathrm{F}$ origin of replication originally utilized in bacterial artificial chromosomes, which allows for maintenance of at least $300 \mathrm{~kb}$ of total DNA (Shizuya et al., 1992). BiBAC vectors also contain the necessary genes for maintenance in Agrobacterium tumefaciens as well as E. coli. The 
binary nature of the plasmids means they can be used to transfer large genetic fragments to plants. Indeed, $150 \mathrm{~kb}$ of DNA has been successfully transferred to tobacco plants using these plasmids (Hamilton et al., 1996). A BiBAC plasmid was modified by Vega et al (2008) to include a selection marker, large DNA fragment from yeast, and telomere sequences for the purposes of producing a minichromosome. After introduction of this plasmid to maize embryos, however, a minichromosome was not found despite multiple regenerated plants containing the construct. Successful recovery of transgenic plants indicates that the BiBAC vector was fully capable of transferring large DNA fragments to maize embryos, suggesting that the reason for the lack of minichromosomes was a result of other circumstances. It is important to note that transgenic plants were not screened until the $\mathrm{T} 1$ generation. This is significant as truncations that may have occurred on A chromosomes would most likely not be recovered unless on the very tip of the chromosome arm as truncations would have resulted in loss of necessary genetic material. More importantly, the telomere sequences introduced into the BiBAC vector were placed at the left border. This is contrary to the original minichromosome producing vectors, which contained telomere sequences at the right border. The lack of truncation with telomere sequences located at the left border suggests that the integration mechanism of T-DNA will not expose telomere sequences for recognition by capping machinery unless the repeats are located at the right border. While the precise mechanism of T-DNA integration is not known (Gelvin, 2010), there are numerous proteins and complexes involved with T-DNA incorporation that could potentially interfere with end capping 
machinery during the process. As there have been many instances of minichromosomes produced with vectors containing telomere sequences at the right border, and none reported that contain these sequences at the left border, it is likely that this is the cause of the lack of minichromosomes produced with the earlier mentioned BiBAC vector.

In this work, a BiBAC vector was created to determine if it is indeed possible to produce minichromosomes while transferring a large DNA cargo. A BiBAC vector was created that contains the bialaphos selectable marker, multiple cloning site for DNA cargo insertion, and telomere sequences located at the right border. Using this vector, large segments of DNA from yeast were inserted into the multiple cloning site for proof-of-concept experiments. After screening for telomere insert size, two of these constructs were used for maize transformation. After extensive transformation experiments, only one event was found that contained the full T-DNA insert. This event was screened and found on the distal tip of the long arm of chromosome 1 . While the vector is confirmed to have the capability of transferring large cargo to maize cells, more work is required to determine if it is truly capable of minichromosome production.

\section{Materials and Methods}

\section{BiBAC Vector Construction}

The BiBAC vector pJV06 (Vega et al., 2008a) was used as the starting material for BiBAC vector construction as this vector was previously demonstrated as having the necessary components for large-cargo DNA transfer 
in maize. BiBAC plasmid DNA was obtained by first growing $5 \mathrm{~mL}$ cultures in $2 \mathrm{xYT}$ medium for 8 hours at $37^{\circ} \mathrm{C} .1 \mathrm{~mL}$ of starter culture was then used to spike $2250 \mathrm{~mL}$ cultures of 2xYT media in 1 liter flasks. Cultures were grown overnight at $37^{\circ} \mathrm{C}$ shaking at $250 \mathrm{rpm}$. Plasmid extraction was carried out using the NucleoBond Xtra Bac kit (Machery-Nagel) following the manufacturers instructions. Extracted DNA was resuspended in water and kept at $4^{\circ} \mathrm{C}$ for up to 1 month.

Plasmid pJV06 was modified by digesting with Pacl and inserting a PCR amplified fragment consisting of a Ubiquitin promoter, bialaphos selection gene flanked by loxP sites, nos terminator, and SPM TIR region from plasmid pBKSGZUS to create plasmid pNG1. pNG1 was modified by inserting a multiple cloning site for further cloning into an Ascl site to create pNG2. The selection cassette originally inserted into pJV06 was then replaced by digesting with $\mathrm{Pacl}$ and inserting a newly PCR amplified cassette from plasmid pPZPGLP1, which contains a removable bialaphos selection gene under the ubiquitin promoter, and attachment site attP for future integration with $\Phi C 31$ integrase. The newly created plasmid, pNG5 was then further modified by inserting a multiple cloning site containing rare-base cutters into the $\mathrm{Ascl}$ and Notl cut sites to create plasmid pNG6 (figure 30). Telomere was inserted into the Ascl and Swal sites of plasmid pNG6 via in-gel ligation (chapter 4). 


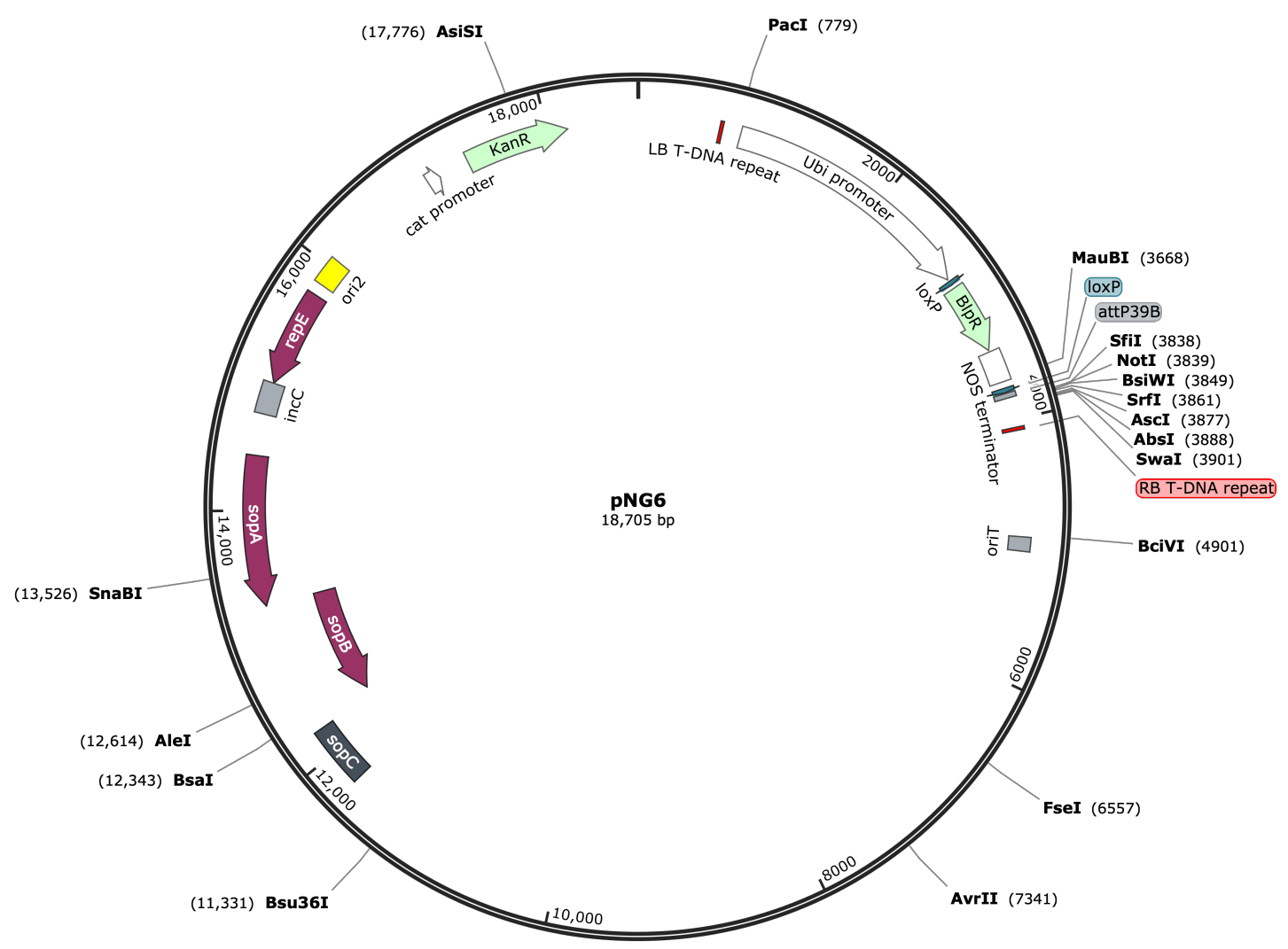

Figure 30 Plasmid pNG6

BiBAC Vector pNG6 contains a removable bialaphos selection gene (BlpR) under the maize ubiquitin promoter. The selection cassette is flanked by loxP sites for the removal of selection in the presence of Cre recombinase. Following the selection cassette is the minimal $39 \mathrm{bp}$ attachment site attP for future integration with the recombinase $\Phi С 31$ integrase. The attachment site is followed by a multiple cloning site containing rare-base cutter enzymes for insertion of large genetic fragments.

\section{Telomere Insertion and Verification}

Potential colonies of telomere insertion into plasmid pNG6 were first screened via colony PCR for telomere insertion. Primers BarNosSeq3 and WY82R were used for colony PCR to detect the presence of $\mathrm{pWY} 82$ sequences 
cloned into vector pNG6 with adjacent telomere sequences. Positive colonies were then grown in $5 \mathrm{~mL}$ cultures and extracted with the ZR BAC DNA Miniprep Kit (Zymo Research). The entire DNA extraction was then reduced in volume with a SpeedVac and subjected to restriction digest with $\mathrm{Fsel}$ and Ascl enzymes. The resulting digest was then separated on an agarose gel and subjected to Southern hybridization with a probe specific to the plant telomere sequence. Two colonies displayed signal in the hybridization, which were grown in $250 \mathrm{~mL}$ cultures and extracted with the NucleoBond Xtra Bac kit (Machery-Nagel). $10 \mu \mathrm{g}$ of plasmid DNA was digested with Fsel and Ascl enzymes and separated on an agarose gel (figure 31). To determine the size of telomere inserted into the colonies, the gel was subjected to Southern hybridization with a telomere probe (figure 32). Telomere orientation and further confirmation was provided by Sanger sequencing of the resultant colonies. 


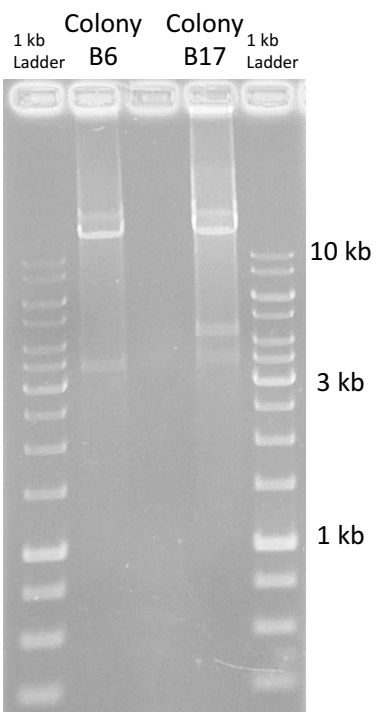

Figure 31 Digest pNG6 Colonies Containing Telomere

Agarose gel of restriction digest of colonies containing telomere repeat. Colonies were digested with $\mathrm{Fsel}$ and $\mathrm{Ascl}$ enzymes. Digest should yield a $16 \mathrm{~kb}$ fragment corresponding to the BiBAC backbone, and a fragment of 2680 bp plus telomere insertion size. Multiple bands present in lane 4 is indicative of telomere insertion as telomere repeats often form secondary structures in agarose gels. 


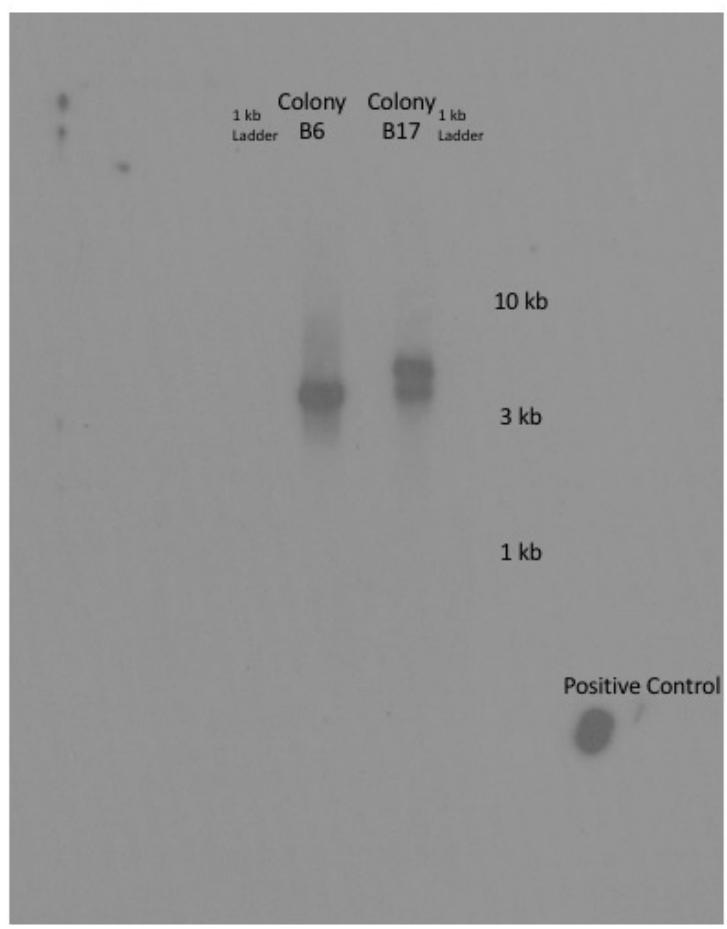

Figure 32 Telomere Southern Blot

Southern blot of pNG6 colonies with telomere insert. A radiolabeled telomere oligo was used to check for presence and size of telomere inserted into plasmid pNG6. An aliquot of pWY82 was placed onto the membrane as a positive control prior to blotting, and the DNA ladder served as a negative control. Overlaying the developed radioimage on the agarose gel image (Figure 31) provides a telomere size for colony B6 of $800 \mathrm{bp}$ and colony B17 of $1800 \mathrm{bp}$ when the full size of the excised DNA fragment is considered.

Following Southern hybridization and Sanger sequencing, two plasmids were found to contain telomere sequence, B6 and B17, with $800 \mathrm{bp}$ and $1800 \mathrm{bp}$ of telomere repeat, respectively. These plasmids were consequently named pNG6+T800 and pNG6+T1.8. As pNG6+T1.8 contained the largest amount of telomere repeat, it was chosen for further manipulations. 


\section{Insertion of Large DNA Cargo}

To determine if the developed BiBAC vector pNG6+T1.8 could transfer large DNA cargo while producing a minichromosome, it was necessary to insert DNA into the multiple cloning site. As the experiment would serve as a proof of concept, inert DNA that could easily be located with fluorescent in-situ hybridization was desired. As a result, yeast DNA, from strain BY4741, was chosen to create a range of $\mathrm{BiBAC}$ sizes. Restriction cloning with enzyme BsiW was performed by Intact Genomics, which generated 9 clones ranging in size from $58 \mathrm{~kb}$ to $149 \mathrm{~kb}$ (figure 33).

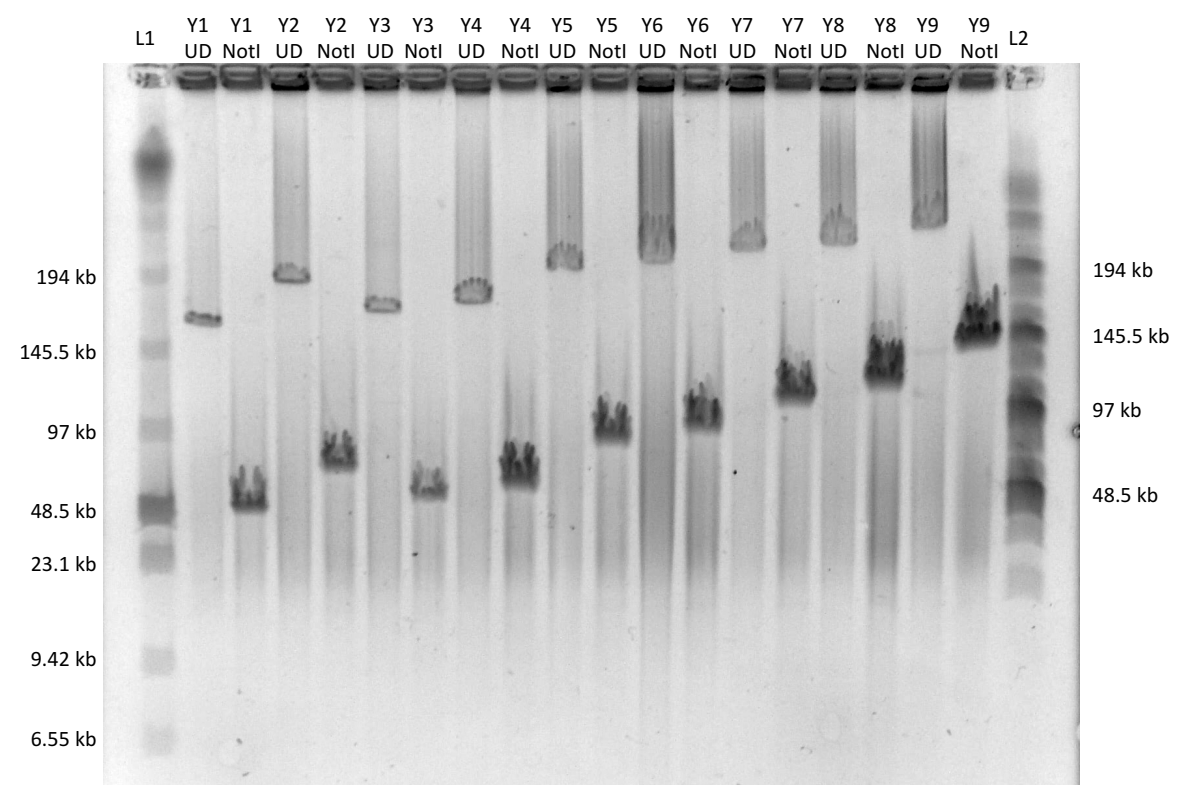

Figure 33 Pulsed Field Gel of Yeast BiBAC Colonies

Pulsed field gel demonstrating the size range of BiBAC colonies containing yeast DNA. Colonies were either run undigested (UD) or after digesting with enzyme Notl. $1 \mu \mathrm{g}$ of DNA was loaded into each lane for PFGE analysis. Ladder 1 (L1) is a low range PFGE marker (NEB) and ladder 2 (L2) is a mid range PFGE marker. 
To determine the exact amount of yeast DNA that was inserted into plasmid pNG6+T1.8, yeast colonies were sequenced on an Illumina MiSeq System. Cleaned reads were aligned to the yeast genome and visualized with the Integrative Genomics Viewer (Robinson et al., 2011) to determine the precise sequences inserted and size (figure 34).

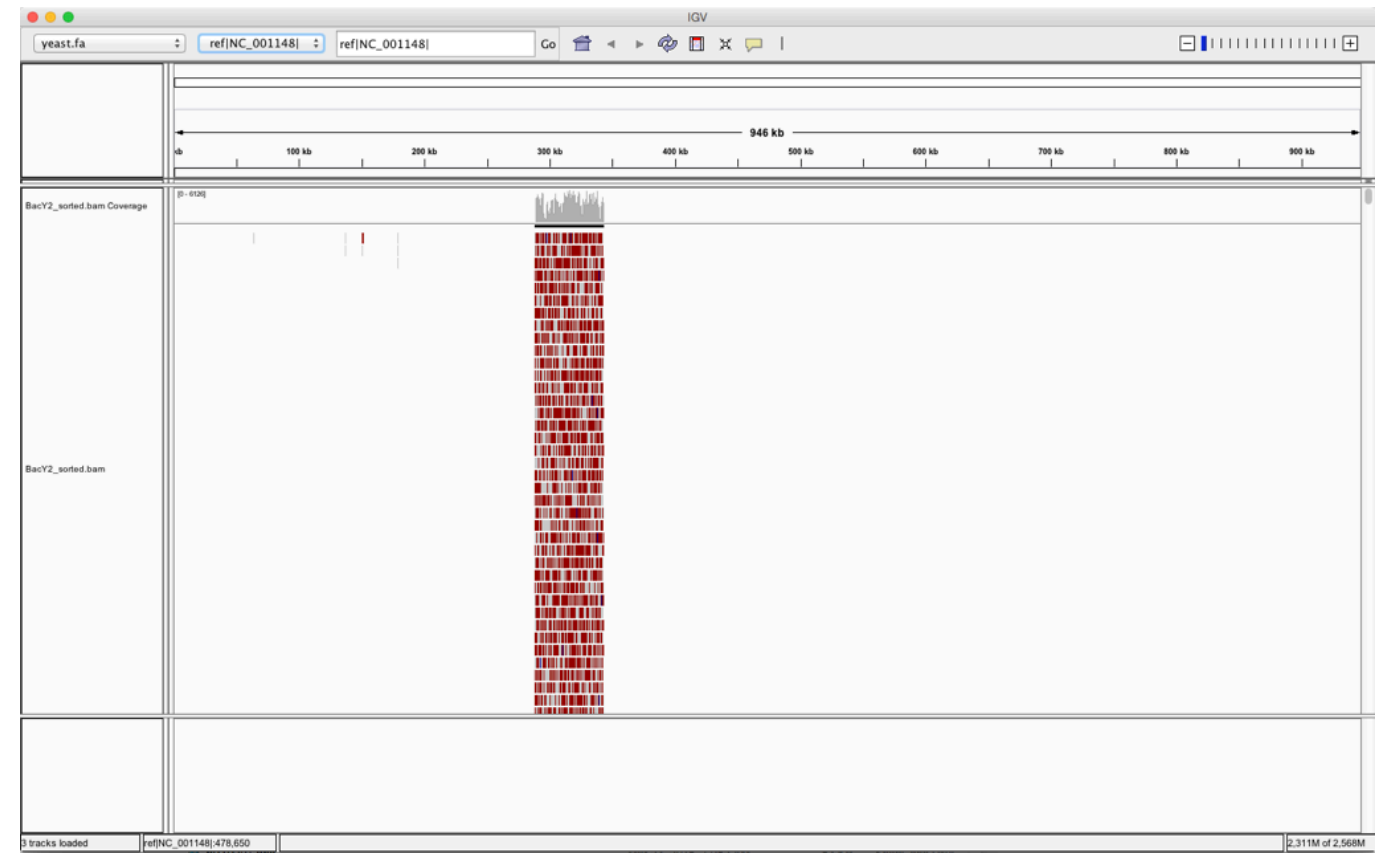

Figure 34 Example IGV Alignment of BiBAC Sequencing

Example IGV output of alignment of yeast chromosome 16 and BiBAC pNG6+T1.8_Y2. Red lines represent individual reads that map to the genomic location indicated.

As telomere sequences are unstable, colonies with yeast inserts were digested with enzyme Spel, run on an agarose gel (figure 35), and probed with a radiolabeled telomere oligo with a Southern hybridization. The developed 
exposure was compared to the original agarose gel to determine the size of telomere sequences present (figure 36).

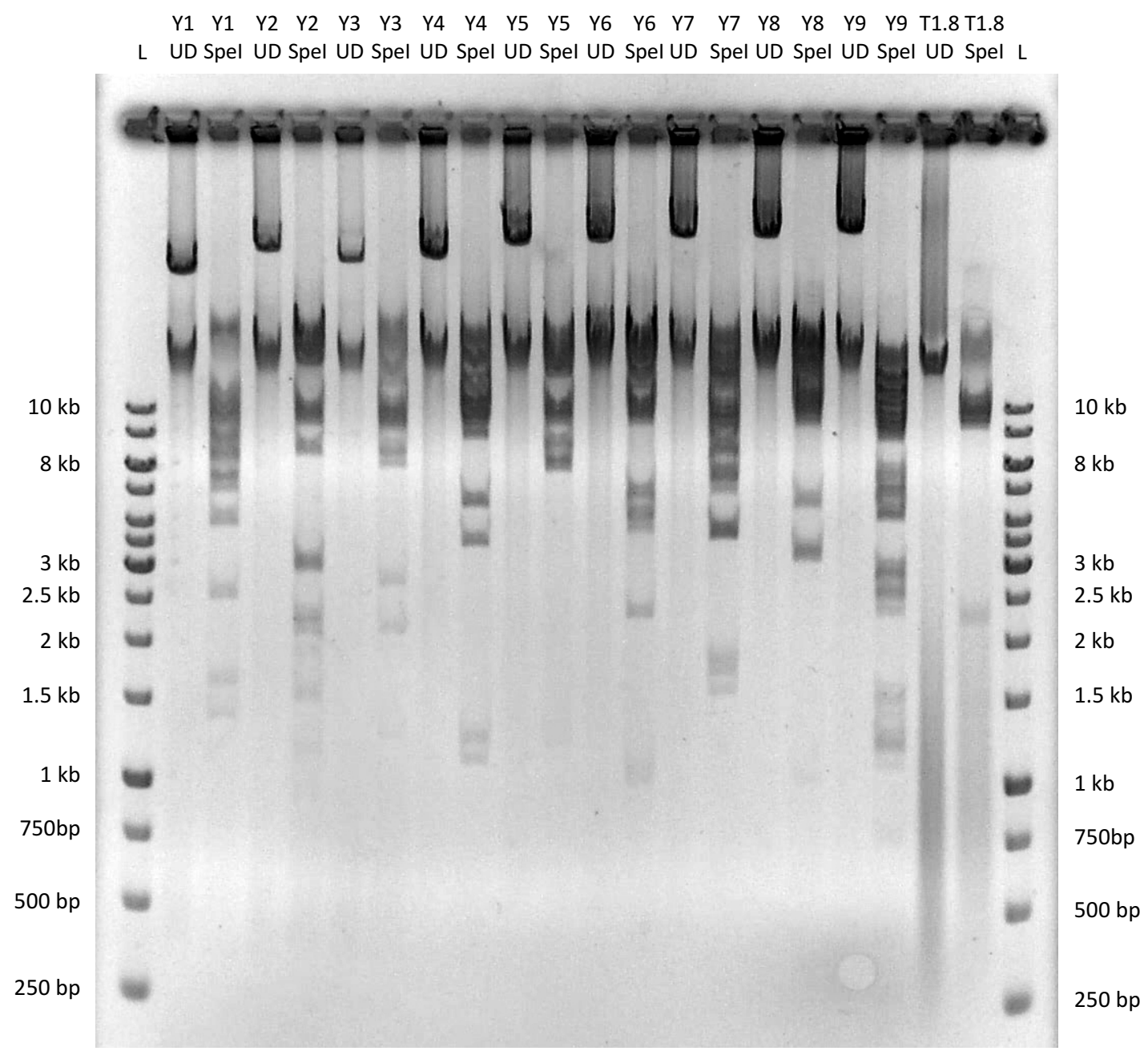

Figure 35 Digest of pNG6+T1.8 Yeast Colonies for Telomere Southern Hybridization

$1 \mu \mathrm{g}$ of each plasmid was loaded onto a standard agarose gel. Each plasmid was subjected to electrophoresis either as undigested (UD) or after digestion with Spel. 


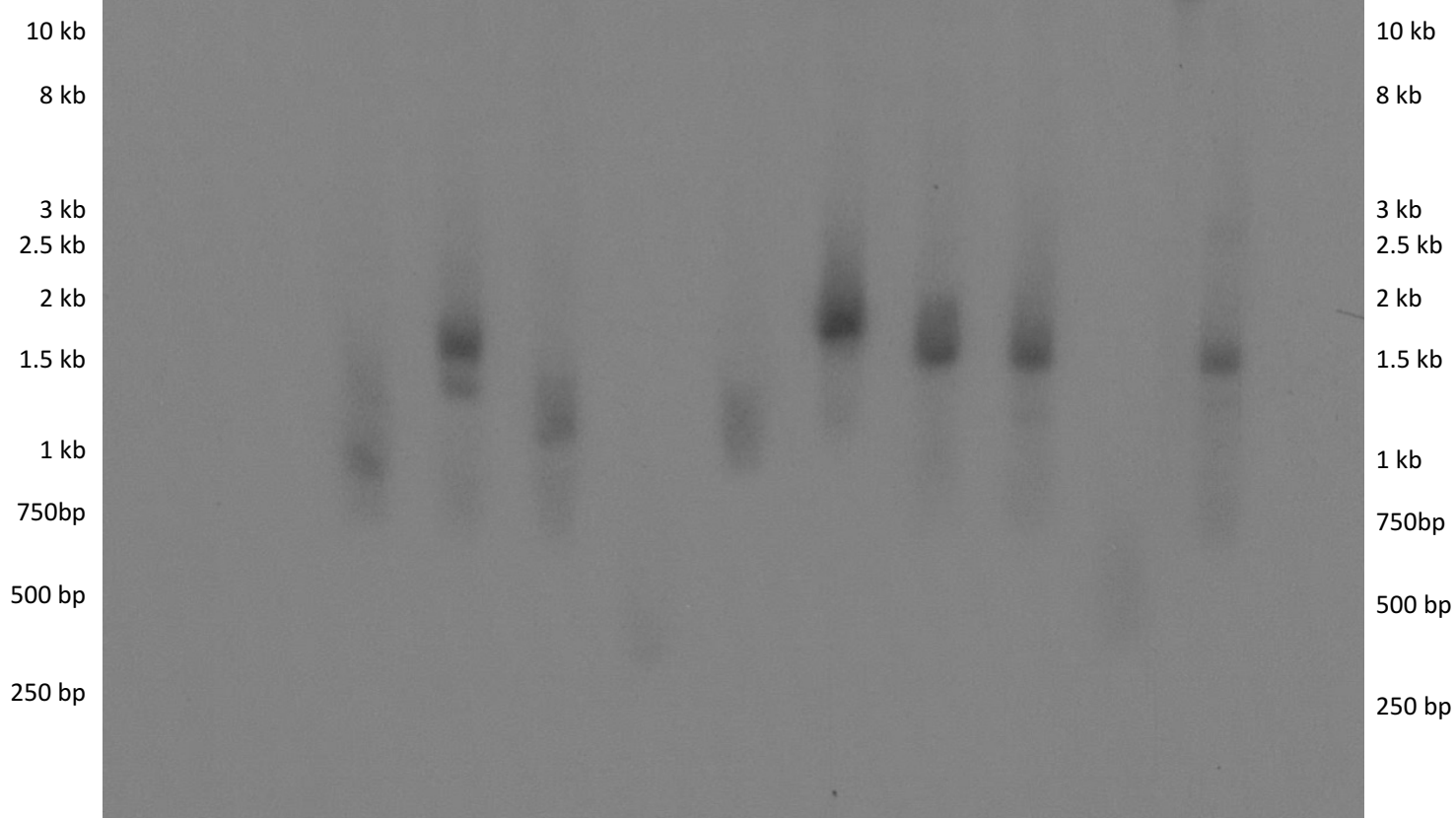

Figure 36 Southern Hybridization of pNG6+T1.8 Yeast Colonies

Southern blot of digested yeast colonies to determine amount of telomere repeat present.

Following digestion and Southern hybridization, telomere sizes could be estimated for each yeast containing colony. Combining this information with previous digestions and Illumina sequencing, table 15 was constructed to summarize the characterization of each plasmid. 


\begin{tabular}{|c|c|c|c|c|}
\hline $\begin{array}{c}\text { Plasmid Name (Yeast } \\
\text { Chromosome Number) }\end{array}$ & $\begin{array}{c}\text { Total Size } \\
\text { (bp) }\end{array}$ & $\begin{array}{c}\text { T-DNA } \\
\text { (bp) }\end{array}$ & $\begin{array}{c}\text { Yeast } \\
\text { Insert Size } \\
\text { (bp) }\end{array}$ & Telomere Size (bp) \\
\hline pNG6+T1.8_Y1 (VII) & 58,604 & 43,350 & 38,029 & 750 \\
\hline pNG6+T1.8_Y2 (XVI) & 83,013 & 67,759 & 62,442 & 1,300 \\
\hline pNG6+T1.8_Y3 (XVI) & 66,659 & 51,405 & 46,088 & 1,000 \\
\hline pNG6+T1.8_Y4 (IX) & 71,231 & 55,977 & 50,656 & 250 \\
\hline pNG6+T1.8_Y5 (VII) & 96,131 & 80,877 & 75,556 & 1,000 \\
\hline pNG6+T1.8_Y6 (XII) & 102,788 & 87,534 & 82,217 & 1,800 \\
\hline pNG6+T1.8_Y7 (V) & 117,594 & 102,340 & 97,023 & 1,500 \\
\hline pNG6+T1.8_Y8 (II) & 125,978 & 110,724 & 105,407 & 1,500 \\
\hline pNG6+T1.8_Y9 (XI) & 149,360 & 134,106 & 128,507 & 350 \\
\hline
\end{tabular}

Table 10 Summary of Yeast BiBAC Data

Summary of the known information about each yeast BiBAC plasmid. Each plasmid is listed followed by the total size of the BiBAC, number of nucleotides between the left and right border, size of the yeast DNA insert, and estimated telomere repeat size.

Following analysis, BiBACs range in size from $58 \mathrm{~kb}$ to $149 \mathrm{~kb}$, with telomere size ranging from $250 \mathrm{bp}$ to $1800 \mathrm{bp}$. For the purposes of the current study, two BiBAC plasmids were chosen for transformation, pNG6+T1.8_Y2 (Y2) and pNG6+T1.8_Y8 (Y8). These plasmids were chosen as they could demonstrate a range of T-DNA sizes, and contained a large amount of telomere repeats.

\section{BiBAC Transformation}

Maize transformation was performed using Agrobacterium tumefaciens (Vega et al., 2008b) on immature maize Hi-Il embryos. Embryos were a result of crossing maize $\mathrm{Hi}-\mathrm{Il}$ A line containing $\mathrm{B}$ chromosomes and $\mathrm{Hi}-\mathrm{Il}$ line $\mathrm{B}$. Embryos were collected 10 - 12 days after pollination when embryos measured between 1.5 and $2 \mathrm{~mm}$ in length. 
It has been suggested that Agrabacterium line LBA4404 results in a higher transformation efficiency when using BiBAC plasmids (He et al., 2010); however, the Agrobacterium line predominantly used in our hands is EHA105. As a result, BiBAC plasmids $\mathrm{Y} 2$ and $\mathrm{Y} 8$ were transferred to both LBA4404 and EHA105 to compare transformation efficiency. Additionally, helper plasmid pCH32 (Hamilton et al., 1996) was also introduced to Agrobacterium strains with the BiBAC plasmids. Plasmid pCH32 contains virE1, virE2, and virG genes, which are thought to assist with the transfer of large DNA strands during Agrobacterium infection.

After transformation of about 1500 embryos each of $\mathrm{Y} 2$ and $\mathrm{Y} 8$ with both EHA105 and LBA4404, it was determined that strain EHA105 would be used going forward. After multiple trials with LBA4404, and the use of multiple antibiotics, LBA4404 appeared to be resistant to antibiotic selection. As a result, callus was quickly killed due to overgrowth of Agrobacteria on plates. This was the case when new antibiotics were utilized at varying concentrations and with different modes of action. While it may be possible that either the BiBAC or helper plasmids were interacting with this particular Agrobacterium strain, this was not analyzed further.

Utilizing strain EHA105, transformation has been carried out over multiple growing seasons. For plasmid Y2, 6447 embryos have been infected with Agrobacterium, and for plasmid Y8 5875 embryos have been infected. In both cases, callus was found to be slow growing, and even in the event of a transformed plant was never friable and white. This may be the result of the 
helper plasmid, which increases the presence of virulence genes in the Agrobacterium cells. As a result of the delayed rate of callus growth, it was found to be necessary to delay the transfer of callus at each stage of transformation. For instance, callus was found to develop better when allowed to grow on resting media for 14-21 days as opposed to the normal 7-10 days.

\section{Analysis of Transformed Plant Y2-1}

After transformation, only one plant to date has been successfully regenerated as a result of BiBAC transformation. This plant, designated as plant Y2-1, was transformed from BiBAC pNG6+T1.8_Y2, which contains a T-DNA of approximately $67 \mathrm{~kb}$ with $1300 \mathrm{bp}$ of telomere repeat. The plant that resulted from transformation was slow growing, with multiple white sectors (figure 37). The plant produced few roots, and died only a few weeks after being moved to soil. 


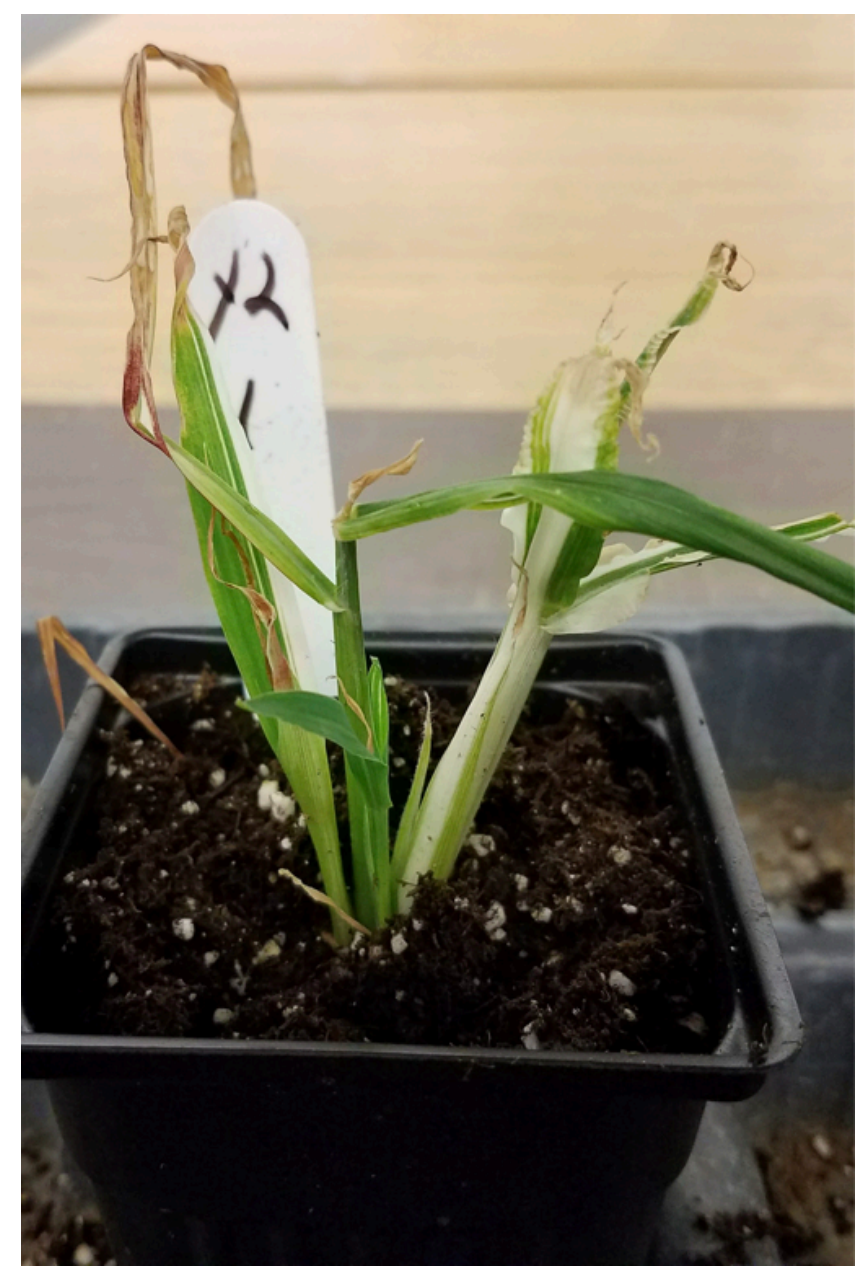

Figure 37 Plant Y2-1

Plant Y2-1 after transformation. The callus produced multiple shoots, which can be observed growing from a single location out of the soil. Each shoot contained white sectors throughout.

Soon after transferring the plantlet to soil, leaf tissue was taken for genotyping. Plant genomic DNA was extracted with the PowerPlant Pro DNA Isolation Kit (MoBio) following manufacturer's instructions. To determine if the full T-DNA had been transferred to the plant, PCR analysis was performed using primers that spanned the entire T-DNA of plasmid pNG6+T1.8_Y2 (figure 38). 


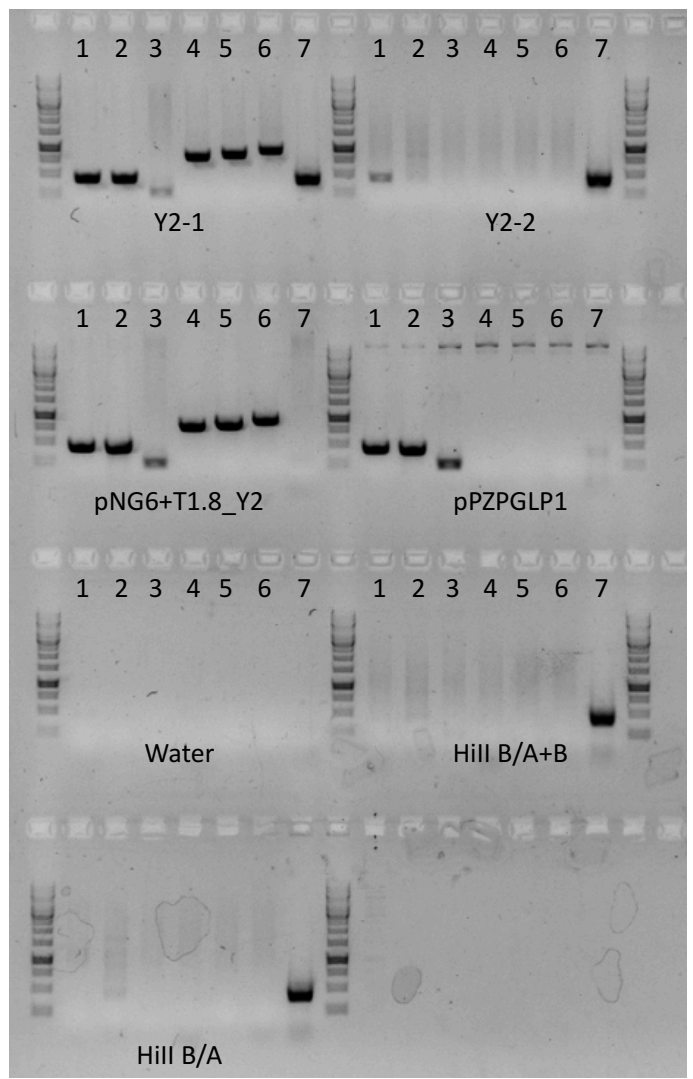

Figure 38 PCR of Plant Y2-1

PCR analysis of BiBAC transfer to plant Y2-1. PCR was performed on 7 different samples, plant Y2-1 which was thought to contain pNG6+T1.8_Y2 DNA, plant Y2-2 which was a false positive regenerated plant serving as a negative control, plasmid pNG6+T1.8_Y2 positive control DNA, pPZPGLP1 plasmid DNA which contains the same T-DNA sequences preceding the yeast DNA insertion, a negative control water sample, and DNA from normal Hi-Il plants containing $\mathrm{B}$ chromosomes $(\mathrm{Hi}-\mathrm{II} \mathrm{B} / \mathrm{A}+\mathrm{B})$ and without $\mathrm{B}$ chromosomes $(\mathrm{Hi}-\mathrm{II} \mathrm{B} / \mathrm{A})$. Primers were chosen that amplified fragments throughout the BiBAC T-DNA as follows; 1 - bialaphos resistance gene, 2 End of ubiquitin promoter into bialaphos resistance gene, 3 - End of bialaphos resistance gene into NOS terminator, 4 - Beginning of yeast DNA, 5 - Middle of yeast DNA, 6 - End of yeast DNA sequence, 7 - maize rubisco positive control. As the expected products were amplified across the T-DNA insert, it was determined that the full T-DNA was inserted into plant Y2-1. 
Following PCR analysis, roots from plant Y2-1 were collected for fluorescent in-situ hybridization (FISH). FISH analysis was performed following standard protocols (McCaw et al., 2016a, 2016b) using secondary roots following a week of growth in soil in a growth chamber. The first FISH image, probed with CentC and NOR probes, and stained with DAPI (figure 39), revealed multiple B chromosomes, with one appearing to be shortened. Following this experiment, a single-gene probe was developed that is specific to the Y2 yeast DNA (figure 40), that showed signal on the long arm of chromosome 1. 

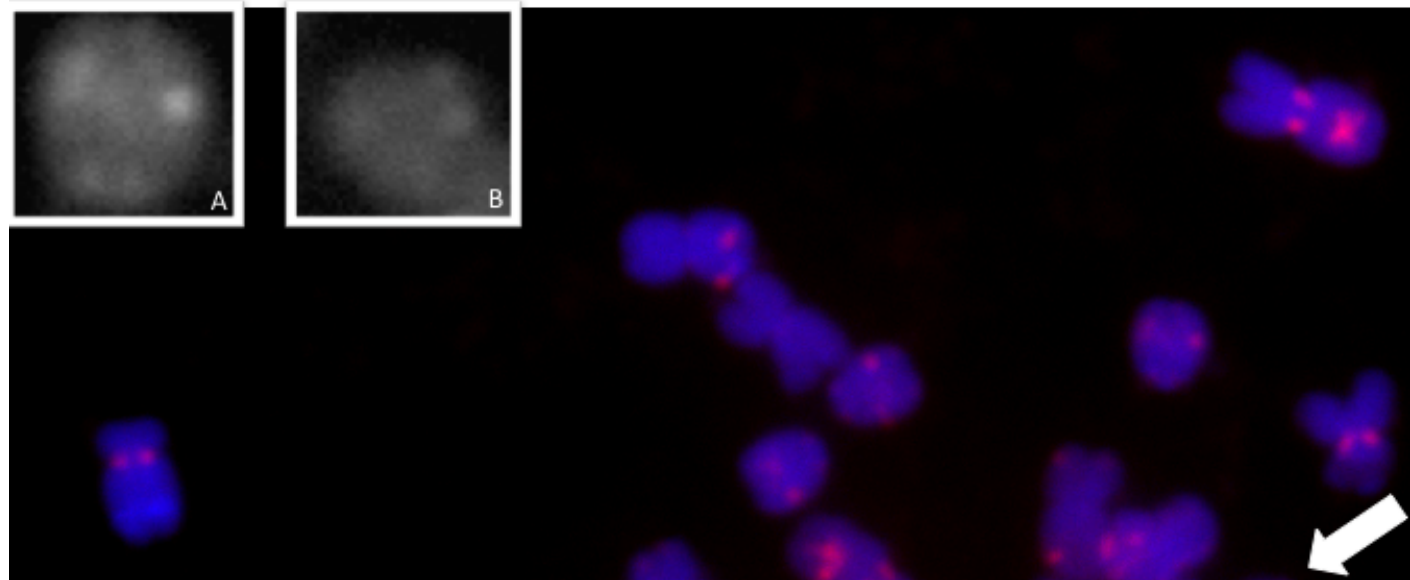

\section{Figure 39 FISH Image of Plant Y2-1}

FISH image from a secondary root of plant Y2-1. Slide was probed with CentC and NOR probes (Red) for chromosome identification, and DAPI stained (Blue). A B chromosome was found to be shortened (arrow) relative to other B chromosomes in the spread. (A) Normal B chromosome showing only the red channel. (B) Shortened B chromosome showing only the red channel. 


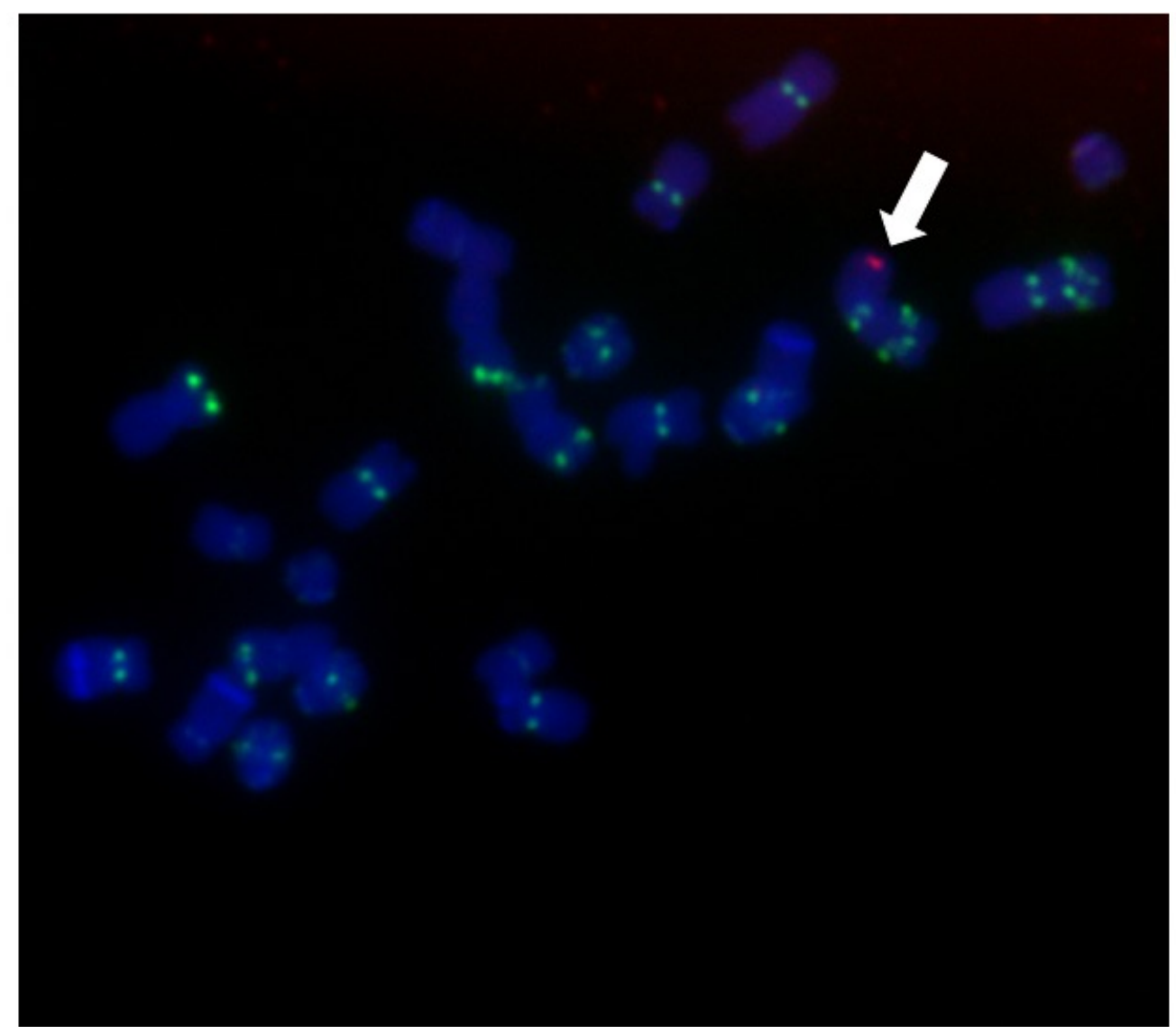

Figure 40 Single-Gene FISH of Plant Y2-1

FISH of plant Y2-1 with a single-gene probe (Red) specific to the yeast inserted into pNG6+T1.8_Y2 T-DNA. CentC and NOR probes (green) were also included for chromosome identification. The single-gene probe can be seen on the distal tip of the short arm of chromosome 1.

FISH images of $\mathrm{Y} 2-1$ suggest that the transgene is at the distal tip of chromosome 1. To determine where the transgene was located, TAIL-PCR was performed as described (Singer and Burke, 2003). Two rounds of PCR were performed utilizing nested primers, extending out from both the left and right borders. Products were only found that extended out from the left border. After the PCR products were Sanger sequenced, they were found to align with flanking 
right border sequences as expected, and then extend into a Ty1/Copia retroelement. As these elements are found throughout the maize genome, it is difficult to determine the exact location of the transgene insertion.

\section{Discussion}

Minichromosomes, autonomously maintained transgene vectors, allow for transgenes to be maintained separately from the main genome of interest. By integrating a transgene outside of the genome, issues such as linkage drag can be alleviated. Additionally, multiple minichromosomes could be combined with simple crossing techniques, allowing for traits to be easily combined. In maize, by truncating a B chromosome, there is no risk of loss of necessary genetic material, adding to the utility of the system.

To date, the vectors used for truncation have all been based on traditional cloning vectors. While these vectors can be easily manipulated, they usually rely on origins of replication that limit their overall size. As a result, the total plasmid size can not generally exceed about $20 \mathrm{~kb}$. When taking into account the plasmid backbone, this normally means that only a single gene can be included in a truncation vector. Consequently, multi-genic traits must be separately transformed and the resulting minichromosomes maintained with crossing schemes. Alternatively, mechanisms such as site-specific targeting (chapter 3) could be employed to add genetic content to an already existing minichromosome. 
To alleviate this problem, BiBAC vectors have been proposed to allow the integration of large pieces of DNA in a single truncation vector. BiBAC vectors, which utilize the $\mathrm{F}$ factor origin of replication, have been shown to transfer $150 \mathrm{~kb}$ of DNA to plants (Hamilton et al., 1996). By utilizing a large plasmid, researchers could obtain all of the benefits of a minichromosome, while also incorporating multi-genic traits as well. Additionally, by utilizing a telomere containing BiBAC for the production of BAC libraries, traits found in BAC library screens such as large genes or gene clusters would be immediately available for the production of minichromosomes.

In the current work, the ability of BiBAC vectors to produce minichromosomes was evaluated. A BiBAC vector containing telomere repeats, pNG6+T1.8, was produced that contained a removable selection gene, attachment site for future addition with $\Phi C 31$ integrase, and a multiple cloning site containing rare-base cutters. Using yeast DNA, this vector was demonstrated as having the capability of accepting an insert of at least $128 \mathrm{~kb}$. Additionally, the vector was shown to have the ability to transfer a large DNA cargo to maize cells, and successfully be selected during transformation.

The ability of the BIBAC vector to produce a minichromosome was inconclusive. This is mostly a result of the low transformation efficiency during transformation. Despite the high numbers of embryos exposed to Agrobacterium cells, only a single transformed plant was found after regeneration. The recovery of this plant suggests that the vector is functional, and capable of transferring large DNA cargo, however at a low rate. There are many reasons that the 
transformation efficiency may be so low. First, the rate of transformation with any telomere truncation vector will be lower than that of a normal vector without telomere. There are several reasons for this reduction. First, the presence of telomere repeats delays the growth of bacterial cells, including Agrobacterium. This delayed growth rate implies a reduced capacity to infect embryos during the original transformation. Secondly, truncation will often result in the loss of necessary genetic material. Truncations of A chromosomes are more likely as they are the predominant genetic material in the cell, even when several B chromosomes are present. As a result, many of these truncation events will be selected against during the transformation process. Additionally, as the insertion of telomere is random, there is a $50 \%$ chance that truncation events will result in the loss of the inserted transgene itself. The shortened B chromosome in figure 39 , may be the result of such an event, though it is not possible to examine if this is the case.

It is worth noting that the single-gene FISH probe in figure 40 displayed signal at the tip of the long arm of chromosome 1 . This suggests that the transgene was either inserted at the tip of the chromosome arm, or that the chromosome was truncated upon transgene insertion. Examination of the regenerated plant shows white sectors throughout the leaves and stalk (figure 37). TAIL PCR experiments to determine the transgene insertion location with primers extending from the left border showed that the transgene could be found in a retrotransposon. As these elements are present throughout the genome, it is difficult to determine the exact insert location. Additionally, attempts to perform 
TAIL PCR through the telomere sequences at the right border were unsuccessful. This may be the result of the difficulty of polymerase extension through telomere repeats, or could also be due to a truncation event. As the plant did not survive past a couple of weeks in soil, additional experimentation is not possible.

The transformation efficiency was also lower as the transfer of high molecular weight DNA will reduce the overall transformation efficiency. This effect was previously demonstrated in tomato transformation, where empty vector, $30 \mathrm{~kb}$, and $150 \mathrm{~kb}$ T-DNA vectors were examined for transformation efficiency (Frary and Hamilton, 2001). The study showed that not only was there a reduction in transformation efficiency with BiBAC vectors, but additionally that transformation efficiency was lowered with larger T-DNA inserts. As the total size of the T-DNA inserts attempted in this study were over 67 and $110 \mathrm{~kb}$, the reduction in transformation efficiency may be partly attributed to the large size of the T-DNA insert. When also combined with the presence of telomere repeats, which will further reduce the transformation efficiency as previously discussed, the low transformation efficiency found should be of little surprise.

Through experimentation, it may be possible to increase the transformation efficiency by varying the aspects of the transformation process. For instance, due to the large size and inclusion of telomere sequences, the Agrobacterium cells could be observed growing much slower than typical Agrobacterium cultures containing standard cloning vectors. As a result, extending the infection time may lead to an increase in transformed cells. 
Additionally, altering the Agrobacterium delivery method or altering the growth media may increase transformation rate as well. Due to constraints of time and materials, these factors were only slightly varied in the current work.

The ability to produce minichromosomes through BiBAC vectors remains unknown following this work. While the vector was demonstrated to contain a large amount of telomere repeats, and was able to transfer a large cargo to maize cells, it was not shown to produce a verifiable minichromosome. However, as the number of plants regenerated with the vector was only one, additional work may demonstrate that the vector is capable of producing a minichromosome.

\section{References}

Frary, A., and Hamilton, C.M. (2001). Efficiency and stability of high molecular weight DNA transformation: an analysis in tomato. Transgenic Research $10,121-132$.

Gaeta, R.T., Masonbrink, R.E., Zhao, C., Sanyal, A., Krishnaswamy, L., and Birchler, J.A. (2013). In vivo modification of a maize engineered minichromosome. Chromosoma 122, 221-232.

Gao, Z., Fu, S., Dong, Q., Han, F., and Birchler, J.A. (2011). Inactivation of a centromere during the formation of a translocation in maize. Chromosome Research 19, 755-761.

Gelvin, S.B. (2010). Plant proteins involved in Agrobacterium-mediated genetic transformation. Annual Review of Phytopathology 48, 45-68.

Hamilton, C.M., Frary, a, Lewis, C., and Tanksley, S.D. (1996). Stable transfer of 
intact high molecular weight DNA into plant chromosomes. Proceedings of the National Academy of Sciences of the United States of America 93, 9975-9979.

Han, F., Lamb, J.C., and Birchler, J.A. (2006). High frequency of centromere inactivation resulting in stable dicentric chromosomes of maize. Proceedings of the National Academy of Sciences 103, 3238-3243.

Han, F., Gao, Z., and Birchler, J. a (2009). Reactivation of an inactive centromere reveals epigenetic and structural components for centromere specification in maize. The Plant Cell 21, 1929-1939.

He, R., Pan, J., Zhu, L., and He, G. (2010). Agrobacterium-Mediated Transformation of Large DNA Fragments Using a BIBAC Vector System in Rice. Plant Molecular Biology Reporter 28, 613-619.

Jones, N., and Houben, A. (2003). B chromosomes in plants: escapees from the A chromosome genome? Trends in Plant Science 8, 417-423.

McCaw, M., Swyers, N., Graham, N., Cody, J., Zhao, C., and Birchler, J. (2016a). Preparation of Chromosomes from Zea mays. Current Protocols in Plant Biology 1, 501-509.

McCaw, M., Graham, N., Cody, J., Swyers, N., Zhao, C., and Birchler, J. (2016b). Fluorescence In Situ Hybridization to Maize (Zea mays) Chromosomes. Current Protocols in Plant Biology 1, 530-545.

Murray, A., and Szostak, J. (1983). Construction of artificial chromosomes in yeast. Nature 305, 189-193.

Phan, B.H., Jin, W., Topp, C.N., Zhong, C.X., Jiang, J., Dawe, R.K., and Parrott, 
W. a (2007). Transformation of rice with long DNA-segments consisting of random genomic DNA or centromere-specific DNA. Transgenic Research $16,341-351$.

Robinson, J., Thorvaldsdottir, H., Winckler, W., Guttman, M., Lander, E., Getz, G., and Mesirov, J. (2011). Integrative Genomics Viewer. Nature Biotechnology 29, 24-26.

Shizuya, H., Birren, B., Kim, U.J., Mancino, V., Slepak, T., Tachiiri, Y., and Simon, M. (1992). Cloning and stable maintenance of 300-kilobase-pair fragments of human DNA in Escherichia coli using an F-factor-based vector. Proceedings of the National Academy of Sciences of the United States of America 89, 8794-8797.

Singer, T., and Burke, E. (2003). High-throughput TAIL-PCR as a tool to identify DNA flanking insertions. Methods in Molecular Biology (Clifton, N.J.) 236, $241-272$.

Vega, J.M., Yu, W., Han, F., Kato, A., Peters, E.M., Zhang, Z.J., and Birchler, J. a. (2008a). Agrobacterium-mediated transformation of maize (Zea mays) with Cre-lox site specific recombination cassettes in BIBAC vectors. Plant Molecular Biology 66, 587-598.

Vega, J.M., Yu, W., Kennon, A.R., Chen, X., and Zhang, Z.J. (2008b). Improvement of Agrobacterium-mediated transformation in $\mathrm{Hi}-\mathrm{Il}$ maize (Zea mays) using standard binary vectors. Plant Cell Reports 27, 297305.

Yu, W., Lamb, J.C., Han, F., and Birchler, J. a (2006). Telomere-mediated 
chromosomal truncation in maize. Proceedings of the National Academy of Sciences 103, 17331-17336.

Yu, W., Han, F., Gao, Z., Vega, J.M., and Birchler, J. a (2007). Construction and behavior of engineered minichromosomes in maize. Proceedings of the National Academy of Sciences of the United States of America 104, 89248929. 


\section{Chapter 6: Conclusion}

Biotechnology is a powerful tool to help combat the issue of food security. The advent of molecular techniques that can be applied with traditional breeding methods, allows researchers to tackle specific problems while also continuing to increase yields. Additionally, in the lab, use of biotechnology has allowed researchers to address specific biological questions through both forward and reverse genetic approaches. Despite the broad capabilities and power of the technology, the techniques have remained largely unchanged in maize since their inception in the early 1990s. The work presented herein seeks to improve upon the established transgenic technologies already developed for maize.

Chapter one examined the improvements that industry researchers have made to the transformation protocols and media. By examining the claims made in industry patents, a consensus protocol was made that comprised the most common alterations to the standard academic protocol. These changes mostly involved manipulations to the media composition, which allowed industry researchers to greatly accelerate the transformation process. By including growth hormones in the various media formulations, the growing callus cells could be forced into embryoid development much more quickly than standard protocols. Tests utilizing Hi-Il embryos with the new consensus protocol found that embryos did indeed develop much more quickly than with standard protocols. Using hormones in early media allowed even recalcitrant line B73 to be regenerated. 
Chapter two examined the activity of two recombinases, ФC31 integrase and excisionase. These recombinase proteins, which have not been previously demonstrated in maize cells but are used frequently in animal systems, allow for unidirectional recombination reactions. The use of a unidirectional recombinase system would be a great improvement over the commonly used Cre/loxP system, which is bidirectional and reversible when used for site-specific genomic insertion. Using the GUS reporter system, both $\Phi C 31$ integrase and excisionase were demonstrated to be functional when integrated into the genome and when expressed transiently.

Chapter three examined whether ФС 31 integrase could be used for sitespecific integration. A target recombination site was inserted into the genome that allowed for removal of the bialaphos selection cassette while allowing for continued use of the inserted ubiquitin promoter. Three different insertions of this target site were found in the maize genome, that were then demonstrated to be capable of bialaphos selection removal. Target insertion was demonstrated by inserting a promoterless GUS reporter construct. Upon integration into a target site, the GUS reporter would be placed behind the already present ubiquitin promoter and thus making the GUS reporter protein. This reporter system was demonstrated both transiently and with an integrated $\Phi C 31$ integrase with the GUS reporter. While the insertion could not be molecularly verified with this strategy, the proof-of-concept experiment demonstrates that further work utilizing a selection gene and regeneration should be performed. 
Chapter four is a published protocol on the production of minichromosomes in maize in the journal Methods in Molecular Biology. The protocol demonstrates the cloning strategy for the insertion of telomere sequences into cloning vectors that is best utilized in producing telomeretruncation vectors. Telomere sequences are repetitive in nature, which makes them difficult to work with for cloning purposes. By excising telomere sequences from agarose gels and performing the cloning reaction within the gel matrix, many of the difficulties in performing the cloning reaction can be alleviated. Additionally, the protocol demonstrates an efficient method for screening for telomere insert presence and size through Southern hybridization utilizing a radiolabeled oligo.

Chapter five examines the use of binary bacterial artificial chromosomes (BiBAC) for the production of minichromosomes. Traditionally, minichromosomes are produced through the use of standard cloning vectors, which can not generally be constructed at sizes larger than $20 \mathrm{~kb}$. This size limit prevents much more than a single gene from being transferred during minichromosome production. This size limit restricts large genes and gene clusters from being introduced during truncation. As a result, additional genetic cargo would need to be inserted at a later date through a site-specific insertion strategy. By employing a BiBAC vector during minichromosome production, up to $300 \mathrm{~kb}$ of genetic cargo could be inserted at one time, expanding the utility of minichromosome technology. The current work constructed a BiBAC vector that contained a removable selection cassette and recombination site for future insertion by $\Phi$ C31 
integrase, which was demonstrated to function in maize as described above. Additionally, a multiple cloning site consisting of rare-base cutting enzymes was inserted into the vector to allow for cloning of large genetic fragments. As a proof of concept, large fragments of yeast genomic DNA were inserted into the vector. The vector was then used for maize transformation into immature Hi-II embryos. After rigorous transformation experiments only a single regenerated plant was produced. While the plant contains a shortened B chromosome, it is not possible to determine if the chromosome resulted from a truncation. While the production of a minichromosome can not be determined by these experiments, the vector itself was demonstrated to be capable of transferring large genetic cargos to maize cells. Additionally, as previous experiments have demonstrated minichromosome production with similar telomere arrangements, it may be possible to produce a minichromosome if more regenerated plants can be produced.

Taken together, the work described here makes progress towards the goal of a more efficient transformation system for maize. By combining site-specific insertion with improved regeneration media, it may be possible to greatly improve the speed at which maize can be transformed. Additionally, by combining sitespecific transformation with BiBAC minichromosomes, it should be possible to produce minichromosomes with large cargo initially. This would allow for a large array of genetic elements to be contained on a single minichromosome, easing the burden of genetic manipulation in the future. 


\section{VITA}

Nathaniel Graham was born in St. Louis Missouri to his parents, Carol and Mark Graham. He completed high school at Francis Howell High School in 2007 before starting college at Truman State University. During his time at Truman State he was an intern at Monsanto Company, where he gained an appreciation for agricultural biotechnology. After obtaining a bachelor of science degree at Truman State, he moved to graduate school at the University of Missouri in the lab of James Birchler. 